\title{
Auf dem Wege zu Oligocyclopropanen über Transformationen an 1,6-substituierten trans-Endiinen
}

\author{
Dissertation \\ zur Erlangung des Doktorgrades \\ der Mathematisch-Naturwissenschaftlichen Fakultäten \\ der Georg-August-Universität zu Göttingen
}

vorgelegt von

Matthias Wrobel

aus Verden

Göttingen 1999 
D 7

Referent: $\quad$ Prof. Dr. A. de Meijere

Korreferent: $\quad$ Prof. Dr. A. Zeeck

Tag der mündlichen Prüfung: 02.11.1999 
Die vorliegende Arbeit wurde in der Zeit vom Juni 1996 bis August 1999 im Institut für Organische Chemie der Universität Göttingen angefertigt.

An dieser Stelle möchte ich mich bei meinem Lehrer Herrn Prof. Dr. A. de Meijere für die interessante Themenstellung und die damit verbundene Unterstützung während meiner Promotionszeit bedanken. 
$\begin{array}{ll}\text { B. Hauptteil } & 10\end{array}$

1. Darstellung von trans-1-Ethinyl-2-hydroxymethylcyclopropanen 10

1.1. Darstellung von trans-1-Ethinyl-2-benzyloxymethylcyclopropan 10

1.1.1. Vorüberlegungen 10

1.1.2. Einseitige Reduktion und Schützung von trans-1,2-Cyclopropandicarbonsäureester 12

1.1.3. Reduktion und anschließende Swern-Oxidation der zweiten Esterfunktion am Cyclo$\begin{array}{ll}\text { propanring } & 14\end{array}$

1.1.4. Umsetzungen zum trans-1-Ethinyl-2-benzyloxymethylcyclopropan 16

1.2. Versuch zur Darstellung von 1,2-Diethinylcyclopropan 18

1.3. Cyclopropanierungen an Pent-2-en-4-in-1-olen 19

$\begin{array}{ll}\text { 1.3.1. Vorüberlegungen } & 19\end{array}$

1.3.2. Darstellung und Schützung von Pent-2-en-4-in-1-ol 20

1.3.3. Cyclopropanierungen 21

2. Kupplungsreaktionen und Cyclopropanierungen an Kupplungsprodukten 26

2.1. Vorüberlegungen 26

2.2. Palladium-katalysierte Kupplung von Acetylenen zu Endiinen 29

2.3. Darstellung von Chloreninen aus Acetylenen und trans-1,2-Dichlorethen 31

2.4. Palladium-katalysierte Kupplung von Chloreninen mit Acetylenen 33

2.5. Cyclopropanierungen an endständigen Allylalkohol-Funktionen ungesättigter Vorläufer

3. 1,2-Dihydroxylierungen an Endiinen 36

3.1. Vorüberlegungen 36

3.2. Dihydroxylierungen nach Sharpless 37

3.3. Dihydroxylierungen mit Rutheniumtetroxid 41

3.4. Andere Darstellungversuche für Diindiole 44

4. Folgereaktionen der Dihydroxylierungsprodukte 48

$\begin{array}{lll}\text { 4.1. } & \text { Vorüberlegungen } & 48\end{array}$

4.2. Reduktion der Dreifachbindungen von Diindiolen 49

4.3. Doppelte Cyclopropanierung und Corey-Winter-Eliminierung zu 1,2-Bis(bicyclopropyl)ethen 
C. Experimenteller Teil 54

1. Allgemeines $\quad 54$

2. Darstellung der Verbindungen $\quad 56$

2.1. Verbindungen ausgehend vom Cyclopropran-1,2-dicarbonsäureethylmethylester 56

2.2. Verbindungen ausgehend vom Pent-2-en-4-in-1-ol 61

2.3. Kupplungsreaktionen 66

2.3.1. Allgemeine Arbeitsvorschriften für Kupplungsreaktionen 66

2.3.2. Enine 66

2.3.3. Endiine $\quad 69$

2.3.4. Trien- und Diendiine 72

2.4. Cyclopropanierungen an mehrfach ungesättigten Allylalkoholen 73

2.5. Oxidationen an Endiinen 76

2.5.1. Dihydroxylierungen nach Sharpless 76

2.5.2. Dihydroxylierungen mit Rutheniumtetroxid 78

2.5.3. Dihydroxylierungen mit Kaliumpermanganat 79

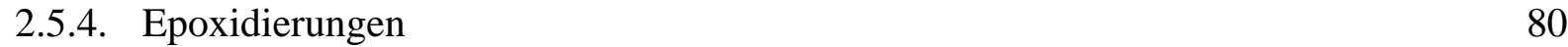

2.6. Transformationen an dihydroxylierten Endiinen $\quad 82$

D. Zusammenfassung $\quad 84$

$\begin{array}{lll}\text { E. Literatur } & 86\end{array}$

$\begin{array}{ll}\text { F. Spektren } & 91\end{array}$ 


\section{A. Einleitung}

Die Natur überrascht uns immer wieder mit dem Auftreten von Lebewesen ungewöhnlichen Phänotyps. Diese natürliche Vielfalt und Variationsbreite setzt sich auf molekularer Ebene fort. In Pflanzen und Lebewesen entdeckt man häufig Moleküle, die man in dieser Form niemals erwartet hätte. Eines dieser außergewöhnlichen Strukturelemente ist der Cyclopropanring, der in vielen Naturstoffen anzutreffen ist ${ }^{[1]}$. Schon lange ist die Chrysanthemumsäure $1^{[2]}$ bekannt, die aus den getrockneten Blütenköpfen verschiedener Chrysanthemum-Arten isoliert werden kann. Durch einfaches Pulverisieren oder Extrahieren wird ein Pyrethrum genanntes Pulver gewonnen, welches seit langem als wirksames Insektizid eingesetzt wird. Die Hauptwirkstoffe dieses Mittels sind die sechs optisch aktiven Ester der (+)-trans-Chrysanthemumsäure und der (+)-trans-Pyrethrinsäure, die ebenfalls einen Cyclopropanring enthältt ${ }^{[3]}$.

Häufig finden sich Cyclopropanringe auch in Fettsäuren, wie beispielsweise der Mycolsäu$\mathrm{re}^{[4]}$. Sie ist ein Hauptbestandteil der Zellwand des Mycobacterium tuberculosis und trägt in ihrer Seitenkette zwei Cyclopropanringe. Alle diese Verbindungen, die einzelne Cyclopropanringe enthalten, sind schon länger bekannt.

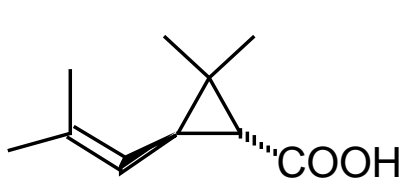

1

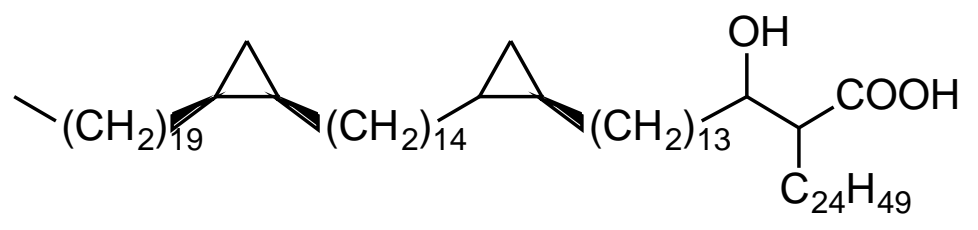

2

Auf der Suche nach neuen Wirkstoffen bedient man sich häufig natürlicher Vorläufer. Bei solchen Screenings entdeckte man die beiden ungewöhnlichen Substanzen FR-900848 $3^{[5]}$ und U-106305 4[6]. FR-900848 wurde aus dem Stamm Streptoverticillium fervens isoliert und wies eine hochspezifische Wirkung gegen filamentöse Pilze auf. Die Seitenkette von 3 enthält fünf Cyclopropylgruppen, von denen vier direkt miteinander verknüpft sind. U106305 fand man bei einem Screening auf Inhibitoren des Cholesterylestertransferproteins 
(CETP). Die Seitenkette von 4 enthält sogar sechs Cyclopropanringe, von denen fünf zu einer Pentacyclopropyleinheit verknüpft sind.

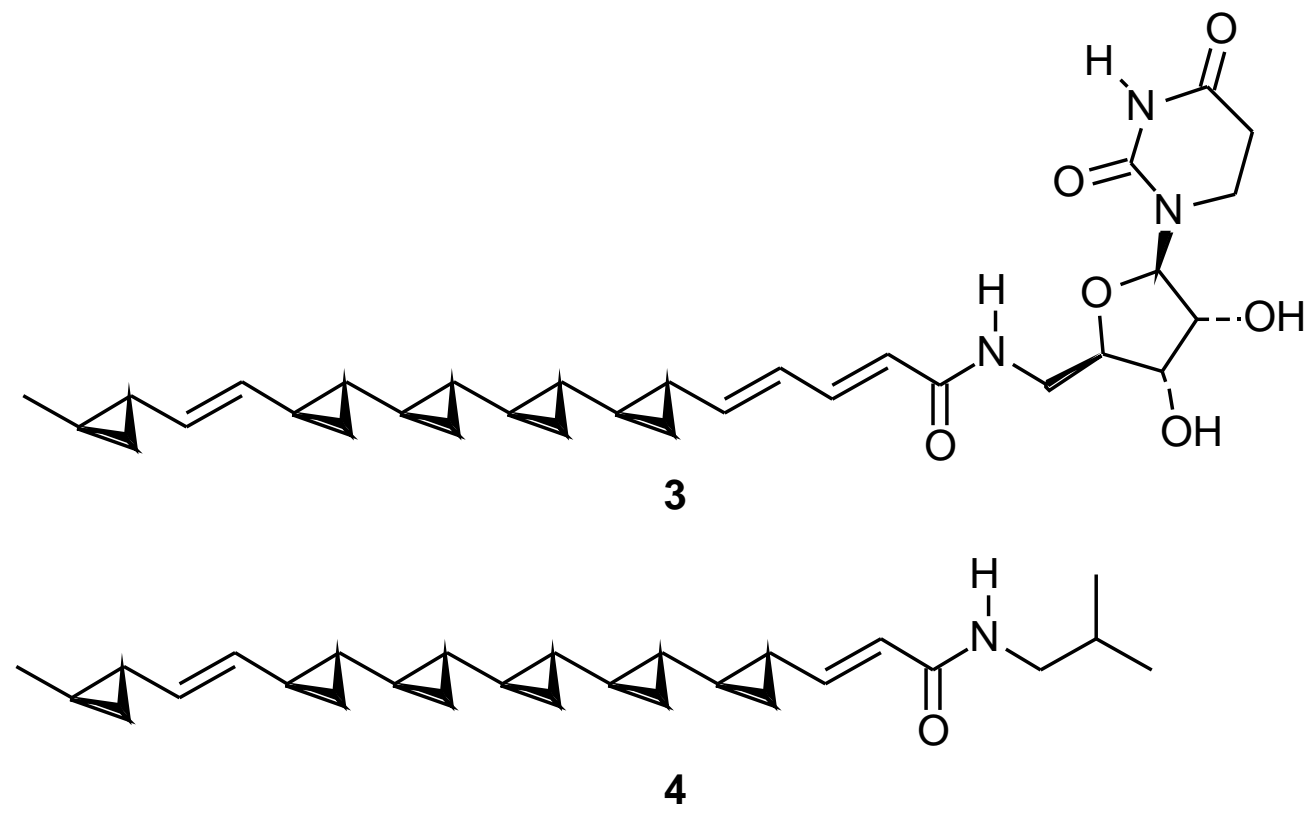

Bis zur Entdeckung von 3 und 4 war wenig über die Synthese von Cyclopropylketten bekannt, weiterhin war die Stereochemie der Seitenketten nicht aufgeklärt. In dem Bestreben, diese ungewöhnlichen Naturstoffe mit den Methoden der organischen Synthese darzustellen, entwickelten mehrere Arbeitskreise unterschiedliche Synthesestrategien ${ }^{[7]}$.

Die Totalsynthesen von $3^{[8]}$ und $\mathbf{4}^{[9]}$ gelangen zuerst dem Arbeitskreis von Barrett. Über eine Folge von doppelter Cyclopropanierung an 5, Oxidation, Wittig-Reaktion, Reduktion und anschließender doppelter Cyclopropanierung an 6 konnte sowohl die tetra- und nach dem gleichen Schema auch die Pentayclopropylkette in relativ wenigen Schritten aufgebaut werden. Durch Vergleiche mit den Naturstoffen klärte man auch die absoluten Konfigurationen der Seitenketten auf.

Wie schon vorher vermutet, stehen alle Cyclopropanringe trans zueinander. Anhand von Röntgenstrukturen an Tetra- und Pentacyclopropyleinheiten erkennt man, daß sich die Oligocyclopropylketten helical anordnen. 
<smiles>OC/C=C/C=C/CO</smiles>

5

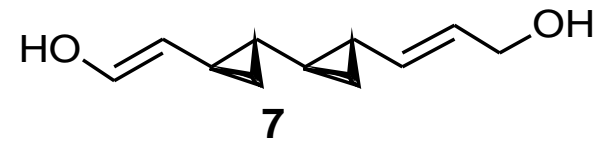

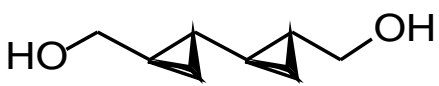

6
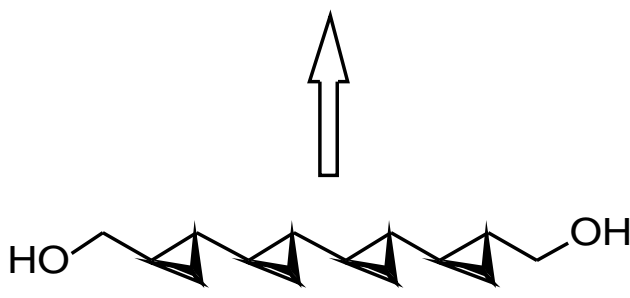

8

Schema 1. Synthese der Oligocyclopropane nach Barrett et al.

Eine wertvolle Hilfe bei der Synthese war die Cyclopropanierung nach Charette ${ }^{[10]}$, die unter Verwendung eines chiralen Boronsäureesters die höchste Diastereoselektivität bei der Cyclopropanierung von Allylalkoholen 9 erreicht.<smiles>[R]C([R])=C([R])CO</smiles>

9

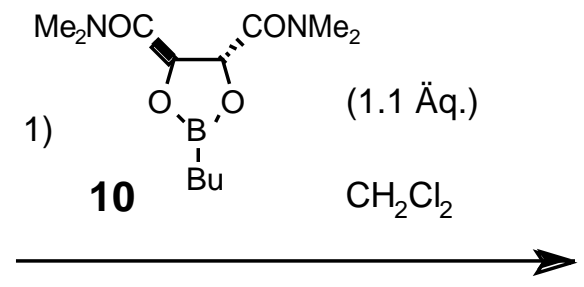

2) $\mathrm{Zn}\left(\mathrm{CH}_{2}\right)_{2}(2.2$ Äquiv.) $\mathrm{CH}_{2} \mathrm{Cl}_{2}, 25^{\circ} \mathrm{C}, 2 \mathrm{~h}$<smiles>[R]C1([R])CC1([R3])CO</smiles>

11

Ausbeuten $\geq 80 \%$ 91-94\%ee

Während sich die meisten Synthesen von Oligocyclopropylen auf die diastereoselektive Cyclopropanierung von Allylalkoholen stützen, wurde von von Seebach ${ }^{[11]}$ ein gänzlich anderer Weg zur Synthese von unsubstituierten Quatercyclopropylen entwickelt. Schlüsselreaktion dafür ist die Dimerisierung von Bicyclopropyliden, das in guten Ausbeuten in einer dreistufigen Synthese darstellbar ist ${ }^{[12]}$. Das Diastereomer meso-13 konnte durch Tieftemperatur- 
kristallisation in reiner Form erhalten werden, und je nach Reduktionsmethode bilden sich unterschiedliche Diastereomere des Quatercyclopropyls 14 (Schema 1).

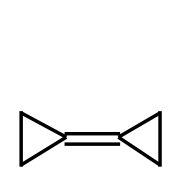

12

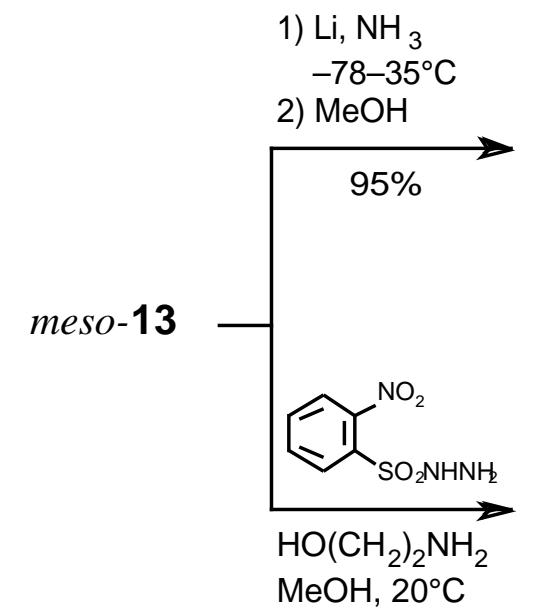

1) BuLi

2) $\left[\mathrm{CulBu}_{3} \mathrm{P}\right]_{4}$

3) $\mathrm{O}_{2}$

$80 \%$

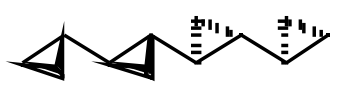

$\left(R^{\star} S^{\star} R^{\star} S^{\star}\right)-14$

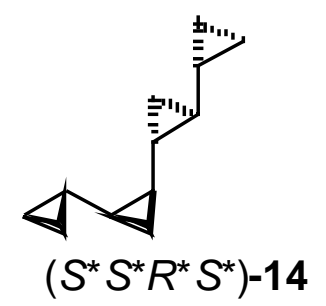

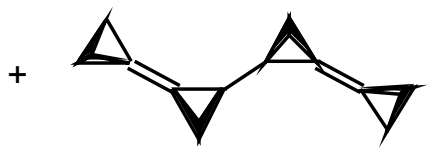

$d, l-13$

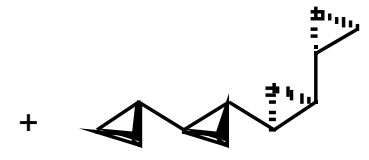

$\left(R^{\star} S^{\star} R^{\star} R^{\star}\right)-14$

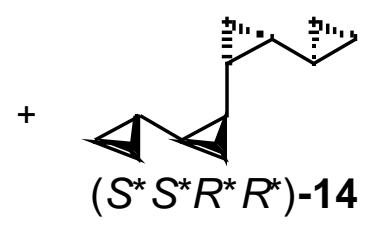

Schema 2. Quatercyclopropylen aus Bicyclopropyliden.

So erfolgreich auch die Synthese von Oligocyclopropylketten durchgeführt wurde, StrukturWirkungs-Beziehungen dieser Verbindungsklasse im Hinblick auf die Wirkweise von $\mathbf{3}$ und 4 sind bisher nicht veröffentlicht. Ebenso ist der biochemische Abbau der cyclopropanierten Fettsäuren noch nicht genauer untersucht. Aus diesem Grund synthetisierte S. Löhr einfache Bicyclopropylfettsäuren mit gerad- und ungeradzahligen Seitenketten ${ }^{[13]}$.

In ersten Fütterungsexperimenten wurden jedoch noch keine Organismen gefunden, die zum Abbau der Cyclopropanringe fähig waren. Es wurde nur Bicyclopropylcarbonsäure bei ungeradzahligen und Bicyclopropylessigsäure bei geradzahligen Seitenketten an der Bicyclopropylgruppe der Ausgangssubstanzen isoliert. Daraus läßt sich schließen, daß der Abbau ausschließlich nach dem bekannten Mechanismus der $\beta$-Oxidation stattgefunden hat ${ }^{[14]}$. 3 und $\mathbf{4}$ weisen jeweils eine $\mathrm{C}_{18}$-Seitenkette auf, die sich durch einen zusätzlichen Cyclopropanring in $\mathbf{4}$ unterscheidet, an dessen Stelle sich bei $\mathbf{3}$ eine Doppelbindung befindet. Vermut- 


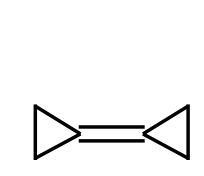

12
1) $n \mathrm{BuLi}, \mathrm{THF}$

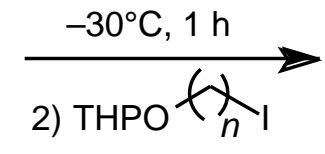

$-78-20^{\circ} \mathrm{C}$

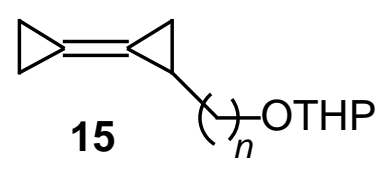

1) $\mathrm{Li}$ (3 Äq.), $\mathrm{NH}_{3}$ $-78^{\circ} \mathrm{C}, 2 \mathrm{~h}$

2) $\mathrm{MeOH}$, $-78^{\circ} \mathrm{C}-20^{\circ} \mathrm{C}$

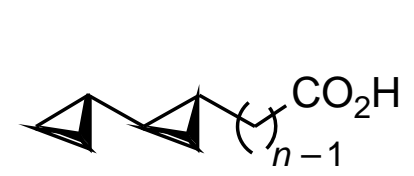

17

\section{$\mathrm{n}=\mathbf{2}-\mathbf{8}$}

1) $\mathrm{H}^{+}, \mathrm{MeOH}$

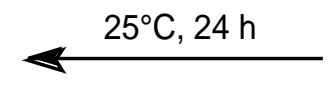

2) Jones-Reagenz

Aceton, $0^{\circ} \mathrm{C}$

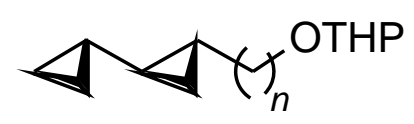

16

Schema 3. Bicyclopropylfettsäuren aus Bicyclopropyliden.

lich liegt deshalb die Wirkstruktur in der Seitenkette. Um genauere Untersuchungen zu Struktur und Wirkung durchführen zu können, braucht man einen einfachen synthetischen Zugang zu Ketten, die eine unterschiedliche Abfolge von Cyclopropanringen aufweisen. Die Synthese sollte zudem noch so flexibel sein, neue Gruppen beispielsweise mit Heteroatomen einführen zu können.

Die Synthesestrategie, die in dieser Arbeit verfolgt wurde, geht von endständig substituierten trans-Endiinen 19 aus, die durch mehrstufige Umsetzung zu Tricyclopropylen 21 umgesetzt werden sollten. Die Endiine sollten durch einstufige doppelte Kupplung oder zweistufige Kreuzkupplung von 1,2-Dihalogenalkenen mit geeigneten Alkinen 18 aufgebaut werden (Schema 4).

Verwendet man als Kupplungspartner geschützte 2-Ethinyl-1-hydroxymethylcyclopropane 24 und andere kurzkettige geschützte Alkohole mit endständiger Alkinfunktion, würden sich gut Cyclopropylketten mit drei bis fünf Ringen aufbauen lassen. Nach dem Entschützen der endständigen Alkoholfunktionen in 21 wären weitere Syntheseschritte möglich. 
<smiles>[R]C#C</smiles><smiles>[R]</smiles><smiles>C#CC</smiles>

18

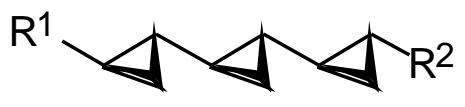

21

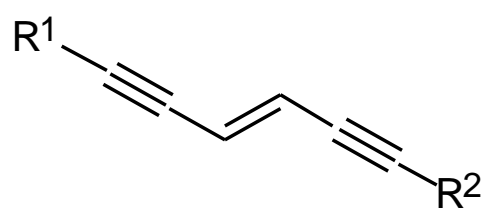

19
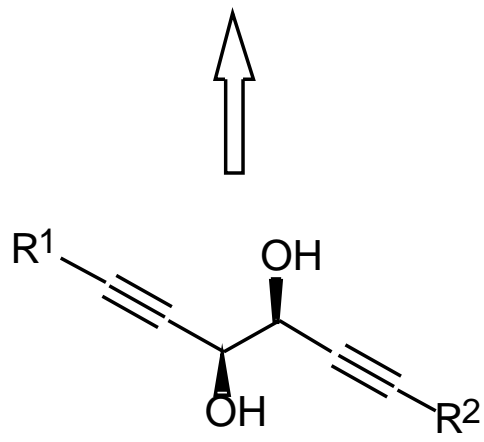

20

Schema 5. Von Endiinen zu Tricyclopropylen.

Die Synthese von geschützem trans-1-Hydroxymethyl-2-ethinylcyclopropan (24) ausgehend von trans-1,2-Cyclopropandicarbonsäureester (22) wurde in der Diplomarbeit begonnen und sollte nun verbessert und zu Ende geführt werden.
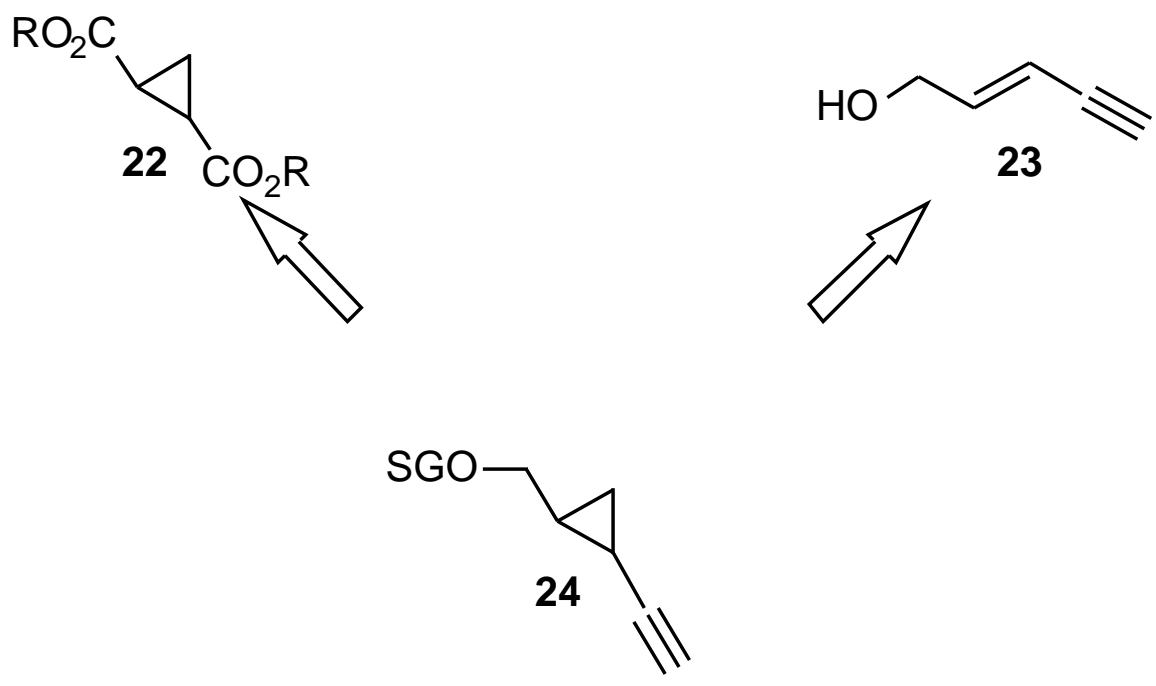

Schema 6. Darstellung geeigneter Cyclopropanderivate. 
Ein anderer Zugang zu 24 könnte über die Simmons-Smith-Cyclopropanierung ${ }^{[15]}$ von Pent2-en-4-in-1-ol (23) gelingen, das auch leicht über eine Cyclopropanierung nach Charette in enantiomerenreiner Form dargestellt werden könnte. Erste Versuche in der Diplomarbeit zeigten allerdings, daß Cyclopropanierungen an $\mathbf{2 3}$ unter den üblichen Reaktionsbedingungen nur sehr schlecht gelingen.

Schlüsselschritt zur Transformation der Endiine ist die Dihydroxylierung nach Sharpless ${ }^{[16]}$ (Schema 7). Über die Hydroxygruppe und das dadurch erzeugte Stereozentrum sollten die folgenden Umsetzungen an den benachbarten Dreifachbindungen gesteuert werden. In der Literatur findet man allerdings keine Beispiele derartiger Reaktionen an Endiinen. Schon während der Diplomarbeit hat sich herausgestellt, daß Endiine nur sehr schlecht oder gar nicht nach dem normalen Sharpless-Protokoll reagieren. Hier müßten andere Bedingungen oder Methoden zur Dihydroxylierung gefunden werden.

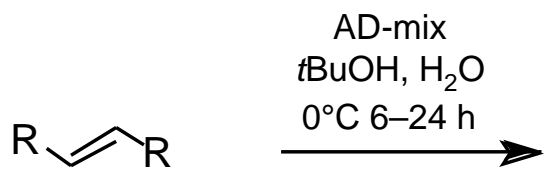

25

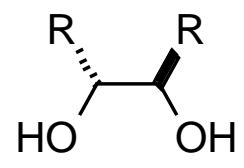

26

Schema 7. Dihydroxylierung nach Sharpless. 


\section{B. Hauptteil}

\section{Darstellung von trans-1-Ethinyl-2-hydroxymethylcyclopropanen}

\subsection{Darstellung von trans-1-Ethinyl-2-benzyloxymethylcyclopropan}

\subsubsection{Vorüberlegungen}

Ausgangsmaterialien für mehrstufige Synthesen sollten leicht und in großen Mengen darstellbar sein. Zur Synthese von trans-1-Ethinyl-2-benzyloxymethylcyclopropan (53) wurde aus diesen Gründen der 1,2-Cyclopropandicarbonsäureester (22) gewählt. Er ist einfach aus handelsüblichen Chemikalien im molaren Maßstab über bewährte Methoden herstellbar. Weiterhin besitzt er schon das Substitutionsmuster der Zielverbindung und läßt sich über bekannte Reaktionsschritte transformieren.

Über eine selektive halbseitige Reduktion würde aufgrund der Reaktionsbedingungen ausschließlich trans-2-Hydroxymethylcyclopropan-1-carbonsäureester (27) entstehen, der 
nach Schützung zu 28 über eine weitere Reduktion in den Alkohol 29 überführt werden kann.
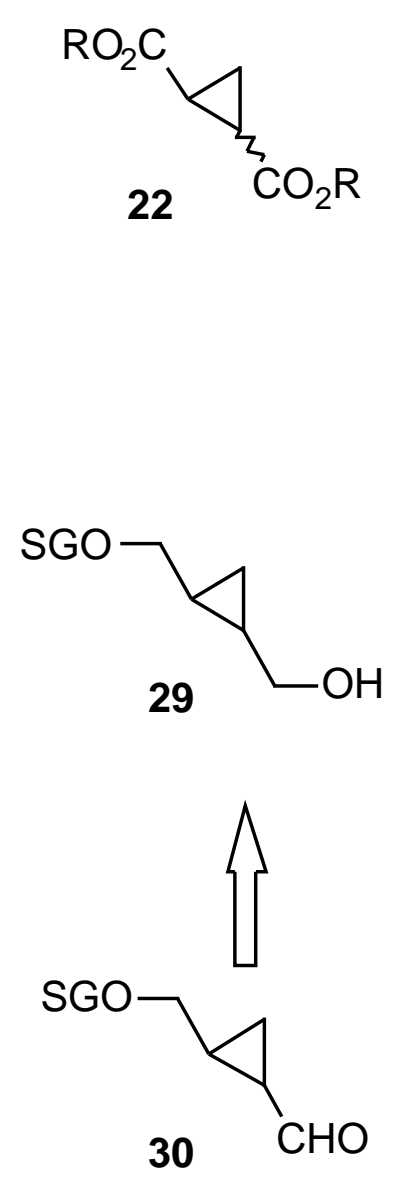
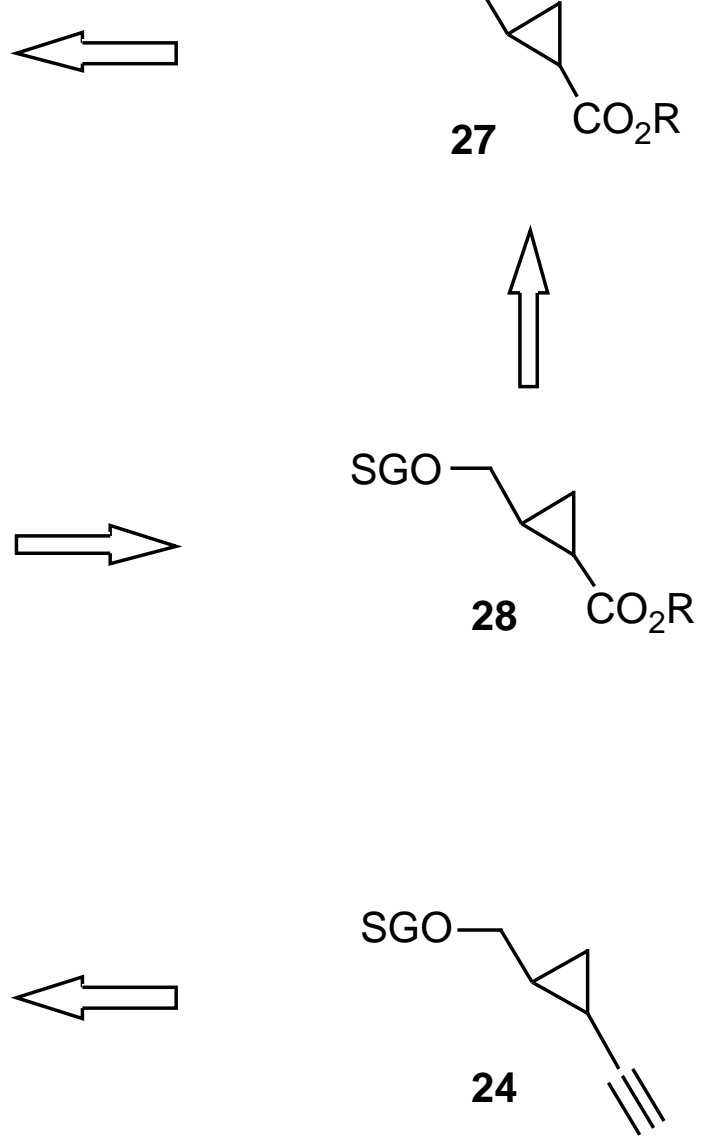

Schema 8. Synthese von geschützem trans-1-Hydroxymethyl-2-ethinylcyclopropan

Durch Oxidation von 29 erhält man den Aldehyd 30. Um die Sequenz um einen Reaktionsschritt zu verkürzen, wurde in der Diplomarbeit versucht, den Monoester 28 über eine kontrollierte Reduktion mit Diisobutylaluminiumhydrid in den Aldehyd $\mathbf{3 0}$ umzuwandeln. Dabei wurde beobachtet, daß diese Reaktion sehr stark von den Bedingungen und der Qualität des Reduktionsmittels abhing, deshalb ging man hier wieder zur zweistufigen Alternative über.

Die Umwandlung des Aldehyds $\mathbf{3 0}$ zum Alkin und Zielmolekül 24 wurde mit einer Variante der Wittig-Reaktion versucht und mit der Corey-Fuchs Reaktion erfolgreich durchgeführt. 


\subsubsection{Einseitige Reduktion und Schützung von trans-1,2-Cyclopropandicarbonsäureester}

Der 1,2-Cyclopropandicarbonsäureester (33) läßt sich leicht in einer Michael-Reaktion mit anschließendem Ringschluß von Chloressigsäureethylester (32) und Acrylsäuremethylester (XX) in 67\% erhalten. Die Synthese ist im molaren Maßstab durchführbar und ergibt ein 1: 1 Isomerengemisch von cis- und trans-33.<smiles>C=CC(=O)OC</smiles>

31<smiles>CCOC(=O)CCl</smiles>

32

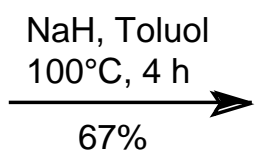

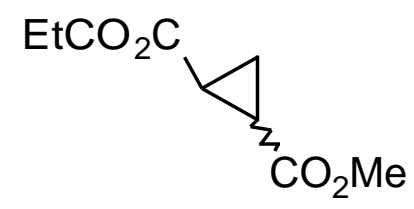

33

Die Isomere lassen sich zwar säulenchromatographisch trennen, was in diesem Fall nicht notwendig war, da unter den basischen Aufarbeitungsbedingungen der folgenden Reduktion eine Isomerisierung zu reinem trans-34 erfolgt.

Die einseitige Reduktion mit Lithiumaluminiumhydrid von 33 bei $-70{ }^{\circ} \mathrm{C}$ ergibt in $92 \%$ ein $1: 1$ Gemisch der beiden Ester 34-Et und 34-Me. Sie lassen sich weder destillativ noch säulenchromatographisch trennen. Da nach zwei Reaktionsschritten wieder ein einheitliches Produkt erhalten wird, mußten 34-Et und 34-Me nicht getrennt werden.

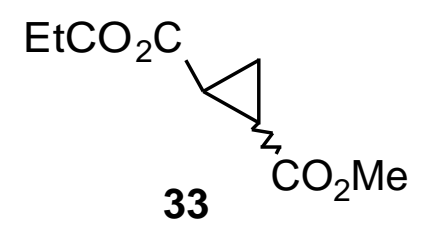

$\mathrm{LiAlH}_{4}$, THF

$-70^{\circ} \mathrm{C}, 3 \mathrm{~h}$
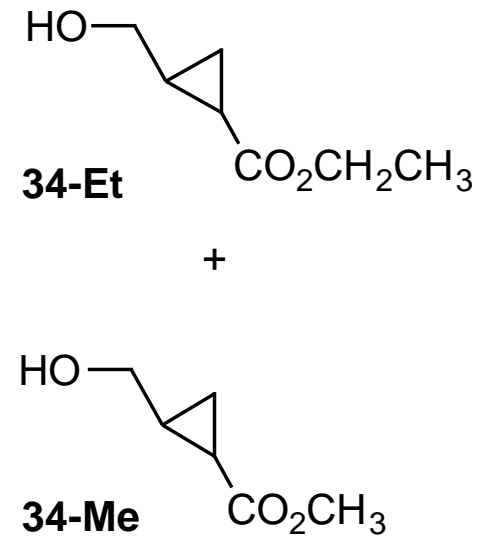

Im weiteren Syntheseverlauf wird eine säure- und basenstabile Schutzgruppe, die auch dem Angriff von Metallhydriden standhält und Oxidationen unbeschadet übersteht, benötigt. 
Dazu wurde die Benzylgruppe in 36-Et/Me mit einer Ausbeute von 91\% eingeführt.

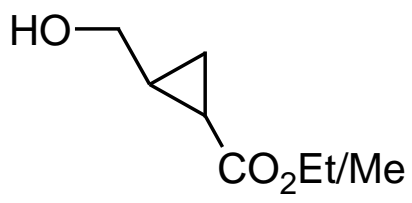

34-Et/Me
1) $\mathrm{NaH}, \mathrm{THF}$

2) $\mathrm{BnBr}, 20 \mathrm{~h}, \mathrm{RT}$

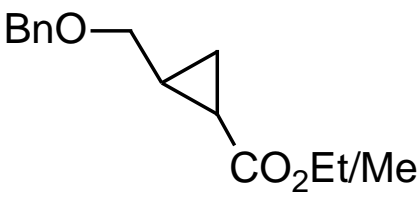

36-Et/Me

Eine weitere interessante Schutzgruppe für die Alkohol-Funktion in $\mathbf{3 4}$ wäre die tert-Butylgruppe. Normalerweise sind Alkylether als Schutzgruppen für Alkohole schlecht geeignet, da sie sich nur unter drastischen Bedingungen wieder spalten lassen. Die tert-Butylgruppe bildet hier eine Ausnahme, da sie ein relativ stabiles Kation bildet und sich so auch unter mäBig sauren Bedingungen wieder abspalten läßt. Um die tert-Butylgruppe einzuführen, addiert man Isobuten (37) unter Verwendung eines sauren Katalysators an den Alkohol. Die Einführung der tert-Butylgruppe in 34-Me gelang jedoch nicht. Das Edukt wurde unverändert wieder zurückgewonnen.

34-Me

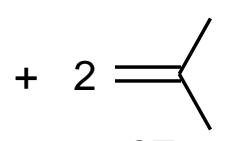

37
$\mathrm{HCl}, 20 \mathrm{~h}$

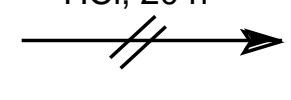

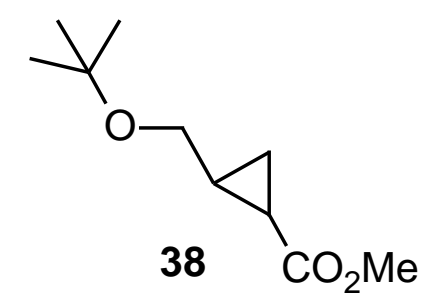


1.1.3. Reduktion und anschließende Swern-Oxidation der zweiten Esterfunktion am Cyclopropanring

Die Reduktion der zweiten Estergruppe an 36-Et/Me erfolgte ebenfalls mit Lithiumaluminiumhydrid und ergab in 91\% den Alkohol 39.

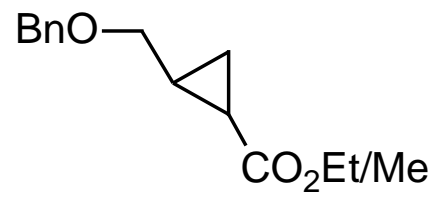

36-Et/Me
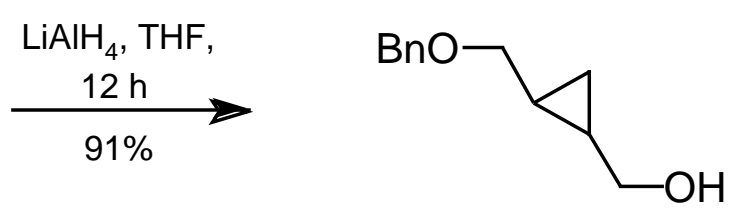

39

Es gibt mehrere Methoden Alkohole mit Dimethylsulfoxid (DMSO) in Gegenwart von Elektrophilen wie Dicyclohexylcarbodiimid, Acetanhydrid oder Oxalylchlorid zu Aldehyden zu oxidieren. Die gebräuchlichste ist die Swern-Oxidation, bei der man mit DMSO, Oxalylchlorid und Triethylamin arbeitet.

Das durch Oxalylchlorid aktivierte DMSO 41 reagiert mit dem Alkohol zu einem Sulfuran 43. 43 dissoziiert zum Sulfoniumsalz 44, das bei Zugabe von Triethylamin und Erwärmen 
zum Sulfonium-Ylid 45 umgesetzt wird. Über einen cyclischen Übergangszustand entsteht in einer $\beta$-Eliminierung der Aldehyd 47 und Dimethylsulfid (46).

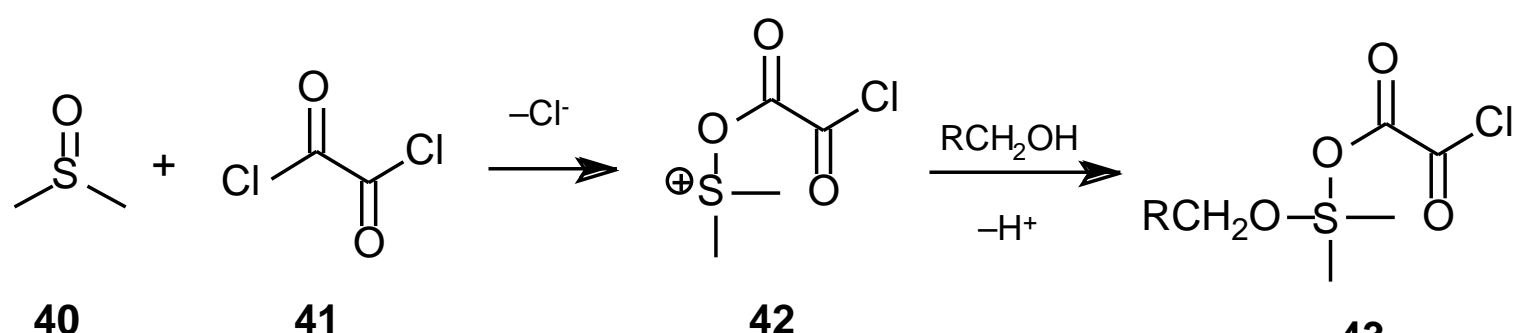

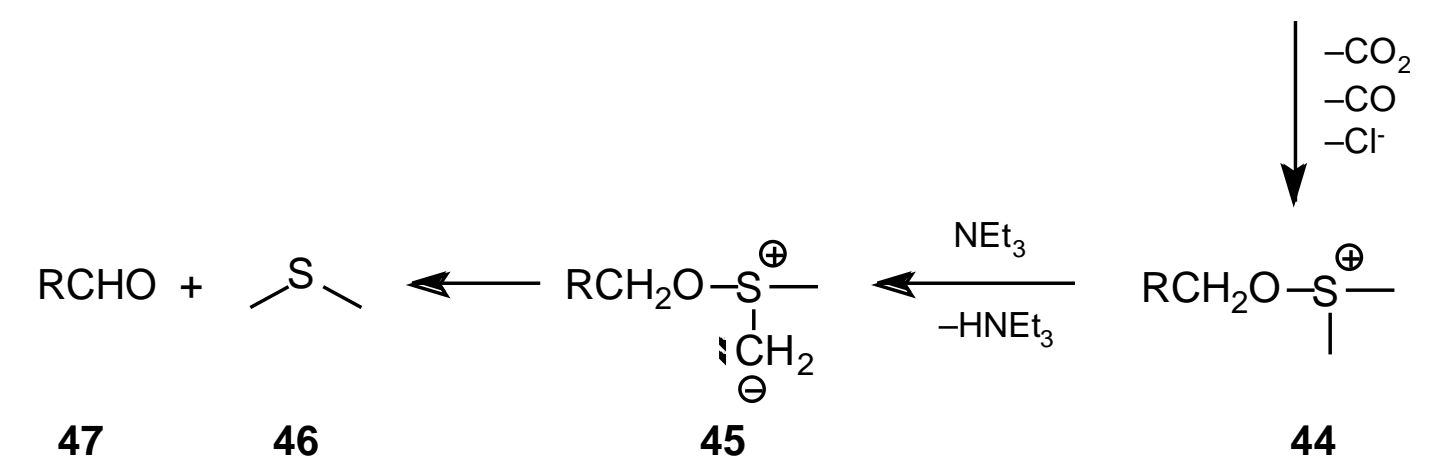

Schema 9. Mechanismus der Swern-Oxidation

Die Oxidation von 39 nach Swern erfolgte mit 85\% Ausbeute zum gewünschten Cyclopropylcarbaldehyd 48.

39

1) $\mathrm{DMSO},(\mathrm{COCl})_{2}, \mathrm{CH}_{2} \mathrm{Cl}_{2}$

$-70^{\circ} \mathrm{C}, 30 \mathrm{~min}$

2) $\mathrm{NEt}_{3}$

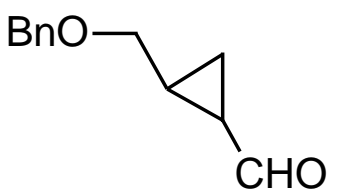

48 


\subsubsection{Umsetzungen zum trans-1-Ethinyl-2-benzyloxymethylcyclopropan}

Es wurden zwei Methoden angewandt, um die Aldehydgruppe am Cycloporpanring in eine Ethinylgruppe umzuwandeln, und zwar über eine Wittig-Reaktion mit (Brommethyl)triphenylphosphoniumbromid (49) und über die Corey-Fuchs-Reaktion.

Bei der angewandten Variante der Wittig-Reaktion ist es wichtig, daß Ylid $\mathbf{5 0}$ mit Kaliumtert-butanolat bei $-78{ }^{\circ} \mathrm{C}$ zu erzeugen. Arbeitet man mit $n$-Butyllithium, erhält man nicht reproduzierbare Ergebnisse, und bei höheren Temperaturen wird 50 vermutlich durch den entstehenden tert-Butylalkohol zerstört.

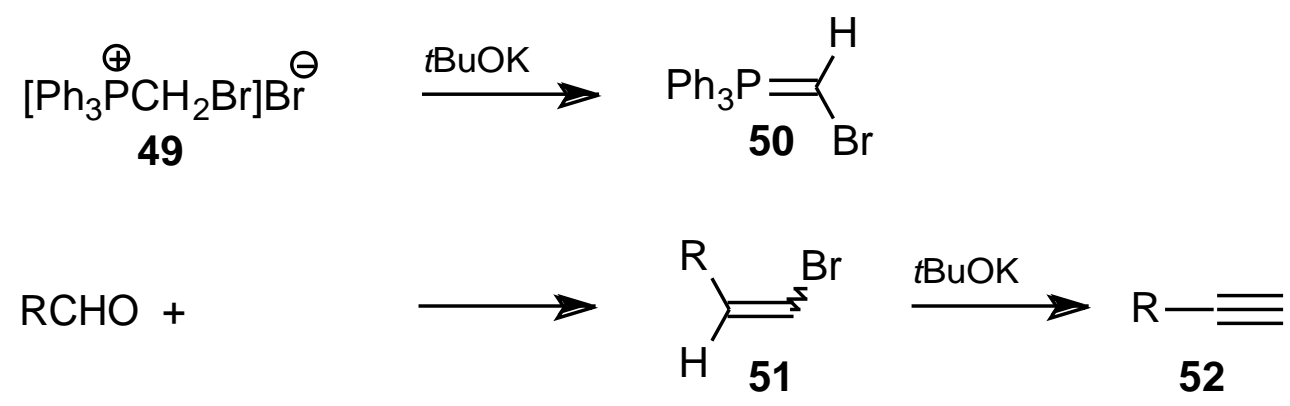

Schema 10.

Mit einem Äquivalent Base erhält man vorwiegend das (Z)-Bromalken 51, mit zwei Äquivalenten eliminiert $\mathbf{5 1}$ zum Alkin 52.

Bei der im Schema 10 vorgestellten Reaktion konnten bei der Umsetzung mit dem Aldehyd 48 nur Spuren des Vinylbromids gefunden werden, aber kein Produkt 53.

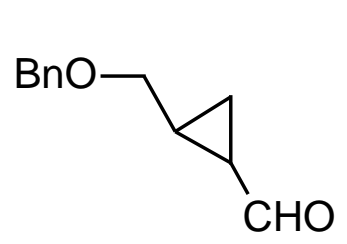

48

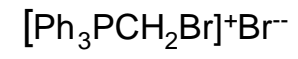

2 tBuOK, $-78^{\circ} \mathrm{C}$, THF

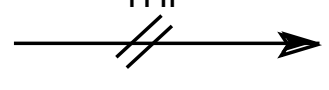

53

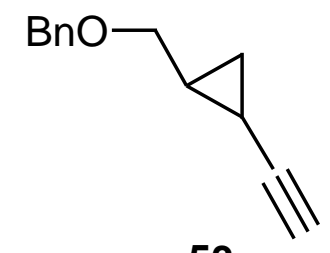

Als zweite Alternative zur Darstellung von 53 wurde die Corey-Fuchs-Reaktion durchgeführt. In deren erstem Schritt wird in situ aus Triphenylphosphan, Tetrabrommethan und Zink ein dibromsubstituiertes Phosphonium-Ylid gebildet, das dann mit dem Aldehyd zum 
1,1-Dibromalken $\mathbf{5 4}$ reagiert. $\mathbf{5 4}$ wird dann in einem zweiten Schritt mit zwei Äquivalenten n-Butyllithium wahrscheinlich über ein Vinyllithiumcarbenoid 55 unter Wanderung eines H-Atoms zum Alkin umgesetzt.

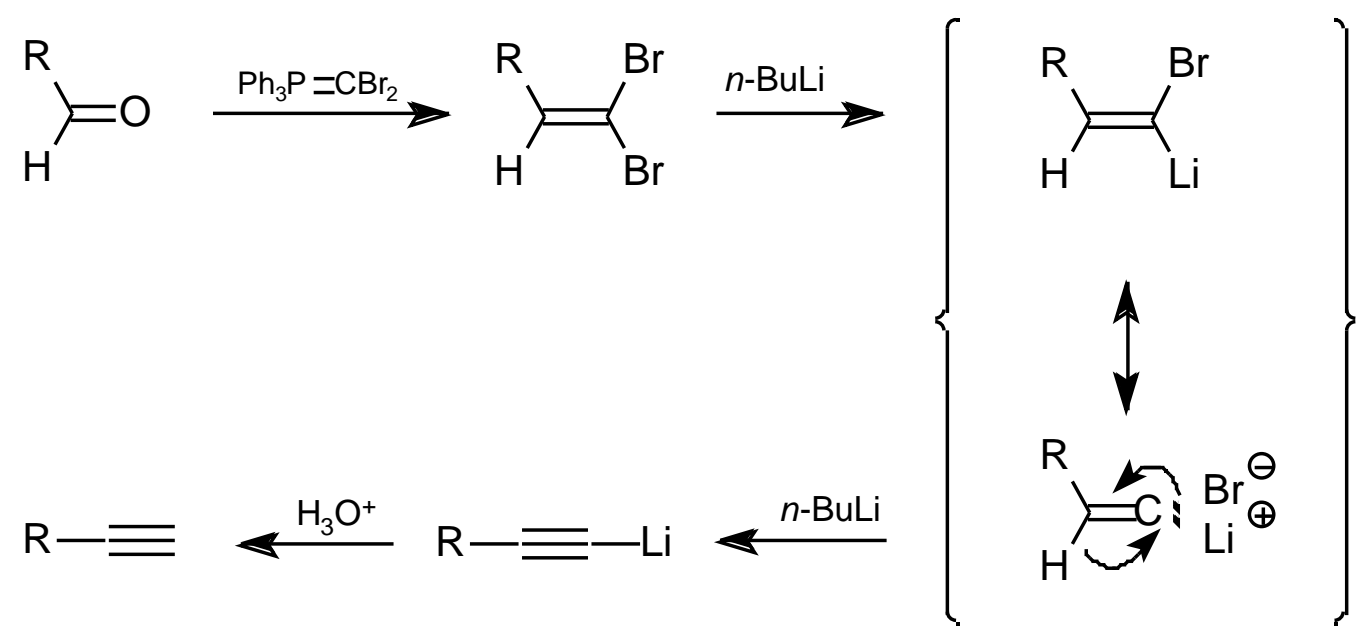

Schema 11. Mechanismus der Corey-Fuchs-Reaktion

Über die Corey-Fuchs-Reaktion gelang die Synthese von $\mathbf{5 3}$ in $71 \%$ Ausbeute aus dem Aldehyd 48.
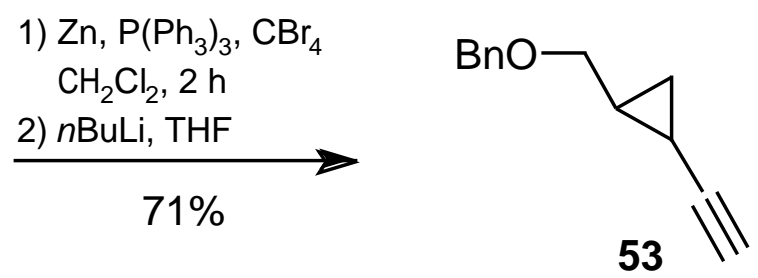

trans-1-Ethinyl-2-benzyloxymethylcyclopropan (53) ist somit in einer sechstufigen Synthese mit einer Gesamtausbeute von $31 \%$ erhältlich. 


\subsection{Versuch zur Darstellung von 1,2-Diethinylcyclopropan}

Setzt man beide Estergruppen in $\mathbf{2 2}$ gleichzeitig nach demselben Schema wie oben um, würde man in nur vier Stufen 1,2-Diethinylcyclopropan (56) erhalten. Im weiteren Verlauf der Synthese wäre eine Kupplung an beiden Ethinylgruppen möglich, wodurch man eine interessante Erweiterung für Folgereaktionen erhalten würde. Die Reduktion beider Estergruppen gelang zwar, aber aufgrund der hohen Polarität des entstehenden Diols 54 ließ sich das Produkt nur in 40\% Ausbeute isolieren. Die Swern-Oxidation führte in guten $86 \%$ Ausbeute zum bekannten Dialdehyd 55. Leider gelang es nicht, trans-1,2-Diethinylcyclopropan (56) über eine Corey-Fuchs-Reaktion zu synthetisieren. Nach der Eliminierung mit $n$-Butyllithium konnte kein Produkt isoliert werden.
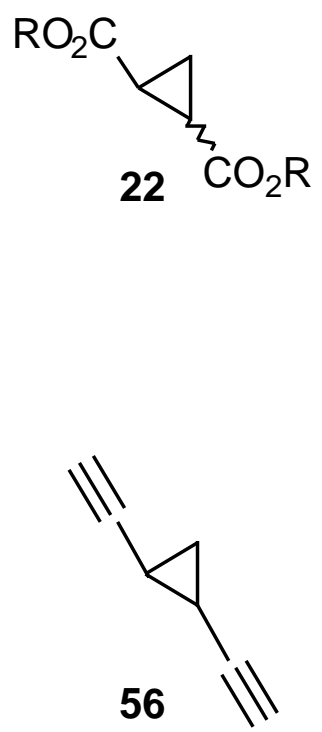

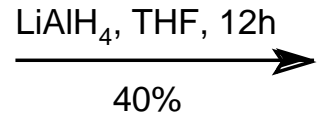

54

$86 \%\left\{\begin{array}{l}\text { 1) } \mathrm{DMSO},(\mathrm{COCl})_{2}, \mathrm{CH}_{2} \mathrm{Cl}_{2} \\ -70^{\circ} \mathrm{C}, 30 \mathrm{~min} \\ \text { 2) } \mathrm{NEt}_{3}\end{array}\right.$
1) $\mathrm{Zn}, \mathrm{PPh}_{3}, \mathrm{CBr}_{4}$

2) $n \mathrm{BuLi}$

$\mathrm{OHC}$

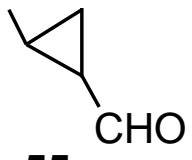

55

Schema 12. 


\subsection{Cyclopropanierungen an Pent-2-en-4-in-1-olen}

\subsubsection{Vorüberlegungen}

Die im Abschnitt 1.1 vorgestellte Synthese von geschütztem 1-Hydroxymethyl-2-ethinylcyclopropan (53) stellt einen guten Zugang zu diesen 1,2-substituierten Cyclopropanen dar. Es sollte nun versucht werden, eine kürzere Synthese zu finden und durch geeignete Methoden enantiomerenreines 24 zu erhalten, das als Kupplungspartner bei der Darstellung der 1,6-substituierten Endiine sehr attraktiv wäre.
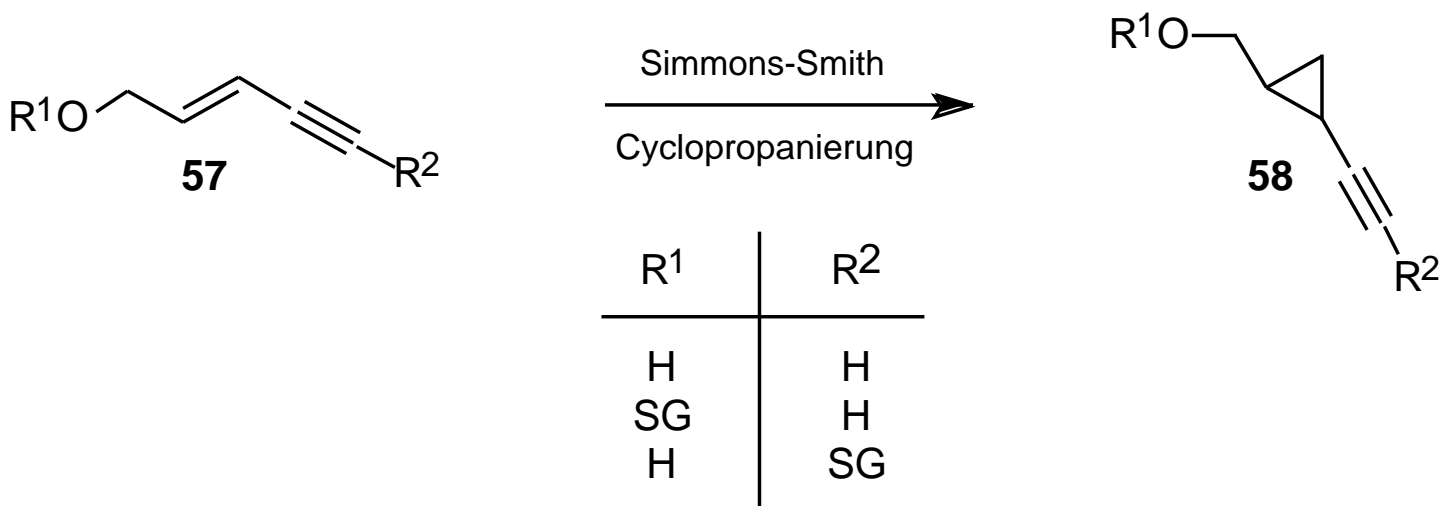

Schema 13. Simmons-Smith Cyclopropanierung an Eninen.

Der direkteste Vorläufer für 65 wäre Pent-2-en-4-in-1-ol (23) ${ }^{[23]}$, das in einer SimmonsSmith-Reaktion in nur einem Schritt zu 65 umgesetzt werden könnte ${ }^{[24]}$. Methoden zur asymmetrischen Cyclopropanierung werden zumeist an Allylakoholen mit einem enantiomerenreinen Auxiliar nach der Zink-Carbenoid-Methode durchgeführt ${ }^{[25]}$. Auch hierfür wäre 23 ein idealer Vorläufer. Erste Versuche zeigten, daß 23 sich nicht bei üblichen Reaktionsbedingungen cyclopropanieren ließ. Ein weiteres Problem stellt die gute Wasserlöslichkeit von 23 dar, die eine Aufarbeitung neben den vielen im Reaktiongemisch enthaltenen Metallsalzen erschwert. Weiterhin liegt die Polarität von Edukt und Produkt so nah beieinander, daß eine säulenchromatographische Reinigung nur schlecht möglich ist. Auch eine destillative Trennung von Edukt und Produkt sollte sich schwierig gestalten. Deshalb sollte 
die Darstellung so weit verbessert werden, daß man möglichst vollständigen Umsatz erhält. Die Reaktivitäts- und Trennprobleme sollten durch den Einsatz von geschützten Pent-2-en4-in-1-olen 57 verbessert werden.

\subsubsection{Darstellung und Schützung von Pent-2-en-4-in-1-ol}

Pent-2-en-4-in-1-ol (23) läßt sich gut und in großem Maßstab aus Epichlorhydrin (59) und Natriumacetylid (60) in flüssigem Ammoniak herstellen. 23 ist jedoch instabil und zersetzt sich langsam bei Raumtemperatur.

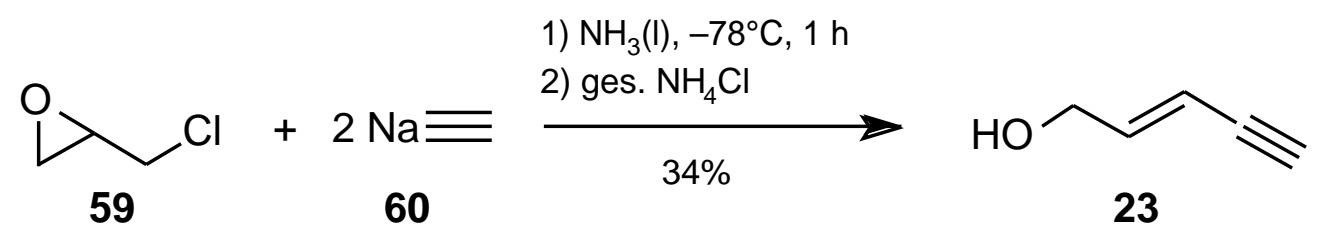

Um Cyclopropanierungsversuche nicht nur an freiem 23 durchführen zu können, wurden sowohl die Alkoholgruppe, als auch die endständige Acetylenfunktion geschützt. Die Acetylen-Funktion in 23 wurde mit der Trimethylsilylgruppe geschützt. Dazu muß man zunächst sowohl die Alkohol- als auch die Alkinylgruppe schützen. Bei der sauren Aufarbeitung entschützt man den Alkohol sofort wieder und erhält nur trimethylsilylgeschütztes 63. Es wurde versucht die Alkoholgruppe in $\mathbf{2 3}$ mit drei unterschiedlichen Gruppen zu schützen. Beim Versuch eine Acetatgruppe einzuführen, erhielt man bei den gewählten Bedingungen nur Polymer. Die tert-Butylgruppe wurde wegen der Instabilität von 23 nach einem milden Verfah- 
ren mit saurem Ionenaustauscher umgesetzt, reagierte jedoch nicht mit Isobuten. Erfolgreich und mit guten Ausbeuten verlief die Schützung mit tert-Butyldimethylsilylchlorid zu 64.

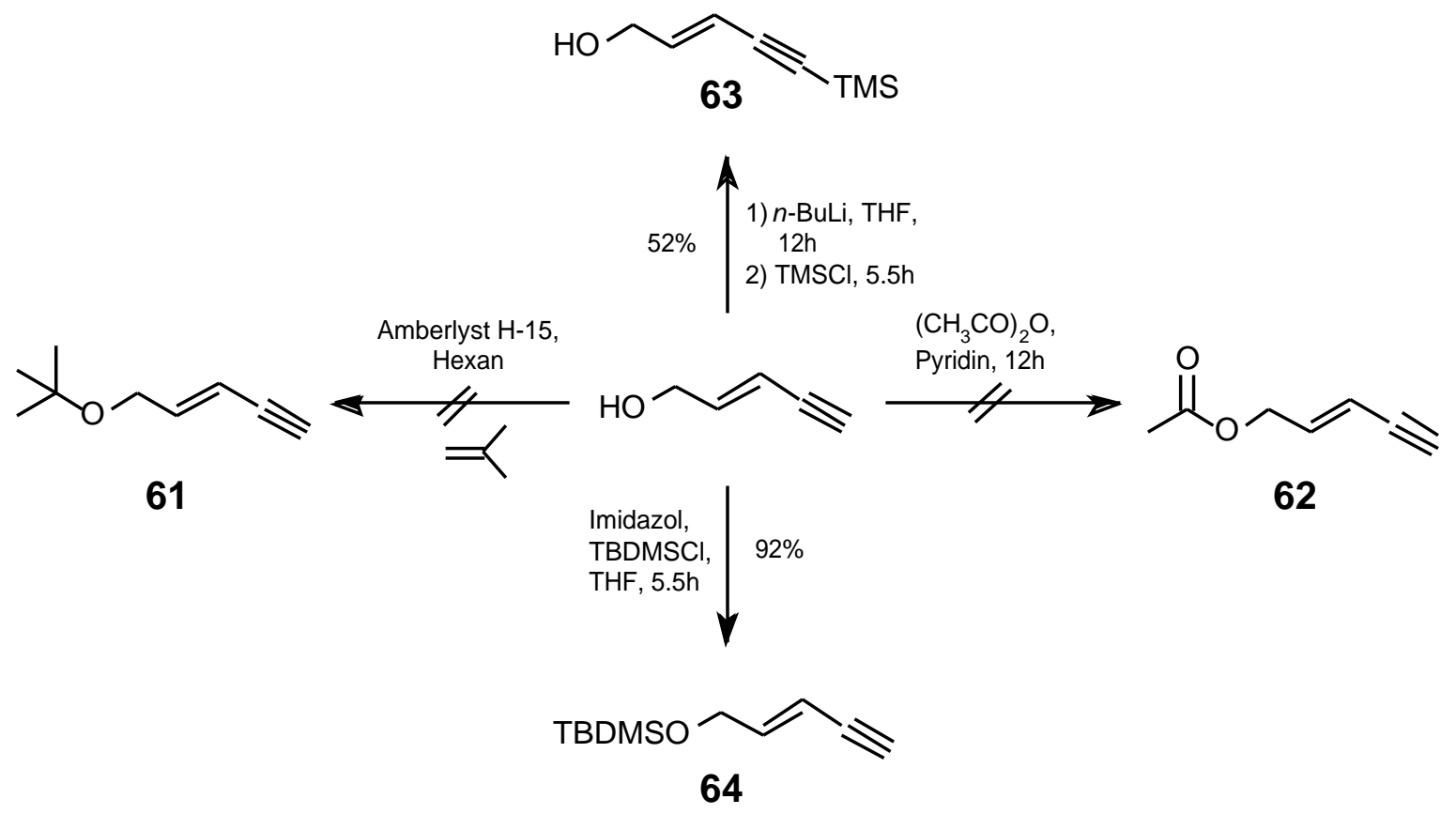

Schema 14. Schützungsversuche an Pent-2-en-4-in-1-ol

\subsubsection{Cyclopropanierungen}

Die Addition des Simmons-Smith Reagens an Alkene führt einstufig, stereoselektiv und stereospezifisch zu Cyclopropanen. Das Carbeniod wurde nach der Methode von Furukawa ${ }^{[24 c]}$ aus Diethylzink und Diiodmethan erzeugt. Die Zink-Alkyliodid-Spezies liegt in einem ähnlichen Gleichgewicht vor, wie man es von Grignardverbindungen als Schlenk-Gleichgewicht kennt. Es wurden nur Cyclopropanierungen mit Metallalkylen und Diiodmethan durchgeführt, weil sie, unterstützt durch chirale Auxiliare, am leichtesten diastereoselektiv an Allylalkohlen durchführbar sind. Die Reaktion wurde auf zwei unterschiedliche Arten durchgeführt. Bei Variante A setzte man zuerst den Allylalkohol mit Diethylzink in Dichlormethan um. Dabei addiert sich Diethylzink unter Freisetzung von Ethan an den Alkohol. Da- 
nach gab man Diiodmethan zu. Bei Variante B erzeugte man zuerst die aktive Zinkspezies aus Diethylzink und Diiodmethan in Dichlormethan und hielt sie durch Zugabe von THF in Lösung. Ohne THF bildet sich ein weißer Niederschlag und es konnte keine Umsetzung zum Cyclopropanierungsprodukt beobachtet werden.

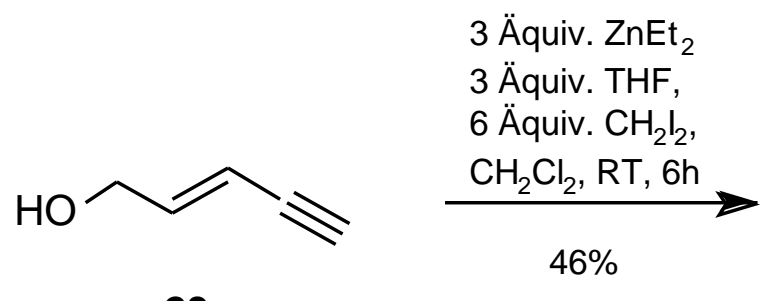

23

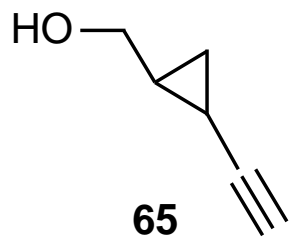

Zusammenfassend läßt sich sagen, daß nach Variante B 65 in 46\% Ausbeute isoliert werden konnte. Die Trennung von Edukt und Produkt gestaltete sich aufgrund des geringen Polaritätsunterschiedes schwierig. Eine destillative Trennung war nicht möglich.

Tab. 1: Cyclopropanierungen mit Diethylzink und Diiodmethan

\begin{tabular}{|c|c|c|c|c|c|c|c|}
\hline Variante & Edukt & $\begin{array}{c}\mathrm{ZnEt}_{2} \\
\text { (Äquiv.) }\end{array}$ & $\begin{array}{c}\mathrm{CH}_{2} \mathrm{I}_{2} \\
\text { (Äquiv.) }\end{array}$ & Temp. & Dauer & Edukt & $\begin{array}{l}\text { Produkt } \\
\text { (\% isoliert) }\end{array}$ \\
\hline A & 23 & 1.1 & 2.2 & $\mathrm{RT}$ & $18 \mathrm{~h}$ & & - \\
\hline A & 23 & 1.1 & 2.2 & reflux & $16 \mathrm{~h}$ & 3.5 & 1 \\
\hline A & 23 & 1.5 & 2.0 & reflux & $40 \mathrm{~h}$ & 5 & 1 \\
\hline A & 63 & 5.0 & 5.0 & RT & $16 \mathrm{~h}$ & 1 & $\begin{array}{c}4 \\
(23)\end{array}$ \\
\hline B & 23 & 1.5 & 2.0 & RT & $6 \mathrm{~h}$ & 1 & 1 \\
\hline B & 23 & 3.0 & 6.0 & RT & $6 \mathrm{~h}$ & 1 & $\begin{array}{c}5 \\
(46)\end{array}$ \\
\hline B & 64 & 2.0 & 4.0 & RT & $6 \mathrm{~h}$ & & - \\
\hline B & 64 & 3.0 & 6.0 & RT & $16 \mathrm{~h}$ & & - \\
\hline
\end{tabular}

Die beste Möglichkeit asymmetrische Cyclopropanierungen an Allylalkoholen durchzuführen, bietet heutzutage die Methode nach Charette[10]. Ein chiraler Boronsäureester, der sich leicht durch Kondensieren von $n$-Butylborsäureester und $(R, R)-(+)-N, N, N^{\prime}, N^{\prime}$-Tetramethyl- 
weinsäurediamid herstellen läßt, wird äquimolar zum Allylalkohol gegeben und mit Diethylzink und Diiodmethan umgesetzt. Das Schema 15 zeigt den Übergangszustand 66 der Cyclopropanierung nach Charette.

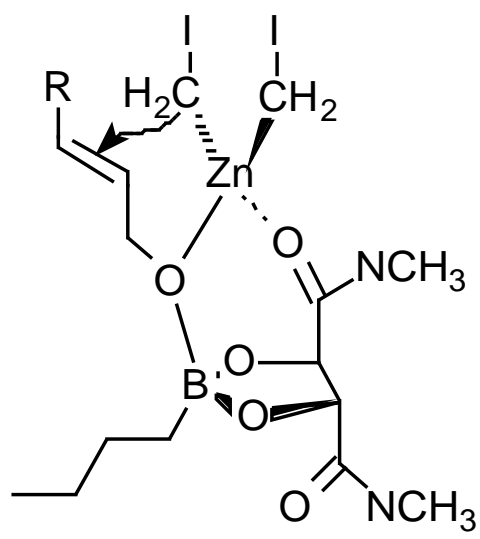

66

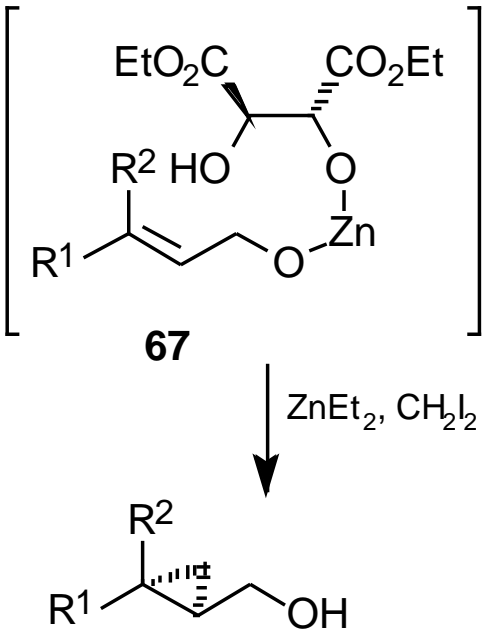

Schema 15. Übergangszustand der Cyclopropanierung nach Charette

Eine andere Variante wäre die Cyclopropanierung nach Fujisawa mit Weinsäurediethylester als chiralem Auxiliar. Dabei bildet sich intermediär die Struktur 67 aus. Zusätzlich wurde die Fujisawa-Variante[29] mit (R,R)-(+)-N,N,N',N'-Tetramethylweinsäurediamid durchgeführt, dabei wurde aber überhaupt kein Cyclopropanierungsprodukt gebildet. Alle Cyclopropanierungen wurden mit 23 durchgeführt, die Ergebnisse zeigt Tabelle 2. Eine weitere Methode zur asymmetrischen Cyclopropnierung wurde von Kobayashi[30] entwickelt. Sie verläuft mit katalytischen Mengen eines chiralen Disulfonamids. Da dabei im Prinzip unter ähnlichen Bedingungen wie bei der Cyclopropanierung nach Charette gearbeitet wird, wurde auf Cyclopropanierungen dieses Typs verzichtet, da dieses Verfahren vermutlich auch keine besseren Ergebnisse liefern würde. 
Tab. 2: Cyclopropanierungen mit chiralen Auxiliaren

\begin{tabular}{|c|c|c|c|c|c|}
\hline Auxiliar & $\begin{array}{c}\mathrm{ZnEt}_{2} \\
\text { (Äquiv.) }\end{array}$ & $\begin{array}{c}\mathrm{CH}_{2} \mathrm{I}_{2} \\
\text { (Äquiv.) }\end{array}$ & Dauer & Edukt & $\begin{array}{l}\text { Produkt } \\
\text { (\% isoliert) }\end{array}$ \\
\hline & 5.0 & 5.0 & $16 \mathrm{~h}$ & 3 & $\begin{array}{c}1 \\
(22)\end{array}$ \\
\hline & $2 \times 1.5$ & 2.0 & $4 \mathrm{~d}$ & 2 & $\begin{array}{c}1 \\
(23)\end{array}$ \\
\hline & $2 \times 1.5$ & 2.0 & $4 d$ & & - \\
\hline
\end{tabular}

Eine etwas ältere Methode zur asymmetrischen Cyclopropanierung verläuft über chirale Acetale. Ein ungesättigter Aldehyd wird mit einem Weinsäureester zum entsprechenden Acetal 69 umgesetzt. Die Cyclopropanierung erfolgt wieder über Diethylzink und Diiodmethan und liefert in guten Stereoselektivitäten Cyclopropane $\mathbf{7 0 .}$

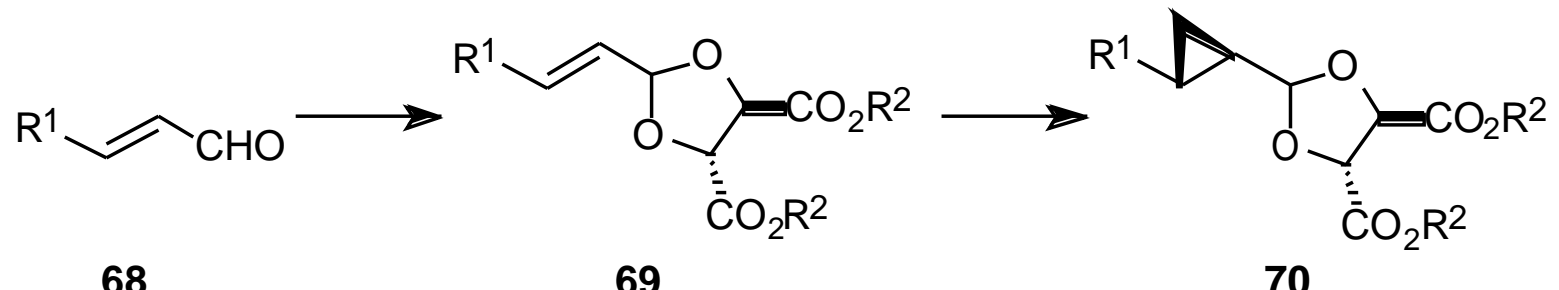

68

69

70

Schema 16. 
Diese Variante konnte leider nicht durchgeführt werden, da $\mathbf{2 3}$ sich weder mit dem DessMartin Reagens noch mit Mangandioxid zum Aldehyd oxidieren ließ.

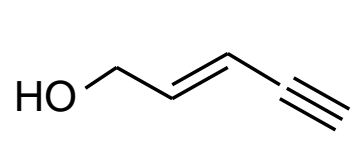

23
A) Dess-Martin

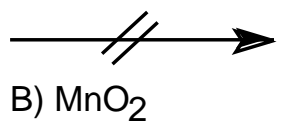

B) $\mathrm{MnO}_{2}$

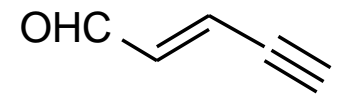

71

Schema 17. Oxidation nach Dess-Martin

Da die Cyclopropanierung mit dem Simmons-Smith Reagenz keine befriedigenden Ergebnisse lieferte, wurde eine alternative Methode mit Trimethylaluminium und Diiodmethan versucht ${ }^{[28]}$. Leider war hierbei überhaupt kein Umsatz zu beobachten.
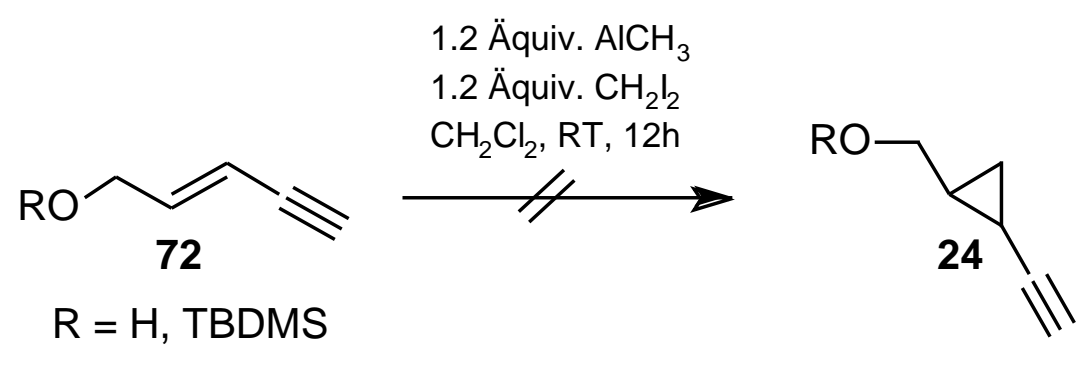

Schema 18. Cyclopropanierung mit Trimethylaluminium 


\section{Kupplungsreaktionen und Cyclopropanierungen an Kupplungsprodukten}

\subsection{Vorüberlegungen}

Die palladiumkatalysierte Kupplung von Alkinen 18 mit 1,2-Dihalogenalkenen 73 zu Endiinen kann entweder einstufig zu symmetrischen 1,6-substituierten Endiinen 75 oder zweistufig über Chlorenine 74 zu unsymmetrischen 1,6-substituierten Endiinen 20 führen $^{[33]}$. Es wurden beide Varianten erfolgreich durchgeführt und so Vorläufer für die weitere Synthese dargestellt.

$\mathrm{R}^{1}$

73

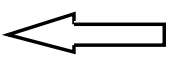

18

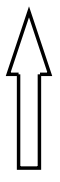

$R^{1}$

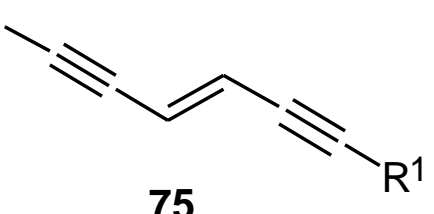

75

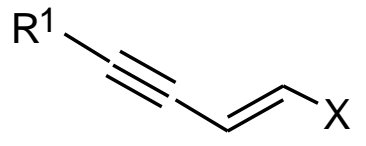

74
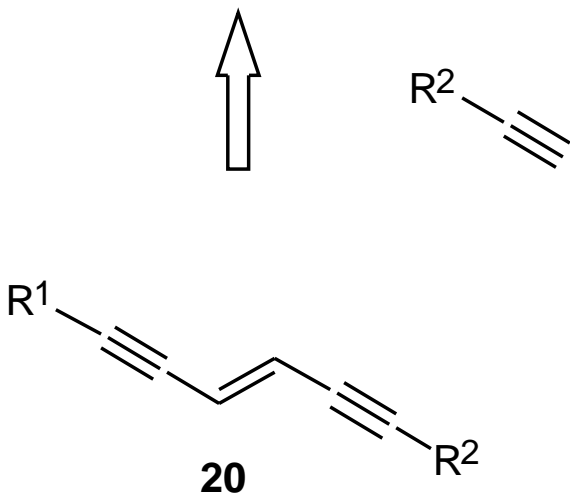

Schema 19. Darstellung von Endiinen

Kupplungen von terminalen Alkinen mit Alkenylbromiden oder -iodiden bezeichnet man als Stevens-Castro-Kupplung ${ }^{[34]}$. Man verwendet meistens mehrere Äquivalente eines primären, sekundären oder tertiären Amins und substöchiometrische Mengen Kupfer(I)-Iodid. Sie verlaufen palladium-katalysiert, wobei im ersten Schritt das Halogenalken 76 in einer oxidativen Addition an den Palladium(0)-Komplex 81 unter Ausbildung einer Palladium(II)Spezies 77 umgesetzt wird. Im zweiten Schritt findet eine Ummetallierung des Kupferace- 
tylids 78 statt, das sich in einem vorgelagerten Schritt aus dem Alkin, dem Amin und Kupfer(I)-iodid bildet. Durch einen Ligandenaustausch an $\mathbf{7 7}$ wird aus der Alkinylkupfer- eine Alkinylpalladium-Verbindung 79. Durch eine reduktive Eliminierung wird das Kupplungsprodukt $\mathbf{8 0}$ freigesetzt und der nullwertige Palladium-Komplex $\mathbf{8 1}$ kann wieder mit einem 76 reagieren.

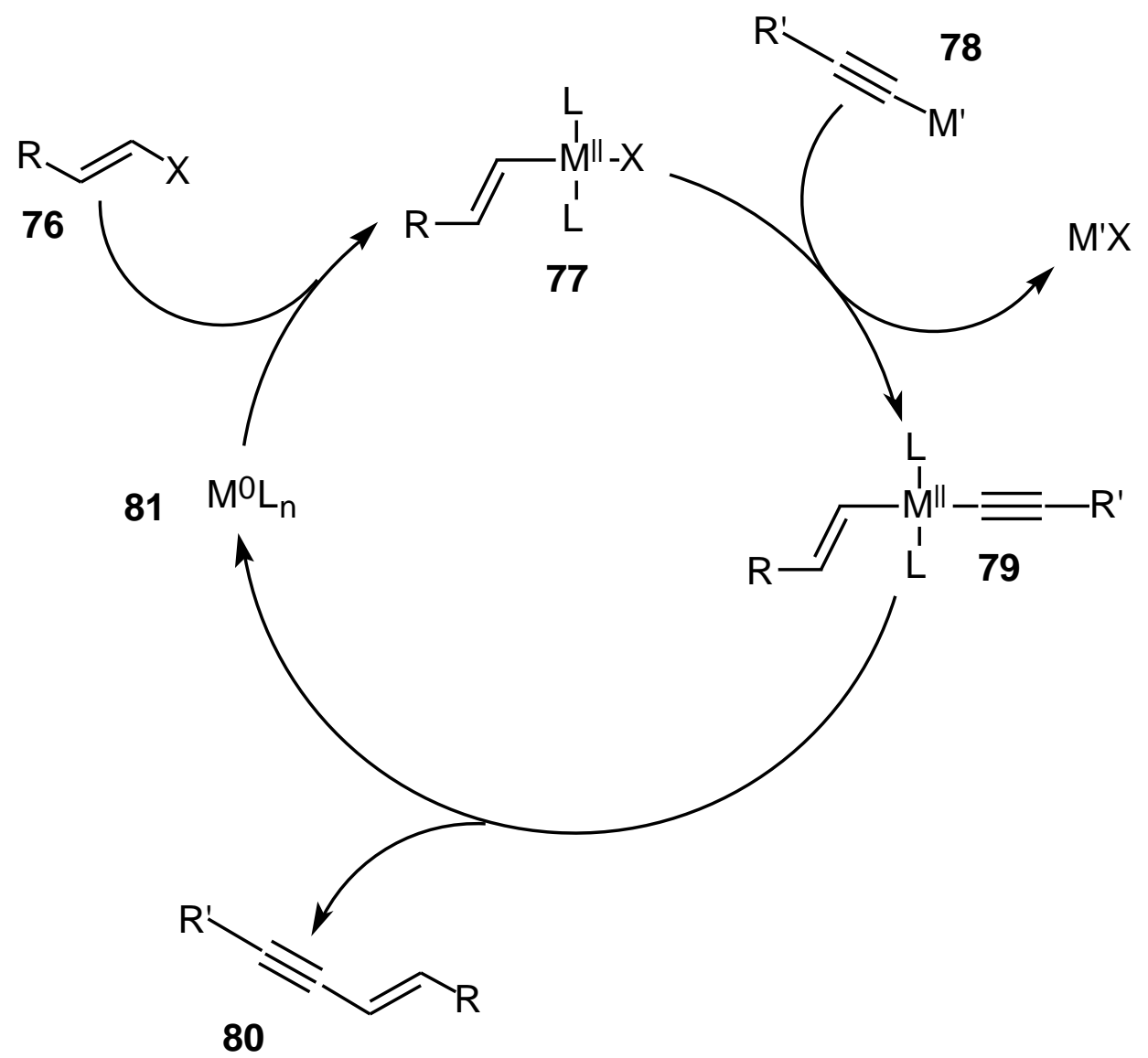

Schema 20. Reaktionsmechanismus der Stevens-Castro-Kupplung 
Bei Endiinen mit endständigen Allylalkoholfunktionen 82 wurde die Cyclopropylgruppe direkt nach der Kupplung zum Endiin eingeführt, da die anschließende Dihydroxylierung nicht selektiv an der zentralen Doppelbindung durchgeführt werden kann.

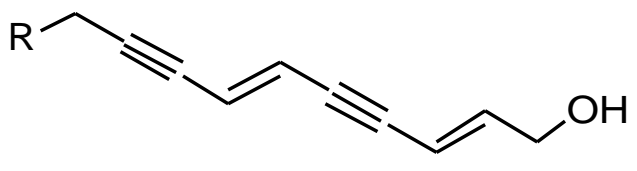

82

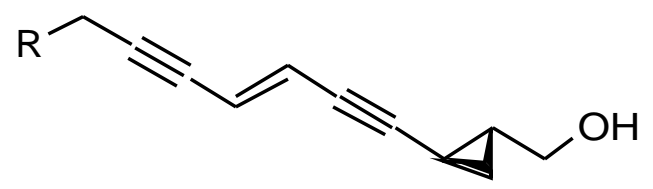

83

Schema 21. Cyclopropanierung endständiger Allylalkohole 


\subsection{Palladium-katalysierte Kupplung von Acetylenen zu Endiinen}

Es gibt viele Methoden $s p^{2}$ - und $s p$-hybridisierte Kohlenstoffzentren palladium-katalysiert zu Eninen zu kuppeln, aber nur wenige Möglichkeiten aus 1,2-Diaholgenalkenen und Alkinen in einer einstufigen Synthese symmetrische Endiine darzustellen ${ }^{[35]}$. Ein Weg ist die Umsetzung von Alkinylzinkchloriden mit Alkinen und 1,2-Dibromethen unter Palladium(0)-Katalyse. Das Alkinylzinkchlorid 84 wird durch Lithiierung terminaler Alkine 18 mit n-Butyllithium und anschließendem Metall-Metall-Austausch mit Zink(II)-chlorid in situ erzeugt und mit 1,2-Dibromethen und Tetrakis-(triphenylphosphin)-palladium(0) zu den trans-Endiinen 75 umgesetzt. Die Synthese zweier Endiine gelang in zufriedenstellenden Ausbeuten (Schema 22). Mit einem zweiten aciden Proton in der Alkin-Komponente, konnte kein Endiin isoliert werden.

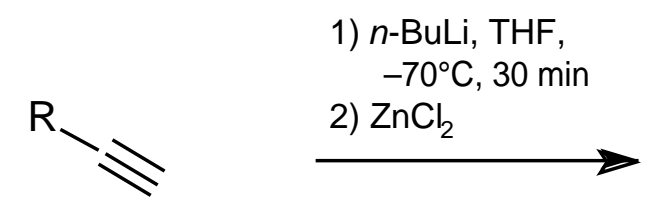

18
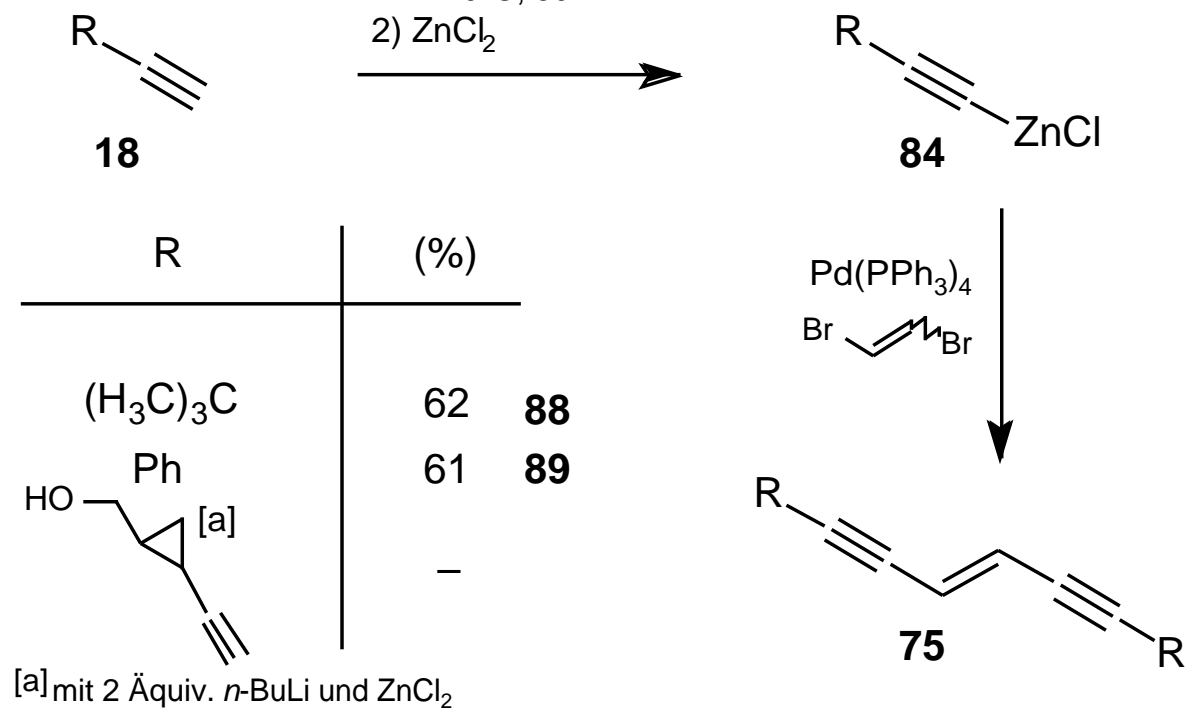

Schema 22. Kupplungen zu symmetrischen Endiinen

In der Literatur sind Kupplungen von verschiedenartig substituierten terminalen Alkinen mit trans-1,2-Dichlorethen unter Tetrakis-(triphenylphosphin)-palladium(0)-Katalyse und Zusatz von Kupfer(I)-Iodid und $n$-Butylamin mit sehr guten Ausbeuten (85-98\%) beschrieben ${ }^{[36]}$. Es wurde versucht, unter diesen oder ähnlichen Bedingungen verschiedene Alkine 
umzusetzen. Dabei wurden jedoch entweder gar keine Kupplungsprodukte oder nur Gemische aus Halogeneninen, Diinen und Endiinen in schlechten Ausbeuten isoliert.

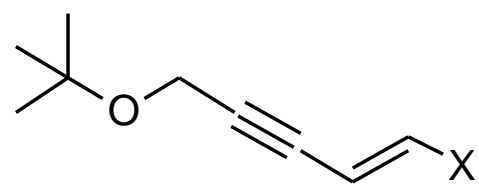

85

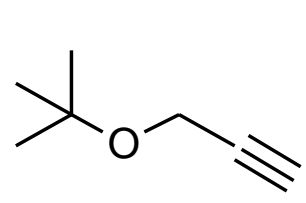

84
Pd-Kat., ${ }^{x} \boldsymbol{D}^{x}$ $n \mathrm{BuNH}_{2}$, Cul
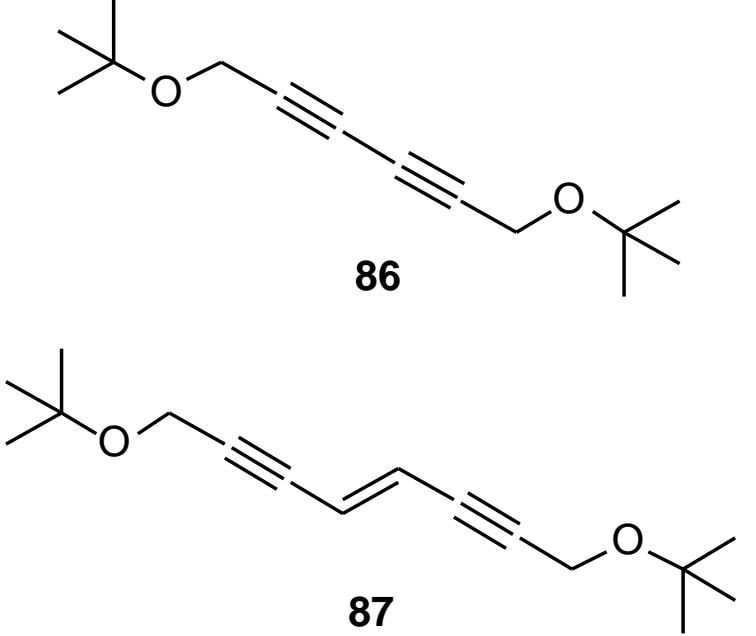

Auch der Einsatz des reaktiveren trans-1,2-Dibromethens und der anderen Basen wie Diethyloder Triethylamin gab in keinem Fall die gewünschten symmetrischen Endiine. Tabelle 3 zeigt die Ergebnisse von Kupplungen mit $\mathbf{8 4}$ und die Produktverhältnisse.

Tab. 3: Kupplung von 84.

\begin{tabular}{ccccccc}
\hline Alken & Katalysator & $\begin{array}{c}\text { Temp. } \\
{\left[{ }^{\circ} \mathrm{C}\right]}\end{array}$ & $\begin{array}{c}\text { Dauer } \\
{[\mathrm{h}]}\end{array}$ & $\begin{array}{c}\text { Enin } \\
(\%)\end{array}$ & $\begin{array}{c}\text { Diin } \\
(\%)\end{array}$ & $\begin{array}{c}\text { Endiin } \\
(\%)\end{array}$ \\
\hline $\mathrm{Cl}$ & $\mathrm{Pd}\left(\mathrm{PH}_{3}\right)_{2} \mathrm{Cl}_{2}$ & $\mathrm{RT}$ & 22 & 30 & 7 & 7 \\
$\mathrm{Br}$ & $\mathrm{Pd}\left(\mathrm{PH}_{3}\right)_{2} \mathrm{Cl}_{2}$ & $\mathrm{RT}$ & 22 & 8 & 15 & - \\
$\mathrm{Br}$ & $\mathrm{Pd}(\mathrm{PPh})_{4}$ & $\mathrm{RT}$ & 22 & 37 & 4 & 1.4 \\
\hline
\end{tabular}


2.3. Darstellung von Chloreninen aus Acetylenen und trans-1,2-Dichlorethen

Um unsymmetrische Endiine zu erhalten, kann man zunächst 1,2-Dichlorethen mit Alkinen zu Chloreninen kuppeln. In der Literatur findet man eine Vielzahl an Beispielen dieses Kupplungstyps ${ }^{[37]}$. Der beste Katalysator für diese Reaktion ist Tetrakis-(triphenylphosphin)-palladium(0). Verwendet man Piperidin als Base, erreicht man mit trans-1,2-Dichlorethen am schnellsten vollständigen Umsatz zum Produkt. Das Dichlorethen setzt man im Überschuß (5 Äquiv.) ein, um die Bildung von Endiinen zu unterbinden. Optimale Ausbeuten erreicht man mit Benzol als Lösungsmittel, in THF sind die Ausbeuten deutlich niedriger.

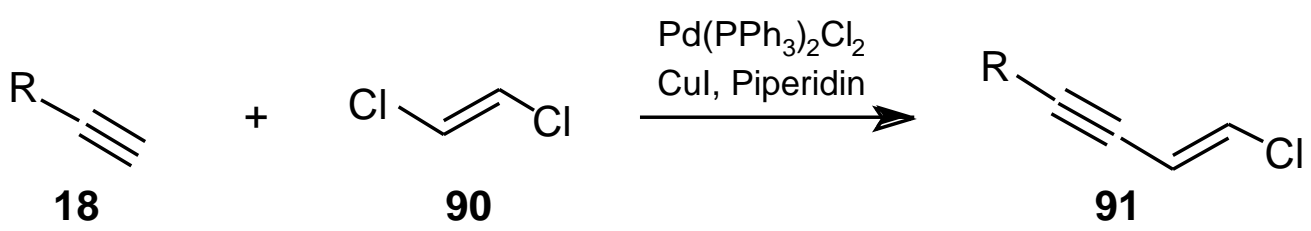

Die Kupplungen zu Chloreninen konnten mit einer Reihe von Alkinen in guten bis sehr guten Ausbeuten durchgeführt werden. Die Reaktionen wurden nicht wie in der Literatur beschrieben bei $20{ }^{\circ} \mathrm{C}$ durchgeführt, sondern bei $0{ }^{\circ} \mathrm{C}$ durch Zugabe des Kupfer(I)-iodids gestartet und dann langsam auf Raumtemperatur erwärmt, da sie sehr exotherm verliefen und $\mathbf{9 0} \mathrm{zu}$ sieden begann. Die Reaktionen waren nach 1.5-3 h beendet. Die Ergebnisse der Umsetzungen mit unterschiedlichen Alkinen zeigt Tabelle 4.

Kupplungen mit tert-Butoxy-2-propin (84) zum Chlorenin 92 wurden sowohl mit $n$-Butylamin in 56\%, als auch mit Piperidin als Base in $82 \%$ Ausbeute durchgeführt.

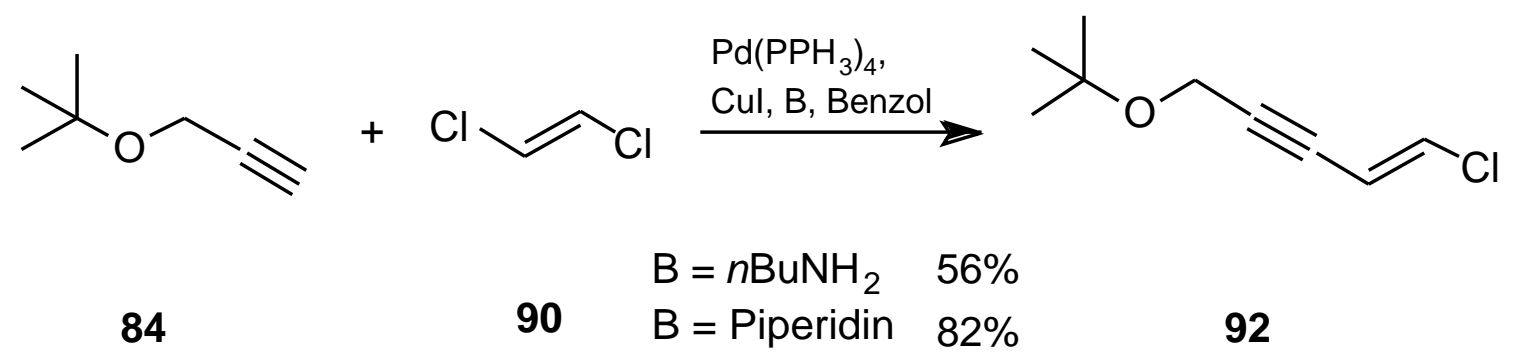

Schema 23. 
Tab. 4: Darstellung von Chloreninen

\begin{tabular}{cccccc}
\hline & $\begin{array}{c}\text { Dauer } \\
{[\mathrm{h}]}\end{array}$ & $\begin{array}{c}\mathrm{Pd}(\mathrm{PPh})_{4} \\
(\mathrm{~mol} \%)\end{array}$ & $\begin{array}{c}\text { CuI } \\
(\mathrm{mol} \%)\end{array}$ & Produkt & $\begin{array}{c}\text { Ausbeute } \\
(\%)\end{array}$ \\
\hline 2.5 & 2.8 & 5.6 & 93 & 82 \\
\hline
\end{tabular}


2.4. Palladium-katalysierte Kupplung von Chloreninen mit Acetylenen

Die zweite Kupplung zu Endiinen verläuft in sehr guten Ausbeuten mit einer Reihe von Chloreninen. Dabei verlief die Reaktion besser mit Bis(triphenylphosphin)palladium(II)chlorid als mit Tetrakis(triphenylphosphin)palladium(0). Die Base Piperidin wurde als Lösungsmittel verwendet ${ }^{[38]}$.

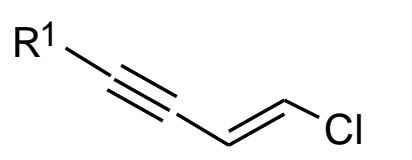

91

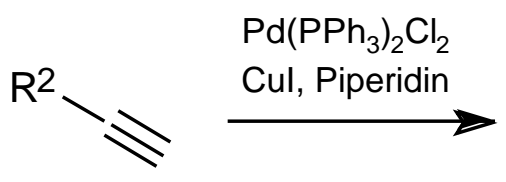

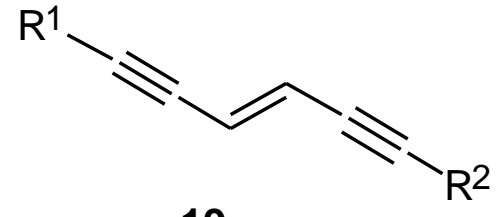

19

Schema 24. Darstellung unsymmetrischer Endiinen aus Chloreninen

Die Kupplung verschiedener Chlorenine 91 mit trans-1,2-Dichlorethen führte in guten Ausbeuten zu 1,6-substituierten Endiinen 19. Die Ergebnisse der Umsetzungen zeigt Tabelle 5. 
Tab. 5: Kupplung von Chloreninen zu Endiinen

\begin{tabular}{|c|c|c|c|c|c|c|}
\hline $\mathrm{R}^{1}$ & $\mathrm{R}^{2}$ & $\begin{array}{c}\text { Dauer } \\
{[\mathrm{h}]}\end{array}$ & $\begin{array}{c}\mathrm{Pd}(\mathrm{PPh})_{2} \mathrm{Cl}_{2} \\
\quad(\mathrm{~mol} \%)\end{array}$ & $\begin{array}{c}\mathrm{CuI} \\
(\mathrm{mol} \%)\end{array}$ & $\begin{array}{l}\text { Pro- } \\
\text { dukt }\end{array}$ & $\begin{array}{l}\text { Aus- } \\
\text { beute } \\
(\%)\end{array}$ \\
\hline 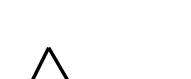 & & 2.0 & 4.0 & 9.3 & 98 & 95 \\
\hline & & 2.5 & 4.0 & 9.3 & 99 & 55 \\
\hline & & 1.75 & 5.7 & 8.1 & 100 & 82 \\
\hline & & 1.5 & 4.4 & 10 & 101 & 95 \\
\hline & & 1.5 & 5.8 & 12 & 102 & 68 \\
\hline
\end{tabular}

In der Literatur ist auch eine Eintopfvariante zur Darstellung der unsymmetrischen Endiine zu finden ${ }^{[39]}$. Hierbei wird zuerst das Chlorenin dargestellt, alle flüchtigen Komponenten im Vakuum entfernt und der Rückstand in Piperidin aufgenommen und mit neuem Katalysator und dem zweiten Alkin zur Reaktion gebracht. Mit den Alkinen $\mathbf{8 4}$ und 23 konnte auf diesem Wege das entsprechende Endiin nur in Spuren hergestellt werden.

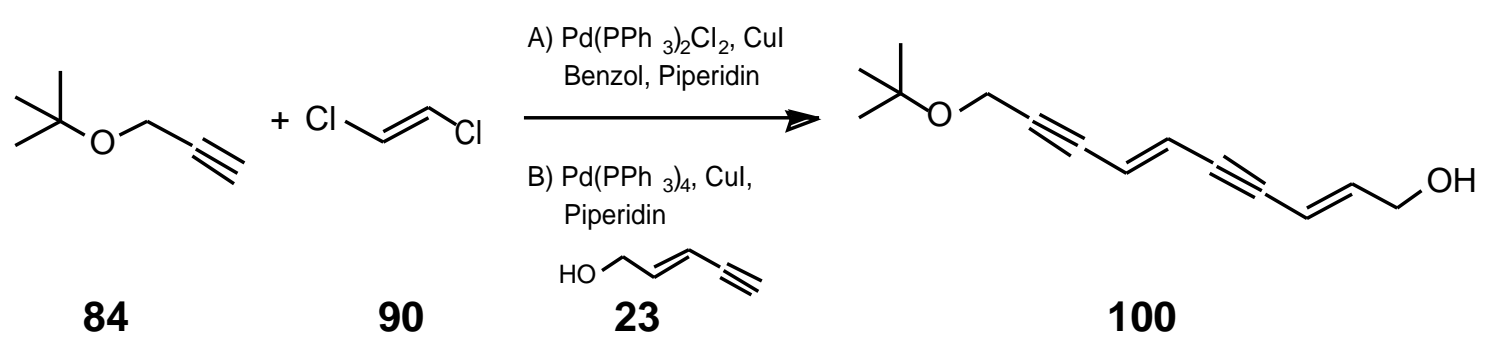


2.5. Cyclopropanierungen an endständigen Allylalkohol-Funktionen ungesättigter Vorläufer

Bei Kupplungen mit Pent-2-en-4-in-1-ol (23) entstehen ungesättigte Ketten mit endständigen Allylalkohol-Funktionen (82). Bevor weitere Transformationen an diesen Endiinen vorgenommen werden können, muß man sie nach einer in Abschnitt 1.3 vorgestellten Methode cyclopropanieren. Die Reaktionen sollten auch selektiv an der Allylalkohol-Doppelbindung

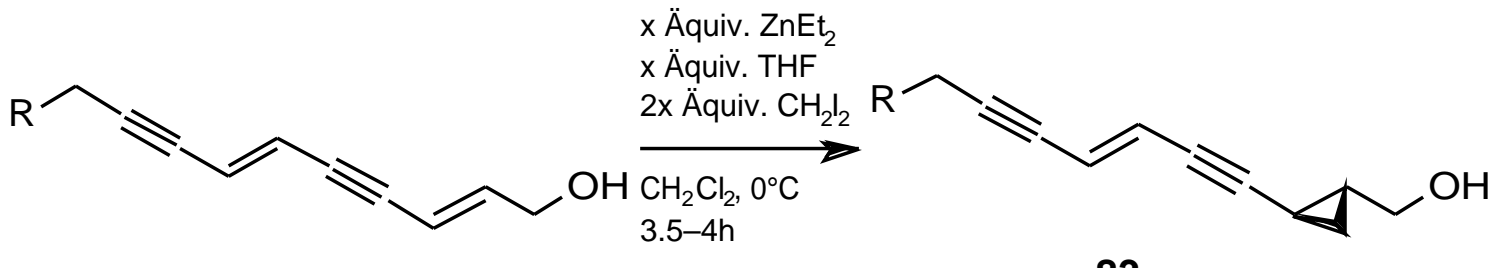

82

$30-54 \%$

83

Schema 25. Cyclopropanierung der endständigen Allylalkoholfunktion

stattfinden, da durch Koordination des Zinkcarbenoids am Sauerstoff bevorzugt diese Doppelbindung angegriffen wird.

Verschiedene mehrfachungesättigte Allylalkohole $\mathbf{8 3}$ konnten auf diese Weise, allerdings nur in mäßigen Ausbeuten, cyclopropaniert werden. Es war zu beobachten, daß die Ausbeuten bei Umsetzungen bei Raumtemperatur deutlich zurückgehen.

Tab. 6: Cyclopropanierungen endständiger Allylalkohol-Funktionen

\begin{tabular}{|c|c|c|c|c|c|c|}
\hline $\mathrm{R}$ & $\begin{array}{c}\text { Dauer } \\
{[\mathrm{h}]}\end{array}$ & $\begin{array}{c}\mathrm{ZnEt}_{2} \\
\text { (Äquiv.) }\end{array}$ & $\begin{array}{c}\mathrm{CH}_{2} \mathrm{I}_{2} \\
\text { (Äquiv.) }\end{array}$ & Produkt & $\begin{array}{l}\text { Edukt } \\
(\%)\end{array}$ & $\begin{array}{c}\text { Produkt } \\
(\%)\end{array}$ \\
\hline & 3.75 & 3 & 6 & 103 & 13 & 50 \\
\hline & 3.5 & 3 & 6 & 104 & 2.7 & 54 \\
\hline & 4.0 & 4 & 4 & 105 & - & 30 \\
\hline
\end{tabular}




\section{1,2-Dihydroxylierungen an Endiinen}

\subsection{Vorüberlegungen}

Der erste Schritt zur Transformation der 1,6-substituierten Endiine 19 ist eine 1,2-Dihydroxylierung. Diese sollte nach Sharpless zu enantiomerenreinen 1,2-Diolen oder anderen geeigneten Methoden cis-selektiv durchgeführt werden.

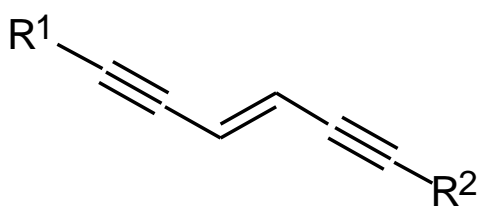

19

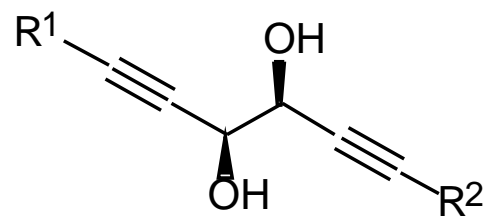

20
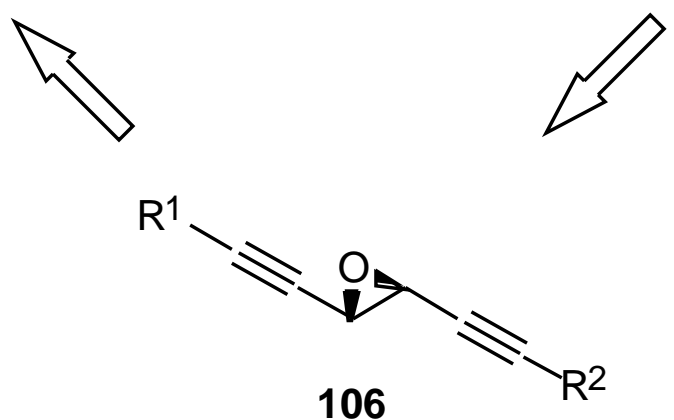

Schema 26.

Über die Alkoholgruppen an $\mathbf{2 0}$ sollten die weiteren Reaktionsschritte gesteuert und selektiv Folgeprodukte entstehen.

Schon in der Diplomarbeit zeigte sich, daß Endiine sich nicht unter normalen Sharpless-Bedingungen mit dem Standard-AD-mix umsetzen. Die Dihydroxylierung sollte nun optimiert werden oder eine andere Methode zur cis-Hydroxylierung Anwendung finden. 


\subsection{Dihydroxylierungen nach Sharpless}

Die Sharpless-Dihydroxylierung ${ }^{[40]}$ ist ein einfach durchzuführendes Standardverfahren, um Alkene in enantiomerenreine Diole zu überführen. Katalytische Mengen Osmium, ein chiraler Ligand und ein Reoxidationssystem aus Kaliumhexacyanoferrat und Kaliumcarbonat bilden das Katalysatorsystem und sind als AD-mix vorgefertigt zu kaufen. Die Reaktion läuft in einem Zweiphasensystem aus Wasser und tert-Butanol ab. In der wässrigen Phase wird das Osmium(VI) 107 von einem basischen Kaliumcarbonat/Eisenhexacyanoferrat-System wieder zum Osmium(VIII) 108 reoxidiert. In der organischen Phase findet die Dihydroxylierung statt. Dabei bildet sich aus Osmiumtetroxid 109 dem Alken 110 und dem chiralen

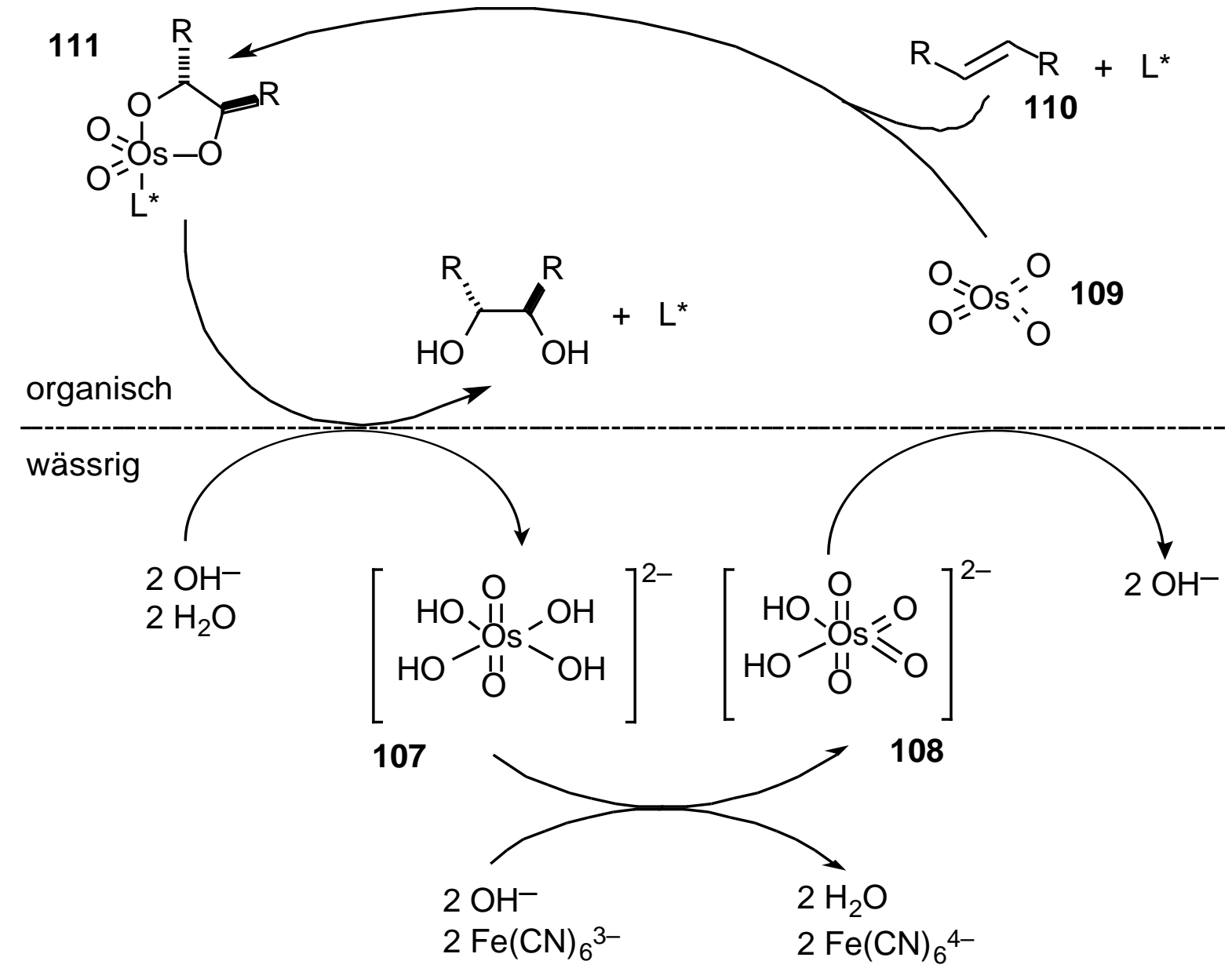

Schema 27. Reoxidationssystem der Sharpless-Dihydroxylierung 
Liganden ein Osmium(VI)-Glykolat 111. Es ist noch nicht endgültig geklärt, ob sich 111 in einer [2 + 2]-Cycloaddition oder in einer 1,3-Dipolaren Cycloaddition bildet. Die Hydrolyse von 111 wird durch den Zusatz von Methansäuresulfonamid beschleunigt, deshalb wird bei Alkenen, die mehr als einfach substituiert sind Methansäuresulfonamid zugesetzt.

Es wurden im Laufe der Zeit mehrere Liganden entwickelt. Der am häufigsten verwendete ist (DHQD) ${ }_{2}$ PHAL 113, er wird auch zur Umsetzung von Endiinen favorisiert. Der Ligand

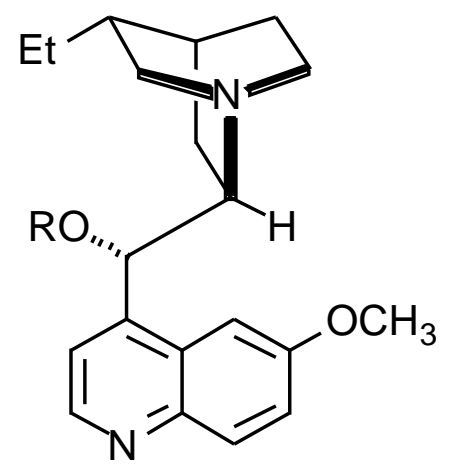

Dihydrochinidin $(\mathrm{R}=\mathrm{H})$ : Alk*

112

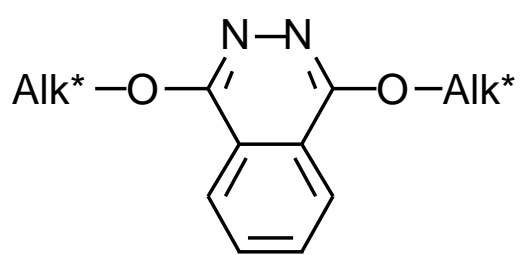

Phthalazine (PHAL) Liganden

113

bildet mit dem Osmiumtetroxid eine enzymartige Bindungstasche aus, in die das Alken nur in einer bevorzugten Ausrichtung hineinpaßt.

Der Vorteil der Dihydroxylierung ist die hohe Variationsbreite des eingesetzten Alkens. Allgemein sind die Ausbeuten niedriger, je elektronenärmer die Doppelbindung ist. Man kann einfach- bis vierfach-substituierte ${ }^{[41]}$ Alkene einsetzen. Reaktionen mit Eninen sind bekannt und führen je nach Substituenten zu vernünftigen Ausbeuten ${ }^{[42]}$.

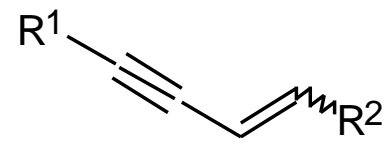

114

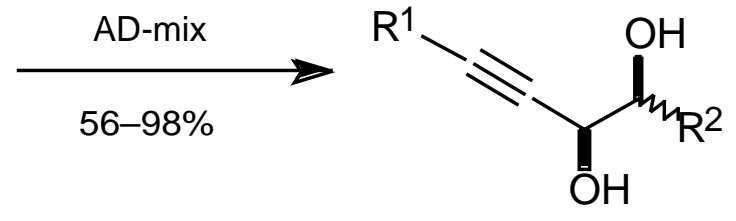

115

Schema 28. Dihydroxylierung von Eninen 
Schlecht reagierende Alkene kann man auch mit 1 mol\% Osmium und 2 mol\% Ligand 24 h lang bei Raumtemperatur umsetzen. Endiine lassen sich nur schlecht umzusetzen und reagieren nur mit größeren Mengen Osmium und Ligand.

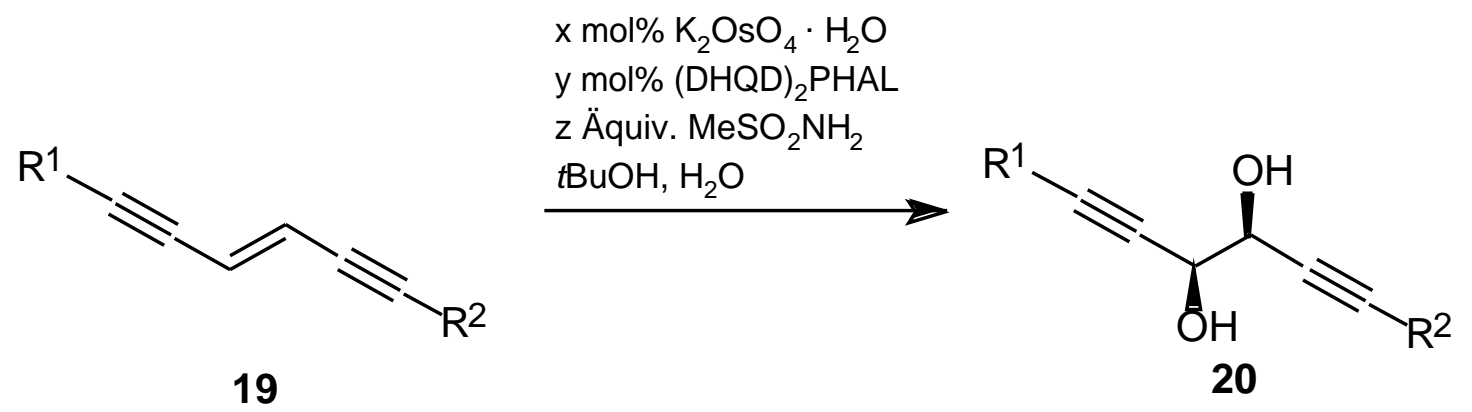

Schema 29. Reaktionsmischung für Dihydroxylierungen

Da Endiine 19 sich mit dem normalen AD-mix in nur 5\% Ausbeute zu den Diolen 20 umsetzen, wurden Optimierungsversuche mit speziell zusammengesetzten Katalysatormischungen durch geführt. Die Ergebnisse dieser Versuche zeigt die Tabelle 7. Die Ausbeuten lagen im allgemeinen um die 20\%, nur bei Einsatz von mehreren Molprozent Kaliumosmat und Ligand konnten 49\% Ausbeute erreicht werden.

Tab. 7: Dihydroxylierungen an Endiinen

\begin{tabular}{ccccccccc}
\hline $\begin{array}{c}\text { En- } \\
\text { diin }\end{array}$ & $\begin{array}{c}\mathrm{Os} \\
(\mathrm{mol} \%)\end{array}$ & $\begin{array}{c}\text { Ligand } \\
(\mathrm{mol} \%)\end{array}$ & $\begin{array}{c}\mathrm{MeSO}_{2} \mathrm{NH}_{2} \\
(\ddot{\mathrm{Aq}} \text { quiv. })\end{array}$ & Temp. & $\begin{array}{c}\text { Dauer } \\
{[\mathrm{h}]}\end{array}$ & $\begin{array}{c}\text { Edukt } \\
(\%)\end{array}$ & $\begin{array}{c}\text { Pro- } \\
\text { dukt } \\
(\%)\end{array}$ & $\begin{array}{c}\text { Pro- } \\
\text { dukt }\end{array}$ \\
\hline $\mathbf{8 8}$ & 0.2 & 1.0 & 1 & $0-\mathrm{RT}$ & 16 & 64 & 5 & $\mathbf{1 1 6}$ \\
$\mathbf{8 8}$ & 0.2 & 1.0 & 3 & $\mathrm{RT}$ & 3 & 60 & 5 & $\mathbf{1 1 6}$ \\
$\mathbf{8 8}$ & 5 & 10 & 5 & $\mathrm{RT}$ & 24 & 42 & 49 & $\mathbf{1 1 6}$ \\
$\mathbf{1 0 1}$ & 1 & 2 & 5 & $0-\mathrm{RT}$ & 24 & 55 & - & $\mathbf{1 1 7}$ \\
$\mathbf{1 0 0}$ & 0.2 & 1 & 2 & 55 & 24 & 30 & - & $\mathbf{1 1 8}$ \\
$\mathbf{9 8}$ & 1 & 2 & 2 & $\mathrm{RT}$ & 24 & 53 & 18 & $\mathbf{1 1 9}$ \\
$\mathbf{9 8}$ & 1 & 2 & 4 & RT & 24 & 51 & 27 & $\mathbf{1 1 9}$ \\
$\mathbf{9 8}$ & 1 & 2 & 6 & RT & 24 & 44 & 21 & $\mathbf{1 1 9}$ \\
$\mathbf{9 8}$ & 2 & 2 & 4 & RT & 24 & 35 & 28 & $\mathbf{1 1 9}$ \\
\hline
\end{tabular}


Tab. 7: Dihydroxylierungen an Endiinen

\begin{tabular}{ccccccccc}
\hline $\begin{array}{c}\text { En- } \\
\text { diin }\end{array}$ & $\begin{array}{c}\text { Os } \\
(\mathrm{mol} \%)\end{array}$ & $\begin{array}{c}\text { Ligand } \\
(\mathrm{mol} \%)\end{array}$ & $\begin{array}{c}\mathrm{MeSO}_{2} \mathrm{NH}_{2} \\
(\text { Äquiv. })\end{array}$ & Temp. & $\begin{array}{c}\text { Dauer } \\
{[\mathrm{h}]}\end{array}$ & $\begin{array}{c}\text { Edukt } \\
(\%)\end{array}$ & $\begin{array}{c}\text { Pro- } \\
\text { dukt } \\
(\%)\end{array}$ & $\begin{array}{c}\text { Pro- } \\
\text { dukt }\end{array}$ \\
\hline $\mathbf{9 8}$ & 1 & 3 & 4 & $\mathrm{RT}$ & 24 & 43 & 33 & $\mathbf{1 1 9}$ \\
98 & 2 & 3 & 4 & RT & 24 & 62 & 22 & $\mathbf{1 1 9}$ \\
98 & 1 & 2 & 3 & RT & 24 & 50 & 26 & $\mathbf{1 1 9}$ \\
99 & 1 & 2 & 4 & RT & 24 & 59 & 18 & $\mathbf{1 2 0}$ \\
\hline
\end{tabular}




\subsection{Dihydroxylierungen mit Rutheniumtetroxid}

Mit Rutheniumtetroxid lassen sich unterschiedliche Substrate auf vielfältige Weise oxidieren. So kann man beispielsweise Benzylether 121 zu Benzylestern 122[43], endständige Alkene 123 zu um einen Kohlenstoff verkürzte Carbonsäuren $124^{\text {[44] }}$ und Isobutyl-Einheiten $125 \mathrm{zu}$ Hydroxyketonen 126 ${ }^{[45]}$ oxidieren.<smiles>CCCCCCOCc1ccccc1</smiles>

121<smiles>C=CCCCCCCCCC</smiles>

123<smiles>CC(=O)OCC=C(C)C</smiles>

125
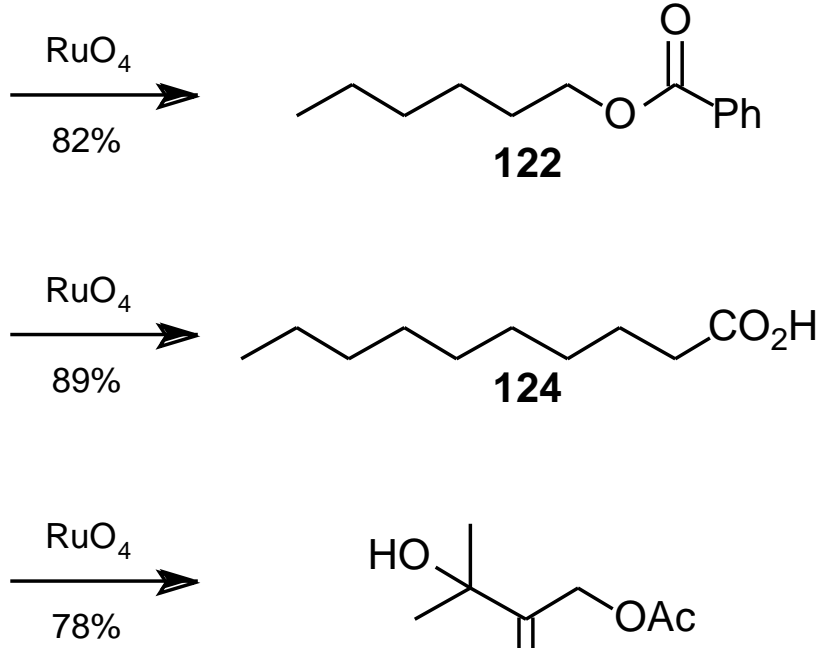<smiles>CCOCC(=O)C(C)(C)O</smiles>

126

Schema 30. Oxidationen mit Rutheniumtetroxid

Setzt man katalytische Mengen Rutheniumtrichlorid mit Natriummetaperiodat als Reoxidans bei $0{ }^{\circ} \mathrm{C}$ nur wenige Minuten mit Alkenen um, lassen sich je nach Substrat, in guten Ausbeuten cis-Diole gewinnen $^{[46]}$. Diese auch als Flash-Dihydroxylierung bezeichnete Me- 
thode läßt sich auch in guten Ausbeuten an elektronenarmen Alkenen wie 127 und 128 durchführen.

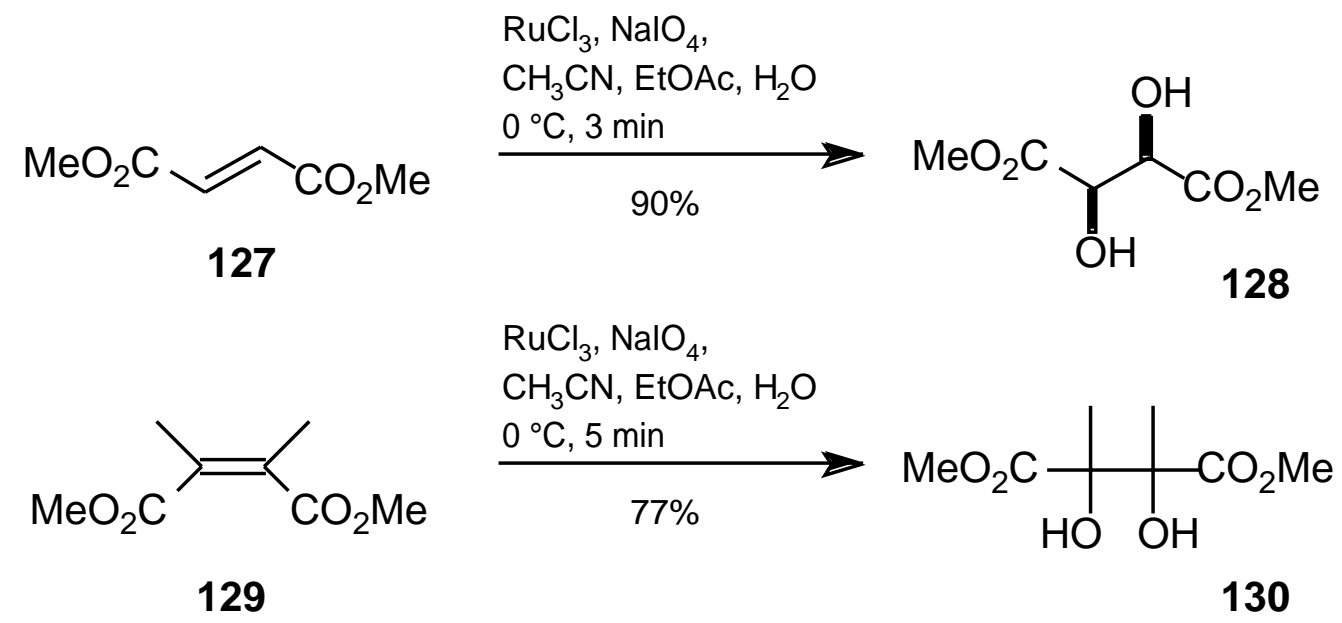

Es wurden verschiedene Endiine mit Rutheniumtetroxid umgesetzt. Die Verbindung $\mathbf{X X}$ ließ sich in guten 65\% Ausbeute in das Diol 116 überführen, aber bei anderen Endiinen lagen die Ausbeuten nur zwischen 17 und 32\% (s. Tabelle 8).

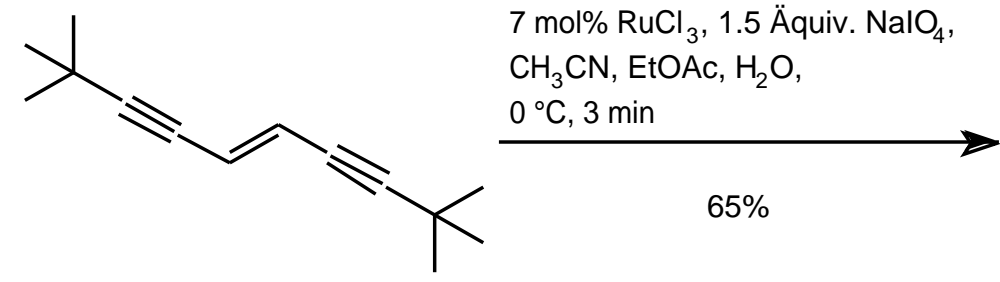

88

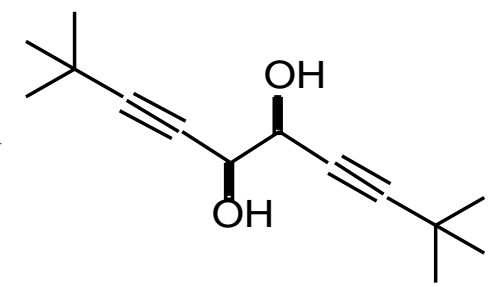

116

Es erfolgte grundsätzlich kein vollständiger Umsatz. Je länger man die Mischung rührte, desto geringer wurde der Anteil an zurückgewonnenem Edukt, ohne das sich der Produkanteil 
merklich erhöhte. Durch die Erhöhung des Rutheniumanteils von 7 auf 14mol\% konnte zwar der Umsatz erhöht werden, die Ausbeute an Produkt sank jedoch ebenfalls.

Tab. 8: Dihydroxylierungen mit Rutheniumtetroxid

\begin{tabular}{cccccc}
\hline Endiin & $\begin{array}{c}\text { Ru } \\
(\mathrm{mol} \%)\end{array}$ & $\begin{array}{c}\text { Dauer } \\
{[\mathrm{min}]}\end{array}$ & Produkt & $\begin{array}{c}\text { Edukt } \\
(\%)\end{array}$ & $\begin{array}{c}\text { Produkt } \\
(\%)\end{array}$ \\
\hline 116 & 7 & 3.5 & $\mathbf{1 3 1}$ & - & 65 \\
117 & 7 & 12 & 132 & 42 & 24 \\
119 & 7 & 3 & 133 & 53 & 26 \\
119 & 7 & 5 & 133 & 34 & 32 \\
119 & 14 & 2 & 133 & 12 & 25 \\
120 & 7 & 3 & 134 & 20 & 29 \\
\hline
\end{tabular}




\subsection{Andere Darstellungversuche für Diindiole}

Normalerweise spaltet man Alkene bei der Oxidation mit Kaliumpermanganat. Wenn man unter basischen Bedingungen arbeitet kann man die Reaktion auf der Stufe des Glykols 135 anhalten. Mit Kaliumpermanganat kann man cis-Hydroxylieren, was auch an vielen Substraten wie ungesättigten Fettsäuren erfolgreich duchgeführt wurde.

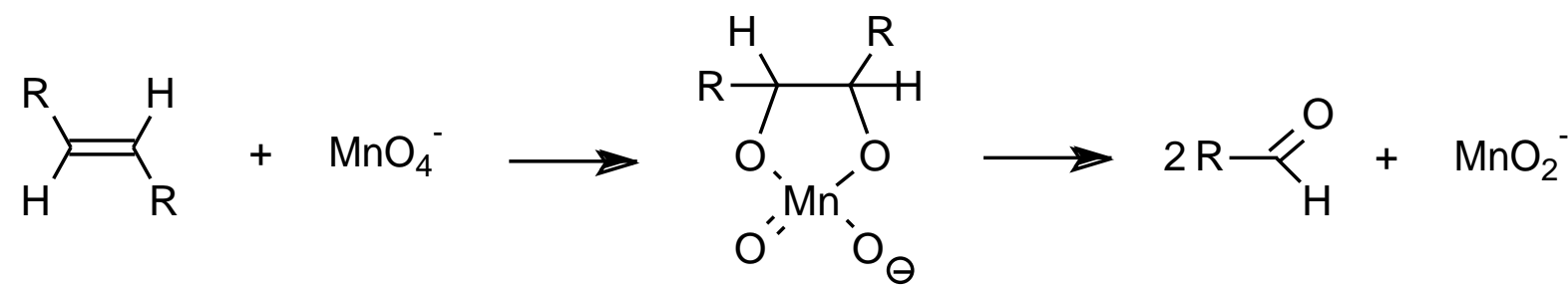

135<smiles>[Y][CH]O</smiles><smiles>[R]C(O)C(O)(O)C([R])[CH][CH+]</smiles><smiles>[R]C(O)C([R])O[Na]</smiles>

136

Schema 31. Oxidation von Alkenen mit Kaliumpermanganat

Um Alkene auch in organischen Lösungsmitteln oxidieren zu können, verwendet man Phasentransferkatalysatoren ${ }^{[4]]}$.

Umsetzungen des Endiins 98 mit Kaliumpermanganat und dem Phasentransferkatalysator Tetrabutylammoniumbromid führten zu keinem Umsatz. Auch mit Triethylbutylammoiumchlorid konnte man 98nicht zum Diol 135 umsetzen

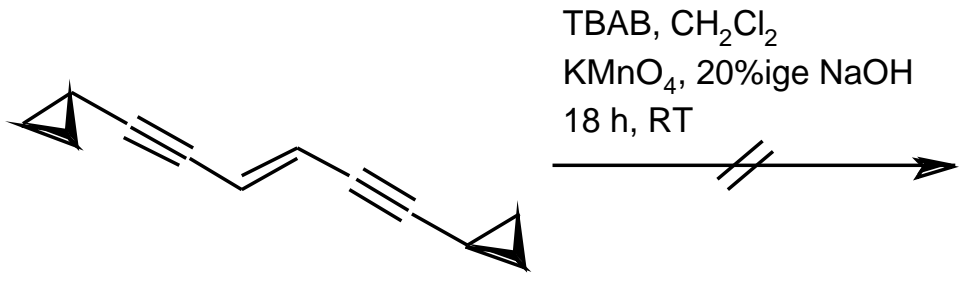

98

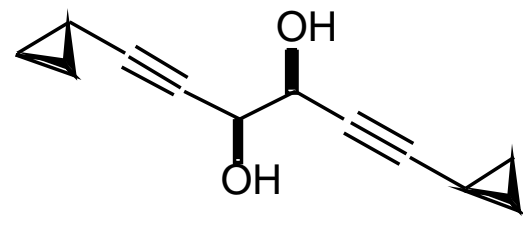

135 
Da sich die Endiine nicht sehr erfolgreich Dihydroxylieren ließen, wurde versucht die zentrale Doppelbindung zu Epoxydieren. Nach einer kürzlich von Jacobsen veröffentlichten enantioselektiven Öffnung des Epoxids mit chiralen (salen)Kobalt-Komplexen unter Zugabe von Benzoesäure, würde man einen Monobenzylester erhalten ${ }^{[48]}$. Da nach der Dihydroxylierung eine Reduktion folgt, könnte die Estergruppe in 139 gleich mit entfernt werden.

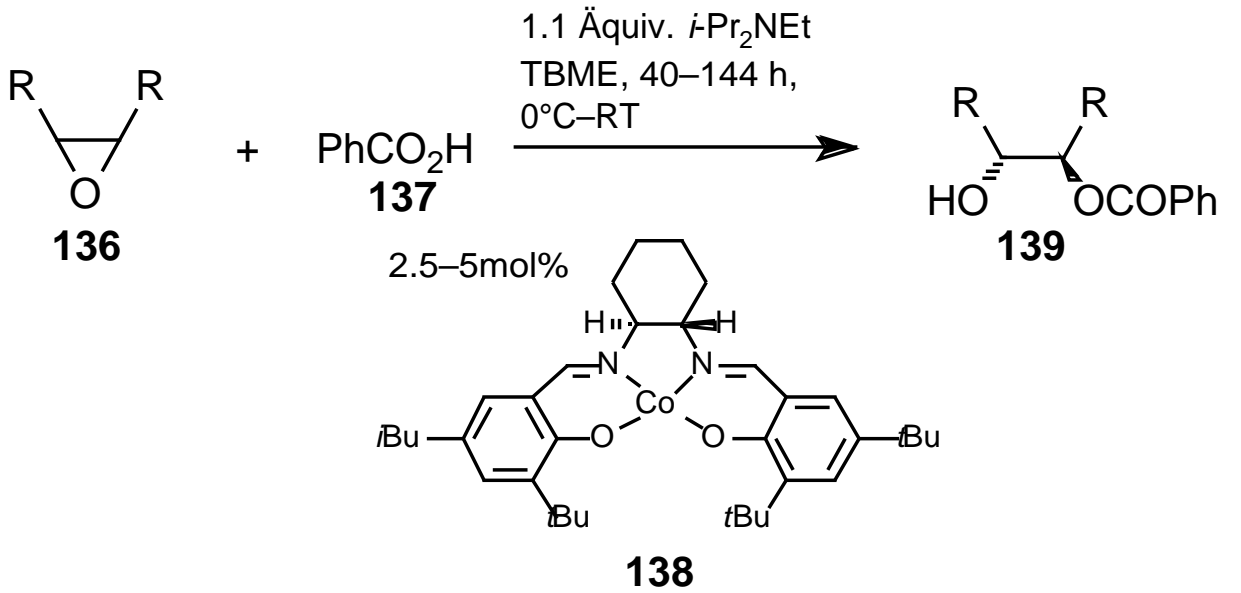

Schema 32. Enantioselektive Öffnung von Epoxiden nach Jacobsen 
Die Epoxidierung wurde an zwei Endiinen mit 3-Chlorperoxybenzoesäure durchgeführt. Es bildete sich auch bei sehr großen Überschüssen an 3-Chlorperoxybenzoesäure kein Epoxid.

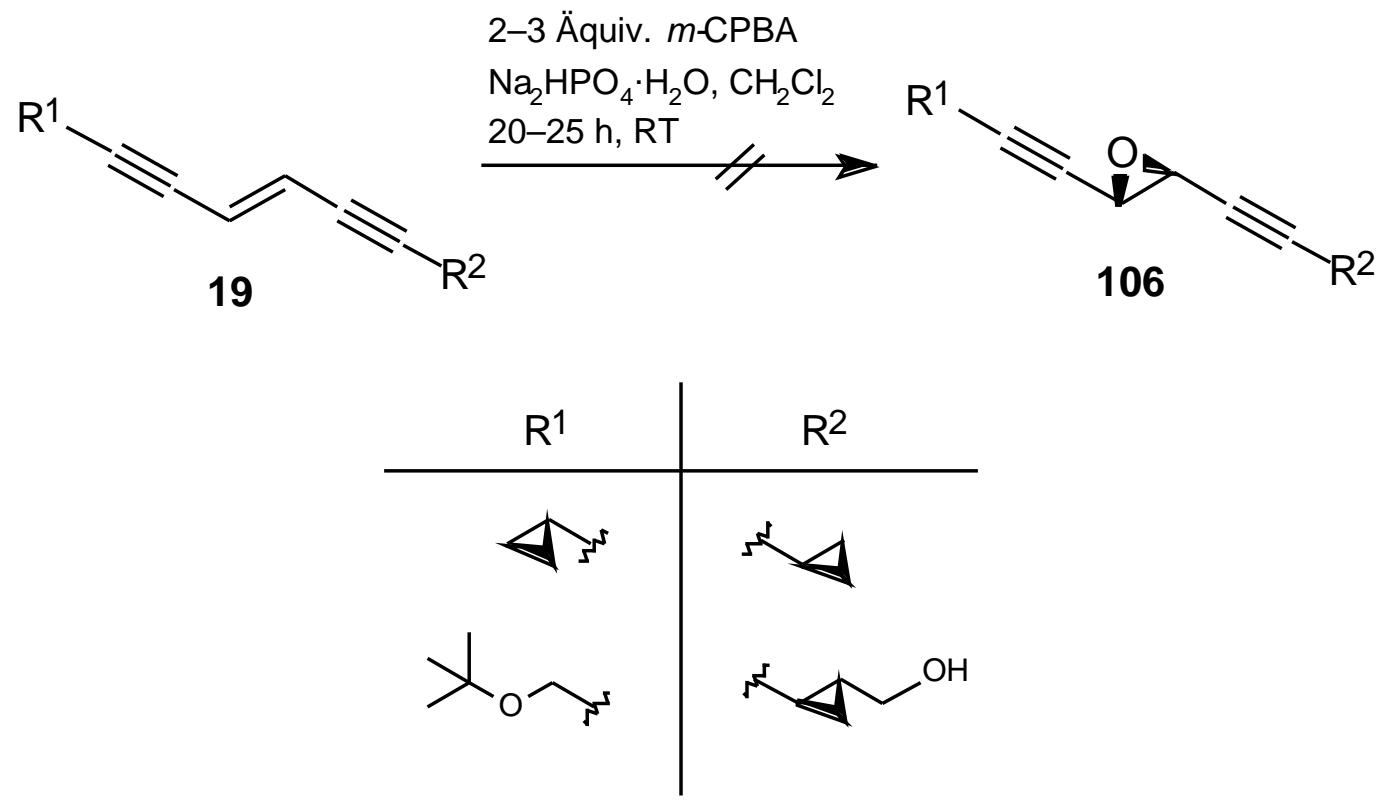

Schema 33. Versuch der Epoxidierung von Endiinen.

In der Literatur findet man wenige Darstellungsmethoden für Diindiole. Eine dieser Synthesen stützt sich auf die Dimerisierung von Ethinylcarbaldehyden ${ }^{[49]}$. Setzt man Aldehyde 139 mit Zink in Essigsäure um, erhält man symmetrische syn-Diindiole.

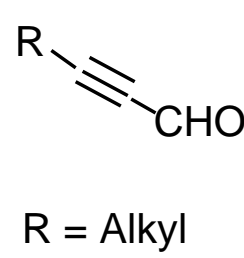

139

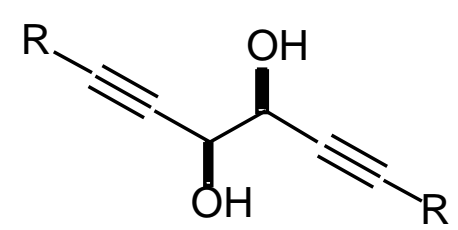

20

Schema 34. Dimerisierung von Aldehyden. 
Bei diesem Ansatz könnte man die aufwendige Darstellung der Endiine umgehen. Zuerst wurde aus tert-Butylacetylen mit $n$-Butyllithium und DMF der Aldehyd (141) dargestellt ${ }^{[50]}$ und mit Zink und Essigsäure umgesetzt. Es konnte jedoch kein Diindiol isoliert werden.

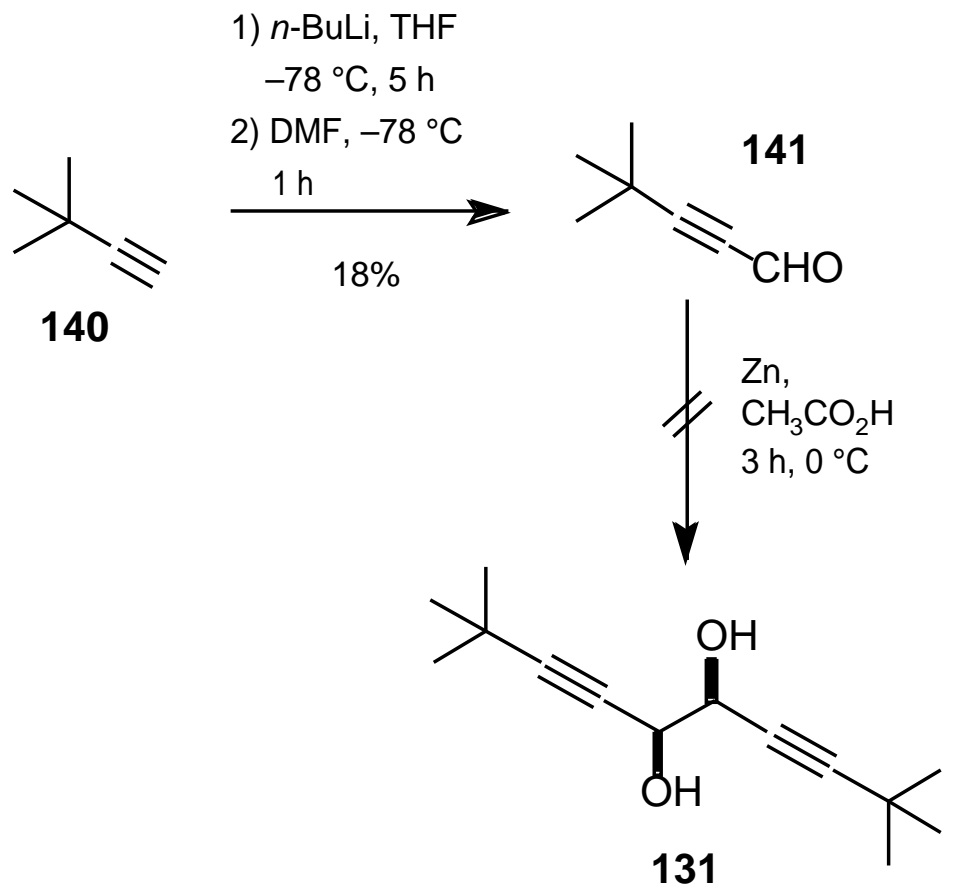




\section{Folgereaktionen der Dihydroxylierungsprodukte}

\subsection{Vorüberlegungen}

Im nächsten Schritt sollten die Diindiole 20 mit geeigneten Reduktionsmitteln zu trans-Dienen 142 umgesetzt werden. Die benachbarte Alkoholgruppe sollte diese Reduktion steuern. Über eine doppelte Cyclopropanierung an Diallylalkoholen 144 sollte diastereoselektiv 143 aufgebaut werden. Um auch die zentrale Position in ein Cyclopropan zu transformieren, sollte das Diol 143 über eine Corey-Winter-Eliminierung zu Alkenen 144 umgesetzt werden, das dann im letzten Schritt zu Oligocyclopropanen 21 cyclopropaniert werden kann.

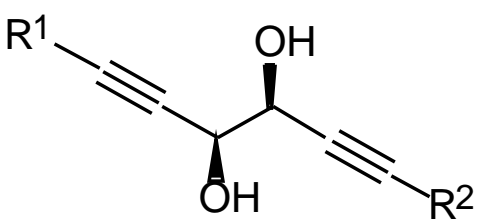

20

$\mathrm{R}^{1}$

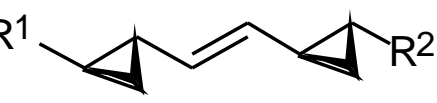

144

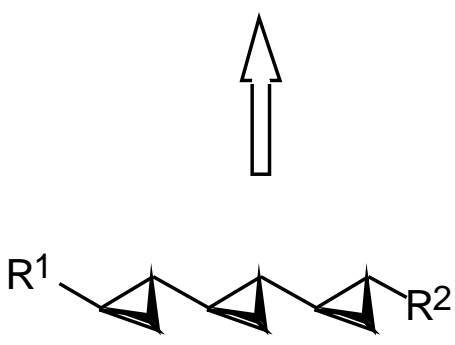

145

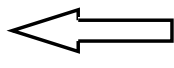<smiles>[R]C=C[C@@H](O)[C@@H](O)/C=C/[R2]</smiles>

142
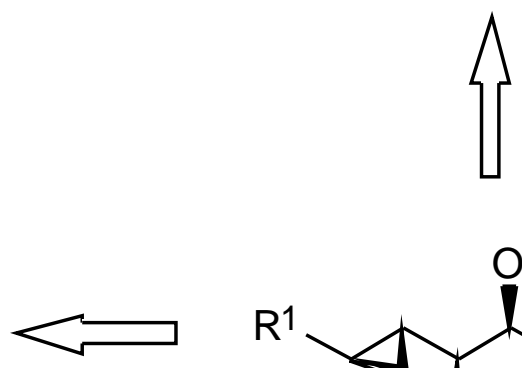<smiles>[R]C1=CC1[C@@H](O)[C@@H](O)C1C2=CC21[R2]</smiles>

143

Schema 35. Folgereaktionen an Dihydroxylierungsprodukten 


\subsection{Reduktion der Dreifachbindungen von Diindiolen}

Eine benachbarte Alkoholgruppe in Propargylalkoholen begünstigt die Reduktion zum trans-Alken. Das Verhältnis der cis/trans-Isomere hängt außerdem vom Reduktions- und Lösungsmittel ab. Als beste Kombination an 3-Trimethylsilyl-prop-2-in-1-ol stellte sich Natrium-bis(2-methoxyethoxy)aluminiumhydrid (Red-Al) und Diethylether heraus ${ }^{[51]}$.

Eine erste Reduktionen am Diindiol 131 wurde mit Lithiumaluminiumhydrid in THF durch geführt. Erst durch mehrstündiges Erhitzen unter Rückfluß konnte das Diindiol 131 zur Reaktion gebracht werden. Da befürchtet wurde, daß der durch die Reduktion enstehende Diallylalkohl 144 sehr instabil ist, wurde zuerst 131 mit Benzaldehyd als Acetal geschützt. Sowohl ungeschütztes als auch geschütztes 131 konnte in mäßigen Ausbeuten zu den gewünschten Dienen reduziert werden.

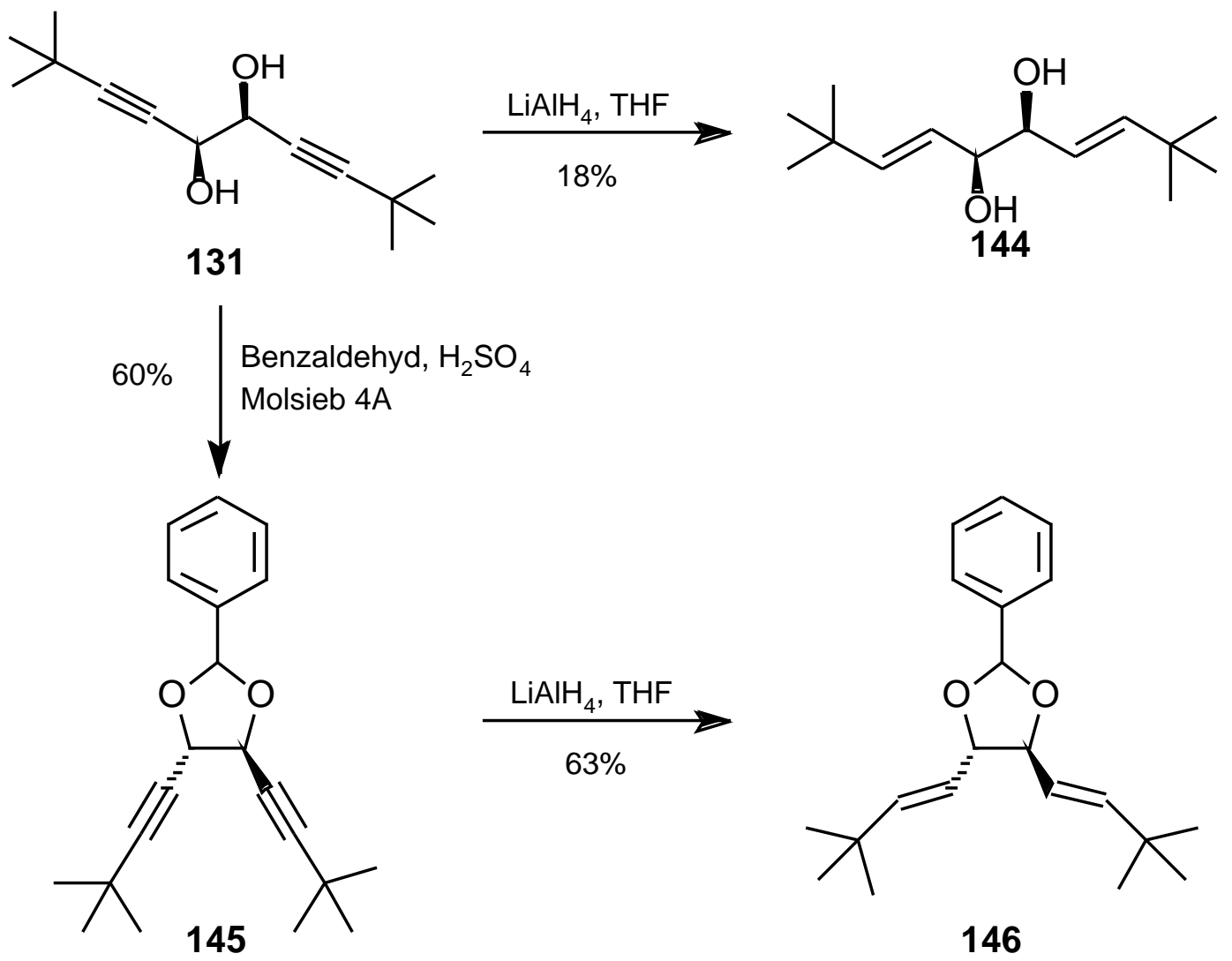

In besseren Ausbeuten konnte 119 mit Red-Al zu 147 reduziert werden. Normalerweise arbeitet man mit 1.5 Äquiv Red-Al pro zu reduzierender Doppelbindung. Um vernünftige Ergeb- 
nisse zu erzielen mußten bei der Reduktion von 1193.5 Äquivalente Red-Al pro Doppelbindung eingesetzt werden.

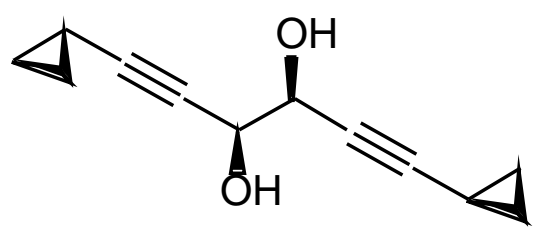

119
3.5 Äquiv. Red-Al, $\mathrm{Et}_{2} \mathrm{O}, 9 \mathrm{~h}, \mathrm{RT}$

$77 \%$

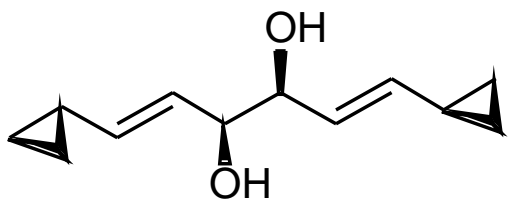

147 
4.3. Doppelte Cyclopropanierung und Corey-Winter-Eliminierung zu 1,2-Bis(bicyclopropyl)ethen

Die doppelte Cyclopropanierung von 147 gelang in guten Ausbeuten. Jedoch waren auch nach $16 \mathrm{~h}$ Reaktionsdauer bei Raumtemperatur und 5 Äquivalenten Diethylzink und 10 Äquivalenten Diiodmethan pro Doppelbindung immer noch Spuren Monocyclopropanierungsprodukt vorhanden, die säulenchromatographisch schlecht abtrennbar waren.

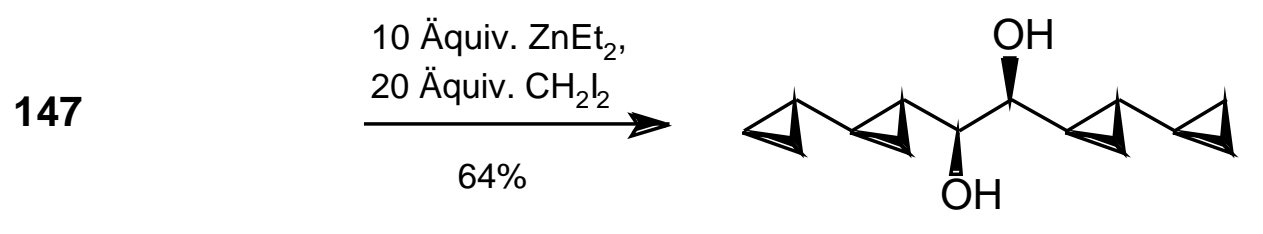

148

Diole lassen sich über die Corey-Winter-Eliminierung ${ }^{[52]}$ in Alkene umwandelt. Dabei stellt man zuerst ein cyclisches Thiocarbonat $\mathbf{1 5 1}$ her, das mit Trimethylphosphit oder anderen Phoshorverbindungen unter Erhitzen zu Alkenen reagiert. Die Reaktion läuft offensichtlich einstufig ab, wobei sich Schwefel und Phosphor verbinden und der fünfgliedrige Ring unter 
Freisetzung von Kohlendioxid zum Alken 153 reagiert. In dieser syn-Eliminierung entstehen aus syn-Diolen 149 trans-Alkene 153 und aus anti-Diolen 150 cis-Alkene 154.

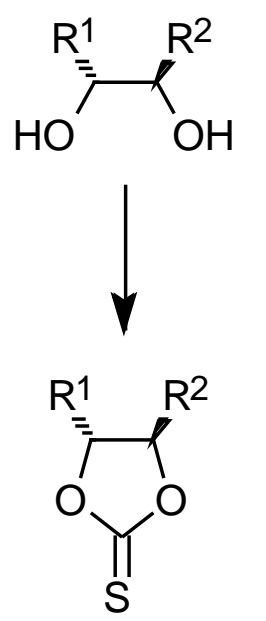

149

151

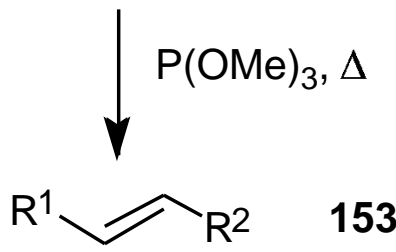

$$
+\mathrm{CO}_{2}+\mathrm{S}=\mathrm{P}(\mathrm{OMe})_{3}
$$<smiles>[R]C(O)C(O)O</smiles>

150
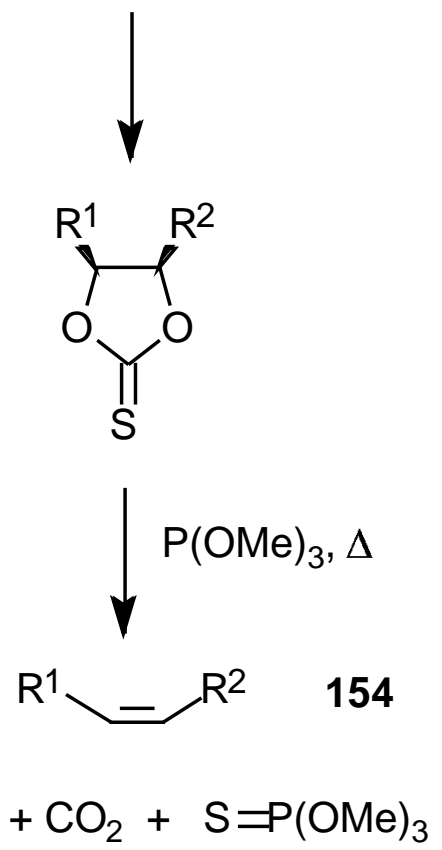

Schema 36. Mechanismus der Corey-Winter-Eliminierung

Mit Trimethylphosphit muß man die Thiocarbonate längere Zeit auf über $100{ }^{\circ} \mathrm{C}$ erwärmen, bis die Fragmentierung eintritt. Diesen Bedingungen sollten die substituierten Oligocyclopropane nicht ausgesetzt werden, deshalb wurde eine Variante der Fragmentierung mit dem Phosphan 157 gewählt, bei der die Reaktion schon bei $40{ }^{\circ} \mathrm{C}$ eintritt. 157 läßt sich leicht aus 
Dichlorphenylphosphin (155) und $N, N^{\prime}-1,2-$ Dimethylaminoethan (156) mit Triethylamin herstellen.

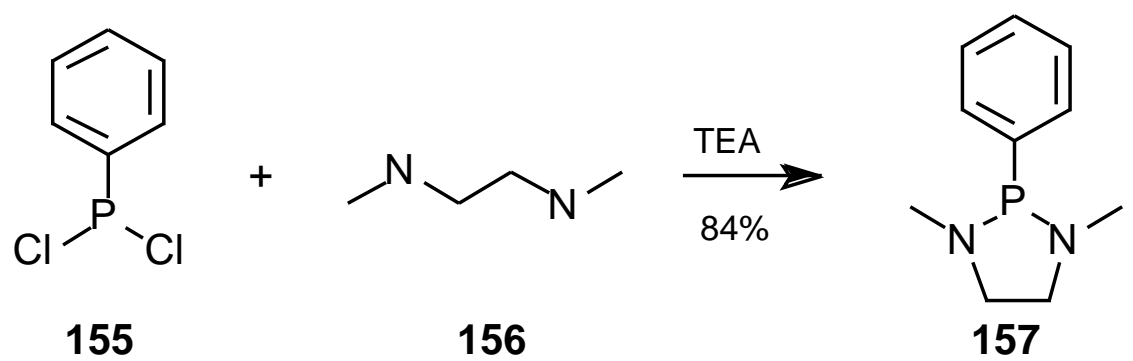

1,2-Bis(bicyclopropyl)ethan-1,2-diol (148) wurde in einer Corey-Winter Eliminierung umgesetzt. Dabei wurde das Thiocarbonat 158 ohne weitere Aufarbeitung mit 157 in 23\% Ausbeute zum Alken 159 umgesetzt.

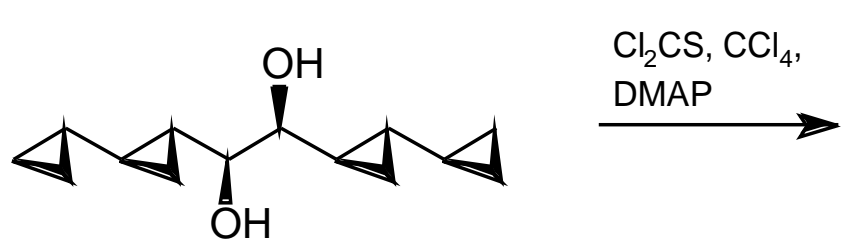

148

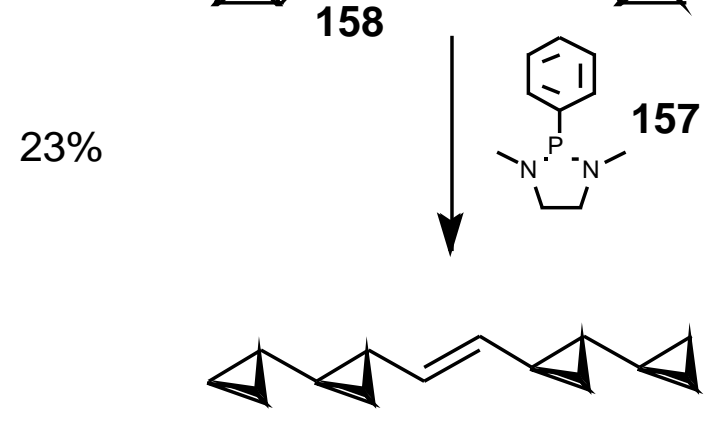

159

Im letzten Schritt müsste eine Cyclopropanierung der zentralen Doppelbindung durchgeführt werden, die wegen zu geringer Substanzmenge an 159 nicht mehr durchgeführt werden konnte. Dabei würde jedoch unselektiv die Cyclopropanierung der Doppelbindung von beiden Seiten erfolgen und ein Gemisch beider Diastereomere entstehen. Die cyclopropanierung könnte auch wieder nach der Zink-Carbenoid-Methode erfolgen. 


\section{Experimenteller Teil}

\section{Allgemeines}

Schmelzpunkte: Schmelzpunktapparatur der Fa. Büchi; die gemessenen Schmelzpunkte sind unkorrigiert. - IR: Bruker IFS 66 (FT-IR), Perkin-Elmer 298. - ${ }^{1}$ H-NMR: Bruker AM 250 (250 MHz), Varian VXR 200 (200 MHz); ( $\delta$ = 0 für Tetramethylsilan, 7.15 für [D5]Benzol, 7.26 für Chloroform. Charakterisierung der Signalaufspaltung: $\mathrm{s}=$ Singulett, br. $\mathrm{s}=$ breites Singulett, $\mathrm{d}=$ Dublett, $\mathrm{t}=$ Triplett, $\mathrm{q}=$ Quartett, $\mathrm{m}=$ Multiplett, $\mathrm{dd}=$ doppeltes Dublett, ddd $=$ doppeltes Dublett eines Dubletts, $\mathrm{dq}=$ Dublett eines Quartetts, $\mathrm{AB}=$ Signalaufspaltung entspricht einem AB-Spektrum. Spektren wurden nach erster Ordnung ausgewertet; die entsprechenden Kopplungskonstanten sind als Betrag angegeben. Abkürzungen für die Zuordnung der Signale: $\mathrm{Ph}=$ phenylisch, o-, m-, $\mathrm{p}-\mathrm{Ph}=$ ortho-, meta-, para-phenylisch, cPr = cyclopropylisch. $-{ }^{13} \mathrm{C}-\mathrm{NMR}$ : Bruker AM 250 (62.9 MHz), Varian VXR 200 (50.3 MHz); ( $\delta$ = 77.0 für Deuterochloroform, 128.0 für [D6]Benzol. Die Multiplizität der Signale wurde durch die DEPT- $($ DEPT = distortionless enhancement by polarisation transfer) Aufnahmetechnik bestimmt und ist wie folgt angegeben: DEPT: + = primäre oder tertiäre (positives DEPT-Signal), $-=$ sekundäre (negatives DEPT-Signal), $\mathrm{C}_{\text {quart }}=$ quartäre C-Atome (DEPT-Signalintensität Null). Abkürzungen für die Zuordnung der Signale: $\mathrm{Ph}=$ phenylisch, $\mathrm{o}-, \mathrm{m}-, \mathrm{p}-\mathrm{Ph}=$ ortho-, meta-, para-phenylisch, $\mathrm{cPr}=$ cyclopropylisch, $\mathrm{Bn}=$ benzylisch . Bei nicht eindeutiger Zuordnung werden jeweils alle möglichen Kohlenstoffatome angegeben.-MS: Varian MAT CH 7, MAT 731.-HRMS (Hochauflösende MS): Varian MAT 311 A; korrekte HMS = vorgewählter Ionen-Peak stimmt bei R $<10000$ mit einer Fehlerbreite von \pm 2 ppm mit der exakten Masse überein. - Optische Drehungen: Polarimeter Perkin-Elmer 243. - Säulenchromatographie (SC): Chromatographische Trennungen erfolgten an Merck Kieselgel 60 (0.063-0.200 mm, 230-400 mesh) als Normalbzw. an Macherey-Nagel Kieselgel 60 (0.04-0.063 mm, 200-400 mesh) als Flash-Chromatographie nach Still. Die Säulendimensionen wurden als "Säulendurchmesser $\times$ Höhe" der Kieselgelsäule. Alle Laufmittel wurden destilliert verwendet. - Dünnschichtchromatographie (DC): Macherey-Nagel Fertigfolien: Alugram Sil G/UV 254 ; Merck DC-Fertigfolien: Kieselgel $60 \mathrm{~F}_{254}$ auf Aluminiumfolie. Detektion unter UV-Licht bei $254 \mathrm{~nm}$, Entwicklung mit Molybdatophosphorsäure-Tauchreagenz (10proz. Lsg. in Ethanol) oder Bromdampf. Elementaranalysen: Mikroanalytisches Laboratorium des Institutes für Organische Chemie 
der Universität Göttingen. - Wasserfreie Lösungsmittel wurden nach üblichen Laboratoriumsmethoden gewonnen. Alle Umsetzungen mit Übergangsmetallkomplexen und Metallalkylen wurden, soweit nicht anders angegeben, unter Sauerstoff- und Feuchtigkeitsausschluß durchgeführt. - Benutzte Abkürzungen: EtOAc = Essigsäureethylester, $\mathrm{PE}=$ Petrolether (Spezialbenzin, Siedebereich: $40-80^{\circ} \mathrm{C}$ ), $\mathrm{DE}=$ Diethylether, $\mathrm{THF}=$ Tetrahydrofuran, $\mathrm{DMF}=$ Dimethylformamid, $\mathrm{DME}=$ Dimethoxyethan, $\mathrm{DMSO}=$ Dimethylsulfoxid. 


\section{Darstellung der Verbindungen}

\subsection{Verbindungen ausgehend vom Cyclopropran-1,2-dicarbonsäureethylmethylester}

Cyclopropandicarbonsäure-1-ethyl-2-methylester (33): $\mathrm{Zu}$ einem Gemisch von $50 \mathrm{ml}$ wasserfreiem Toluol und $21.0 \mathrm{~g}$ (0.525 mol) Natriumhydrid (60\%ige Dispersion in Mineralöl) wurde bei $60{ }^{\circ} \mathrm{C}$ eine Mischung aus $45.0 \mathrm{ml}$ (0.500 mol) Acryl-

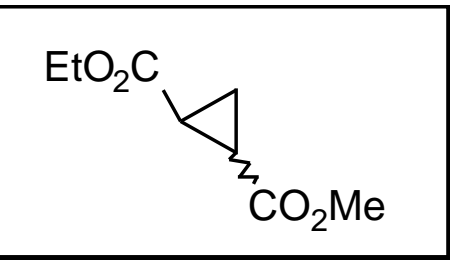
säuremethylester und $61.3 \mathrm{~g}$ (0.500 mol) Chloressigsäureethylester getropft. Die Mischung wurde 45 min bei $100{ }^{\circ} \mathrm{C}$ gehalten und dann $3.5 \mathrm{~h}$ bei Raumtemp. gerührt. Man versetzte mit $2 \mathrm{ml}$ Methanol und anschließend mit $200 \mathrm{ml}$ Wasser, extrahierte mit Diethylether $(2 \times 50 \mathrm{ml})$. Die vereinigten organischen Extrakte wurden über Magnesiumsulfat getrocknet und i. Vak. eingeengt. Fraktionierende Destillation ergab 57.5 g (67\%) 33 als cis/trans-Isomerengemisch im Verhältnis 1:1 in Form eines farblosen Öls. $-{ }^{1} \mathrm{H}-\mathrm{NMR}(250 \mathrm{MHz}$, $\left.\mathrm{CDCl}_{3}\right): \delta=1.12-1.26\left(\mathrm{~m}, 3 \mathrm{H}, \mathrm{CH}_{2} \mathrm{CH}_{3}, \mathrm{cPr}-\mathrm{H}\right), 1.27-1.43(\mathrm{~m}, 1 \mathrm{H}, \mathrm{cPr}-\mathrm{H}), 1.53-1.65(\mathrm{~m}$, $1 \mathrm{H}, \mathrm{cPr}-\mathrm{H}), 1.92-1.23(\mathrm{~m}, 2 \mathrm{H}, \mathrm{cPr}-\mathrm{H}), 3.56-3.72$ (m, 3 H, $\left.\mathrm{OCH}_{3}\right), 4.00-4.19$ (m, $2 \mathrm{H}$, $\mathrm{CH}_{2} \mathrm{CH}_{3}$ ).

trans-2-Hydroxymethylcyclopropan-1-carbonsäureethylester (34-Et) und trans-2-Hydroxymethylcyclopropan-1-carbonsäuremethylester (34-Me): Zu einer Lösung von $35.1 \mathrm{~g}$ (200 mmol) 33 in $300 \mathrm{ml}$ wasserfreiem THF wurde unter $\mathrm{N}_{2}$-Atmosphäre bei $-70^{\circ} \mathrm{C}$ innerhalb von $3 \mathrm{~h}$ portionsweise $4.1 \mathrm{~g}$ (108 mmol) Lithiumaluminiumhydrid gegeben. Man ließ die Mischung auf $0{ }^{\circ} \mathrm{C}$ erwärmen und rührte weitere $30 \mathrm{~min}$. Anschließend tropfte man langsam $50 \mathrm{ml}$ gesättigte
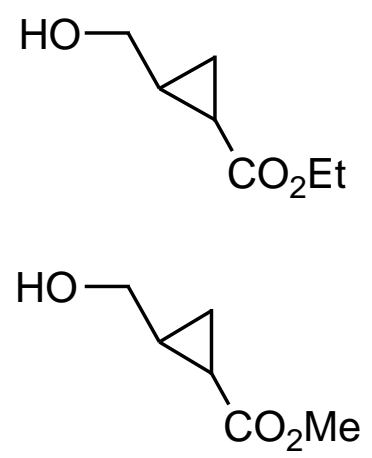

Natriumhydrogencarbonat zu. Der Niederschlag wurde abfiltriert und mit Diethylether $(500 \mathrm{ml})$ und Essigsäureethylester $(300 \mathrm{ml})$ gewaschen. Die organische Phase wurde mit gesättigter Natriumchloridlösung $(50 \mathrm{ml})$ gewaschen, über Magnesiumsulfat getrocknet und i. Vak. eingeengt. Fraktionierende Destillation ergab 25.7 g 34-Et und 34-Me als untrennbares Gemisch im Verhältnis 1:1. - ${ }^{1} \mathrm{H}-\mathrm{NMR}\left(250 \mathrm{MHz}, \mathrm{CDCl}_{3}\right): \delta=0.88-0.93(\mathrm{~m}, 2 \mathrm{H}, \mathrm{cPr}-$ $\mathrm{H}), 1.09-1.13(\mathrm{~m}, 2 \mathrm{H}, \mathrm{cPr}-\mathrm{H}), 1.20\left(\mathrm{t},{ }^{3} \mathrm{~J}=7 \mathrm{~Hz}, 3 \mathrm{H}, \mathrm{CH}_{2} \mathrm{CH}_{3}\right), 1.45-1.57$ (m, $\left.2 \mathrm{H}, \mathrm{cPr}-\mathrm{H}\right)$, 
1.45-1.67 (m, 2 H, cPr-H), 2.98 (br. s, $2 \mathrm{H}, \mathrm{OH}), 3.32-3.59$ (m, 4 H, $\mathrm{CH}_{2} \mathrm{O}$ ), 3.61 (s, $3 \mathrm{H}$, $\left.\mathrm{OCH}_{3}\right), 4.05$ (q, $\left.{ }^{3} \mathrm{~J}=7 \mathrm{~Hz}, 2 \mathrm{H}, \mathrm{CH}_{2} \mathrm{CH}_{3}\right) .-{ }^{13} \mathrm{C}-\mathrm{NMR}\left(62.9 \mathrm{MHz}, \mathrm{CDCl}_{3}\right.$, zusätzl. DEPT): $\delta=12.5$ (+, cPr-C), 12.6 (-, $\left.\mathrm{CH}_{2} \mathrm{CH}_{3}\right), 18.0$ (-, cPr-C), 18.2 (-, cPr-C), 24.1 (-, cPr-C), 24.2 (-, cPr-C), $51.7\left(-, \mathrm{OCH}_{3}\right), 60.5\left(+, \mathrm{CH}_{2} \mathrm{CH}_{3}\right), 64.0\left(+, \mathrm{HOCH}_{2}\right), 64.1\left(+, \mathrm{HOCH}_{2}\right), 174.0$ $\left(\mathrm{C}_{\text {quart }}, \mathrm{CO}_{2}\right), 174.4\left(\mathrm{C}_{\text {quart }}, \mathrm{CO}_{2}\right)$.

Versuch zur Darstellung von trans-2-tert-Butyloxymethylcyclopropan-1-carbonsäuremethylester (38): In eine Pyrexflasche wurden bei $-78{ }^{\circ} \mathrm{C} 2.81 \mathrm{~g}(50.1 \mathrm{mmol}) 2-$ Methylpropen einkondensiert, $3.30 \mathrm{~g}$ (25.0 mmol) 34-Me zugegeben und $1 \mathrm{ml} \mathrm{konz.} \mathrm{Salzsäure.} \mathrm{Es} \mathrm{wurde} 20 \mathrm{~h}$ bei Raumtemp. gerührt, auf $-78{ }^{\circ} \mathrm{C}$ gekühlt und $10 \mathrm{ml}$ gesättigte Natriumhydrogencarbonatlösung zugetropft. Man extrahierte mit Diethylether $(3 \times 15 \mathrm{ml})$, trocknete über Magnesiumsulfat und entfernte das Lösungsmittel i. Vak. Es konnten nur $2.39 \mathrm{~g}$ (90\%) 34-Me zurückgewonnen werden.

trans-2-Benzyloxymethylcyclopropan-1-carbonsäuremethylester (36-Me) und trans-2-Benzyloxymethylcyclopropan-1carbonsäureethylester (36-Et): Zu einer Mischung von $13.3 \mathrm{~g}$ (333 mmol) Natriumhydrid (60\%ige Dispersion in Mineralöl) wurde bei $0{ }^{\circ} \mathrm{C}$ eine Lösung von $41.5 \mathrm{~g}$ 34-Me und 34-Et als 1:1 Gemisch in $50 \mathrm{ml}$ THF zugetropft. Man ließ auf Raumtemp. erwärmen und rührte $1 \mathrm{~h}$ weiter. Dann wurden $38.1 \mathrm{ml}$ (320 mmol) Benzylbromid zugetropft und $20 \mathrm{~h}$ bei Raum-
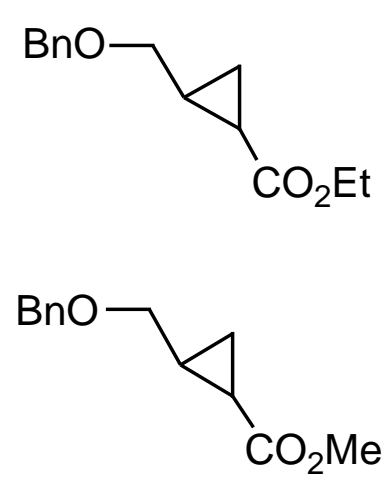
temp. gerührt. Die Mischung wurde in $300 \mathrm{ml}$ Wasser gegeben und mit Diethylether $(3 \times 200 \mathrm{ml})$ extrahiert. Die Extrakte wurden über Magnesiumsulfat getrocknet und i. Vak. eingeengt. Fraktionierende Destillation ergab 38.1 g (Sdp. 120-125 ${ }^{\circ} \mathrm{C} / 0.07$ Torr) 36-Me und 36-Et als 1:1 Gemisch. - ${ }^{1} \mathrm{H}-\mathrm{NMR}\left(250 \mathrm{MHz}, \mathrm{CDCl}_{3}\right): \delta=0.83-0.95$ (m, $\left.4 \mathrm{H}, \mathrm{cPr}-\mathrm{H}\right)$, 1.18-1.36 (m, $\left.5 \mathrm{H}, \mathrm{CH}_{2} \mathrm{CH}_{3}, \mathrm{cPr}-\mathrm{H}\right), 1.53-1.63$ (m, 2 H, cPr-H), 1.66-1.82 (m, 2 H, cPr-H), 3.27-3.45 (m, $\left.4 \mathrm{H}, \mathrm{CH}_{2}\right), 3.64\left(\mathrm{~s}, 3 \mathrm{H}, \mathrm{OCH}_{3}\right), 4.12\left(\mathrm{q},{ }^{3} \mathrm{~J}=4 \mathrm{~Hz}, 2 \mathrm{H}, \mathrm{CH}_{2} \mathrm{CH}_{3}\right), 4.53(\mathrm{~s}$, $\left.4 \mathrm{H}, \mathrm{PhCH}_{2}\right), 7.23-7.40$ (m, $\left.10 \mathrm{H}, \mathrm{Ph}-\mathrm{H}\right) .-{ }^{13} \mathrm{C}-\mathrm{NMR}\left(62.9 \mathrm{MHz}, \mathrm{CDCl}_{3}\right.$, zusätzl. DEPT): 
$\delta=12.9$ (-, cPr-C), 14.1 (+, $\left.\mathrm{CH}_{2} \mathrm{CH}_{3}\right), 18.3$ (+, cPr-C), 18.4 (+, cPr-C), 21.5 (+, cPr-C), 21.6 (cPr-C), $51.7\left(+, \mathrm{OCH}_{3}\right), 60.4\left(-, \mathrm{CH}_{2} \mathrm{CH}_{3}\right), 71.3\left(-, \mathrm{CH}_{2}\right), 71.4\left(-, \mathrm{CH}_{2}\right), 72.5\left(-, \mathrm{PhCH}_{2}\right)$, 127.5 (+, Ph-C), 128.3 (+. Ph-C), $138.0\left(\mathrm{C}_{\text {quart }}, \mathrm{Ph}-\mathrm{C}\right), 173.7\left(\mathrm{C}_{\text {quart }}, \mathrm{CO}_{2}\right), 174.1\left(\mathrm{C}_{\text {quart }}, \mathrm{CO}_{2}\right)$.

trans-1-Benzyloxymethyl-2-hydroxymethylcyclopropan (39):

$\mathrm{Zu}$ einer Lösung von $13.3 \mathrm{~g}$ (350 mmol) Lithiumaluminiumhydrid in $300 \mathrm{ml}$ wasserfreiem THF wurden bei $0{ }^{\circ} \mathrm{C} 38.1 \mathrm{~g}$ (173 mmol) eines 1:1 Gemisches von 36-Me und 36-Et in $50 \mathrm{ml}$ THF getropft. Man ließ die Reaktionsmischung auf

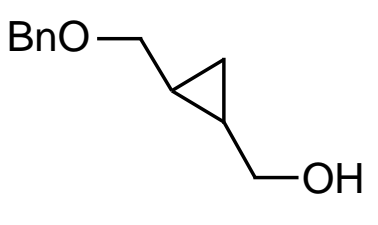

Raumtemp. erwärmen und rührte $12 \mathrm{~h}$ weiter. Die Mischung wurde vorsichtig mit gesättigter Magnesiumsulfatlösung $(75 \mathrm{ml})$ hydrolysiert, der Niederschlag abfiltriert und mit Essigester (700 ml) gewaschen. Die vereinigten organischen Phasen wurden mit gesättigter Natriumchloridlösung gewaschen $(2 \times 50 \mathrm{ml})$, über Magnesiumsulfat getrocknet und i. Vak. eingeengt. Fraktionierende Destillation ergab 30.2 g (91\%) 39 (Sdp. 110-115 C/0.07 Torr) als farbloses Öl. - IR (Film): v = $3481 \mathrm{~cm}^{-1}, 3089\left(\mathrm{CH}_{2}\right), 3021,2948,2851,1420,1322,1084$, 743 (C-H-Aromat), 694 (C-H-Aromat). $-{ }^{1} \mathrm{H}-\mathrm{NMR}\left(250 \mathrm{MHz}, \mathrm{CDCl}_{3}\right): \delta=0.41-52(\mathrm{~m}$, $2 \mathrm{H}, \mathrm{cPr}-\mathrm{H}), 0.93-1.05$ (m, $2 \mathrm{H}, \mathrm{cPr}-\mathrm{H}), 2.88$ (br. s, $1 \mathrm{H}, \mathrm{OH}), 3.20-3.38$ (m, $2 \mathrm{H}, \mathrm{CH}_{2} \mathrm{OH}$ ), 3.39-3.55 (m, $\left.2 \mathrm{H}, \mathrm{OCH}_{2}\right), 4.52$ (s, $\left.2 \mathrm{H}, \mathrm{PhCH}_{2}\right), 7.10-7.38$ (m, $\left.5 \mathrm{H}, \mathrm{Ph}-\mathrm{H}\right) .-{ }^{13} \mathrm{C}-\mathrm{NMR}$ (62.9 $\mathrm{MHz}^{\mathrm{CDCl}} \mathrm{CD}_{3}$, zusätzl. DEPT): $\delta=7.9$ (+, cPr-C), 16.8 (-, cPr-C), 19.8 (-, cPr-C), 66.1 $\left(+, \mathrm{CH}_{2}\right), 72.6\left(+, \mathrm{CH}_{2}\right), 73.6\left(+, \mathrm{CH}_{2}\right), 127.7$ (-, Ph-C), 127.8 (-, Ph-C), 128.4 (-, Ph-C) $138.2\left(\mathrm{C}_{\text {quart }}, \mathrm{Ph}-\mathrm{C}\right) .-\mathrm{MS}(70 \mathrm{eV}), \mathrm{m} / z(\%): 192(1)\left[\mathrm{M}^{+}\right], 107$ (41) $\left[\mathrm{OCH}_{2} \mathrm{Ph}^{+}\right], 91$ (100) $\left[\mathrm{CH}_{2} \mathrm{Ph}^{+}\right]$.

trans-2-Benzyloxymethylcyclopropan-1-carbaldehyd (48): $\mathrm{Zu}$ einer Lösung von $9.00 \mathrm{ml}$ (103 mmol) Oxalsäuredichlorid in $200 \mathrm{ml}$ wasserfreiem Dichlormethan wurden bei $-70{ }^{\circ} \mathrm{C} 15.3 \mathrm{ml}$ (216 mmol) DMSO zugetropft und 10 min bei dieser Temperatur gerührt. Danach wurde eine Lösung von $18.3 \mathrm{~g}(95.0 \mathrm{mmol})$

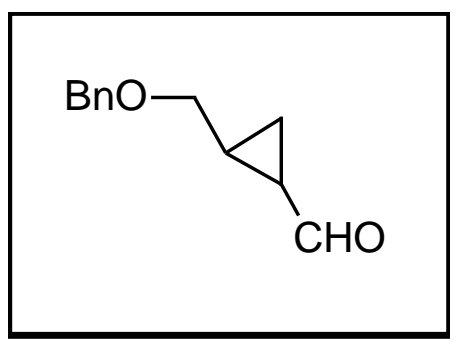
39 in $80 \mathrm{ml}$ Dichlormethan zugetropft und $30 \mathrm{~min}$ bei $-70^{\circ} \mathrm{C}$ weitergerührt. Man tropfte dann $63.0 \mathrm{ml}$ (452 mmol) Triethylamin zu, ließ auf Raumtemp. erwärmen und extrahierte 
mit Dichlormethan (300 ml). Die vereinigten organischen Extrakte wurden mit gesättigter Natriumchlordlösung gewaschen, über Magnesiumsulfat getrocknet und das Lösungsmittel i. Vak. entfernt. Säulenfiltration über Kieselgel $[3.0 \times 30 \mathrm{~cm}$, Eluens Pentan/Diethylether (1:1), $\left.R_{\mathrm{f}}=0.47\right]$ erhielt man $15.5 \mathrm{~g}(85 \%) 48$ in Form eines farblosen Öls. - IR (Film): $v=3480 \mathrm{~cm}^{-1}, 3089\left(\mathrm{CH}_{2}\right), 3021,2950,2852,1420,1322,1085,743$ (C-H-Aromat), 694 (C-H-Aromat). $-{ }^{1} \mathrm{H}-\mathrm{NMR}\left(250 \mathrm{MHz}, \mathrm{CDCl}_{3}\right): \delta=1.04-1.12$ (m, $\left.1 \mathrm{H}, \mathrm{cPr}-\mathrm{H}\right), 1.25-1.38$ (m, $1 \mathrm{H}, \mathrm{cPr}-\mathrm{H}), 1.76-1.88(\mathrm{~m}, 2 \mathrm{H}, \mathrm{cPr}-\mathrm{H}), 3.37-3.52\left(\mathrm{~m}, 2 \mathrm{H}, \mathrm{OCH}_{2}\right), 4.52(\mathrm{~s}, 2 \mathrm{H}$, $\mathrm{PhCH}_{2}$ ), 7.21-7.40 (m, $\left.5 \mathrm{H}, \mathrm{Ph}-\mathrm{H}\right), 9.11$ (d, $\left.{ }^{3} \mathrm{~J}=4 \mathrm{~Hz}, 1 \mathrm{H}, \mathrm{CHO}\right) .-{ }^{13} \mathrm{C}-\mathrm{NMR}(62.9 \mathrm{MHz}$, $\mathrm{CDCl}_{3}$, zusätzl. DEPT): $\delta=12.4$ (-, cPr-C), 21.5 (+, cPr-C), 28.0 (+, cPr-C), 70.9 (-, $\mathrm{CH}_{2}$ ), $72.4\left(-, \mathrm{CH}_{2}\right), 127.6$ (+, Ph-C), 127.7 (+, Ph-C), 128.4 (+, Ph-C), $137.9\left(\mathrm{C}_{\text {quart }}, \mathrm{Ph}-\mathrm{C}\right), 200.3$ (+, CHO). - MS (70 eV), m/z (\%): $192(1)\left[\mathrm{M}^{+}\right], 107$ (41) $\left[\mathrm{OCH}_{2} \mathrm{Ph}^{+}\right], 91$ (100) $\left[\mathrm{CH}_{2} \mathrm{Ph}^{+}\right] .-$ $\mathrm{C}_{12} \mathrm{H}_{14} \mathrm{O}_{2}$ (190.24): ber. C 75.76, H 7.42, gef. C 75.66, H 7.44.

trans-2-Benzyloxymethyl-1-ethinylcyclopropan (53): Eine Lösung von $13.1 \mathrm{~g}$ (200 mmol) Zinkpulver, 52.4 g (200 mmol) Triphenylphosphan und $66.4 \mathrm{~g}(200 \mathrm{mmol})$ Tetrabrommethan in $600 \mathrm{ml}$ wasserfreiem Dichlormethan wurden $26 \mathrm{~h}$ bei Raumtemp. gerührt. Die Reaktionsmischung wurde auf $0{ }^{\circ} \mathrm{C}$ gekühlt und eine

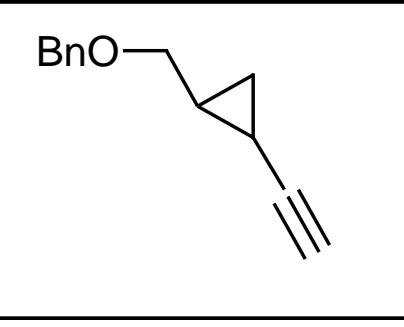
Lösung von $21.1 \mathrm{~g}$ (110 mmol) 48 in $50 \mathrm{ml}$ Dichlormethan zugetropft. Man rührte das Gemisch 2 h bei Raumtemp., gab $300 \mathrm{ml}$ Pentan dazu und ließ den Niederschlag absitzen. Es wurde dekantiert, über Magnesiumsulfat getrocknet und das Lösungsmittel i. Vak. entfernt. Der Rückstand wurde in $600 \mathrm{ml}$ wasserfreiem THF gelöst, auf $-78{ }^{\circ} \mathrm{C}$ gekühlt und $93.2 \mathrm{ml}$ (220 mmol) einer 2.36 M Lösung von n-Butyllithium in Hexan zugetropft. Man rührte die Mischung $1 \mathrm{~h}$ bei $-78^{\circ} \mathrm{C}$, ließ auf Raumtemp. erwärmen und hydrolysierte durch Zugabe von $200 \mathrm{ml}$ Wasser. Es wurde mit Diethylether extrahiert $(2 \times 100 \mathrm{ml})$, die organischen Phasen mit gesättigter Natriumchloridlösung gewaschen, über Magnesiumsulfat getrocknet und das Lösungsmittel i. Vak. entfernt. Fraktionierende Destillation ergab 14.5 g (71\%) 53 (Sdp. $80-85^{\circ} \mathrm{C} / 0.07$ Torr) als farblose Flüssigkeit. - IR (Film): $v=3293 \mathrm{~cm}^{-1}, 3029,2858,2116$, 1496, 1454, 1359, 1203, 1094, 738, 698. - ${ }^{1} \mathrm{H}-\mathrm{NMR}\left(250 \mathrm{MHz}, \mathrm{CDCl}_{3}\right): \delta=0.75-0.80(\mathrm{~m}$, $1 \mathrm{H}, \mathrm{cPr}-\mathrm{H}), 0.92-0.99$ (m, $1 \mathrm{H}, \mathrm{cPr}-\mathrm{H}), 1.15-1.27$ (m, $1 \mathrm{H}, \mathrm{cPr}-\mathrm{H}), 1.45-1.53$ (m, $1 \mathrm{H}, \mathrm{cPr}-$ H), $1.82\left(\mathrm{~d},{ }^{4} \mathrm{~J}=2 \mathrm{~Hz}, 1 \mathrm{H}, \mathrm{CH}\right), 3.36\left(\mathrm{dd},{ }^{2} \mathrm{~J}=2,{ }^{3} \mathrm{~J}=6 \mathrm{~Hz}, 2 \mathrm{H}, \mathrm{OCH}_{2}\right), 4.53(\mathrm{~s}, 2 \mathrm{H}$, 
$\mathrm{PhCH}_{2}$ ), 7.28-7.37 (m, $\left.5 \mathrm{H}, \mathrm{Ph}-\mathrm{H}\right) .-{ }^{13} \mathrm{C}-\mathrm{NMR}\left(62.9 \mathrm{MHz}, \mathrm{CDCl}_{3}\right.$, zusätzl. DEPT): $\delta=4.8$ (+, cPr-C), 13.0 (-, cPr-C), 21.4 (+, cPr-C), $64.3\left(\mathrm{C}_{\text {quart }}, \mathrm{C} \equiv \mathrm{C}\right), 72.0\left(-, \mathrm{CH}_{2}\right), 72.5\left(-, \mathrm{CH}_{2}\right)$, 86.2 (+, C $\equiv \mathrm{C}), 127.6$ (+, Ph-C), 128.4 (+, Ph-C), 138.1 ( $\left.\mathrm{C}_{\text {quart }}, \mathrm{Ph}-\mathrm{C}\right),$. - MS (DCI, $\left.70 \mathrm{eV}\right)$, $m / z(\%): 221(5)\left[\mathrm{M}+\mathrm{NH}_{3}+\mathrm{NH}_{4}^{+}\right], 206(18)\left[\mathrm{M}+\mathrm{NH}_{4}^{+}\right], 204$ (100) $\left[\mathrm{M}+\mathrm{NH}_{4}^{+}\right], 187$ (2) $\left[\mathrm{M}+\mathrm{H}^{+}\right] .-\mathrm{C}_{13} \mathrm{H}_{14} \mathrm{O}$ (186.25): ber. C 83.83, H 7.58, gef. C 82.50, H 7.75.

Versuch zur Darstellung von trans-2-Benzyloxymethyl-1-ethinylcyclopropan (53): Zu einer Lösung von 4.46 g (10.2 mmol) (Brommethyl)-triphenylphosphoniumbromid in $50 \mathrm{ml}$ wasserfreiem THF wurde bei $-78^{\circ} \mathrm{C}$ eine Lösung von $2.46 \mathrm{~g}$ (22.0 mmol) Kalium-tert-butanolat getropft. Man rührte $5 \mathrm{~min}$ bei $-78^{\circ} \mathrm{C}$ weiter und tropfte dann eine Lösung von $1.92 \mathrm{~g}$ (10.0 mmol) 48 dazu, ließ weitere $15 \mathrm{~min}$ bei $-78^{\circ} \mathrm{C}$ rühren und erwärmte im Eisbad auf $0{ }^{\circ} \mathrm{C}$. Die Reaktionsmischung wurde auf $100 \mathrm{ml}$ Eiswasser gegeben und mit Diethylether $(3 \times 50 \mathrm{ml})$ extrahiert. Die vereinigten organischen Extrakte wurden mit $20 \mathrm{ml}$ gesättigter Natriumhydrogencarbonatlösung und $20 \mathrm{ml}$ gesättigter Natriumchloridlösung gewaschen, über Magnesiumsulfat getrocknet und i. Vak. eingeengt. Es konnte kein Produkt isoliert werden.

trans-1,2-Bis(hydroxymethyl)cyclopropan (54): Zu einer Lösung von $4.20 \mathrm{~g}(110 \mathrm{mmol})$ Lithiumaluminiumhydrid in $100 \mathrm{ml}$ wasserfreiem THF wurde bei $5{ }^{\circ} \mathrm{C}$ und eine Lösung von $17.2 \mathrm{~g}$ (100 mmol) 33 in $100 \mathrm{ml}$ THF getropft. Man rührte

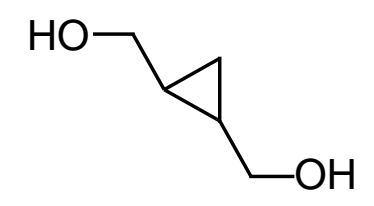
$12 \mathrm{~h}$ bei Raumtemp., kühlte die Mischung dann auf $0{ }^{\circ} \mathrm{C}$ und hydrolysierte mit $50 \mathrm{ml}$ Wasser. Der Niederschlag wurde mit konz. Salzsäure aufgelöst und die Mischung im Perforator $2.5 \mathrm{~d}$ mit Diethylether extrahiert. Die organische Phase wurde über Magnesiumsulfat getrocknet und i. Vak. eingeengt. Man erhielt 4.05 g (40\%) 54 als farbloses Öl. - IR (Film): $v=3313 \mathrm{~cm}^{-1}, 3003,2874,1653,1424,1159,1025 .-{ }^{1} \mathrm{H}-\mathrm{NMR}\left(250 \mathrm{MHz}, \mathrm{DMSO}-\mathrm{D}_{6}\right)$ : $\delta=0.23-0.42(\mathrm{~m}, 2 \mathrm{H}, \mathrm{cPr}-\mathrm{H}), 0.70-0.94(\mathrm{~m}, 2 \mathrm{H}, \mathrm{cPr}-\mathrm{H}), 3.12-3.35\left(\mathrm{~m}, 4 \mathrm{H}, \mathrm{CH}_{2}\right), 4.21$ (br. s, $2 \mathrm{H}, \mathrm{OH}) .-{ }^{13} \mathrm{C}-\mathrm{NMR}\left(62.9 \mathrm{MHz}, \mathrm{DMSO}_{6}\right.$, zusätzl. DEPT): $\delta=8.1$ (+, cPr-C), 19.3 (c-, Pr-C), 64.5 (+, $\mathrm{CH}_{2}$ ). - MS (DCI, $\left.70 \mathrm{eV}\right), \mathrm{m} / \mathrm{z}(\%): 137$ (4) $\left[\mathrm{M}^{+}+\mathrm{NH}_{3}+\mathrm{NH}_{4}^{+}\right]$, $120(100)\left[\mathrm{M}+\mathrm{NH}_{4}^{+}\right], 102(8)\left[\mathrm{M}+\mathrm{NH}_{4}{ }^{+}-\mathrm{H}_{2} \mathrm{O}\right]$. 
trans-Cyclopan-1,2-dicarbaldehyd (55): Zu einer Lösung von $4.71 \mathrm{ml}$ (54.0 mmol) Oxalsäuredichlorid in $100 \mathrm{ml}$ wasserfreiem Dichlormethan wurde bei $-70{ }^{\circ} \mathrm{C}$ eine Lösung von $7.17 \mathrm{ml}$ (108 mmol) DMSO in $30 \mathrm{ml}$ Dichlormethan zugetropft. Die

$\mathrm{OHC}$

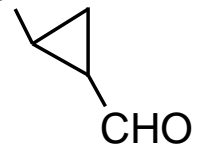

Mischung wurde 10 min bei derselben Temperatur weitergerührt und dann eine Lösung von $2.50 \mathrm{~g}$ (24.5 mmol) 54 in $40 \mathrm{ml}$ Dichlormethan zugetropft. Man rührte $30 \mathrm{~min}$ weiter und gab dann $37.6 \mathrm{ml}$ (270 mmol) Triethylamin dazu, ließ auf Raumtemp. erwärmen und extrahierte mit Dichlormethan (100 ml). Die vereinigten organischen Extrakte wurden mit gesättigter Natriumchlordlösung gewaschen, über Magnesiumsulfat getrocknet und das Lösungsmittel i. Vak. (5 Torr) unter Kühlen der Vorlage in Eiswasser entfernt. Der Rückstand wurde umkondensiert (0.07 Torr) und man erhielt $2.10 \mathrm{~g}(86 \%) 55$ als farblose Flüssigkeit. - ${ }^{1} \mathrm{H}-\mathrm{NMR}\left(250 \mathrm{MHz}, \mathrm{CDCl}_{3}\right): \delta=1.62-1.73(\mathrm{~m}, 2 \mathrm{H}, \mathrm{cPr}-\mathrm{H}), 2.25-2.37$ (m, $2 \mathrm{H}, \mathrm{cPr}-\mathrm{H}), 9.30\left(\mathrm{~d},{ }^{3} \mathrm{~J}=2 \mathrm{~Hz}, 2 \mathrm{H}, \mathrm{CHO}\right) .-{ }^{13} \mathrm{C}-\mathrm{NMR}\left(62.9 \mathrm{MHz}, \mathrm{CDCl}_{3}\right): \delta=14.2(\mathrm{cPr}-$ C), 30.1 (cPr-C), 197.0 (CHO).

\subsection{Verbindungen ausgehend vom Pent-2-en-4-in-1-ol}

Pent-2-en-4-in-1-ol (23): In einen auf $-78^{\circ} \mathrm{C}$ gekühlten Kolben wurden 1.51 Ammoniak einkondensiert, $1.00 \mathrm{~g} \mathrm{Ei-}$ sen(III)nitrat zugegeben und langsam $52.8 \mathrm{~g} \quad(2.30 \mathrm{~mol})$

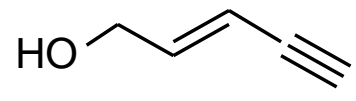

Natrium gelöst. In diese Lösung wurde $1.5 \mathrm{~h}$ Ethin eingeleitet, das vorher durch eine auf $78{ }^{\circ} \mathrm{C}$ gekühlte und eine mit konz. Schwefelsäure gefüllte Kühlfalle geleitet wurde. Innerhalb von $1.5 \mathrm{~h}$ wurde dann bei $-55^{\circ} \mathrm{C}$ Innentemperatur $78.2 \mathrm{ml}$ (1.00 mmol) Epichlorhydrin zugetropft. Die Reaktionsmischung wurde $1 \mathrm{~h}$ bei dieser Temperatur weitergerührt und 45 min bei $-33{ }^{\circ} \mathrm{C}$ unter Rückfluß gehalten. Das Ammoniak ließ man über Nacht abdampfen und hydrolysierte den Rückstand vorsichtig unter Eiskühlung mit 500 ml gesättigter Ammoniumchloridlösung. Es wurde mit Diethylether extrahiert $(7 \times 120 \mathrm{ml})$, die vereinigten organischen Extrakte über Kaliumcarbonat getrocknet und das Lösungsmittel unter vermindertem Druck entfernt. Der Rückstand wurde umkondensiert (16 Torr), mit einer Spatelspitze Hydrochinon versetzt und fraktionierend über eine $30 \mathrm{~cm}$ Vigreuxkolonne destilliert (Sdp. $70-75^{\circ} \mathrm{C} / 16$ Torr). Man erhielt 28.3 g (34\%) 23 in Form einer klaren Flüssigkeit, 
die sich bei Raumtemp. innerhalb weniger Stunden gelb färbt. - IR (Film): $v=3293 \mathrm{~cm}^{-1}$, 2919, 2865, 1448, 1369, 1266, 1091, 1039, 995, 955. - ${ }^{1} \mathrm{H}-\mathrm{NMR}\left(250 \mathrm{MHz}, \mathrm{CDCl}_{3}\right)$ : $\delta=2.36-2.53$ (br. s, $1 \mathrm{H}, \mathrm{OH}), 2.42(\mathrm{~s}, 1 \mathrm{H}, \mathrm{C} \equiv \mathrm{CH}), 4.21\left(\mathrm{~d},{ }^{3} \mathrm{~J}=3 \mathrm{~Hz}, 2 \mathrm{H}, \mathrm{CH}_{2}\right), 5.23(\mathrm{dt}$, $\left.{ }^{3} J=3,{ }^{3} J=14 \mathrm{~Hz}, 1 \mathrm{H}, \mathrm{CH}\right), 6.34\left(\mathrm{ddd},{ }^{4} \mathrm{~J}=2,{ }^{4} \mathrm{~J}=2,{ }^{3} \mathrm{~J}=14 \mathrm{~Hz}, 1 \mathrm{H}, \mathrm{CH}\right) .-{ }^{13} \mathrm{C}-\mathrm{NMR}$ (62.9 $\mathrm{MHz}_{\mathrm{CDCl}}$, zusätzl. DEPT): $\delta=62.5\left(-, \mathrm{CH}_{2} \mathrm{OH}\right), 77.9\left(\mathrm{C}_{\text {quart }}, \mathrm{C} \equiv \mathrm{C}\right), 81.5(+, \mathrm{C} \equiv \mathrm{C})$, $109.0(+, \mathrm{CH}), 143.7(+, \mathrm{CH})$.

(E)-1-tert-Butyldimethylsiloxypent-2-en-4-in (64): $4.10 \mathrm{~g}$ (50.0 mmol) 23, $6.80 \mathrm{~g}$ (100 mmol) Imidazol und $20.0 \mathrm{ml}$ (50.0 mmol) einer 2.5 M Lösung von tert-Butyldimethyl-

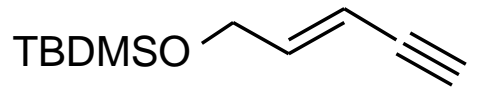
chlorsilan in Hexan wurden in $250 \mathrm{ml}$ wasserfreiem THF gelöst und $5.5 \mathrm{~h}$ bei Raumtemp. gerührt. Es wurde $50 \mathrm{ml}$ Wasser dazugegeben und die organische Phase mit jeweils $50 \mathrm{ml}$ 1 N Salzsäure, ges. Natriumhydrogencarbonatlösung und ges. Natriumchloridlösung gewaschen. Man trocknete über Magnesiumsulfat und entfernte das Lösungsmittel i. Vak. Säulenfiltration über Kieselgel [4.0 × $20 \mathrm{~cm}$, Eluens Pentan/Diethylether $\left.(1: 1), R_{\mathrm{f}}=0.83\right]$ ergaben $9.07 \mathrm{~g}(92 \%) 64$ als farbloses Öl. - IR (Film): $v=3315 \mathrm{~cm}^{-1}, 2956,2858,1653,1472,1377$, 1256, 1132. - $\left.{ }^{1} \mathrm{H}-\mathrm{NMR}\left(250 \mathrm{MHz}, \mathrm{CDCl}_{3}\right): \delta=0.12\left(\mathrm{~s}, 6 \mathrm{H}, \mathrm{SiCH}_{3}\right), 0.92\left[\mathrm{~s}, 9 \mathrm{H}, \mathrm{C} \mathrm{CH}_{3}\right)_{3}\right]$, $2.88\left(\mathrm{~d},{ }^{5} \mathrm{~J}=1 \mathrm{~Hz}, 1 \mathrm{H}, \mathrm{C} \equiv \mathrm{CH}\right), 4.18-4.26\left(\mathrm{~m}, 2 \mathrm{H}, \mathrm{CH}_{2}\right), 5.23\left(\mathrm{dd},{ }^{5} \mathrm{~J}=1,{ }^{3} \mathrm{~J}=11 \mathrm{~Hz}, 1 \mathrm{H}\right.$, $\mathrm{CH}), 6.31\left(\mathrm{dt},{ }^{3} \mathrm{~J}=.11,{ }^{3} \mathrm{~J}=4 \mathrm{~Hz}\right) .-{ }^{13} \mathrm{C}-\mathrm{NMR}\left(62.9 \mathrm{MHz}, \mathrm{CDCl}_{3}\right.$, zusätzl. DEPT): $\delta=-5.4$ $\left(+, \mathrm{SiCH}_{3}\right), 18.3\left[\mathrm{C}_{\text {quart }}, C\left(\mathrm{CH}_{3}\right)_{3}\right], 25.8\left[+, \mathrm{C}\left(\mathrm{CH}_{3}\right)_{3}\right], 62.6\left(-, \mathrm{CH}_{2}\right), 77.4\left(\mathrm{C}_{\text {quart }}, \mathrm{C} \equiv \mathrm{C}\right), 82.0$ $(+, \mathrm{C} \equiv \mathrm{C}), 107.5(+, \mathrm{CH}), 144.3(+, \mathrm{CH})$.

(E)-5-Trimethylsilylpent-2-en-4-in-1-ol (63): Eine Lösung von $4.10 \mathrm{~g}$ (50.0 mmol) 23 in $300 \mathrm{ml}$ wasserfreiem THF wurde auf $0{ }^{\circ} \mathrm{C}$ gekühlt und $46.6 \mathrm{ml}$ (110 mmol) ei-

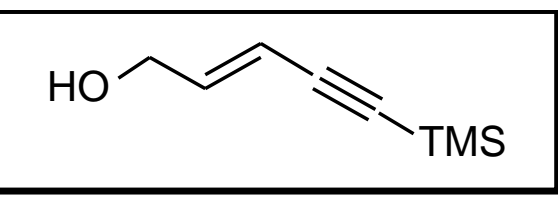
ner 2.36 M Lösung von $n$-Butyllitihum in Hexan zugetropft. Man ließ auf Raumtemp. erwärmen und rührte $12 \mathrm{~h}$. Dann wurde die Reaktionsmischung auf $0{ }^{\circ} \mathrm{C}$ gekühlt und $15.4 \mathrm{ml}$ (110 mmol) Trimethylsilylchlorid zugetropft. Es wurde $5 \mathrm{~h}$ bei Raumtemp. gerührt und die Mischung dann auf $200 \mathrm{ml}$ ges. Ammoniumchloridlösung gegeben. Man extrahierte mit Diethylether $(3 \times 100 \mathrm{ml})$, wusch die vereinigten organischen Extrakte mit ges. Natrium- 
chloridlösung und trochnete über Magnsiumsulfat. Das Lösungsmittel wurde i. Vak. entfernt und man erhielt nach Säulenfiltration über Kieselgel $[4.0 \times 30 \mathrm{~cm}$, Eluens Pentan/Diethylether (1:1), $\left.\mathrm{R}_{\mathrm{f}}=0.38\right] 4.01 \mathrm{~g}(52 \%) 63$ als farbloses Öl. $-{ }^{1} \mathrm{H}-\mathrm{NMR}\left(250 \mathrm{MHz}, \mathrm{CDCl}_{3}\right)$ : $\delta=0.18\left(\mathrm{~s}, 9 \mathrm{H}, \mathrm{SiCH}_{3}\right), 2.07$ (br. s, $\left.1 \mathrm{H}, \mathrm{OH}\right), 4.19$ (dd, $\left.{ }^{4} J=2,{ }^{3} J=5 \mathrm{~Hz}, 1 \mathrm{H}, \mathrm{CH}_{2}\right), 5.75$ $\left(\mathrm{dt},{ }^{4} J=2,{ }^{3} J=16 \mathrm{~Hz}\right), 6.29\left(\mathrm{dt},{ }^{3} J=5,{ }^{3} J=16 \mathrm{~Hz}, 1 \mathrm{H}, \mathrm{CH}\right) .-{ }^{13} \mathrm{C}-\mathrm{NMR}(62.9 \mathrm{MHz}$, $\mathrm{CDCl}_{3}$, zusätzl. DEPT): $\delta=-0.2\left(+, \mathrm{SiCH}_{3}\right), 62.7\left(-, \mathrm{CH}_{2}\right), 95.2\left(\mathrm{C}_{\text {quart }}, \mathrm{C} \equiv \mathrm{C}\right), 103.0\left(\mathrm{C}_{\text {quart }}\right.$, $\mathrm{C} \equiv \mathrm{C}), 110.2(+, \mathrm{CH}), 143.0(+, \mathrm{CH})$.

Versuch zur Darstellung von (E)-1-Acetoxypent-2-en-4-in (62): Bei $0{ }^{\circ} \mathrm{C}$ wurde eine Lösung von $3.28 \mathrm{~g}$ (40.0 mmol) 23, $7.60 \mathrm{ml}(80.0 \mathrm{mmol})$ Acetanhydrid in $8.00 \mathrm{ml}(103 \mathrm{mmol})$ wasserfreiem Pyridin hergestellt. Man ließ auf Raumtemp. kommen und rührte $12 \mathrm{~h}$ weiter. Die Mischung wurde auf $30 \mathrm{ml}$ Diethylether gegeben und $20 \mathrm{ml}$ Wasser hinzugefügt. Man extrahierte mit Diethylether $(2 \times 50 \mathrm{ml})$, trocknete über Magnesiumsulfat und entfernte das Lösungsmittel i. Vak. Das NMR-Spektrum des Rohprodukts zeigte vollständige Zersetzung.

Versuch zur Darstellung von (E)-1-tert-Butoxypent-2-en-4-in (61): In eine Mischung von $4.10 \mathrm{~g}$ (50.0 mmol) 23 und $2.00 \mathrm{~g}$ Amberlyst H-15 in $100 \mathrm{ml}$ Hexan wurde $2.5 \mathrm{~h}$ Isobuten eingeleitet. Es konnte dünnschichtchromatographisch jedoch nicht die Bildung von 61 beobachtet werden. Nach Abfiltrieren des Ionenaustauschers wurde 100 mg Kaliumcarbnoat zugegeben und das Lösungsmittel i. Vak. entfernt. Das Edukt wurde vollständig zurückgewonnen.

trans-1-Hydroxymethyl-2-ethinylcyclopropan (65): Zu einer Lösung von $3.00 \mathrm{ml}(29.3 \mathrm{mmol})$ Diethylzink gab man bei $0{ }^{\circ} \mathrm{C}$ $2.50 \mathrm{ml}(30.0 \mathrm{mmol})$ THF und tropfte dann $4.80 \mathrm{ml}(59.6 \mathrm{mmol})$ Dijodmethan zu. Die Mischung wurde $10 \mathrm{~min}$ bei $0{ }^{\circ} \mathrm{C}$ weitergerührt und dann $0.82 \mathrm{~g}(10.0 \mathrm{mmol}) 23$ zugetropft. Man rührte $1 \mathrm{~h}$

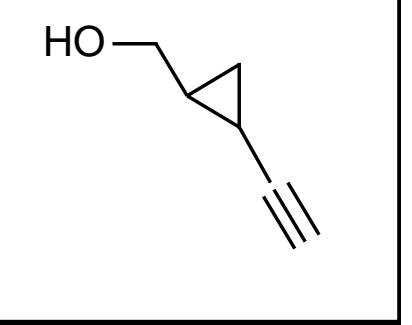
bei $0{ }^{\circ} \mathrm{C}$ und $6 \mathrm{~h}$ bei Raumtemp. Dann gab man $10 \mathrm{ml} 2 \mathrm{~N}$ Salzsäure zu, extrahierte mit Diethylether $(3 \times 15 \mathrm{ml})$, wusch die vereinigten organischen Extrakte mit ges. Natriumthio- 
sulfatlösung, ges. Natriumchloridlösung und trocknete über Magnesiumsulfat. Säulenchromatographie an Kieselgel $\left[2.0 \times 30 \mathrm{~cm}\right.$, Eluens Pentan/Diethylether $\left.(1: 1), R_{\mathrm{f}}=0.33\right]$ ergaben $0.44 \mathrm{~g}(46 \%) 65$ als farbloses Öl. - IR (Film): $v=1000 \mathrm{~cm}^{-1}$, . - ${ }^{1} \mathrm{H}-\mathrm{NMR}$ $\left(250 \mathrm{MHz} \mathrm{CDCl}_{3}\right): \delta=0.65-0.74(\mathrm{~m}, 1 \mathrm{H}, \mathrm{cPr}-\mathrm{H}), 0.82-0.93(\mathrm{~m}, 1 \mathrm{H}, \mathrm{cPr}-\mathrm{H}), 1.08-1.19$ (m, $1 \mathrm{H}, \mathrm{cPr}-\mathrm{H}), 1.34-1.48$ (m, $1 \mathrm{H}, \mathrm{cPr}-\mathrm{H}), 1.80$ (d, $\left.{ }^{4} \mathrm{~J}=2 \mathrm{~Hz}, 1 \mathrm{H}, \mathrm{C} \equiv \mathrm{CH}\right), 3.08$ (br. s, $1 \mathrm{H}$, $\mathrm{OH}), 3.32-3.53\left(\mathrm{~m}, 2 \mathrm{H}, \mathrm{CH}_{2}\right) .-{ }^{13} \mathrm{C}-\mathrm{NMR}\left(62.9 \mathrm{MHz}, \mathrm{CDCl}_{3}\right.$, zusätzl. DEPT): $\delta=4.5$ (-, cPr-C), 12.6 (+, cPr-C), 23.7 (-, cPr-C), $64.4\left(\mathrm{C}_{\text {quart }}, \mathrm{C} \equiv \mathrm{C}\right), 64.7\left(+, \mathrm{CH}_{2}\right), 86.2(-, \mathrm{C} \equiv \mathrm{C}) .-$ MS (70 eV), $m / z(\%): 192(1)\left[\mathrm{M}^{+}\right]$, .

Versuch zur Darstellung von trans-1-Hydroxymethyl-2-ethinylcyclopropan (65): Zu einer Lösung von $427 \mathrm{mg}$ (5.20 mmol) 23 und $0.41 \mathrm{ml}$ (5.09 mmol) Dijodmethan in $4 \mathrm{ml}$ Dichlormethan wurden bei $0{ }^{\circ} \mathrm{C} 5.00 \mathrm{ml}(10.0 \mathrm{mmol})$ einer $2 \mathrm{M}$ Lösung von Trimethylaluminium in Dichlormethan zugetropft. Man ließ die Mischung auf Raumtemp. erwärmen und rührte $12 \mathrm{~h}$ weiter. Das Reaktionsgemisch wurde auf $10 \mathrm{ml}$ einer auf $0{ }^{\circ} \mathrm{C}$ gekühlten $10 \%$ ige Natriumhydroxidlösung gegeben und 30 min gerührt. Man extrahierte mit Diethylether $(3 \times 10 \mathrm{ml})$, trocknete über Magnesiumsulfat und entfernte das Lösungsmittel i. Vak. Es konnten nur $225 \mathrm{mg}(53 \%)$ Edukt zurückgewonnen werden.

Versuch zur Darstellung von trans-1-Hydroxymethyl-2-ethinylcyclopropan (65): Zu einer Lösung von $1.20 \mathrm{ml}(11.7 \mathrm{mmol})$ Diethylzink in $30 \mathrm{ml}$ wasserfreiem Dichlormethan wurden bei $0{ }^{\circ} \mathrm{C} 820 \mathrm{mg}(10.0 \mathrm{mmol}) 23$ zugetropft und $30 \mathrm{~min}$ bei derselben Temperatur gerührt. Dann wurden $1.88 \mathrm{ml}(11.0 \mathrm{mmol}) L(+)$-Weinsäurediethylester zugetropft und $2 \mathrm{~h}$ bei Raumtemp. gerührt. Man kühlte die Mischung auf $-5^{\circ} \mathrm{C}$ ab und tropfte eine Lösung von $5.32 \mathrm{~g}$ (19.9 mmol) Dijodmethan in $10 \mathrm{ml}$ Dichlormethan dazu, ließ auf Raumtemp. erwärem und rührte $4 \mathrm{~d}$ weiter. Zur Mischung wurden $20 \mathrm{ml} 2 \mathrm{~N}$ Salzsäure gegeben, der Niederschlag wurde abfiltriert und mit $200 \mathrm{ml}$ Diethylether gewaschen. Man extrahierte mit Diethylether $(3 \times 50 \mathrm{ml})$, trocknete über Magnsiumsulfat und entfernte das Lösungsmittel i. Vak. Fraktionierende Destillation ergab $646 \mathrm{mg}$ (Sdp. $40-50{ }^{\circ} \mathrm{C} / 1$ Torr) eines Gemisches aus Edukt und Produkt im Verhältnis 1:1. 
trans-1-Hydroxymethyl-2-trimethylsilylethinylcyclopropan

(65a): $\mathrm{Zu}$ einer Lösung von $2.60 \mathrm{ml}$ (25.4 mmol) Diethylzink in $20 \mathrm{ml}$ wasserfreiem Dichlormethan tropfte man bei $0{ }^{\circ} \mathrm{C}$ $2.00 \mathrm{ml}$ (24.8 mmol) Dijodmethan und ließ die Mischung $10 \mathrm{~min}$ weiterrühren. Dann tropfte man $770 \mathrm{mg}(5.00 \mathrm{mmol})$ $63 \mathrm{zu}$, ließ auf Raumtemp. erwärmen und rührte $16 \mathrm{~h}$ weiter.

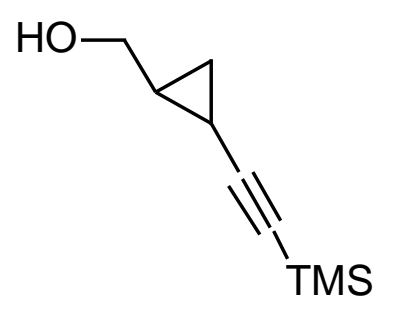

Man gab dann $5 \mathrm{ml}$ ges. Ammoniumchloridlösung dazu und extrahierte mit Dichlormethan $(3 \times 10 \mathrm{ml})$, trocknete über Magnesiumsulfat und entfernte das Lösungsmittel i. Vak. Säulenchromatographie an Kieselgel $\left(2.0 \times 15 \mathrm{~cm}\right.$, Eluens Dichlormethan, $\left.R_{\mathrm{f}}=0.25\right)$ ergab $191 \mathrm{mg}(23 \%)$ 65a als klares Öl. $-{ }^{1} \mathrm{H}-\mathrm{NMR}\left(250 \mathrm{MHz}, \mathrm{CDCl}_{3}\right): \delta=0.04\left(\mathrm{~s}, 9 \mathrm{H}, \mathrm{SiCH}_{3}\right)$, 0.66-0.73 (m, 1 H, cPr-H), 0.83-0.91 (m, 1 H, cPr-H), 1.12-1.20 (m, 1 H, cPr-H), 3.16 (br. s, $\mathrm{OH}), 3.31-3.49\left(\mathrm{~m}, 2 \mathrm{H}, \mathrm{CH}_{2}\right) .-{ }^{13} \mathrm{C}-\mathrm{NMR}\left(62.9 \mathrm{MHz}, \mathrm{CDCl}_{3}\right.$, zusätzl. DEPT): $\delta=0.0$ $\left(-, \mathrm{SiCH}_{3}\right), 5.8$ (+, cPr-C), 13.2 (-, cPr-C), 24.2 (+, cPr-C), $64.8\left(-, \mathrm{CH}_{2}\right), 80.5\left(\mathrm{C}_{\text {quart }}, \mathrm{C} \equiv \mathrm{C}\right)$, $108.7\left(\mathrm{C}_{\text {quart }}, \mathrm{C} \equiv \mathrm{C}\right)$.

trans-1-tert-Butyldimethylsiloxymethyl-2-ethinylcyclopropan (65b): Eine Mischung aus $342 \mathrm{mg}$ (3.56 mmol) 65, $374 \mathrm{mg}$ (5.49 mmol) Imidazol und $2.00 \mathrm{ml}(5.00 \mathrm{mmol})$ einer 2.5 M Lösung von tert-Butyldimethylsilylchlorid in Hexan wurden in $5 \mathrm{ml}$ wasserfreiem DMF $16 \mathrm{~h}$ bei Raumtemp.

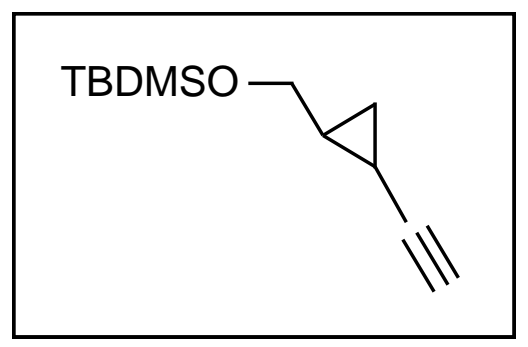
gerührt. Man gab $20 \mathrm{ml}$ Diethylether und $20 \mathrm{ml}$ Wasser dazu, wusch mit Wasser $(2 \times 10 \mathrm{ml})$, $10 \mathrm{ml}$ ges. Natriumchloridlösung und entfernte das Lösungsmittel i. Vak. Säulenfiltration über Kieselgel $\left(2.0 \times 15 \mathrm{~cm}\right.$, Eluens Pentan, $\left.R_{\mathrm{f}}=0.27\right)$ ergab $128 \mathrm{mg}(17 \%) 65 \mathrm{~b}$ als klares Öl. - IR (Film): v = $3316 \mathrm{~cm}^{-1}, 2955,2930,2885,2857,1471,1256,1092,837 .-{ }^{1} \mathrm{H}-\mathrm{NMR}$ $\left(250 \mathrm{MHz}, \mathrm{CDCl}_{3}\right): \delta=0.03\left(\mathrm{~s}, 6 \mathrm{H}, \mathrm{SiCH}_{3}\right), 0.70-0.81(\mathrm{~m}, 2 \mathrm{H}, \mathrm{cPr}-\mathrm{H}), 0.85[\mathrm{~s}, 9 \mathrm{H}$, $\left.\mathrm{C}\left(\mathrm{CH}_{3}\right)_{3}\right] 1.13-1.24(\mathrm{~m}, 1 \mathrm{H}, \mathrm{cPr}-\mathrm{H}), 1.28-1.41(\mathrm{~m}, 1 \mathrm{H}, \mathrm{cPr}-\mathrm{H}), 1.77\left(\mathrm{~d},{ }^{4} \mathrm{~J}=1 \mathrm{~Hz}, 1 \mathrm{H}\right.$, $\mathrm{CH}), 3.57\left(\mathrm{~d},{ }^{3} \mathrm{~J}=5 \mathrm{~Hz}, 2 \mathrm{H}, \mathrm{CH}_{2}\right) .-{ }^{13} \mathrm{C}-\mathrm{NMR}\left(62.9 \mathrm{MHz}, \mathrm{CDCl}_{3}\right.$, zusätzl. DEPT): $\delta=-$ $5.3\left(-, \mathrm{SiCH}_{3}\right), 3.6(-, \mathrm{cPr}-\mathrm{C}), 12.1$ (+. cPr-C), $18.3\left[\mathrm{C}_{\text {quart }}, \mathrm{C}\left(\mathrm{CH}_{3}\right)_{3}\right], 25.8\left[-, \mathrm{C}\left(\mathrm{CH}_{3}\right)_{3}\right], 63.5$ $\left(+, \mathrm{CH}_{2}\right), 63.9\left(\mathrm{C}_{\text {quart }}, \mathrm{C} \equiv \mathrm{C}\right), 86.8(-, \mathrm{C} \equiv \mathrm{C}) .-\mathrm{MS}(70 \mathrm{eV}), \mathrm{m} / z(\%): 210$ (1) $\left[\mathrm{M}^{+}\right], 153$ (17) $\left[\mathrm{M}^{+}-\mathrm{C}\left(\mathrm{CH}_{3}\right)_{3}\right], 101$ (24) $\left[\mathrm{OSiC}\left(\mathrm{CH}_{3}\right)_{3}{ }^{+}\right], 75$ (100) $\left[\mathrm{HOSi}\left(\mathrm{CH}_{3}\right)_{2}{ }^{+}\right] .-\mathrm{C}_{12} \mathrm{H}_{22} \mathrm{OSi}$ : ber. 210.1439, gef. 210.1439 (MS). 


\subsection{Kupplungsreaktionen}

\subsubsection{Allgemeine Arbeitsvorschriften für Kupplungsreaktionen}

Allgemeine Arbeitsvorschrift zur Darstellung von Chloreninen aus Alkinen und trans-1,2Dichlorethen (AAV1): Eine Mischung aus Tetrakis-(triphenylphosphin)-palladium(0), trans1,2-Dichlorethen, Piperidin und Kupfer(I)iodid in Benzol oder lösungsmittelfrei wurden bei $0{ }^{\circ} \mathrm{C}$ durch 10 minütiges Hindurchleiten von Stickstoff von gelöstem Sauerstoff befreit. Man tropfte dann eine Lösung des Alkins in Benzol oder das reine Alkin hinzu, ließ auf Raumtemp. erwärmen und rührte so lange, bis dünnschichtchromatographisch kein Alkin mehr nachgewiesen werden konnte. Die Mischung wurde über eine Säule filtriert, die von unten nach oben mit einer jeweils $5 \mathrm{~cm}$ starken Schicht aus Kieselgel und Celite gefüllt war. Das Rohprodukt wurde säulenchromatographisch mit geeigneten Lösungsmittelgemischen oder durch fraktionierende Destillation gereinigt.

Allgemeine Arbeitsvorschrift zur Darstellung von Endiinen aus Chloreninen und Alkinen (AAV2): Zu einer Mischung von Bis-(triphenylphosphin)-palladium(II)-chlorid, Piperidin, dem Chlorenin und dem Alkin gab man bei $0{ }^{\circ} \mathrm{C}$ Kupfer(I)-iodid. Man ließ auf Raumtemp. erwärmen und rührte so lange, bis säulenchromatographisch keine Edukte mehr nachweisbar waren. Man filtrierte die Reaktionsmischung über $5 \mathrm{~cm}$ Celite, entfernte das Piperidin i. Vak. und reinigte das Rohprodukt mit geeigneten Lösungsmittelgemischen an Kieselgel.

\subsubsection{Enine}

(E)-5-Chlor-1-(tert-butyl-dimethylsiloxy)-pent-2-in-4-

en (93): Nach AAV1 wurden $541 \mathrm{mg}(2.8 \mathrm{~mol} \%)$ Tetrakis-(triphenylphosphin)-palladium(0), $6.94 \mathrm{ml}$

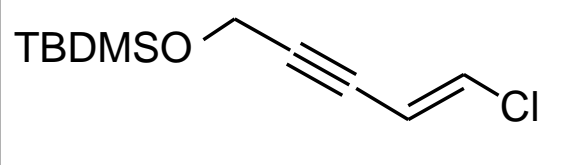
(90.0 mmol) trans-1,2-Dichlorethen, $3.50 \mathrm{ml}$ (35.4 mmol) Piperidin und $178 \mathrm{mg}$ (5.6 mol\%) Kupfer(I)-iodid in $4 \mathrm{ml}$ Benzol mit einer Lösung von $2.84 \mathrm{~g}$ (16.7 mmol) tert-Butyldimethylsiloxy-2-propin in $6 \mathrm{ml}$ Benzol $2.5 \mathrm{~h}$ bei Raumtemp. umgesetzt. Säulenfiltration über Kieselgel [3 $\times 20 \mathrm{~cm}$, Eluens Pentan/Diethylether $\left.(10: 1), R_{\mathrm{f}}=0.71\right]$ ergab $3.29 \mathrm{~g}(85 \%) 93$ 
als farbloses Öl. - IR (Film): v =3385 cm $\mathrm{cm}^{-1}$, 2955, 2857, 2189, 1717, 1471, 1362, 1265, 1166, 1072, 1005, 919. - ${ }^{1} \mathrm{H}-\mathrm{NMR}\left(250 \mathrm{MHz}^{\mathrm{C}} \mathrm{CDCl}_{3}\right): \delta=0.11\left(\mathrm{~s}, 6 \mathrm{H}, \mathrm{SiCH}_{3}\right), 0.90[\mathrm{~s}$, $\left.9 \mathrm{H}, \mathrm{C}\left(\mathrm{CH}_{3}\right)_{3}\right], 4.41\left(\mathrm{~s}, 2 \mathrm{H}, \mathrm{CH}_{2}\right), 5.94\left(\mathrm{dt},{ }^{3} \mathrm{~J}=13,{ }^{5} \mathrm{~J}=2 \mathrm{~Hz}, 1 \mathrm{H}, \mathrm{CH}\right), 6.50\left(\mathrm{~d},{ }^{3} \mathrm{~J}=13 \mathrm{~Hz}\right.$, $1 \mathrm{H}, \mathrm{CH}) .-{ }^{13} \mathrm{C}-\mathrm{NMR}\left(62.9 \mathrm{MHz}, \mathrm{CDCl}_{3}\right.$, zusätzl. DEPT): $\delta=-5.2\left(-, \mathrm{SiCH}_{3}\right), 18.3\left[\mathrm{C}_{\text {quart }}\right.$, $\left.\mathrm{C}\left(\mathrm{CH}_{3}\right)_{3}\right], 25.8\left[-, \mathrm{C}\left(\mathrm{CH}_{3}\right)_{3}\right], 52.0\left(+, \mathrm{CH}_{2}\right), 79.8\left(\mathrm{C}_{\text {quart }}, \mathrm{C} \equiv \mathrm{C}\right), 90.5\left(\mathrm{C}_{\text {quart }}, \mathrm{C} \equiv \mathrm{C}\right), 113.4(-$,

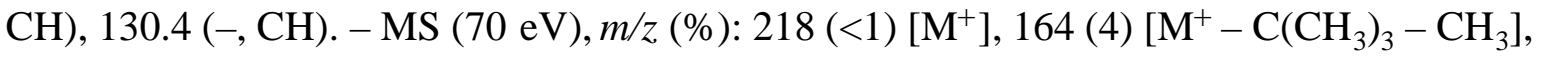
$149(8)\left[\mathrm{M}^{+}-\mathrm{C}\left(\mathrm{CH}_{3}\right)_{3}-2 \mathrm{CH}_{3}\right], 130$ (95), 113 (90), 102 (88), 81 (87), 51 (100).

(E)-1-tert-Butyloxy-5-chlor-pent-2-in-4-en (92): Nach AAV1 wurden $578 \mathrm{mg}$ (4.7mol\%) Tetrakis-(triphenylphosphin)-palladium(0), $3.86 \mathrm{ml}(50.0 \mathrm{mmol})$ trans-1,2Dichlorethen, $1.97 \mathrm{ml} \quad(19.9 \mathrm{mmol})$ Piperidin und

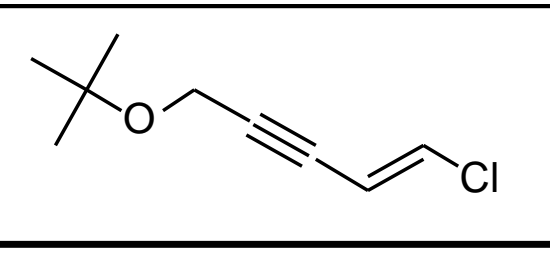
$190 \mathrm{mg}$ (9.3 mol\%) Kupfer(I)-iodid in 4 ml Benzol mit einer Lösung von 1,21 g (10.7 mmol) tert-Butyloxy-2-propin in $5 \mathrm{ml}$ Benzol $2 \mathrm{~h}$ bei Raumtemp. umgesetzt. Säulenfiltration über Kieselgel [3.0 × $15 \mathrm{~cm}$, Eluens Pentan/Diethylether (10:1), $\left.R_{\mathrm{f}}=0.64\right]$ ergab $1.51 \mathrm{~g}(82 \%)$ 92 als farbloses Öl. - IR (Film): $v=2977 \mathrm{~cm}^{-1}, 2227,2189,1714,1602,1472,1370,1167$, 920. - ${ }^{1} \mathrm{H}-\mathrm{NMR}\left(250 \mathrm{MHz}, \mathrm{CDCl}_{3}\right): \delta=1.23\left[\mathrm{~s}, 9 \mathrm{H}, \mathrm{C}\left(\mathrm{CH}_{3}\right)_{3}\right], 4.15\left(\mathrm{~d},{ }^{5} \mathrm{~J}=2 \mathrm{~Hz}, 2 \mathrm{H}\right.$, $\left.\mathrm{CH}_{2}\right), 5.92\left(\mathrm{dt},{ }^{3} \mathrm{~J}=14,{ }^{5} \mathrm{~J}=2 \mathrm{~Hz}, 1 \mathrm{H}, \mathrm{CH}\right), 6.49\left(\mathrm{~d},{ }^{3} \mathrm{~J}=14 \mathrm{~Hz}, 1 \mathrm{H}, \mathrm{CH}\right) .-{ }^{13} \mathrm{C}-\mathrm{NMR}$ (62.9 MHz, $\mathrm{CDCl}_{3}$, zusätzl. DEPT): $\delta=27.3\left[+, \mathrm{C}\left(\mathrm{CH}_{3}\right)_{3}\right], 50.8\left(-, \mathrm{CH}_{2}\right), 74.3\left[\mathrm{C}_{\text {quart }}\right.$, $\left.C\left(\mathrm{CH}_{3}\right)_{3}\right], 79.7\left(\mathrm{C}_{\text {quart }}, \mathrm{C} \equiv \mathrm{C}\right), 89.6\left(\mathrm{C}_{\text {quart }}, \mathrm{C} \equiv \mathrm{C}\right), 113.5(+, \mathrm{CH}), 130.4(+. \mathrm{CH}) .-\mathrm{MS}(70 \mathrm{eV})$, $m / z(\%): 172$ (2) $\left[\mathrm{M}^{+}\right], 130$ (89), 115 (86) $\left[\mathrm{M}^{+}-\mathrm{C}\left(\mathrm{CH}_{3}\right)_{3}\right], 113$ (100), 102 (72), 59 (66), 57 (72).

(E)-5-Chlor-pent-2-in-4-en-1-ol (94): Nach AAV1 wurden $540 \mathrm{mg} \quad(4.7 \mathrm{~mol} \%) \quad$ Tetrakis-(triphenylphosphin)-palladium(0), $3.47 \mathrm{ml}$ (45.0 mmol) trans-1,2-Dichlorethen, $1.77 \mathrm{ml}$

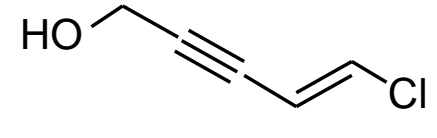
(17.9 mmol) Piperidin und $171 \mathrm{mg}(9.3 \mathrm{~mol} \%)$ Kupfer(I)-iodid in $5 \mathrm{ml} \mathrm{Benzol} \mathrm{mit} \mathrm{einer} \mathrm{Lö-}$ sung von $504 \mathrm{mg}(8.99 \mathrm{mmol})$ Propargylalkohol in $5 \mathrm{ml}$ Benzol $2 \mathrm{~h}$ bei Raumtemp. umgesetzt. Säulenfiltration über Kieselgel $\left(3.0 \times 15 \mathrm{~cm}\right.$, Eluens Dichlormethan, $\left.R_{\mathrm{f}}=0.42\right)$ ergab $522 \mathrm{mg}(50 \%) 94$ als farbloses Öl. $-{ }^{1} \mathrm{H}-\mathrm{NMR}\left(250 \mathrm{MHz}, \mathrm{CDCl}_{3}\right): \delta=2.77$ (br. s, $\left.1 \mathrm{H}, \mathrm{OH}\right)$, 
$4.34\left(\mathrm{~d},{ }^{5} \mathrm{~J}=2 \mathrm{~Hz}, 2 \mathrm{H}, \mathrm{CH}_{2}\right), 5.95\left(\mathrm{dt},{ }^{3} \mathrm{~J}=14,{ }^{5} \mathrm{~J}=2 \mathrm{~Hz}, \mathrm{CH}\right), 6.53\left(\mathrm{~d},{ }^{3} \mathrm{~J}=14 \mathrm{~Hz}, 1 \mathrm{H}\right.$, $\mathrm{CH}) .-{ }^{13} \mathrm{C}-\mathrm{NMR}\left(62.9 \mathrm{MHz}, \mathrm{CDCl}_{3}\right.$, zusätzl. DEPT): $\delta=51.1\left(+, \mathrm{CH}_{2}\right), 80.5\left(\mathrm{C}_{\text {quart }}, \mathrm{C} \equiv \mathrm{C}\right)$, $89.8\left(\mathrm{C}_{\text {quart }}, \mathrm{C} \equiv \mathrm{C}\right), 113.1(-, \mathrm{CH}), 131.0(-, \mathrm{CH})$.

(E)-4-Chlor-1-Cyclopropylbut-3-en-1-in (95): Nach AAV1 wurden $2.31 \mathrm{~g}(2.0 \mathrm{~mol} \%)$ Tetrakis-(triphenylphosphin)-palladium(0), $30.8 \mathrm{ml} \mathrm{(399} \mathrm{mmol)} \mathrm{trans-1,2-Dichlorethen,} 20.0 \mathrm{ml}$

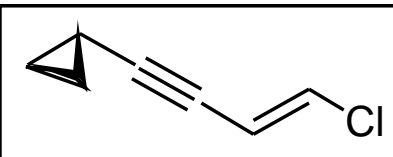
(202 mmol) Piperidin und 970 mg (5.0 mol\%) Kupfer(I)-iodid mit 6.70 g (101 mmol) Cyclopropylacetylen in einer Pyrexflsche $2 \mathrm{~h}$ bei Raumtemp. umgesetzt. Fraktionierende Destillation (Sdp. $32-35^{\circ} \mathrm{C} / 1$ Torr) ergab $10.5 \mathrm{~g}$ (82\%) 95 als farbloses Öl. . - IR (Film): $v=3074 \mathrm{~cm}^{-1}, 2209,1700,1585,1357,1232,1029,917 .-{ }^{1} \mathrm{H}-\mathrm{NMR}\left(250 \mathrm{MHz}, \mathrm{CDCl}_{3}\right)$ : $\delta=0.83-0.96(\mathrm{~m}, 4 \mathrm{H}, \mathrm{cPr}-\mathrm{H}), 1.23-1.41(\mathrm{~m}, 1 \mathrm{H}, \mathrm{cPr}-\mathrm{H}), 5.86\left(\mathrm{dd},{ }^{3} J=14,{ }^{5} \mathrm{~J}=2 \mathrm{~Hz}, 1 \mathrm{H}\right.$, $\mathrm{CH}), 6.40\left(\mathrm{~d},{ }^{3} \mathrm{~J}=.14 \mathrm{~Hz}, 1 \mathrm{H}, \mathrm{CHCl}\right) .-{ }^{13} \mathrm{C}-\mathrm{NMR}\left(62.9 \mathrm{MHz}, \mathrm{CDCl}_{3}\right.$, zusätzl. DEPT): $\delta=0.10\left(-\right.$, cPr-C), 8.56 (+, cPr-C), $70.900\left(\mathrm{C}_{\text {quart }}, \mathrm{C}-1\right), 96.39\left(\mathrm{C}_{\text {quart }}, \mathrm{C}-2\right), 114.15(-, \mathrm{CH})$, 128.82 (-, $\mathrm{CHCl}) .-\mathrm{MS}(70 \mathrm{eV}), \mathrm{m} / z(\%): 126(78)\left[\mathrm{M}^{+}\right], 91(100)\left[\mathrm{M}^{+}-\mathrm{Cl}\right], 65$ (42). $\mathrm{C}_{7} \mathrm{H}_{7} \mathrm{Cl}$ (126.59): ber. C 66.42, H 5.57, gef. C 66.28, H 5.72.

(E)-4-Chlor-1-Benzyloxymethylcyclopropylbut-3-en1-in (96): Nach AAV1 wurden $231 \mathrm{mg}$ (4.7mol\%) Tetrakis-(triphenylphosphin)-palladium(0),

$0.77 \mathrm{ml}$

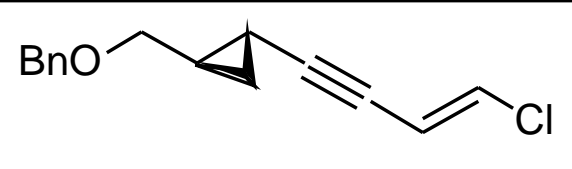

(9.98 mmol) trans-1,2-Dichlorethen, $\quad 0.40 \mathrm{ml} \quad(4.04 \mathrm{mmol}) \quad$ Piperidin und $40.0 \mathrm{mg}$ (9.3 mol\%) Kupfer(I)-iodid in $2 \mathrm{ml}$ Benzol mit einer Lösung von $372 \mathrm{mg}$ (2.00 mmol) XX in $2 \mathrm{ml} \mathrm{Benzol} 2.5 \mathrm{~h}$ bei Raumtemp. umgesetzt. Säulenfiltration über Kieselgel $[1.5 \times 10 \mathrm{~cm}$, Eluens Pentan/Diethylether (10: 1), $R_{\mathrm{f}}=0.65$ ] ergab $235 \mathrm{mg}(48 \%) 96$ als farbloses Ö1. - IR (Film): $v=3446 \mathrm{~cm}^{-1}$, 3030, 2860, 2248, 2154, 1700, 1652, 1558, 1495, 1454, 1361, 1204, 1074, 909, 732, 698, 649. - ${ }^{1} \mathrm{H}-\mathrm{NMR}\left(250 \mathrm{MHz}, \mathrm{CDCl}_{3}\right): \delta=0.79-0.87$ (m, $\left.1 \mathrm{H}, \mathrm{cPr}-\mathrm{H}\right)$, 0.94-1.01 (m, $1 \mathrm{H}, \mathrm{cPr}-\mathrm{H}), 1.29-1.33$ (m, $1 \mathrm{H}, \mathrm{cPr}-\mathrm{H}), 1.47-1.54$ (m, $1 \mathrm{H}, \mathrm{cPr}-\mathrm{H}), 3.36$ (dd, $\left.{ }^{2} J=10,{ }^{3} J=7 \mathrm{~Hz}, 1 \mathrm{H}, \mathrm{CH}_{2} \mathrm{OBn}\right), 3.41\left(\mathrm{dd},{ }^{2} J=10,{ }^{3} \mathrm{~J}=7 \mathrm{~Hz}, 1 \mathrm{H}, \mathrm{CH}_{2} \mathrm{OBn}\right), 4.54$ (s, $2 \mathrm{H}$, $\left.\mathrm{OCH}_{2} \mathrm{Ph}\right), 5.88\left(\mathrm{dd},{ }^{5} J=2,{ }^{3} J=14 \mathrm{~Hz}, 1 \mathrm{H}, \mathrm{CH}\right), 6.43\left(\mathrm{~d},{ }^{3} J=14 \mathrm{~Hz}, 1 \mathrm{H}, \mathrm{CHCl}\right), 7.35(\mathrm{~m}$, $5 \mathrm{H}, \mathrm{Ph}-\mathrm{H}) .-{ }^{13} \mathrm{C}-\mathrm{NMR}\left(62.9 \mathrm{MHz}, \mathrm{CDCl}_{3}\right.$, zusätzl. DEPT): $\delta=5.8(-, \mathrm{cPr}-\mathrm{C}), 13.3(+$, 
cPr-C), 21.9 (-, cPr-C), $71.7\left(\mathrm{C}_{\text {quart }}, \mathrm{C}-1\right), 71.9$ (+, $\left.\mathrm{CH}_{2}\right), 72.5\left(+, \mathrm{CH}_{2}\right), 94.9\left(\mathrm{C}_{\text {quart }}, \mathrm{C}-2\right)$,

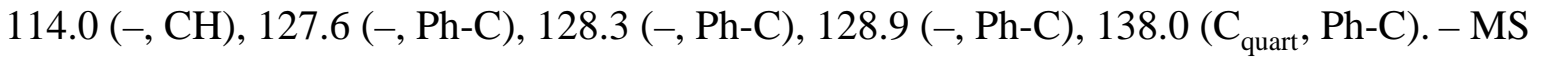
(DCI, $70 \mathrm{eV}), m / z(\%): 758$ (2) $\left[2 \mathrm{M}^{+}+\mathrm{NH}_{4}^{+}\right], 388(100)\left[\mathrm{M}^{+}+\mathrm{NH}_{4}^{+}\right] .-\mathrm{C}_{15} \mathrm{H}_{15} \mathrm{ClO}$ (246.74).

(E,E)-1-tert-Butyldimethylsiloxy-7-Chlor-hept2,6-dien-4-in (97): Nach AAV1 wurden $62.0 \mathrm{mg}(1.8 \mathrm{~mol} \%)$ Tetrakis-(triphenylphos-

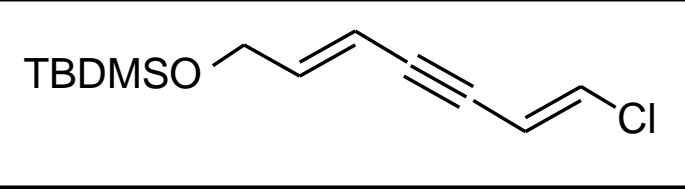
phin)-palladium(0), $0.77 \mathrm{ml}(9.98 \mathrm{mmol})$ trans-1,2-Dichlorethen, $0.39 \mathrm{ml}(3.94 \mathrm{mmol})$ Piperidin und $27.0 \mathrm{mg}(6.9 \mathrm{~mol} \%)$ Kupfer(I)-iodid in $2 \mathrm{ml} \mathrm{Benzol} \mathrm{mit} 0.40 \mathrm{~g}$ (2.04 mmol) $641.5 \mathrm{~h}$ bei Raumtemp. umgesetzt. Säulenfiltration über Kieselgel [2.0 $\times 15 \mathrm{~cm}$, Eluens Pentan/Diethylether (20:1), $\left.R_{\mathrm{f}}=0.79\right]$ ergab $0.47 \mathrm{~g}(89 \%) 97$ als farbloses Ö1. $-{ }^{1} \mathrm{H}-\mathrm{NMR}$ $\left(250 \mathrm{MHz}, \mathrm{CDCl}_{3}\right): \delta=0.07\left(\mathrm{~s}, 6 \mathrm{H}, \mathrm{SiCH}_{3}\right), 0.91\left[\mathrm{~s}, 9 \mathrm{H}, \mathrm{C}\left(\mathrm{CH}_{3}\right)_{3}\right], 4.20-4.28(\mathrm{~m}, 4 \mathrm{H}$, $\left.\mathrm{CH}_{2}\right), 5.83\left(\mathrm{dd},{ }^{3} \mathrm{~J}=14,{ }^{5} \mathrm{~J}=2 \mathrm{~Hz}, 1 \mathrm{H}, \mathrm{CH}\right), 6.04\left(\mathrm{dd},{ }^{3} \mathrm{~J}=14,{ }^{5} \mathrm{~J}=2 \mathrm{~Hz}, 1 \mathrm{H}, \mathrm{CH}\right), 6.23(\mathrm{dt}$, $\left.{ }^{3} J=14,{ }^{3} J=3 \mathrm{~Hz}, 1 \mathrm{H}, \mathrm{CH}\right), 6.50\left(\mathrm{~d},{ }^{3} J=14 \mathrm{~Hz}, 1 \mathrm{H}, \mathrm{CH}\right) .-{ }^{13} \mathrm{C}-\mathrm{NMR}(62.9 \mathrm{MHz}$,

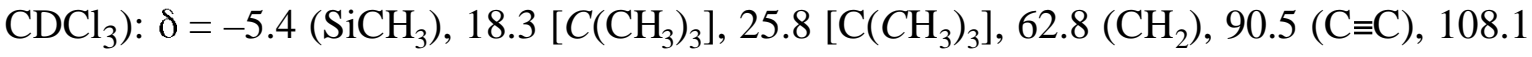
$(\mathrm{C} \equiv \mathrm{C}), 114.0(\mathrm{C}=\mathrm{C}), 128.3(\mathrm{C}=\mathrm{C}), 129.6(\mathrm{C}=\mathrm{C}), 143.2(\mathrm{C}=\mathrm{C})$.

\subsubsection{Endiine}

1,6-Bis(tert-butyl)hex-3-en-1,5-diin (88): Zu einer Lösung von $930 \mathrm{mg}$ (11.3 mmol) 3,3-Dimethyl-1-butin in $10 \mathrm{ml}$ wasserfreiem THF wurde bei $-70^{\circ} \mathrm{C} 5.00 \mathrm{ml}$ (11.8 mmol) einer 2.36 M Lösung von $n$-Butyllithium in Hexan zugetopft und die Mischung innerhalb von $3 \mathrm{~h}$

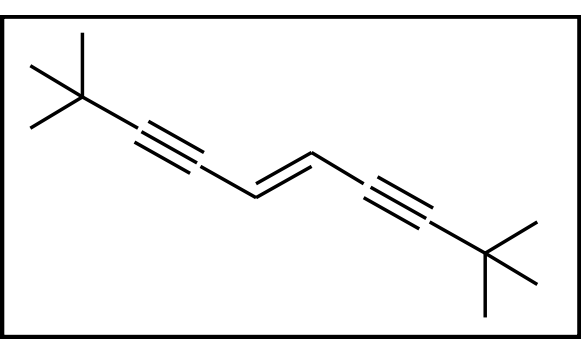
auf $0^{\circ} \mathrm{C}$ erwärmt. Dann tropfte man eine Lösung von $1.64 \mathrm{~g}(12.0 \mathrm{mmol})$ Zinkdichlorid in $10 \mathrm{ml} \mathrm{THF} \mathrm{zu} \mathrm{und} \mathrm{rührte} 30 \mathrm{~min}$ bei $0^{\circ} \mathrm{C}$ weiter. Danach gab man $470 \mathrm{mg}(8.0 \mathrm{~mol} \%)$ Tetrakis-(triphenylphosphin)-palladium(0) und $940 \mathrm{mg}$ (5.08 mmol) 1,2-Dibromethen hinzu, ließ auf Raumtemp. erwärmen und rührte $12 \mathrm{~h}$ bei dieser Temperatur. Zur Mischung wurde $20 \mathrm{ml}$ Pentan und $20 \mathrm{ml}$ ges. Ammoniumchloridlösung gegeben. Man extrahierte mit Pentan 
$(3 \times 10 \mathrm{ml})$, trocknete über Magnesiumsulfat und entfernte das Lösungsmittel i. Vak. Säulenchromatographie an Kieselgel $\left(3 \times 20 \mathrm{~cm}\right.$, Eluens Pentan, $\left.R_{\mathrm{f}}=0.9\right)$ ergab $582 \mathrm{mg}(61 \%)$ 88 als weiße Kristalle, Schmp. $67^{\circ} \mathrm{C} .-{ }^{1} \mathrm{H}-\mathrm{NMR}\left(250 \mathrm{MHz}, \mathrm{CDCl}_{3}\right): \delta=1.24[\mathrm{~s}, 18 \mathrm{H}$, $\left.\mathrm{C}\left(\mathrm{CH}_{3}\right)_{3}\right], 5.87(\mathrm{~s}, 2 \mathrm{H}, \mathrm{CH}) .-{ }^{13} \mathrm{C}-\mathrm{NMR}\left(62.9 \mathrm{MHz}, \mathrm{CDCl}_{3}\right): \delta=31.0\left[\mathrm{C}\left(\mathrm{CH}_{3}\right)_{3}\right], 68.0$ $\left[C\left(\mathrm{CH}_{3}\right)_{3}\right], 76.2(\mathrm{C} \equiv \mathrm{C}), 102.5(\mathrm{C} \equiv \mathrm{C}), 119.9(\mathrm{CH})$. Die übrigen Daten stimmten mit den in der Literatur gefundenen überein.

1,6-Bis(phenyl)hex-3-en-1,5-diin (89): Zu einer Lösung von $1.08 \mathrm{~g}$ (10.6 mmol) Phenylacetylen in $20 \mathrm{ml}$ wasserfreiem $\mathrm{THF}$ wurde bei $-70^{\circ} \mathrm{C}$ $5.08 \mathrm{ml}(12.0 \mathrm{mmol})$ einer $2.36 \mathrm{M}$ Lösung von n-Butyllithium in Hexan zugetropft und die Mischung innerhalb von $3 \mathrm{~h}$ auf $0^{\circ} \mathrm{C}$ erwärmt. Dann

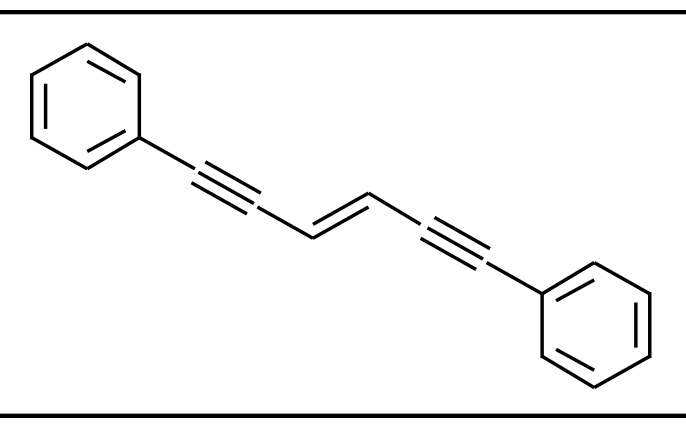
tropfte man eine Lösung von $1.70 \mathrm{~g}$ (12.5 mmol) Zinkdichlorid in $10 \mathrm{ml}$ THF zu und rührte $30 \mathrm{~min}$ bei $0^{\circ} \mathrm{C}$ weiter. Danach gab man $593 \mathrm{mg}(10 \mathrm{~mol} \%)$ Tetrakis-(triphenylphosphin)palladium(0) und $933 \mathrm{mg}$ (5.02 mmol) 1,2-Dibromethen hinzu, ließ auf Raumtemp. erwärmen und rührte $12 \mathrm{~h}$ bei dieser Temperatur. Zur Mischung wurde $20 \mathrm{ml}$ Pentan und $20 \mathrm{ml}$ ges. Ammoniumchloridlösung gegeben. Man extrahierte mit Pentan $(3 \times 10 \mathrm{ml})$, trocknete über Magnesiumsulfat und entfarnte das Lösungsmittel i. Vak. Säulenchromatographie an Kieselgel $\left(3 \times 20 \mathrm{~cm}\right.$, Eluens Pentan, $\left.R_{\mathrm{f}}=0.48\right)$ ergab $690 \mathrm{mg}(60 \%) 89$ als weißen Feststoff, Schmp $63{ }^{\circ} \mathrm{C} .-{ }^{1} \mathrm{H}-\mathrm{NMR}\left(250 \mathrm{MHz}, \mathrm{CDCl}_{3}\right): \delta=6.34(\mathrm{~s}, 2 \mathrm{H}, \mathrm{CH}), 7.31-7.42$ (m, $10 \mathrm{H}, \mathrm{Ph}-\mathrm{H}) .-{ }^{13} \mathrm{C}-\mathrm{NMR}\left(62.9 \mathrm{MHz}, \mathrm{CDCl}_{3}\right.$, zusätzl. DEPT): $\delta=88.1\left(\mathrm{C}_{\text {quart }}, \mathrm{C} \equiv \mathrm{C}\right), 94.8$ $\left(\mathrm{C}_{\text {quart }}, \mathrm{C} \equiv \mathrm{C}\right), 120.7(+, \mathrm{CH}), 122.9\left(\mathrm{C}_{\text {quart }}, \mathrm{Ph}-\mathrm{C}\right), 128.4(+, \mathrm{Ph}-\mathrm{C}), 128.6(+, \mathrm{Ph}-\mathrm{C}), 131.6(+$, $\mathrm{Ph}-\mathrm{C})$.

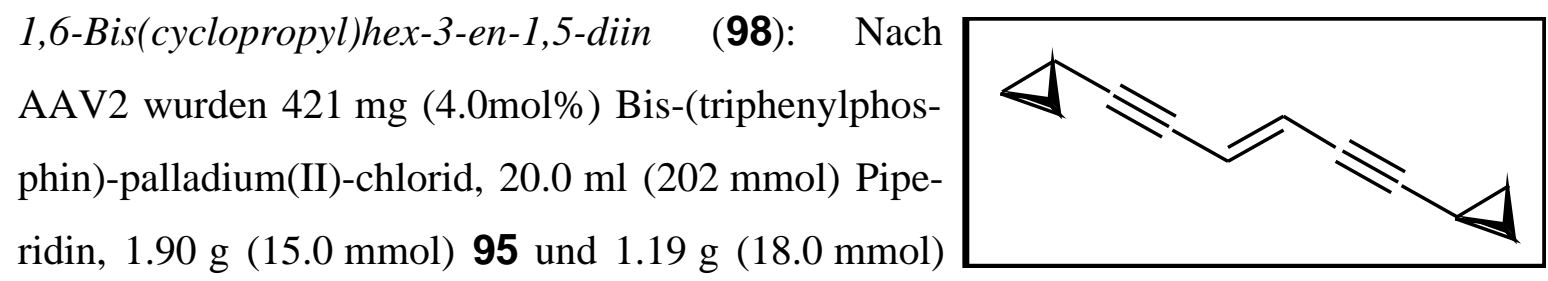
Cyclopropylacetylen bei $0{ }^{\circ} \mathrm{C}$ mit $266 \mathrm{mg}$ (9.3mol\%) Kupfer(I)-iodid $2 \mathrm{~h}$ zur Reaktion ge- 
bracht. Die säulenchromatographische Reinigung an Kieselgel $(3.0 \times 15$ cm, Eluens Pentan, $\left.R_{\mathrm{f}}=0.26\right)$ ergab $2.23 \mathrm{~g}(95 \%) 98$ als farblose Kristalle, Schmp. $72{ }^{\circ} \mathrm{C} .-{ }^{1} \mathrm{H}-\mathrm{NMR}$ $\left(250 \mathrm{MHz} \mathrm{CDCl}_{3}\right): \delta=0.85-0.97(\mathrm{~m}, 8 \mathrm{H}, \mathrm{cPr}-\mathrm{H}), 1.24-1.43(\mathrm{~m}, 2 \mathrm{H}, \mathrm{cPr}-\mathrm{H}), 5.82(\mathrm{~s}, 2 \mathrm{H}$, $\mathrm{CH}) .-{ }^{13} \mathrm{C}-\mathrm{NMR}\left(62.9 \mathrm{MHz}, \mathrm{CDCl}_{3}\right.$, zusätzl. DEPT): $\delta=0.3(+, \mathrm{cPr}-\mathrm{C}), 8.7$ (-, cPr-C), 74.5 (C quart C-1, C-6), 98.1 (C $\left.\mathrm{C}_{\text {quart }}, \mathrm{C}-2, \mathrm{C}-5\right), 120.1$ (+, C-3, C-4). - MS (70 eV), m/z (\%): $156(100)\left[\mathrm{M}^{+}\right], 128(55), 115(100)\left[\mathrm{M}^{+}-\mathrm{C}_{3} \mathrm{H}_{4}\right]$.

1-(Benzyloxymethylcyclopropyl)-6-cyclopropylhex-3-en-1,5-diin (99): Nach AAV2 wurden $\quad 421 \mathrm{mg} \quad(4.0 \mathrm{~mol} \%) \quad$ Bis(triphenylphosphin)-palladium(II)-chlorid,

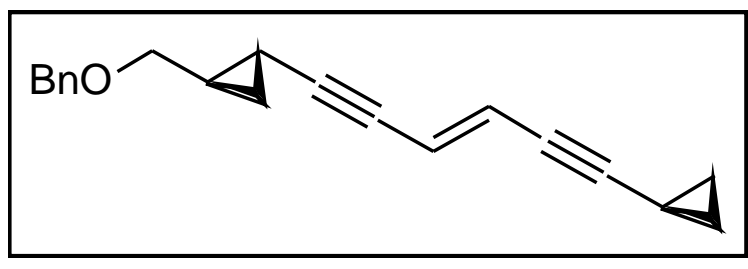
$20.0 \mathrm{ml}$ (202 mmol) Piperidin, $1.90 \mathrm{~g}$ (15.0 mmol) 96 und $2.79 \mathrm{~g}(15.0 \mathrm{mmol})$ bei $0{ }^{\circ} \mathrm{C}$ mit 265 mg (9.3mol\%) Kupfer(I)-iodid 2.5 h zur Reaktion gebracht. Die säulenchromatographische Reinigung an Kieselgel $\left[3.0 \times 15 \mathrm{~cm}\right.$, Eluens Pentan/Diethylether $\left.(10: 1), R_{\mathrm{f}}=0.36\right]$ ergab 2.26 g (55\%) 99 als farbloses Öl. - IR (Film): $v=3027 \mathrm{~cm}^{-1}, 2857,2221,1700,1652$, 1495, 1453, 1358, 1099, 936, 737, 698. - ${ }^{1} \mathrm{H}-\mathrm{NMR}\left(250 \mathrm{MHz}, \mathrm{CDCl}_{3}\right): \delta=0.71-1.00(\mathrm{~m}$, $6 \mathrm{H}, \mathrm{cPr}-\mathrm{H}), 1.30-1.42(\mathrm{~m}, 2 \mathrm{H}, \mathrm{cPr}-\mathrm{H}), 1.43-1.58$ (m, 1 H, cPr-H), 3.36 (dd, ${ }^{2} J=1$, $\left.{ }^{3} J=6 \mathrm{~Hz}, 2 \mathrm{H}, \mathrm{OCH}_{2}\right), 4.52\left(\mathrm{~s}, 2 \mathrm{H}, \mathrm{PhCH}_{2}\right), 5.83(\mathrm{~s}, 2 \mathrm{H}, \mathrm{CH}), 7.33(\mathrm{~m}, 5 \mathrm{H}, \mathrm{Ph}-\mathrm{H}) .-{ }^{13} \mathrm{C}-$ NMR (62.9 MHz, $\mathrm{CDCl}_{3}$, zusätzl. DEPT): $\delta=0.4$ (-, cPr-C), 6.1 (-, cPr-C), 8.7 (+, cPr-C), 13.6 (+, cPr-C), 22.1 (-, cPr-C), $72.1\left(+, \mathrm{CH}_{2}\right), 72.6\left(+, \mathrm{CH}_{2}\right), 74.5\left(\mathrm{C}_{\text {quart }}, \mathrm{C} \equiv \mathrm{C}\right), 75.4\left(\mathrm{C}_{\text {quart }}\right.$, $\mathrm{C} \equiv \mathrm{C}), 96.7\left(\mathrm{C}_{\text {quar, }}, \mathrm{C} \equiv \mathrm{C}\right), 98.3\left(\mathrm{C}_{\text {quart }}, \mathrm{C} \equiv \mathrm{C}\right), 120.0(-, \mathrm{CH}), 120.3 .(-, \mathrm{CH}), 127.6$ (-, Ph-C,

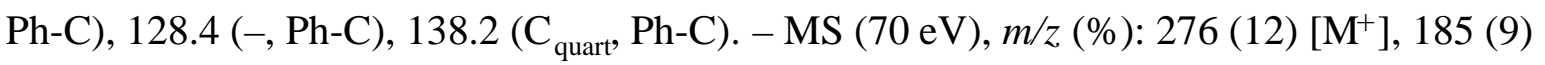
$\left[\mathrm{M}^{+}-\mathrm{PhCH}_{2}\right], 91(100)\left[\mathrm{PhCH}_{2}\right]$. 


\subsubsection{Trien- und Diendiine}

(E,E)-1-tert-Butoxy-dec-2,6-diin-4,8-

dien-10-ol (100): Nach AAV2 wurden

$39.0 \mathrm{mg}(5.7 \mathrm{~mol} \%)$ Bis-(triphenylphos-

phin)-palladium(II)-chlorid, $2.00 \mathrm{ml}$

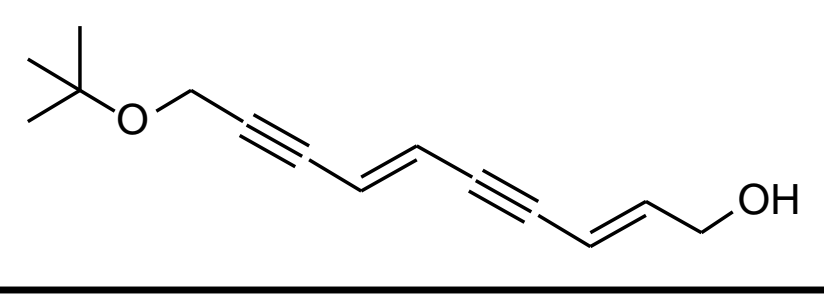

(20.2 mmol) Piperidin, $168 \mathrm{mg}$

(0.97 mmol) 92 und $202 \mathrm{mg}(2.46 \mathrm{mmol}) 23$ bei $0{ }^{\circ} \mathrm{C}$ mit $15.0 \mathrm{mg}$ (8.1mol\%) Kupfer(I)iodid 105 min zur Reaktion gebracht. Die säulenchromatographische Reinigung an Kieselgel [2.0 × $15 \mathrm{~cm}$, Eluens Pentan/Diethylether (1 : 1), $\left.R_{\mathrm{f}}=0.37\right]$ ergab $163 \mathrm{mg}(82 \%) 100 \mathrm{als}$ farblose Kristalle, Schmp. $28{ }^{\circ} \mathrm{C} .-{ }^{1} \mathrm{H}-\mathrm{NMR}\left(250 \mathrm{MHz}, \mathrm{CDCl}_{3}\right): \delta=1.25\left(\mathrm{~s}, 9 \mathrm{H}, \mathrm{CH}_{3}\right)$, 2.36 (br. s, $1 \mathrm{H}, \mathrm{OH}), 4.21$ (s, $4 \mathrm{H}, \mathrm{CH}_{2}$ ), 5.80-5.91 (m, $\left.1 \mathrm{H}, \mathrm{CH}\right), 5.92-6.13$ (m, $2 \mathrm{H}, \mathrm{CH}$ ), 6.20-6.31 (1 H, CH). $-{ }^{13} \mathrm{C}-\mathrm{NMR}\left(62.9 \mathrm{MHz}, \mathrm{CDCl}_{3}\right.$, zusätzl. DEPT): $\delta=27.3\left(+, \mathrm{CH}_{3}\right)$, $51.0\left(-, \mathrm{CH}_{2}\right), 62.6\left(-, \mathrm{CH}_{2}\right), 74.5\left[\mathrm{C}_{\text {quart }}, C\left(\mathrm{CH}_{3}\right)_{3}\right], 83.2\left(\mathrm{C}_{\text {quart }}, \mathrm{C} \equiv \mathrm{C}\right), 88.3\left(\mathrm{C}_{\text {quart }}, \mathrm{C} \equiv \mathrm{C}\right)$, $92.2\left(\mathrm{C}_{\text {quart }}, \mathrm{C} \equiv \mathrm{C}\right), 92.5\left(\mathrm{C}_{\text {quar, }}, \mathrm{C} \equiv \mathrm{C}\right), 109.6(+, \mathrm{CH}), 120.2(+, \mathrm{CH}), 121.0(+, \mathrm{CH}), 142.8(+$, $\mathrm{CH}) .-\mathrm{MS}(\mathrm{DCI}, 70 \mathrm{eV}), \mathrm{m} / z(\%): 454$ (3) $\left[2 \mathrm{M}^{+}+\mathrm{NH}_{4}^{+}\right], 253(18)\left[\mathrm{M}^{+}+\mathrm{NH}_{4}^{+}+\mathrm{NH}_{3}\right]$, $236(100)\left[\mathrm{M}^{+}+\mathrm{NH}_{4}^{+}\right], 219(7)\left[\mathrm{M}^{+}+\mathrm{H}^{+}\right]$.

(E,E)-1-tert-Butyldimethylsiloxydec-2,6-diin-4,8-dien-10-ol (101): Nach AAV2 wurden $431 \mathrm{mg}$ (4.4mol\%) Bis-(triphenylphosphin)-

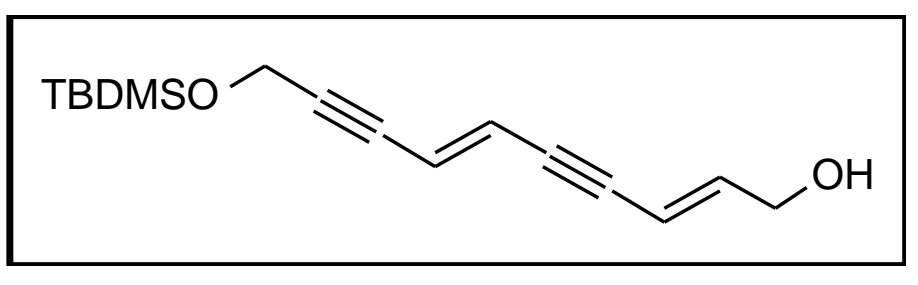

palladium(II)-chlorid, $20.0 \mathrm{ml}$ (202 mmol) Piperidin, $3.22 \mathrm{~g}$ (14.0 mmol) 93 und $1.38 \mathrm{~g}$ (16.8 mmol) 23 bei $0{ }^{\circ} \mathrm{C}$ mit $266 \mathrm{mg}(10 \mathrm{~mol} \%)$ Kupfer(I)-iodid $1.5 \mathrm{~h}$ zur Reaktion gebracht. Die säulenchromatographische Reinigung an Kieselgel $[3.0 \times 20 \mathrm{~cm}$, Eluens Pentan/Diethylether $(1: 1), R_{\mathrm{f}}=0.32 \mathrm{]}$ ergab $3.68 \mathrm{~g}(95 \%) 101$ als farbloses Ö1. $-{ }^{1} \mathrm{H}-\mathrm{NMR}(250 \mathrm{MHz}$, $\left.\mathrm{CDCl}_{3}\right): \delta=0.13\left(\mathrm{~s}, 6 \mathrm{H}, \mathrm{SiCH}_{3}\right), 0.92\left[\mathrm{~s}, 9 \mathrm{H}, \mathrm{C}\left(\mathrm{CH}_{3}\right)_{3}\right], 1.90-2.23$ (br. s, $\left.1 \mathrm{H}, \mathrm{OH}\right), 4.25$ $\left(\mathrm{d},{ }^{5} \mathrm{~J}=2 \mathrm{~Hz}, 2 \mathrm{H}, \mathrm{CH}_{2}\right), 4.46\left(\mathrm{~d},{ }^{5} \mathrm{~J}=2 \mathrm{~Hz}, 2 \mathrm{H}, \mathrm{CH}_{2}\right), 5.33-6.24(\mathrm{~m}, 3 \mathrm{H}, \mathrm{CH}), 6.28(\mathrm{dt}$, $\left.{ }^{3} J=13,{ }^{5} J=2 \mathrm{~Hz}, 1 \mathrm{H}, \mathrm{CH}\right) .-{ }^{13} \mathrm{C}-\mathrm{NMR}\left(62.9 \mathrm{MHz}, \mathrm{CDCl}_{3}\right.$, zusätzl. DEPT): $\delta=-5.2(+$, 
$\left.\mathrm{SiCH}_{3}\right), 18.3\left[\mathrm{C}_{\text {quart }}, C\left(\mathrm{CH}_{3}\right)_{3}\right], 25.8\left[+, \mathrm{C}\left(\mathrm{CH}_{3}\right)_{3}\right], 52.2\left(-, \mathrm{CH}_{2}\right), 62.8\left(-, \mathrm{CH}_{2}\right), 83.2\left(\mathrm{C}_{\text {quart }}\right.$, $\mathrm{C} \equiv \mathrm{C}), 88.4\left(\mathrm{C}_{\text {quart }}, \mathrm{C} \equiv \mathrm{C}\right), 92.4\left(\mathrm{C}_{\text {quart }}, \mathrm{C} \equiv \mathrm{C}\right), 93.2\left(\mathrm{C}_{\text {quart }}, \mathrm{C} \equiv \mathrm{C}\right), 109.8(+, \mathrm{CH}), 120.3(+, \mathrm{CH})$, $121.0(+, \mathrm{CH}), 142.6(+, \mathrm{CH})$.

(E,E,E)-1-tert-Butyldimethylsiloxydodeca-4,8-diin-2,6,10trien-12-ol (102): Nach AAV2 wurden $65.0 \mathrm{mg}$

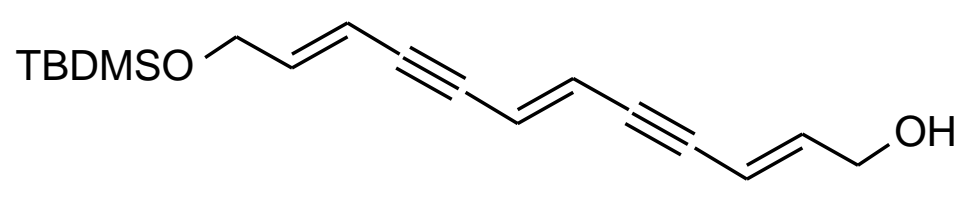

(5.8mol\%) Bis-(triphenylphosphin)-palladium(II)-chlorid, $3.00 \mathrm{ml}$ (30.3 mmol) Piperidin, $0.41 \mathrm{~g}(1.60 \mathrm{mmol}) 97$ und $0.28 \mathrm{~g}(3.4 \mathrm{mmol}) 23$ bei $0{ }^{\circ} \mathrm{C}$ mit $38.0 \mathrm{mg}(12 \mathrm{~mol} \%)$ Kupfer(I)iodid 1.5 h zur Reaktion gebracht. Die säulenchromatographische Reinigung an Kieselgel [2.0 $\times 15 \mathrm{~cm}$, Eluens Pentan/Diethylether (1 : 1), $\left.R_{\mathrm{f}}=0.63\right]$ ergab $0.33 \mathrm{~g}(68 \%) 102$ als farbloses Ö1. $-{ }^{1} \mathrm{H}-\mathrm{NMR}\left(250 \mathrm{MHz}, \mathrm{CDCl}_{3}\right): \delta=0.07$ (s, $\left.6 \mathrm{H}, \mathrm{SiCH}_{3}\right), 0.88\left[\mathrm{~s}, 9 \mathrm{H}, \mathrm{C}\left(\mathrm{CH}_{3}\right)_{3}\right]$, 2.06 (br. s, $1 \mathrm{H}, \mathrm{OH}), 4.14-4.26\left(\mathrm{~m}, 4 \mathrm{H}, \mathrm{CH}_{2}\right)$, 5.82-5.96 (m, $\left.2 \mathrm{H}, \mathrm{CH}\right), 6.02-6.10$ (m, $2 \mathrm{H}$, $\mathrm{CH}), 6.15-6.30(\mathrm{~m}, 2 \mathrm{H}, \mathrm{CH}) .-{ }^{13} \mathrm{C}-\mathrm{NMR}\left(62.9 \mathrm{MHz}, \mathrm{CDCl}_{3}\right): \delta=-5.4\left(\mathrm{SiCH}_{3}\right), 18.3$ $\left[C\left(\mathrm{CH}_{3}\right)_{3}\right], 25.8\left[\mathrm{C}\left(\mathrm{CH}_{3}\right)_{3}\right], 62.7\left(\mathrm{CH}_{2}\right), 62.8\left(\mathrm{CH}_{2}\right), 88.4(\mathrm{C} \equiv \mathrm{C}), 88.8(\mathrm{C} \equiv \mathrm{C}), 92.6(\mathrm{C} \equiv \mathrm{C})$, $93.4(\mathrm{C} \equiv \mathrm{C}), 108.4(\mathrm{CH}), 109.9(\mathrm{CH}), 120.1(\mathrm{CH}), 120.8(\mathrm{CH}), 142.5(\mathrm{CH}), 143.3(\mathrm{CH}) .-$ $\mathrm{C}_{18} \mathrm{H}_{26} \mathrm{O}_{2} \mathrm{Si}$ (302.49): ber. C 71.47, H 8.66, gef. C 71.59, H 8.48.

\subsection{Cyclopropanierungen an mehrfach ungesättigten Allylalkoholen}

(E)-1-tert-Butoxy-7-hydroxymethylcyclopropyl-hept-2,6-in-4-en (103): $\mathrm{Zu}$ einer Lösung von $0.60 \mathrm{ml}$ (5.85 mmol) Diethylzink, $0.40 \mathrm{ml}$ $(4.93 \mathrm{mmol}) \quad \mathrm{THF}$ und $0.96 \mathrm{ml}$

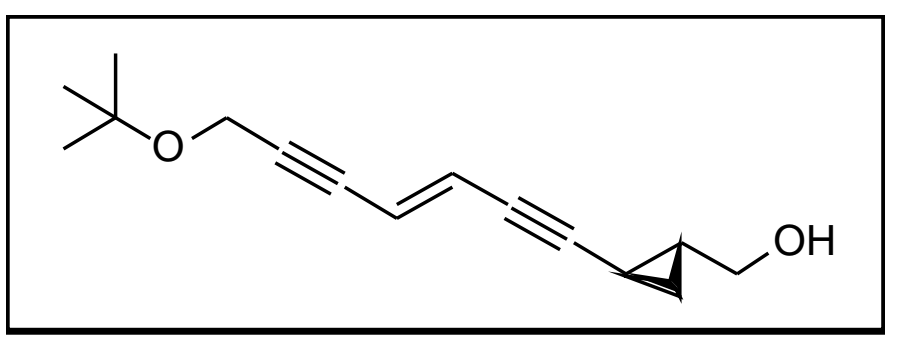

(11.9 mmol) Diiodmethan in $2 \mathrm{ml}$ Dichlormethan wurde bei $0{ }^{\circ} \mathrm{C}$ eine Lösung von $0.57 \mathrm{~g}$ (2.79 mmol) 100 in Dichlormethan zugetropft und $3.5 \mathrm{~h}$ bei derselben Temperatur gerührt. Zur Reaktionsmischung wurde $5 \mathrm{ml}$ ges. Ammoniumchloridlösung gegeben, der Niederschlag abfiltriert und mit $20 \mathrm{ml}$ Diethylether gewaschen. Die organische Phase wurde abge- 
trennt, über Magnesiumsulfat getrocknet und das Lösungsmittel i. Vak. entfernt. Die säulenchromatographische Reinigung an Kieselgel [2.0 $\times 15 \mathrm{~cm}$, Eluens Pentan/Diethylether (1:1), $\left.R_{\mathrm{f}}=0.45\right]$ ergab $315 \mathrm{mg}(54 \%) 103$ als gelbes Ö1. $-{ }^{1} \mathrm{H}-\mathrm{NMR}(250 \mathrm{MHz}$, $\left.\mathrm{CDCl}_{3}\right): \delta=0.77-0.91(\mathrm{~m}, 1 \mathrm{H}, \mathrm{cPr}-\mathrm{H}), 0.93-0.98(\mathrm{~m}, 1 \mathrm{H}, \mathrm{cPr}-\mathrm{H}), 1.22\left(\mathrm{~s}, 9 \mathrm{H} \mathrm{CH}_{3}\right), 1.25-$ 1.32 (m, $1 \mathrm{H}, \mathrm{cPr}-\mathrm{H}), 1.43-1.48$ (m, $1 \mathrm{H}, \mathrm{cPr}-\mathrm{H}), 2.02$ (br. s, $1 \mathrm{H}, \mathrm{OH}), 3.41-3.58$ (m, $2 \mathrm{H}$, $\left.\mathrm{CH}_{2} \mathrm{OH}\right), 4.19\left(\mathrm{~d},{ }^{5} \mathrm{~J}=1 \mathrm{~Hz}, 2 \mathrm{H}, \mathrm{COCH}_{2}\right), 5.89(\mathrm{~m}, 2 \mathrm{H}, \mathrm{CH}) .-{ }^{13} \mathrm{C}-\mathrm{NMR}(62.9 \mathrm{MHz}$, $\mathrm{CDCl}_{3}$, zusätzl. DEPT): $\delta=5.7$ (+, cPr-C), 13.3 (-, cPr-C), 24.5 (+, cPr-C), $27.3[+$, $\left.\mathrm{C}\left(\mathrm{CH}_{3}\right)_{3}\right], 50.9\left(-, \mathrm{CH}_{2}\right), 65.0\left(-, \mathrm{CH}_{2}\right), 74.4\left[\mathrm{C}_{\text {quar, }}, C\left(\mathrm{CH}_{3}\right)_{3}{ }^{*}\right], 75.1\left(\mathrm{C}_{\text {quart }}, \mathrm{C} \equiv \mathrm{C}^{*}\right), 83.2$ $\left(\mathrm{C}_{\text {quart }}, \mathrm{C} \equiv \mathrm{C}\right), 91.1\left(\mathrm{C}_{\text {quart }}, \mathrm{C} \equiv \mathrm{C}\right), 97.4\left(\mathrm{C}_{\text {quart }}, \mathrm{C} \equiv \mathrm{C}\right), 119.3(+, \mathrm{CH}), 121.4(+, \mathrm{CH})$.

(E)-1-tert-Butyldimethylsiloxy-7-hydroxymethylcyclopropyl-hept-2,6-in4-en (104): $\mathrm{Zu}$ einer Lösung von $1.50 \mathrm{ml}$ (14.6 mmol) Diethylzink,

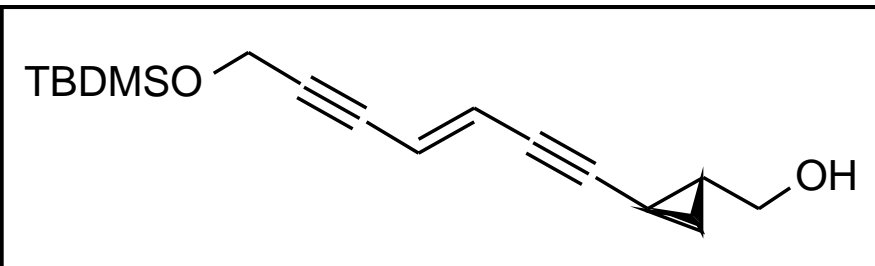
$1.20 \mathrm{ml}(14.8 \mathrm{mmol}) \mathrm{THF}$ und $2.40 \mathrm{ml}(29.8 \mathrm{mmol})$ Diiodmethan in $10 \mathrm{ml}$ Dichlormethan wurde bei $0{ }^{\circ} \mathrm{C} 1.08 \mathrm{~g}$ (3.91 mmol) 101 zugetropft. Man ließ $4 \mathrm{~h}$ bei Raumtemp. rühren und gab dann $10 \mathrm{ml}$ ges. Ammoniumchloridlösung dazu. Es wurde mit Diethylether $(3 \times 25 \mathrm{ml})$ extrahiert, mit ges. Natriumchloridlösung gewaschen, über Magnsiumsulfat getrocknet und das Lösungsmittel i. Vak. entfernt. Säulenchromatographie an Kieselgel $[3.0 \times 30 \mathrm{~cm}$, Eluens Pentan/Diethylether (2: 1), $R_{\mathrm{f}}=0.23$ ] ergab $0.57 \mathrm{~g}(50 \%) 104$ als gelbes Öl. $-{ }^{1} \mathrm{H}-\mathrm{NMR}$ $\left(250 \mathrm{MHz}, \mathrm{CDCl}_{3}\right): \delta=0.11\left(\mathrm{~s}, 6 \mathrm{H}, \mathrm{SiCH}_{3}\right), 0.82-1.03\left[\mathrm{~m}, 11 \mathrm{H}, \mathrm{C}\left(\mathrm{CH}_{3}\right), \mathrm{cPr}-\mathrm{H}\right] 1.27-1.35$ (m, $1 \mathrm{H}, \mathrm{cPr}-\mathrm{H}), 1.40-1.52(\mathrm{~m}, 1 \mathrm{H}, \mathrm{cPr}-\mathrm{H}), 3.43-3.62\left(\mathrm{~m}, 2 \mathrm{H}, \mathrm{CH}_{2} \mathrm{OH}\right), 4.43$ (s, $2 \mathrm{H}$, $\left.\mathrm{SiOCH}_{2}\right), 5.91(\mathrm{~s}, 2 \mathrm{H}, \mathrm{CH}) .-{ }^{13} \mathrm{C}-\mathrm{NMR}\left(62.9 \mathrm{MHz}, \mathrm{CDCl}_{3}\right.$, zusätzl. DEPT): $\delta=-5.2(-$, $\mathrm{SiC}), 5.8$ (-,, cPr-C), 13.3 (+, cPr-C), $18.3\left[\mathrm{C}_{\text {quart }}, C\left(\mathrm{CH}_{3}\right)_{3}\right], 24.6$ (-, cPr-C), 25.8 [-, $\left.\mathrm{C}\left(\mathrm{CH}_{3}\right)_{3}\right], 52.1\left(+, \mathrm{CH}_{2}\right), 65.1\left(+, \mathrm{CH}_{2}\right), 75.1\left(\mathrm{C}_{\text {quart }}, \mathrm{C} \equiv \mathrm{C}\right), 83.2\left(\mathrm{C}_{\text {quart }}, \mathrm{C} \equiv \mathrm{C}\right), 92.1\left(\mathrm{C}_{\text {quart }}\right.$, $\mathrm{C} \equiv \mathrm{C}), 97.5\left(\mathrm{C}_{\text {quart }}, \mathrm{C} \equiv \mathrm{C}\right), 119.3(-, \mathrm{CH}), 121.4(-, \mathrm{CH}) .-\mathrm{MS}(70 \mathrm{eV}), \mathrm{m} / z(\%): 290(3)\left[\mathrm{M}^{+}\right]$, 233 (10), 203 (8), 75 (100). 
(E,E)-1-tert-Butyldimethylsi-

loxy-9-hydroxymethylcyclo-

propyl-non-4,8-in-2,6-en

(105): Zu einer Lösung von

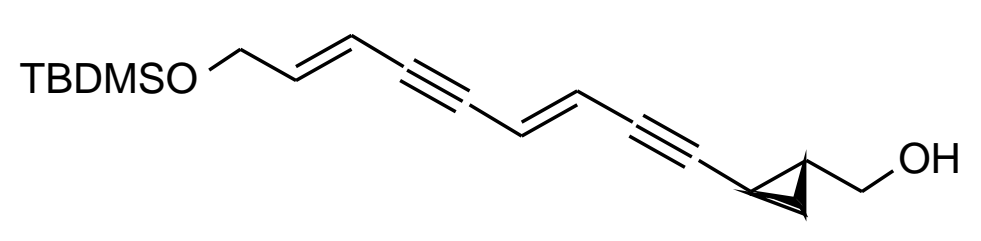

$0.52 \mathrm{ml}$ (5.07 mmol) Diethylzink, $0.50 \mathrm{ml}$ (6.16 mmol) THF und 0.80 ml (9.93 mmol) Diiodmethan in $2 \mathrm{ml}$ Dichlormethan wurde bei $0{ }^{\circ} \mathrm{C}$ eine Lösung von $220 \mathrm{mg}(0.70 \mathrm{mmol}) 102$ in $1 \mathrm{ml}$ Dichlormethan zugetropft und $3.5 \mathrm{~h}$ bei derselben Temperatur gerührt. Man gab $5 \mathrm{ml}$ ges. Ammoniumchloridlösung zur Reaktionsmischung, extrahierte mit Diethylether $(3 \times 10 \mathrm{ml})$ trocknete über Magnesiumsulfat und entfernte das Lösungsmittel i. Vak. Säulenchromatographie an Kieselgel [2.0 $\times 20 \mathrm{~cm}$, Eluens Pentan/Diethylether $\left.(1: 1), R_{\mathrm{f}}=0.36\right]$ ergab $57.0 \mathrm{mg}(30 \%) 105$ als gelbes Öl. $-{ }^{1} \mathrm{H}-\mathrm{NMR}\left(250 \mathrm{MHz}, \mathrm{CDCl}_{3}\right): \delta=0.05(\mathrm{~s}, 6 \mathrm{H}$, $\left.\mathrm{SiCH}_{3}\right), 0.53-0.64$ (m, $\left.2 \mathrm{H}, \mathrm{cPr}-\mathrm{H}\right), 0.86$ [s, $\left.9 \mathrm{H}, \mathrm{C}\left(\mathrm{CH}_{3}\right)\right], 0.91-1.05$ (m, $\left.2 \mathrm{H}, \mathrm{cPr}-\mathrm{H}\right), 2.23$ (br. s, $1 \mathrm{H}, \mathrm{OH}), 3.42-3.58\left(\mathrm{~m}, 4 \mathrm{H}, \mathrm{CH}_{2}\right), 5.87-6.24(\mathrm{~m}, 4 \mathrm{H}, \mathrm{CH}) .-{ }^{13} \mathrm{C}-\mathrm{NMR}(62.9 \mathrm{MHz}$, $\left.\mathrm{CDCl}_{3}\right): \delta=-3.6\left(\mathrm{SiCH}_{3}\right), 4.8(\mathrm{cPr}-\mathrm{C}), 12.9$ (cPr-C), $15.2(\mathrm{cPr}-\mathrm{C}), 18.2\left[\mathrm{C}\left(\mathrm{CH}_{3}\right)_{3}\right], 26.6$ $\left[\mathrm{C}\left(\mathrm{CH}_{3}\right)_{3}\right], 62.7\left(\mathrm{CH}_{2}\right), 65.8\left(\mathrm{CH}_{2}\right), 109.7(\mathrm{C} \equiv \mathrm{C}), 110.2(\mathrm{C} \equiv \mathrm{C}), 112.6(\mathrm{C} \equiv \mathrm{C}), 119.2(\mathrm{C} \equiv \mathrm{C})$, $120.1(\mathrm{CH}), 121.3(\mathrm{CH}), 142.1(\mathrm{CH}), 157.8(\mathrm{CH})$. 


\subsection{Oxidationen an Endiinen}

\subsubsection{Dihydroxylierungen nach Sharpless}

Allgemeine Arbeitsvorschrift zur Dihydroxylierung von Alkenen nach Sharpless (AAV3): Um ein Millimol Alken umzusetzen, wurde eine Mischung aus veränderlichen Anteilen Kaliumosmat Dihydrat, (DHQD) 2 PHAL, 0.94 g (3.00 mmol) Kaliumhexacyanoferrat(III), $0.41 \mathrm{~g}$ (3.00 mmol) Kaliumcarbonat bei $0{ }^{\circ} \mathrm{C}$ oder Raumtemp. in $5 \mathrm{ml}$ Wasser und $5 \mathrm{ml}$ tertButanol gelöst, das Alken und veränderliche Aquivalente Methansulfonsäureamid zugegeben und die Mischung mehrere Stunden intensiv gerührt. Dann wurde bei $0{ }^{\circ} \mathrm{C}$ langsam $0.50 \mathrm{~g}$ (3.96 mmol) Natriumsulfit zur Reaktionsmischung gegeben, auf Raumtemp. erwärmt und 30 min weitergerührt. Man extrahierte mit Dichlormethan $(3 \times 15 \mathrm{ml})$, wusch die vereinigten organischen Extrakte mit $5 \mathrm{ml} 1 \mathrm{~N}$ Kaliumhydroxidlösung, $5 \mathrm{ml}$ ges. Natriumchloridlösung, trocknete über Magnesiumsulfat und entfernte das Lösungsmittel i. Vak. Säulenchromatographie an Kieselgel (Eluens Diethylether) ergab die Diole.

(3R,4R)-1,6-Bis(tert-Butyl)hex-1,5-diin-2,3-diol

(116): Nach AAV3 wurde $189 \mathrm{mg}$ (1.00 mmol) $88 \mathrm{mit}$ $16.0 \mathrm{mg}$ (4.34mol\%) Kaliumosmat Dihydrat, $79 \mathrm{mg}$ (10.1 mol\%) (DHQD) 2 PHAL und $476 \mathrm{mg}$ (5.00 mmol) Methansulfonsäureamid $24 \mathrm{~h}$ bei Raumtemp. gerührt. Säulenchromatographie an Kieselgel $(2.0 \times 10 \mathrm{~cm}$,

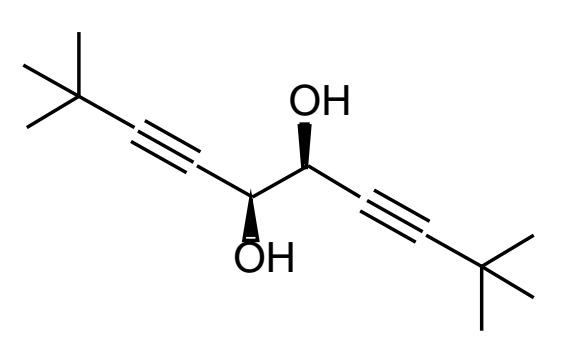

$\left.R_{\mathrm{f}}=0.38\right)$ ergab $109 \mathrm{mg}(49 \%) 116$ als weißen Feststoff (Schmp. $\left.68^{\circ} \mathrm{C}\right)$ und $80 \mathrm{mg}(42 \%)$ Edukt. $-{ }^{1} \mathrm{H}-\mathrm{NMR}\left(250 \mathrm{MHz}, \mathrm{CDCl}_{3}\right): \delta=1.24$ [s, $\left.18 \mathrm{H}, \mathrm{C}\left(\mathrm{CH}_{3}\right)_{3}\right], 2.70$ (br. s, $2 \mathrm{H}, \mathrm{OH}$ ), $4.32(\mathrm{~s}, 2 \mathrm{H}, \mathrm{CH}) .-{ }^{13} \mathrm{C}-\mathrm{NMR}\left(62.9 \mathrm{MHz}, \mathrm{CDCl}_{3}\right.$, zusätzl. DEPT): $\delta=27.3\left[\mathrm{C}_{\text {quart }}\right.$, $\left.C\left(\mathrm{CH}_{3}\right)_{3}\right], 30.8\left[+, \mathrm{C}\left(\mathrm{CH}_{3}\right)_{3}\right], 66.5(+, \mathrm{CH}), 75.5\left(\mathrm{C}_{\text {quart }}, \mathrm{C} \equiv \mathrm{C}\right), 95.5\left(\mathrm{C}_{\text {quart }}, \mathrm{C} \equiv \mathrm{C}\right)$. 
(3R,4R)-1,6-Bis(Cyclopropyl)hex-1,5-in-2,3-ol

(119): Nach AAV3 wurde $156 \mathrm{mg}(1.00 \mathrm{mmol}) 98$ mit $3.60 \mathrm{mg}(0.98 \mathrm{~mol} \%)$ Kaliumosmat Dihydrat, $15.2 \mathrm{mg}(1.95 \mathrm{~mol} \%)$ (DHQD) ${ }_{2} \mathrm{PHAL}$ und $380 \mathrm{mg}$ (3.99 mmol) Methansulfonsäureamid $24 \mathrm{~h}$ bei Raum-

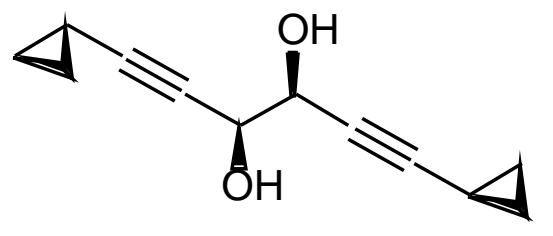

temp. gerührt. Säulenchromatographie an Kieselgel $\left(2.0 \times 10 \mathrm{~cm}, R_{\mathrm{f}}=0.29\right)$ ergab $70.0 \mathrm{mg}$ $(37 \%) 119$ als weißen Feststoff (Schmp. $59^{\circ} \mathrm{C},[\alpha]_{\mathrm{D}}{ }^{22}=-120.21\left(\mathrm{c}=3.000, \mathrm{CH}_{2} \mathrm{Cl}_{2}\right)$ ) und $80.0 \mathrm{mg}(51 \%)$ Edukt. $-{ }^{1} \mathrm{H}-\mathrm{NMR}\left(250 \mathrm{MHz}, \mathrm{CDCl}_{3}\right): \delta=0.62-0.83(\mathrm{~m}, 8 \mathrm{H}, \mathrm{cPr}-\mathrm{H}), 1.08-$ 1.26 (m, $2 \mathrm{H}, \mathrm{cPr}-\mathrm{H}), 3.23$ (s, $2 \mathrm{H}, \mathrm{OH}), 4.21$ (s, $2 \mathrm{H}, \mathrm{CH}) .-{ }^{13} \mathrm{C}-\mathrm{NMR}\left(62.9 \mathrm{MHz}, \mathrm{CDCl}_{3}\right.$, zusätzl. DEPT): $\delta=-0.7$ (+, cPr-C), $8.1(-, \mathrm{cPr}-\mathrm{C}), 66.3(+, \mathrm{CH}), 72.3\left(\mathrm{C}_{\text {quart }}, \mathrm{C} \equiv \mathrm{C}\right), 90.3$ $\left(\mathrm{C}_{\text {quart }}, \mathrm{C} \equiv \mathrm{C}\right) .-\mathrm{MS}(70 \mathrm{eV}), \mathrm{m} / z(\%): 190(1)\left[\mathrm{M}^{+}\right], 161(35), 95(92),\left[\mathrm{M}^{+}-\mathrm{C}_{8} \mathrm{H}_{7} \mathrm{O}\right], 41$ (100) $\left[\mathrm{C}_{3} \mathrm{H}_{5}{ }^{+}\right]$.

(3R,4R)-1-(Benzyloxymethylcyclopropyl)-6cyclopropylhex-1,5-diin-2,3-diol

(120): Nach AAV3 wurde 266 mg (0.96 mmol) 99 mit $3.60 \mathrm{mg}(1.02 \mathrm{~mol} \%)$ Kaliumosmat Dihydrat, $15.6 \mathrm{mg}(2.09 \mathrm{~mol} \%)(\mathrm{DHQD})_{2} \mathrm{PHAL}$

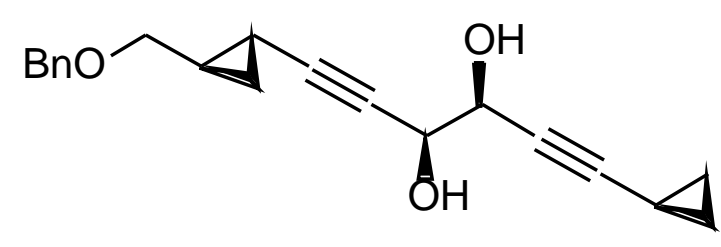
und $380 \mathrm{mg}$ (4.16 mmol) Methansulfonsäureamid 24 h bei Raumtemp. gerührt. Säulenchromatographie an Kieselgel $\left(2.0 \times 10 \mathrm{~cm}, R_{\mathrm{f}}=0.18\right)$ ergab $60.0 \mathrm{mg}(20 \%) 120$ als farbloses Öl und 96.0 mg (35\%) Edukt. - IR (Film): $v=3388 \mathrm{~cm}^{-1}, 2862,2240,1717,1453,1070$, 738, 699. $-{ }^{1} \mathrm{H}-\mathrm{NMR}\left(250 \mathrm{MHz}, \mathrm{CDCl}_{3}\right): \delta=0.63-0.82(\mathrm{~m}, 5 \mathrm{H}, \mathrm{cPr}-\mathrm{H}), 0.83-0.99(1 \mathrm{H}$, cPr-H), 1.06-1.26 (m, 2 H, cPr-H), 1.27-1.47 (1 H, cPr-H), 2.96 (br. s, 2 H, OH), 3.22-3.40 $\left(\mathrm{m}, 2 \mathrm{H}, \mathrm{OCH}_{2}\right), 4.23$ (s, $\left.2 \mathrm{H}, \mathrm{CH}\right), 4.45$ (s, $\left.2 \mathrm{H}, \mathrm{PhCH}_{2}\right), 7.18-7.29$ (m, $\left.5 \mathrm{H}, \mathrm{Ph}-\mathrm{H}\right) .-{ }^{13} \mathrm{C}-$ NMR (62.9 MHz, $\mathrm{CDCl}_{3}$, zusätzl. DEPT): $\delta=-0.6$ (+, cPr-C), 5.2 (+, Cpr-C), 8.3 (-, cPrC), 13.1 (-, cPr-C), 21.5 (+, cPr-C), $66.3(+, \mathrm{CHOH}), 72.0\left(-, \mathrm{OCH}_{2}\right), 72.3\left(\mathrm{C}_{\text {quart }}, \mathrm{C} \equiv \mathrm{C}\right), 72.5$ $\left(-, \mathrm{PhCH}_{2}\right), 73.3\left(\mathrm{C}_{\text {quart }}, \mathrm{C} \equiv \mathrm{C}\right), 89.0\left(\mathrm{C}_{\text {quart }}, \mathrm{C} \equiv \mathrm{C}\right), 90.5\left(\mathrm{C}_{\text {quart }}, \mathrm{C} \equiv \mathrm{C}\right), 127.6(+, \mathrm{Ph}-\mathrm{C}), 128.3$ (+, Ph-C), $137.9\left(\mathrm{C}_{\text {quart }}\right.$ Ph-C). - MS (DCI, $\left.70 \mathrm{eV}\right), \mathrm{m} / \mathrm{z}(\%): 328(100)\left[\mathrm{M}^{+}+\mathrm{NH}_{4}^{+}\right]$. 
Versuch zur Darstellung von (4R,5R)-1-tert-Butyldimethylsiloxy-7-hydroxymethylcyclopropyl-hept-4-en-2,6-in (160): Nach AAV3 wurde $290 \mathrm{mg}$ (1.00 mmol) $101 \mathrm{mit} 18.4 \mathrm{mg}$ (4.99mol\%) Kaliumosmat Dihydrat, $37.0 \mathrm{mg}$ (4.75mol\%) (DHQD) ${ }_{2}$ PHAL und $475 \mathrm{mg}$ (4.99 mmol) Methansulfonsäureamid 24 h bei Raumtemp. gerührt. Es konnten nur $160 \mathrm{mg}$ (55\%) Edukt zurückgewonnen werden.

Versuch zur Darstellung von (4R,5R)-1-tert-Butyloxy-7-hydroxymethylcyclopropyl-hept-4en-2,6-in (161): Nach AAV3 wurde $50.0 \mathrm{mg}$ (0.22 mmol) $100 \mathrm{mit} 2.0 \mathrm{mg}$ (2.58mol\%) Kaliumosmat Dihydrat, $12.0 \mathrm{mg}(7.34 \mathrm{~mol} \%)$ (DHQD) ${ }_{2}$ PHAL und $95.0 \mathrm{mg}(4.76 \mathrm{mmol}) \mathrm{Me}-$ thansulfonsäureamid $24 \mathrm{~h}$ bei $50{ }^{\circ} \mathrm{C}$ gerührt. Das NMR-Spektrum des Rohprodukts zeigte nur noch Zersetzungsprodukte.

\subsubsection{Dihydroxylierungen mit Rutheniumtetroxid}

Allgemeine Arbeitsvorschrift zur Dihydroxylierung von Alkenen mit Rutheniumtetroxid (AAV4): $\mathrm{Zu}$ einer auf $0{ }^{\circ} \mathrm{C}$ gekühlten Lösung von $1 \mathrm{mmol}$ des Alkens in $6 \mathrm{ml}$ Acetonitril und 6 ml Essigsäureethylester gibt man auf ein Mal eine Lösung von 14.5 mg (7.00 mol\%) Rutheniumtrichlorid Trihydrat und $312 \mathrm{mg}$ (1.50 mmol) Natriumperiodat. Nach 3 min intensivem Rühren gibt man $10 \mathrm{ml}$ ges. Natriumthiosulfatlösung zu. Man extrahiert mit Essigsäureethylester $(3 \times 15 \mathrm{ml})$, trocknet über Magnesiumsulfat und entfernt das Lösungsmittel i. Vak. Säulenchromatographie an Kieselgel ergibt die sauberen Diole.

1,6-Bis(tert-Butyloxymethyl)hex-1,5-diin-2,3-diol (131): Nach AAV4 wurde $188 \mathrm{mg}$ (1.00 mmol) 116 umgesetzt. Säulenchromatographie an Kieselgel $(2 \times 15 \mathrm{~cm}$, Eluens Diethylether, $\left.R_{\mathrm{f}}=0.38\right)$ ergab $143 \mathrm{mg}(64 \%) 131$ und $12.0 \mathrm{mg}(6 \%)$ Edukt. Die Spektren waren mit der nach AAV3 hergestellten Verbindung identisch. 
1,6-Bis(Cyclopropyl)hex-1,5-in-2,3-ol (133): Nach AAV4 wurde 156 mg (1.00 mmol) 119 umgesetzt. Säulenchromatographie an Kieselgel $\left(2 \times 20 \mathrm{~cm}\right.$, Eluens Diethylether, $\left.R_{\mathrm{f}}=0.29\right)$ ergab 143 mg (64\%) 133 und 12.0 mg (6\%) Edukt. Die Spektren waren mit der nach AAV3 hergestellten Verbindung identisch.

1-(Benzyloxymethylcyclopropyl)-6-cyclopropylhex-1,5-in-2,3-ol (134): Nach AAV4 wurde $250 \mathrm{mg}$ (0.90 mmol) 120 umgesetzt. Säulenchromatographie an Kieselgel [2 × $15 \mathrm{~cm}$, Eluens Pentan/Diethylether (1:1), $\left.R_{\mathrm{f}}=0.09\right)$ ergab $79.0 \mathrm{mg}(28 \%) 134$ und $49.0 \mathrm{mg}(20 \%)$ Edukt. Die Spektren waren mit der nach AAV3 hergestellten Verbindung identisch.

1-tert-Butyldimethylsiloxy-7-hydroxymethylcyclopropyl-2,6-in-4,5-ol

(132): Nach AAV4 wurde $301 \mathrm{mg}$ (1.04 mmol) $11712 \mathrm{~min}$ bei $0{ }^{\circ} \mathrm{C}$

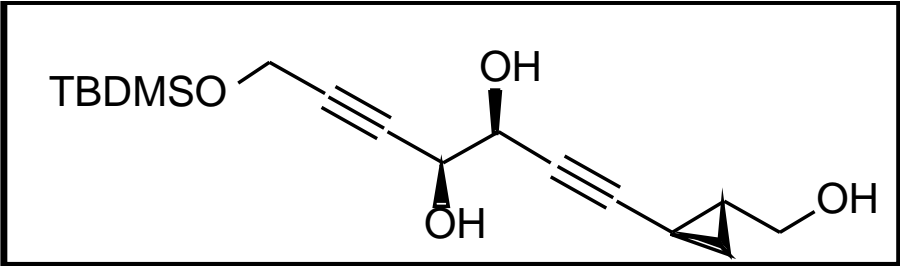
umgesetzt. Säulenchromatographie an Kieselgel $\left(2 \times 15 \mathrm{~cm}\right.$, Eluens Diethylether, $\left.R_{\mathrm{f}}=0.40\right)$ ergab $78.0 \mathrm{mg}$ (23\%) 132 und $122 \mathrm{mg}$ (42\%) Edukt. $-{ }^{1} \mathrm{H}-\mathrm{NMR}\left(250 \mathrm{MHz}, \mathrm{CDCl}_{3}\right)$ : $\delta=0.14\left(\mathrm{~s}, 6 \mathrm{H}, \mathrm{SiCH}_{3}\right), 0.69-0.78(\mathrm{~m}, 1 \mathrm{H}, \mathrm{cPr}-\mathrm{H}), 0.91$ [s, $\left.9 \mathrm{H}, \mathrm{C}\left(\mathrm{CH}_{3}\right)_{3}\right], 1.21-1.39(\mathrm{~m}$, $2 \mathrm{H}, \mathrm{cPr}-\mathrm{H}), 1.42-1.51$ (m, $1 \mathrm{H}, \mathrm{cPr}-\mathrm{H}), 3.22-3.34$ (m, $2 \mathrm{H}, \mathrm{CH}), 3.60-3.82$ (br. s, $3 \mathrm{H}, \mathrm{OH}$ ), $4.44\left(\mathrm{~s}, 4 \mathrm{H}, \mathrm{CH}_{2}\right) .-{ }^{13} \mathrm{C}-\mathrm{NMR}\left(62.9 \mathrm{MHz} \mathrm{CDCl}_{3}\right.$, zusätzl. DEPT): $\delta=-5.2\left(-, \mathrm{SiCH}_{3}\right), 5.3$ (-, cPr-C), 12.8 (+, cPr-C), $18.3\left[\mathrm{C}_{\text {quart }}, C\left(\mathrm{CH}_{3}\right)_{3}\right], 24.0(-, \mathrm{cPr}-\mathrm{C}), 25.8\left[-, \mathrm{C}\left(\mathrm{CH}_{3}\right)_{3}\right], 51.8$ $\left(+, \mathrm{CH}_{2}\right), 65.1\left(+, \mathrm{CH}_{2}\right), 65.9(-, \mathrm{CH}), 66.1(-, \mathrm{CH}), 73.4\left(\mathrm{C}_{\text {quart }}, \mathrm{C} \equiv \mathrm{C}\right), 82.3\left(\mathrm{C}_{\text {quart }}, \mathrm{C} \equiv \mathrm{C}\right)$, $84.7\left(\mathrm{C}_{\text {quart }}, \mathrm{C} \equiv \mathrm{C}\right), 89.2\left(\mathrm{C}_{\text {quart }}, \mathrm{C} \equiv \mathrm{C}\right)$.

\subsubsection{Dihydroxylierungen mit Kaliumpermanganat}

Versuch zur Darstellung von 1,6-Bis(Cyclopropyl)hex-1,5-in-2,3-ol (119): Zu einer Lösung von $156 \mathrm{mg}$ (1.00 mmol) 98 in $3 \mathrm{ml}$ Dichlormethan wurden $3 \mathrm{ml}$ einer Lösung von $166 \mathrm{mg}$ (1.00 mmol) Tetraethylammoniumchlorid Hydrat in 40\%iger Natriumhydroxidlösung zugegeben. Dann gab man $158 \mathrm{mg}(1.00 \mathrm{mmol})$ Kaliumpermanganat dazu und rührte $20 \mathrm{~h}$ bei 
Raumtemp. Die Mischung wurde über eine Säule filtriert (Eluens Diethylether), die von unten nach oben $5 \mathrm{~cm}$ Kieselgel und $5 \mathrm{~cm}$ Celite enthielt. Es konnten nur $137 \mathrm{mg}$ (88\%) Edukt zurückgewonnen werden.

Versuch zur Darstellung von 1,6-Bis(Cyclopropyl)hex-1,5-in-2,3-ol (119): Eine Lösung von $156 \mathrm{mg}(1.00 \mathrm{mmol}) 98$ und $64.2 \mathrm{mg}(20 \mathrm{~mol} \%)$ Tetrabutylammoniumbromid wurde auf $0{ }^{\circ} \mathrm{C}$ gekühlt und $15 \mathrm{ml}$ einer Lösung von $474 \mathrm{mg}(3.00 \mathrm{mmol})$ Kaliumpermanganat in 20\%iger Natriumhydroxidlösung zugetropft. Es wurde $18 \mathrm{~h}$ bei Raumtemp. gerührt, dann kühlte man die Mischung auf $0{ }^{\circ} \mathrm{C}$ und tropfte $15 \mathrm{ml}$ einer ges. Lösung Natriumsulfit zu. Die Mischung wurde mit konz. Salzsäure auf pH 7 eingestellt und mit Dichlormethan $(3 \times 15 \mathrm{ml})$ extrahiert. Man trocknete über Magnesiumsulfat und entfernte das Lösungsmittel i. Vak. Es konnte kein Produkt isoliert werden.

\subsubsection{Epoxidierungen}

Versuch zur Darstellung von 1-tert-Butoxy-7-hydroxymethylcyclopropyl-hept-4,5-epoxy2,6-in (162): Zu einer Lösung von 100 mg (0.43 mmol) 116 in 15 ml Dichlormethan wurden bei $0{ }^{\circ} \mathrm{C} 230 \mathrm{mg}$ (1.62 mmol) Dinatriumhydrogenphosphat und $247 \mathrm{mg}$ (1.43 mmol) 3Chlorperoxybenzoesäure gegeben und 25 h bei Raumtemp. gerührt. Man gab 15 ml 10\%ige Natriumsulfitlösung zur Reaktionsmischung, wusch mit ges. Natriumchloridlösung und entfernte das Lösungsmittel i. Vak. Im NMR-Spektrum des Rohprodukts konnte kein Produkt nachgewiesen werden.

Versuch zur Darstellung von 1,6-Bis(Cyclopropyl)hex-2,3-epoxy-1,5-in (163): Zu einer Lösung von $156 \mathrm{mg}(1.00 \mathrm{mmol}) 119$ in $15 \mathrm{ml}$ Dichlormethan wurde bei $0{ }^{\circ} \mathrm{C} 1.14 \mathrm{~g}$ (8.03 mmol) Dinatriumhydrogenphosphat und $493 \mathrm{mg}$ (2.86 mmol) 3-Chlorperoxybenzoesäure gegeben und 20 h bei Raumtemp. gerührt. Man gab 20 ml 10\%ige Natriumsulfitlösung 
zur Reaktionsmischung, wusch mit ges. Natriumchloridlösung und entfernte das Lösungsmittel i. Vak. Im NMR-Spektrum des Rohprodukts konnte kein Produkt nachgewiesen werden. 


\subsection{Transformationen an dihydroxylierten Endiinen}

(3R,4R)-1,6-Bis(cyclopropyl)hexa-1,5-dien-3,4-diol

(147): Es wurden $2.00 \mathrm{ml}$ (7.00 mmol) einer 3.5 M Lösung von Natrium-bis-(2-methoxyethoxy)-dihydroaluminat (Red-Al) in Diethylether gegeben und die

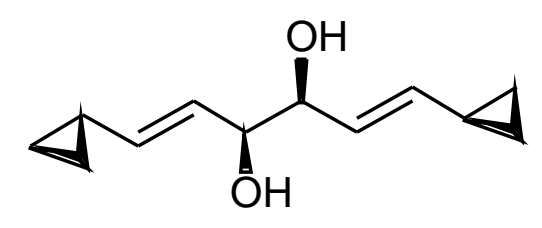
Mischung auf $0{ }^{\circ} \mathrm{C}$ gekühlt. Dann tropfte man eine Lösung von $204 \mathrm{mg}$ (1.07 mmol) $119 \mathrm{in}$ $2 \mathrm{ml}$ Diethylether zu, ließ auf Raumtemp. erwärmen und rührte 9 h weiter. Die Reaktionsmischung wurde auf $0{ }^{\circ} \mathrm{C}$ gekühlt, langsam $10 \mathrm{ml}$ 10\%ige Schwefelsäure zugetropft und so lange gerührt, bis sich der Niederschlag aufgelöst hatte. Man extrahierte mit Essigsäureethylester $(3 \times 15 \mathrm{ml})$, wusch mit $5 \mathrm{ml}$ ges. Natriumhydrogencarbonatlösung, $10 \mathrm{ml}$ ges. Natriumchloridlösung und trocknete über Magnesiumsulfat. Das Lösungsmittel wurde i. Vak. entfernt und der Rückstand an Kieselgel $(3.0 \times 30 \mathrm{~cm}$, Eluens Diethylether, $\left.R_{\mathrm{f}}=0.59\right)$ gereinigt. Man erhielt $160 \mathrm{mg}(77 \%) 147$ als farbloses Öl, $[\alpha]_{\mathrm{D}}{ }^{22}=-165.33$ $\left(\mathrm{c}=2.530, \mathrm{CH}_{2} \mathrm{Cl}_{2}\right) .-{ }^{1} \mathrm{H}-\mathrm{NMR}\left(250 \mathrm{MHz}, \mathrm{CDCl}_{3}\right): \delta=0.30-0.36(\mathrm{~m}, 4 \mathrm{H}, \mathrm{cPr}-\mathrm{H}), 0.63-$ 0.70 (m, 4 H, cPr-H), 1.29-1.38 (m, 2 H, cPr-H), 2.33 (br. s, 2 H, OH), 3.81-3.83 (m, 2 H, $\mathrm{CHOH}), 5.20\left(\mathrm{dd},{ }^{3} J=9,{ }^{3} J=15 \mathrm{~Hz}, 2 \mathrm{H}, \mathrm{CH}\right), 5.46\left(\mathrm{dd},{ }^{3} J=6,{ }^{3} J=15 \mathrm{~Hz}, 2 \mathrm{H}, \mathrm{CH}\right) .-$ ${ }^{13} \mathrm{C}-\mathrm{NMR}\left(62.9 \mathrm{MHz}, \mathrm{CDCl}_{3}\right.$, zusätzl. DEPT): $\delta=6.6$ (-, cPr-C), 6.8 (-, cPr-C), $13.5(+$, cPr-C), 75.6 (+, CHOH), 125.8 (+, CH), 137.8 (+, CH). - MS (70 eV), m/z (\%): 194 (12) $\left[\mathrm{M}^{+}\right], 177(10)\left[\mathrm{M}^{+}-\mathrm{OH}\right], 147$ (18), 105 (22), 97 (100) $\left[\mathrm{M}^{+}-\mathrm{C}_{6} \mathrm{H}_{9} \mathrm{O}\right], 79$ (32).

(3R,4R,5R,6R,7R,8R)-1,2-Bis(bicyclopropyl)ethan-1,2ol (148): $\mathrm{Zu}$ einer Lösung von $1.03 \mathrm{ml}$ (10.1 mmol) Diethylzink, $0.80 \mathrm{ml}(9.86 \mathrm{mmol})$ THF und $1.61 \mathrm{ml}$ (20.0 mmol) Diiodmethan in $5 \mathrm{ml}$ Dichlormethan wur-

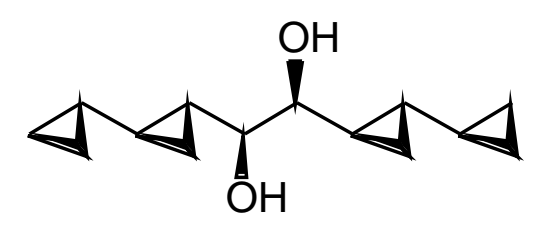
den bei $0{ }^{\circ} \mathrm{C}$ eine Lösung von $165 \mathrm{mg}$ (0.85 mmol) 147 in $2 \mathrm{ml}$ Dichlormethan getropft. Man ließ auf Raumtemp. erwärmen und rührte 16 h weiter. Dann wurde die Reaktionsmischung auf $20 \mathrm{ml}$ ges. Ammoniumchloridlösung gegeben, mit Essigsäureethylester $(3 \times 20 \mathrm{ml})$ extrahiert und über Magnesiumsulfat getrocknet. Säulenchromatographie an Kieselgel $\left(2.0 \times 20 \mathrm{~cm}\right.$, Eluens Diethylether, $\left.R_{\mathrm{f}}=0.52\right)$ ergab $120 \mathrm{mg}(64 \%) 148$ als farbloses Öl. -

${ }^{1} \mathrm{H}-\mathrm{NMR}\left(250 \mathrm{MHz}, \mathrm{CDCl}_{3}\right): \delta=-0.05-0.09(\mathrm{~m}, 4 \mathrm{H}, \mathrm{cPr}-\mathrm{H}), 0.25-0.56(\mathrm{~m}, 8 \mathrm{H}, \mathrm{cPr}-\mathrm{H})$, 
0.76-0.97 (m, $6 \mathrm{H}, \mathrm{cPr}-\mathrm{H}), 1.50-2.13$ (br. s, $2 \mathrm{H}, \mathrm{OH}), 3.02$ (d, $\left.{ }^{3} \mathrm{~J}=4 \mathrm{~Hz}, \mathrm{CH}\right) .-{ }^{13} \mathrm{C}-\mathrm{NMR}$ (62.9 $\mathrm{MHz}^{\mathrm{CDCl}} \mathrm{CD}_{3}$, zusätzl. DEPT): $\delta=2.5$ (+, cPr-C), 3.2 (+, cPr-C), 7.0 (+, cPr-C), 11.7 (-, cPr-C), 18.1 (-, cPr-C), 20.3 (-, cPr-C), 77.7 (-, CH). - MS (DCI, $\left.\mathrm{NH}_{3}, 70 \mathrm{eV}\right), \mathrm{m} / z(\%)$ : $684(10)\left[3 \mathrm{M}+\mathrm{NH}_{4}^{+}\right], 462(100)\left[2 \mathrm{M}^{+} \mathrm{NH}_{4}^{+}\right], 257(57)\left[\mathrm{M}+\mathrm{NH}_{4}{ }^{+}+\mathrm{NH}_{3}\right], 240(77)$ $\left[\mathrm{M}+\mathrm{NH}_{4}^{+}\right]$.

$(3 R, 4 R, 5 R, 6 R, 7 R, 8 R)-(E)-1,2-B i s($ bicyclopropyl)-

ethen (148): $\mathrm{Zu}$ einer Lösung von $120 \mathrm{mg}(0.50 \mathrm{mmol})$ 147 in $4 \mathrm{ml}$ wasserfreiem Dichlormethan wurden bei

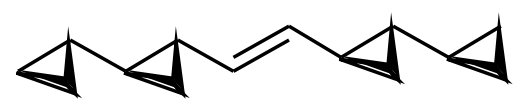

$0{ }^{\circ} \mathrm{C} 640 \mathrm{mg}$ (5.24 mmol) 4-Dimethylaminopyridin gegeben und $0.25 \mathrm{ml}$ (2.79 mmol) einer 85\%igen Lösung von Thiophosgen in Tetrachlorkohlenstoff getropft und 20 min bei derselben Temperatur weitergerührt. Es wurde $1 \mathrm{~g}$ Kieselgel zur Reaktionsmischung gegeben und das Lösungsmittel i. Vak. entfernt. Säulenfiltration über Kieselgel [2.0 × 15 cm, Eluens Pentan/Diethylether (1:1)] ergaben 73 mg Rohprodukt 158, das ohne Aufarbeitung weiter umgesetzt wurde. $73 \mathrm{mg} 158$ und $0.30 \mathrm{ml} 157$ wurden $6 \mathrm{~h}$ bei $40^{\circ} \mathrm{C}$ gerührt. Die Reaktionsmischung wurde an Kieselgel chromatographiert $(2.0 \times 15 \mathrm{~cm}$, Eluens Pentan, $\left.R_{\mathrm{f}}=0.60\right)$ und man erhielt $25.0 \mathrm{mg}(26 \%) 148$ als farbloses Ö1. $-{ }^{1} \mathrm{H}-\mathrm{NMR}(250 \mathrm{MHz}$, $\left.\mathrm{CDCl}_{3}\right): \delta=0.01-0.06(\mathrm{~m}, 4 \mathrm{H}, \mathrm{cPr}-\mathrm{H}), 0.31-0.42$ (m, $\left.8 \mathrm{H}, \mathrm{cPr}-\mathrm{H}\right), 0.78-0.84$ (m, $2 \mathrm{H}, \mathrm{cPr}-$ H), $1.03-1.18(\mathrm{~m}, 2 \mathrm{H}, \mathrm{cPr}-\mathrm{H}), 1.25-1.31(4 \mathrm{H}, \mathrm{cPr}-\mathrm{H}), 5.02\left(\mathrm{dd},{ }^{3} J=5,{ }^{4} J=3 \mathrm{~Hz}, 2 \mathrm{H}\right.$, $\mathrm{CH}) .-{ }^{13} \mathrm{C}-\mathrm{NMR}\left(62.9 \mathrm{MHz}, \mathrm{CDCl}_{3}\right.$, zusätzl. DEPT): $\delta=2.3(-, \mathrm{cPr}-\mathrm{C}), 3.3$ (-, cPr-C), 11.2 (-, cPr-C), 11.9 (+, cPr-C), 14.1 (+, cPr-C), 19.8 (+, cPr-C), 130.8 (+, CH). - MS $(70 \mathrm{eV}), m / z(\%): 188(10)\left[\mathrm{M}^{+}\right], 105(53), 91(100), 79(100), 41(51)\left[\mathrm{C}_{3} \mathrm{H}_{4}{ }^{+}\right] .-\mathrm{C}_{14} \mathrm{H}_{20}$ : 188.1565 (korrekte HRMS). 


\section{Zusammenfassung}

In dieser Arbeit wurde der Zugang zu Oligocyclopropylen über 1,6-substituierte trans-Endiine 19 untersucht. Hierzu mußten zuerst Alkine als geeignete Kupplungspartner synthetisiert werden.

Die Synthese von trans-1-Ethinyl-2-benzyloxymethylcyclopropan (53) als Kupplungspartner zur Darstellung der Endiine 19 gelang in einer sechsstufigen Synthese in zufriedenstellenden 31\% Gesamtausbeute. Die Synthese von 1-Ethinyl-2-hydroxymethylcyclopropan (65) über die Simmons-Smith-Reaktion war nur in mäßigen 46\% Ausbeute unter aufwendigen Reaktionsbedingungen möglich. Das als Vorläufer gewählte 23 ließ sich auch in den geschützten Formen $\mathbf{6 3}$ und $\mathbf{6 4}$ nicht effizient zu entsprechenden Cyclopropanen umsetzen.

Die Kupplung zu den 1,6-substituierten trans-Endiinen 19 gelang dagegen in guten bis sehr guten Ausbeuten sowohl in einer einstufigen Synthese zu symmetrischen, als auch in einer zweistufigen Synthese zu unsymmetrische Endiine. Dabei konnte eine große Anzahl unterschiedlicher Alkine umgesetzt werden, über die der Zugang zu drei bis fünf Cyclopropylringe enthaltende Ketten möglich wäre. Endständige Alkoholgruppen sollten dabei geschützt werden, da besonders die Kupplungen zum Enin 94 eine schlechtere Ausbeute zeigte. Eine Eintopfvariante zur Herstellung von unsymmetrischen Endiinen mißlang, obwohl sie in der Literatur mit guten Ausbeuten beschrieben ist.

Cyclopropanringe sollten schon vor der Kupplung zum Endiin im Alkin vorhanden sein. Die Cyclopropanierungen an mehrfach ungesättigten Allylalkohlen 82 verliefen mit weniger guten Ausbeuten.

Sehr problematisch war die 1,2-Dihydroxylierung der Endiine $19 \mathrm{zu}$ Diindiolen 20. Hierbei gelang nur die Dihydroxylierung nach Sharpless und mit Rutheniumtetroxid. Die Umsetzungen nach Sharpless mußten bei Raumtemperatur über $24 \mathrm{~h}$ mit größeren Anteilen an Osmium und Ligand durchgeführt werden, als im käuflichen AD-mix enthalten sind, um Ausbeuten von 18-49\% zu erreichen. Die Reaktion kam in allen Fällen zum Stillstand und eine Verlän- 
gerung der Reaktionszeit hatte keinen Effekt auf die Ausbeuten. Dieser Reaktionsschritt stellt, bezogen auf die vorhandenen Substanzmengen, ein "Nadelöhr" für die weitere Synthese dar. Mit Rutheniumtetroxid lagen die Ausbeuten an Diindiolen 20 im allgemeinen etwas höher, aber dafür waren die Mengen an zurückgewonnenem Endiin geringer. Andere Oxidationsmethoden mit Kaliumpermanganat oder Epoxidierungen an Endiinen scheiterten.

Die weiteren Umsetzungen an Dioldiinen verliefen relativ problemlos. Die Reduktion an Dioldiinen 20 verlief am besten mit Red-Al. Eine doppelte Cyclopropanierung an $147 \mathrm{zu}$ 148 konnte erfolgreich durchgeführt werden und die Corey-Winter-Eliminierung führte zu 159. Wegen Substanzmangel konnte die letzte Cyclopropanierung nicht mehr durchgeführt werden. In der Literatur sind jedoch ähnliche Umsetzungen beschrieben, sodaß sich die Synthese voraussichtlich zu Ende führen läßt. 


\section{E. Literatur}

[1] J. Salalün, Curr. Med. Chem.,1995, 2, 511-542.

[2] L. Grombie, M. Eliott, Progr. Chem. Org. Nat. Prod., 1961, 19, 121-125.

[3] A. Nuhn, Chemie der Naturstoffe, Bd. 2, Hirzel-Verlag, Stuttgart 1990, 480-488.

${ }^{[4]}{ }^{[4 a]}$ D. E. Minnikin, N. Polgar, J. Chem. Soc. J. Chem. Soc., Chem. Comm., 1967, 20,

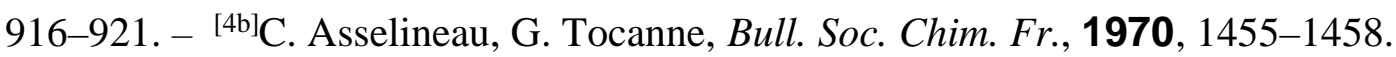

[5] M. Yoshida, M. Hashimoto, M. Yamashita, N. Shigematsu, M. Okuhara, M. Kohsaka, K. Horikoshi, J. Antibiotics, 1990, 748-754.

[6] M. S. Kuo, J. I. Cialdella, C. K. Marschke, M. J. Dupuis, G. P. Li, D. A. Kloosterman, C. H. Spilman, V. P. Marshall, J. Am. Chem. Soc., 1995, 117, 10629-10634.

${ }^{[7]} \quad{ }^{[7 \mathrm{a}]}$ C. R. Theberge, C. K. Zercher, Tett. Lett., 1994, 35, 9181-9184. - ${ }^{[7 b]}$ A. G. M. Barrett, K. Kasdorf, D. J. Williams, J. Chem. Soc., Chem. Comm., 1994, 1781-1782. ${ }^{[7 c]}$ A. G. M. Barrett, W. W. Doubleday, G. J. Tustin, A. J. P. White, D. J. Williams, J.

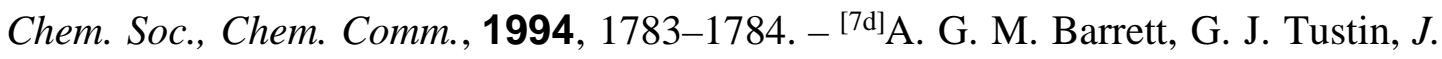
Chem. Soc., Chem. Comm., 1995, 355-356. - $\left.{ }^{[7 e}\right]$ A. G. M. Barrett, K. Kasdorf, A. J. P. White, D. J. Williams, J. Chem. Soc., Chem. Comm., 1995, 649-650. - ${ }^{[7 f]}$ A.G.M. Barrett, W. W. Doubleday, K. Kasdorf, G. J. Tustin, A. J. P. White, D. J. Williams, J. Chem. Soc., Chem. Comm., 1995, ${ }^{[7 \mathrm{~g}]} \mathrm{R}$. W. Armstrong, K. W. Maurer, Tetrahedron Lett., 1995, 36, 357-360. - ${ }^{[7 h]}$ J. R. Falck, B. Mekonnen, J. Yu, J.-Y. Lai, J. Am Chem. Soc., 1996, 118, 6096-6097.

${ }^{[8]}{ }^{[8 a]}$ A. G. M. Barrett, K. Kasdorf, G. J. Tustin, D. J. Williams,J. Chem. Soc., Chem. Comm., 1995, 1143-1144. - ${ }^{[8 b]}$ A. G. M. Barrett, K. Kasdorf, J. Chem. Soc., Chem. Comm., 1996, 325-326. - ${ }^{[8 c]}$ A. G. M. Barrett, W. W. Doubleday, K. Kasdorf, G. J. Tu-

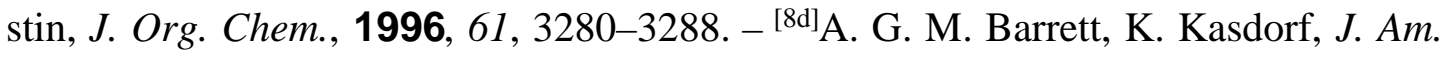
Chem. Soc., 1996, 118, 11030-11037.

[9] A. G. M. Barrett, D. Hamprecht, A. J. P. White, D. J. William, J. Am. Chem. Soc., 1996, $118,7863-7864$.

${ }^{[10]}{ }^{[10 a]}$ A. B. Charette, H. Juteau, J. Am. Chem. Soc., 1994, 116, 2651-2652. - ${ }^{[10 b]}$ A. B. Charette, S. Prescott, C. Brochu, J. Org. Chem., 1995, 60, 1081-1083. - ${ }^{[10 c]}$ A. B. Charette, H. Juteau, H. Lebel, D. Deschenes, Tetrahedron Lett., 1996, 37, 7925-7928. 
${ }^{[10 d] M}$. D. Turnbull, J. Chem. Soc, Perkin Trans, 1997, 1241-1247.

[11] ${ }^{[11 a]}$ M. von Seebach, Diplomarbeit, Universität Göttingen, 1997. - ${ }^{[11 b]}$ M. von Seebach, geplante Dissertation, Universität Göttingen.

[12] A. de Meijere, S. I. Kozhushkov, T. Spaeth, N. S. Zefirov, J. Org. Chem., 1993, 58, 502.

${ }^{[13] \quad[13 a] S . ~ L o ̈ h r, ~ D i p l o m a r b e i t, ~ U n i v e r s i t a ̈ t ~ G o ̈ t t i n g e n, ~ 1997 .-~}{ }^{[13 b]}$ S. Löhr, geplante Dissertation, Universität Göttingen.

${ }^{[14]}{ }^{[14 \mathrm{a}]} \mathrm{O} . \mathrm{W}$. Thiele, Lipide Isoprenoide mit Steroiden, Georg-Thieme-Verlag, Stuttgart, 1979, 17-87. - ${ }^{[14 b]}$ A. L. Leninger, Biochemie, VCH-Verlagsgesellschaft, Weinheim, 1987, 447-459.

[15] [15a]J. Furukawa, N. Kawabata, J. Nishimura, Tetrahedron, 1968, 24, 53-58. - ${ }^{[15 b]}$ J. Furukawa, N. Kawabata, J. Nishimura, Tetrahedron Lett., 1968, 3495-3498.

[16] K. B. Sharpless, H. C. Kolb, Chem. Rev., 1994, 94, 2483-2547.

${ }^{[17]}{ }^{[17 a]}$ L. L. McCoy, J. Am. Chem. Soc., 1958, 80, 6568-6572. - ${ }^{[17 b]}$ L. Skatteboel, Y. Stenstroem, Act. Chem. Scan., 1989, 43, 93-96.

[18] A. J. Mancuso, S. Huang, D. Swern, J. Org. Chem., 1978, 43, 2480-2482.

[19] [19a]J. Wolinsky, K. L. Erickson, J. Org. Chem., 1965, 30, 2208-2211. - ${ }^{[19 b]}$ S. Miyano, Y. Izumi, H. Hashimoto, J. Chem. Soc. Chem. Comm., 1978, 446-447. - ${ }^{[19 c]}$ M. Matsumoto, K. Kuroda, Tetrahedron Lett., 1980, 21, 4021-4024. - [19d]P. Pianetti, P. Rollin, J. R. Pougny, Tetrahedron Lett., 1986, 27, 5853-5856.

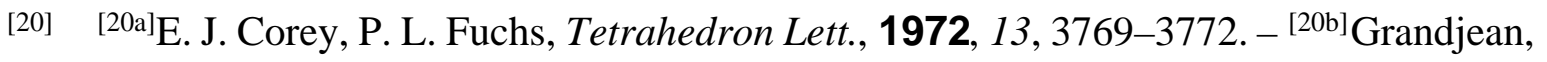
P. Pale, J. Chuche, Tetrahedron Lett., 1994, 35, 3529-3530.

[21] A. Bavvy, U. Lygo, Tetrahedron, 1988, 44, 6889-6894.

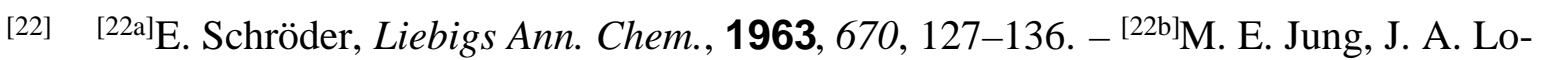
we, M. A. Lyster, M. Node, R. W. Pfluger, R. W. Brown, Tetrahedron, 1984, 40, 4751-4766.

[23] L. Brandsma, Preparative Acetylenic Chemistry, Elsevier Publishing Company, Amsterdam, London, New York, 1971, 63-64.

[24] ${ }^{[24 a]}$ H.E.Simmons, R. D. Smith, J. Am. Chem. Soc., 1958, 80, 5323-5326. - ${ }^{[24 b]}$ H. E. Simmons, T. L. Cairns, S. A. Vladuchick, M. C. Hoiness, Org. React., 1973, 20, 123. - ${ }^{[24 c]}$ J. Furukawa, N. Kawabata, J. Nishimura, Tetrahedron Lett., 1966, 33533355. 


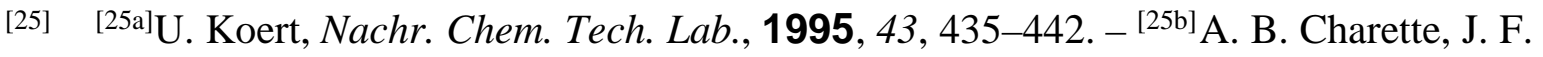
Marcoux, Synlett, 1995, 1197-1207.

[26] A. Alexakis, M. Gardette, S. Colin, Tetrahedron Lett., 1988, 29, 2951-2154.

[27] E. J. Corey, A. Venkateswarlu, J. Am Chem. Soc., 1972, 94, 6190-6192.

[28] J. M. Russo, W. A. Price, J. Org. Chem., 1993, 58, 3589-3590.

${ }^{[29]}{ }^{[29 a]} Y$. Ukaji, M. Nishimura, T. Fujisawa, Chem. Lett., 1992, 61-64. - ${ }^{[29 b]} Y$. Ukaji, K. Sada, K. Inomata, Chem. Lett., 1993, 1227-1230.

${ }^{[30]}{ }^{[30 a]}$ H. Takahashi, M. Yoshioka, M. Ohno, S. Kobayashi, Tetrahedron Lett., 1992, 33, 2575-2578. - ${ }^{[30 b]}$ N. Imai, K. Sakamoto, H. Takahashi, S. Kobayashi, Tetrahedron Lett., 1994, 35, 7045-7048. - ${ }^{[30 c]}$ S. E. Denmark, B. L. Christenson, D. M. Coe, S. P. O’Connor, Tetrahedron Lett., 1995, 36, 2215-2218. - ${ }^{[30 \mathrm{~d}]}$ S. E. Denmark, B. L. Christenson, S. P. O'Connor, Tetrahedron Lett., 1995, 36, 2219-2222.

[31] [31a]I. Arai, A. Mori, H. Yamamoto, J. Am. Chem. Soc., 1985, 107, 8254-8256. - ${ }^{[31 b]}$ A. Mori, I. Arai, H. Yamamoto, Tetrahedron, 1986, 42, 6447-6458.

[32] D. B. Dess, J. C. Martin, J. Org. Chem., 1983, 48, 4156-4158.

${ }^{[33]}{ }^{[33 a]}$ K. Sonogashira, Comprehensive Organic Synthesis, Pergamon Oxford, 1991, B. M. Trost, Ed., 521-549. - ${ }^{[33 b]}$ R. Rossi, A. Carpita, F. Bellina, Org. Prep. and Proced. Int., 1995, 27, 127-160.

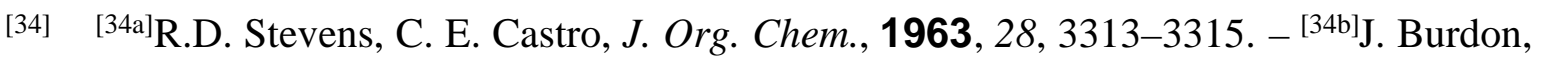
P. L. Coe, C. R. March, J. C. Tatlow, J. Chem. Soc., Chem. Comm., 1967, 1259-1258. ${ }^{[35]}{ }^{[35 a]}$ K. P. C. Vollhardt, L. S. Winn, Tetrahedron Lett., 1985, 26, 709-713. - ${ }^{[35 b]}$ J. Anthony, C. B. Knobler, F. Diederich, Angew. Chem., 1993, 437-440. - ${ }^{[35 c]}$ G. McGaffin, A. de Meijere, Synthesis, 1994, 35, 583-591.

[36] D. Chemin, G. Linstrumelle, Tetrahedron, 1994, 50, 5335-5344.

${ }^{[37]}{ }^{[37 a]}$ V. Ratovelomanana, G. Linstrumelle, Tetrahedron Lett., 1981, 22, 315-318. ${ }^{[37 b]}$ V. Ratavelomanana, G. Linstrumelle, Tetrahedron Lett., 1984, 25, 6001-6004. ${ }^{[37 \mathrm{c}]}$ D. Guillerm, G. Linstrumelle, Tetrahedron Lett., 1985, 26, 3811-3812. - ${ }^{[37 \mathrm{~d}]} \mathrm{Y}$. Lu, C. W. Harwig, A. G. Fallis, J. Org. Chem., 1993, 58, 4202-4204.

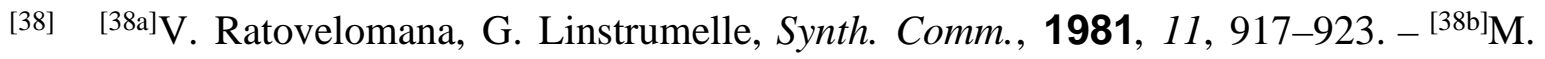
Alami, G. Instrumelle, Tetrahedron Lett., 1991, 32, 6109-6112. - ${ }^{[38 c]}$ R. H. Grupps, D. Kratz, Chem. Ber., 1993, 126, 149-157. - ${ }^{[38 d] J . ~ K a b b a r a, ~ C . ~ H o f f m a n n, ~ D . ~ S c h i n z e r, ~}$ Synthesis, 1995, 36, 299-302. 
Becker, M. A. Soler, K. B. Sharpless, Tetrahedron, 1995, 51, 1345-1376. - ${ }^{[40 c]}$ E. J. Corey, A. Guzman-Perez, J. Am. Chem. Soc., 1995, 117, 10805-10816. - ${ }^{[40 \mathrm{~d}] Z . ~ M . ~}$ Wang, K. B. Sharpless, J. Org. Chem., 1994, 59, 8302-8303. - ${ }^{[40 e] P . ~ G . ~ A n d e r s o n, ~ K . ~}$ B. Sharpless, J. Am. Chem. Soc., 1993, 115, 7047-7048.

[41] H. Nemoto, J. Miyata, H. Hakamata, K. Fukumoto, Tetrahedron Lett., 1995, 36, 10551058.

[42] K. S. Jeong, P. Sjö, K. B. Sharpless, Tetrahedron Lett., 1992, 33, 3833-3836.

[43] P. F. Shuda, M. B. Cichowicz, M. R. Heimann, Tetrahedron Lett., 1983, 24, 38293831.

[44] P. H. J. Carlsen, T. Katsuki, V. S. Martin, K. B. Sharpless, J. Org. Chem., 1981, 46, 3936-3939.

[45] S.-I. Murahashi, T. Saito, H. Hanaoka, Y. Murakami, T. Naota, H. Kumobayashi, S. Akutagawa, J. Org. Chem., 1993, 58, 2929-2932.

${ }^{[46]}{ }^{[46 a]}$ T. A. Foglia, P. A. Barr, A. J. Malloy, M. J. Costanzo, J. Am. Oil Chemist's Soc., 1977, 54, 870-872. - ${ }^{[46 b]}$ T. K. M. Shing, E. K. W. Tam, V. W.-F. Tai, I. H. F. Chung, Q. Jiang, Chem. Eur. J., 1996, 2, 50-57. - ${ }^{[46 c]}$ T. K. M. Shing, V. W.-F. Tai, E. K. W. Tam, Angew. Chem., 1994, 106, 2408-2409.

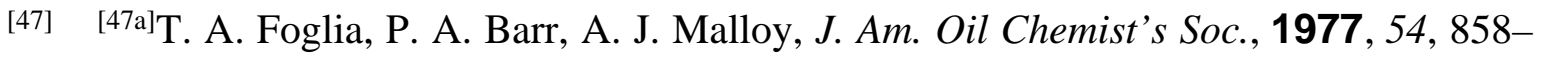
861. - ${ }^{[47 b]}$ T. Okimoto, D. Swern, J. Am. Oil Chemist's Soc., 1977, 54, 862-866. ${ }^{[47 c]}$ D. J. Sam, H. F. Simmons, J. Am. Chem. Soc., 1972, 94, 4024-4025. - ${ }^{[47 d] W . ~ P . ~}$ Weber, J. P. Shepherd, Tetrahedron Lett., 1972, 4907-4908. - ${ }^{[47 \mathrm{e}]}$ L. Mangoni, M. Adinolfi, G. Barone, M. Parilli, Tetrahedron Lett., 1973, 4485-4486. ${ }^{[47 f]}$ S. Wolfe, C. F. Ingold, J. Am. Chem. Soc., 1981, 103, 940-941.

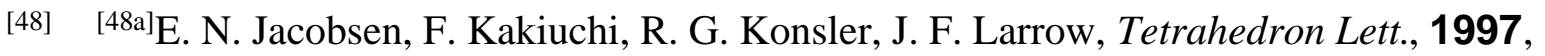
38, 773-776. - ${ }^{[48 b]}$ M. H. Wu, E. N. Jacobsen, Tetrahedron Lett., 1997, 38, $1693-$ 1696.

[49] ${ }^{[49 a]}$ M. H. Durand, Mem. Pres. Soc. Chim., 1961, 2387-2392. - ${ }^{[49 b] S . ~ H o l a n d, ~ R . ~}$ Epsztein, I. Marszak, Bull. Soc. Chim. Fr., 1969, 5, 3213-3219. - ${ }^{[49 c]}$ S. Galaj, Y. Guichon, Y.-L. Pascal, C. R. Acad. Sc. Paris, 1979, 541-543.

${ }^{[50]}{ }^{[50 a]}$ K. Nakasuji, S. Akiyama, M. Nakagawa, Bull. Chem. Soc. Jap., 1972, 45, 883- 
891. - C. Otte, Dissertation, Universität Göttingen, 1994.

${ }^{[51] \quad[51 a]}$ S. E. Denmark, T. K. Jones, J. Org. Chem., 1982, 47, 4595-4596. - ${ }^{[51 b]}$ R. E. Doolittle, Synthesis, 1984, 730-731.

${ }^{[52]}{ }^{[52 a]}$ E. J. Corey, A. Winter, J. Am. Chem. Soc., 1963, 85, 2677-2678. - ${ }^{[52 b]}$ E. J. Corey, Pure Appl. Chem., 1967, 14, 19-37. - ${ }^{[52 c]}$ E. J. Corey, F. Hopkins, Tetrahedron Lett., 1982, 23, 1979-1981. 


\section{F. Spektren}

${ }^{1} \mathrm{H}$ - und ${ }^{13} \mathrm{C}$-Spektren ausgewählter Verbindungen. 


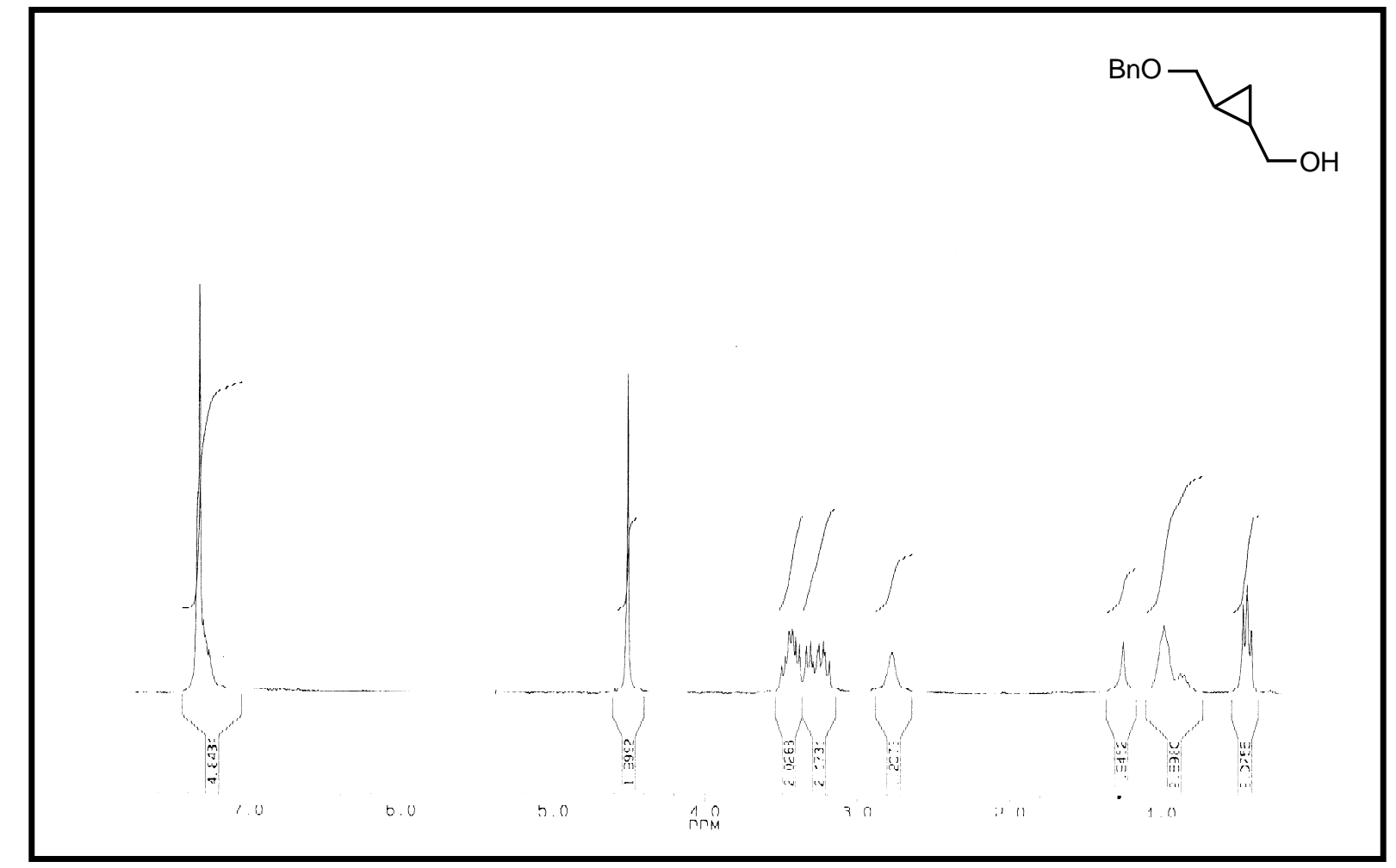

${ }^{1} H$-NMR-Spektrum von trans-1-Benzyloxymethyl-2-hydroxymethylcyclopropan (39)

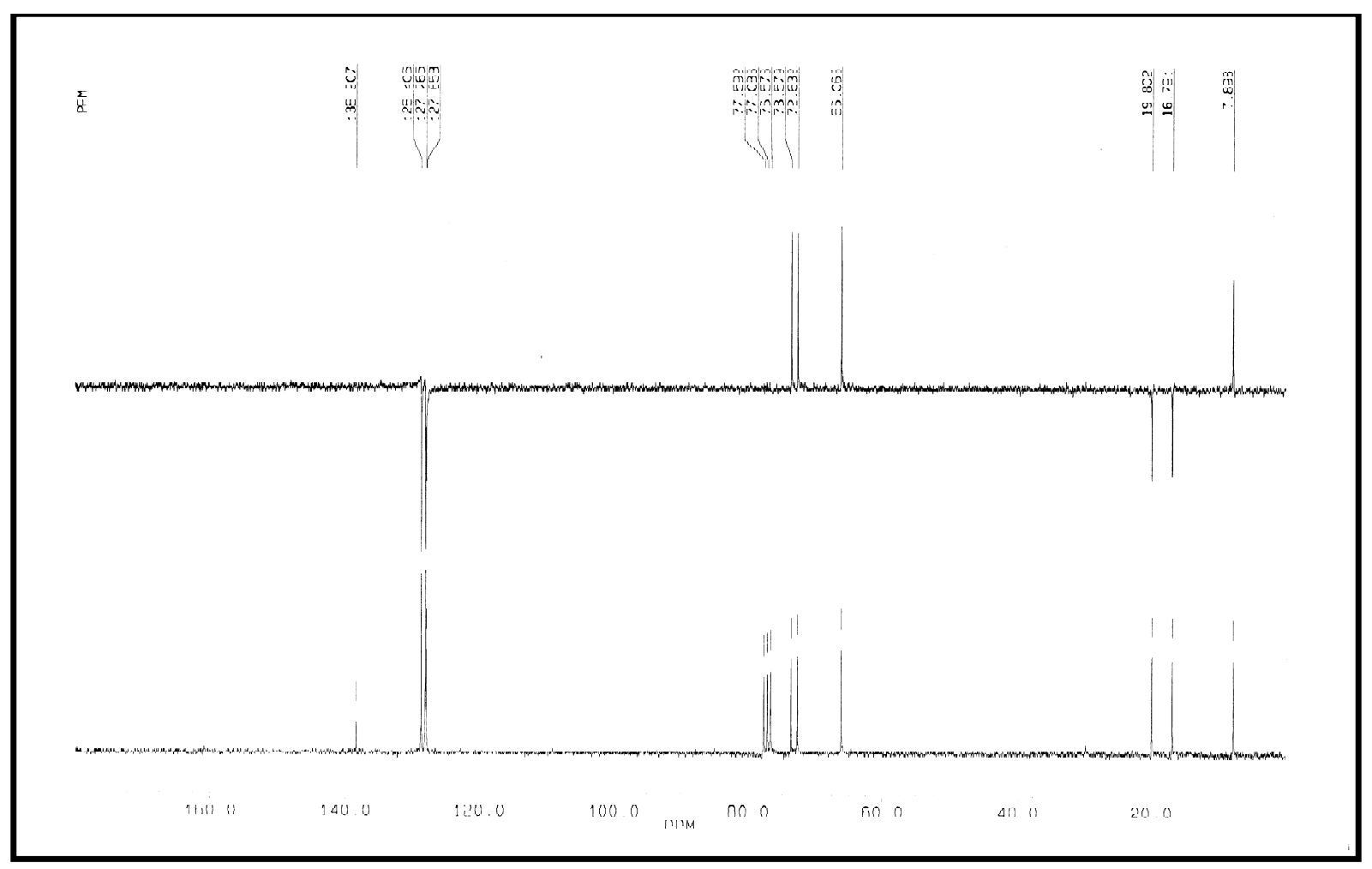

${ }^{13}$ C-NMR-Spektrum von trans-1-Benzyloxymethyl-2-hydroxymethylcyclopropan (39) 


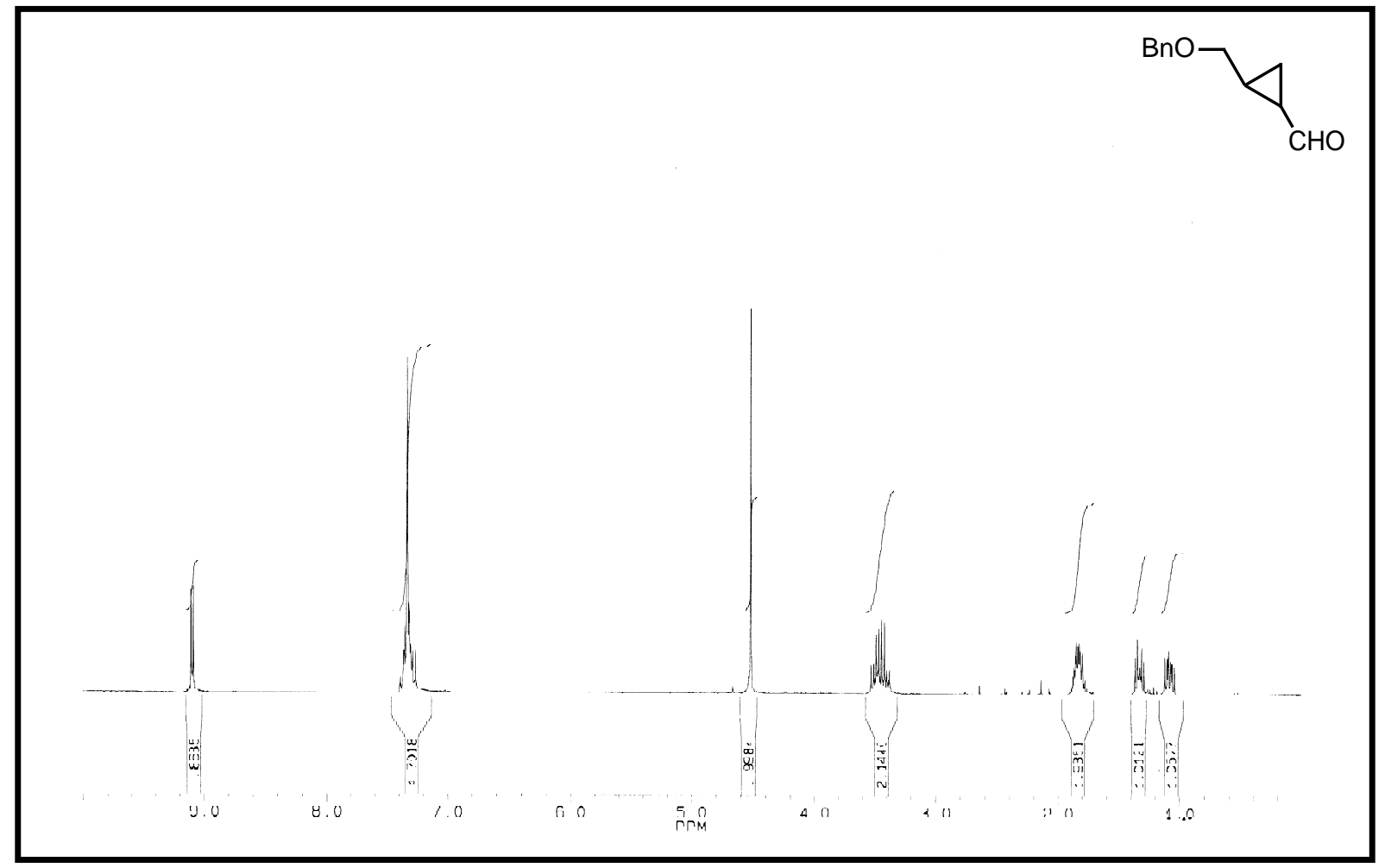

${ }^{1} H$-NMR-Spektrum von trans-2-Benzyloxymethylcyclopropan-1-carbaldehyd (48)

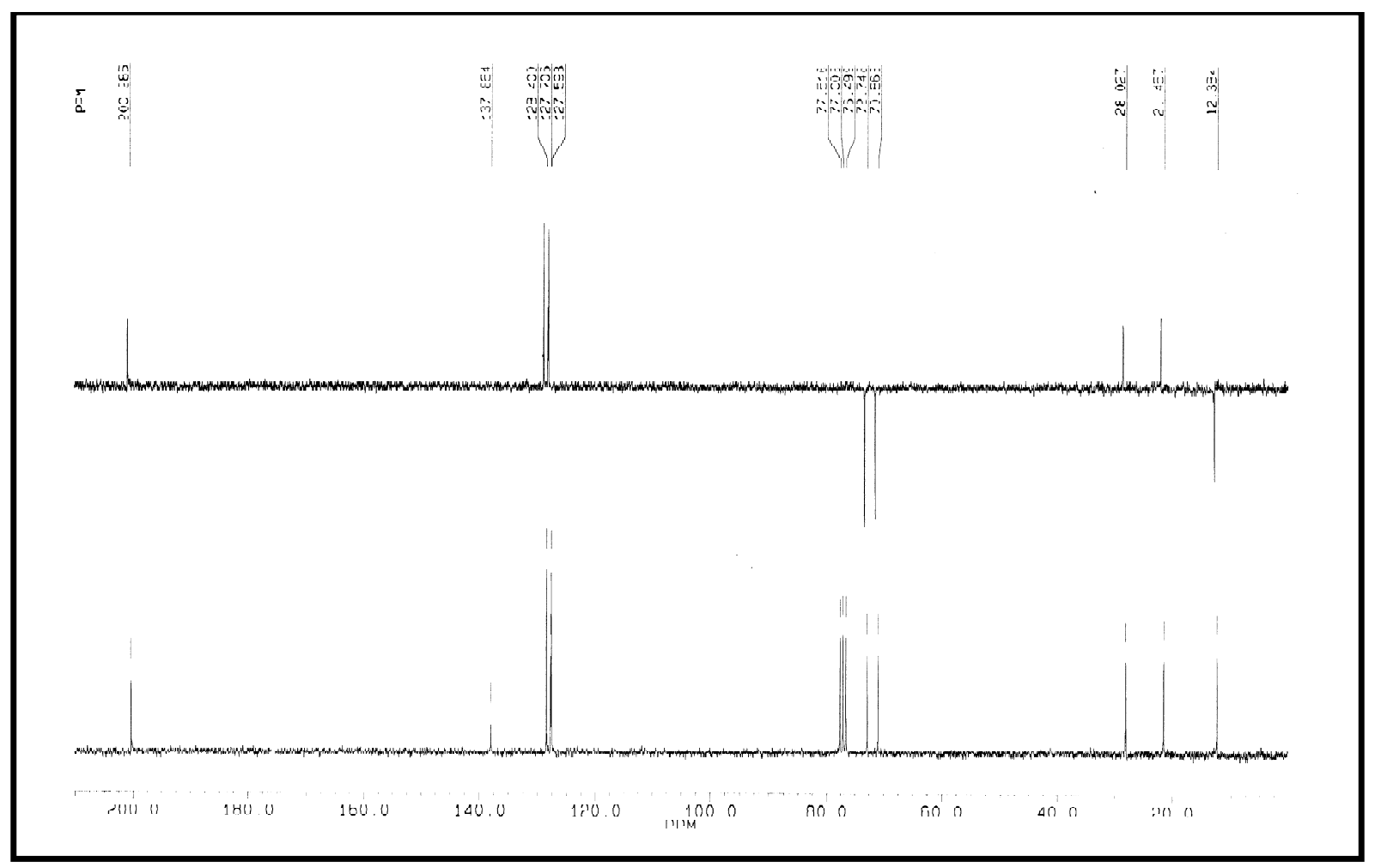

${ }^{13}$ C-NMR-Spektrum von trans-2-Benzyloxymethylcyclopropan-1-carbaldehyd (48) 


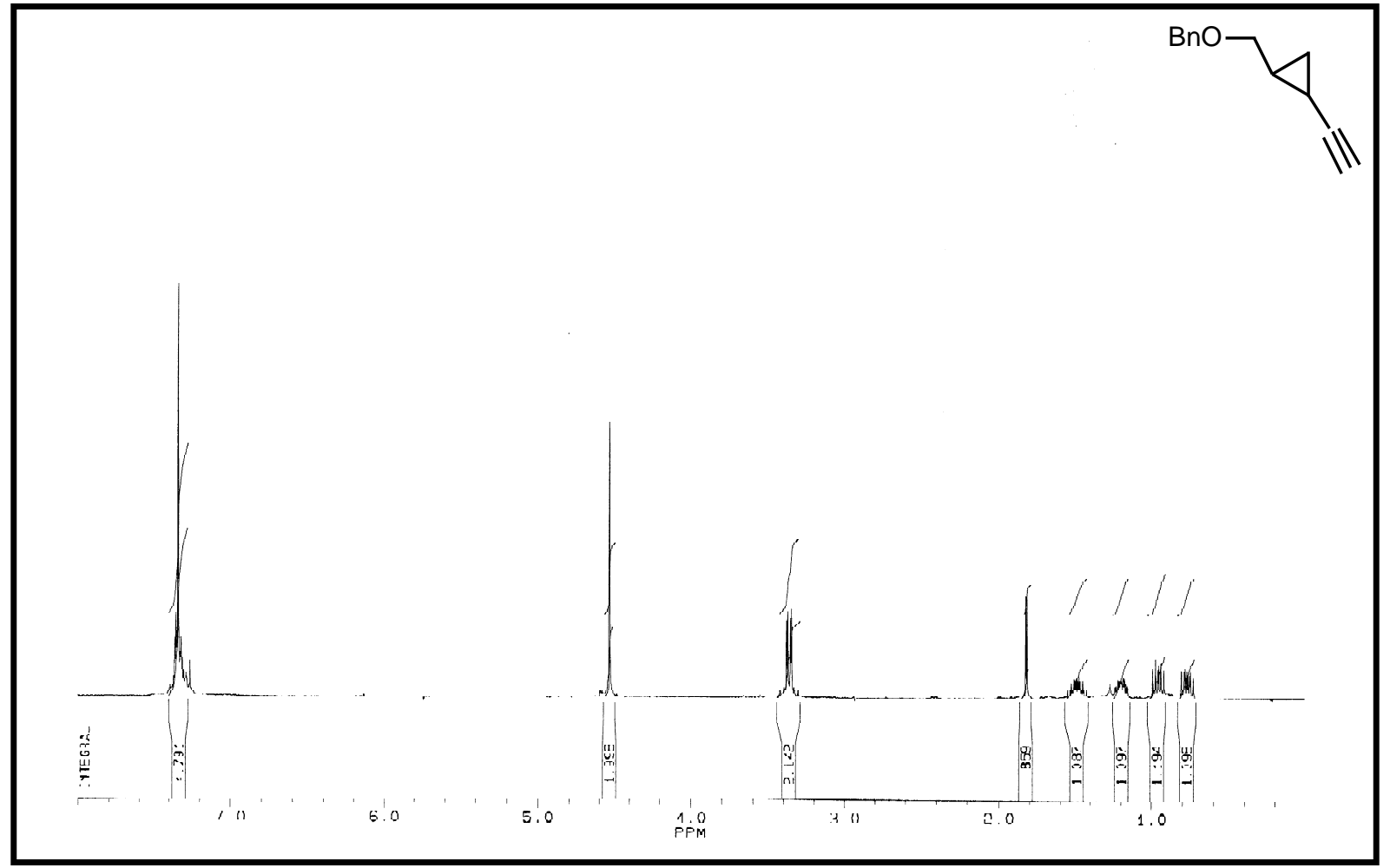

${ }^{1} H$-NMR-Spektrum von trans-2-Benzyloxymethyl-1-ethinylcyclopropan (53)

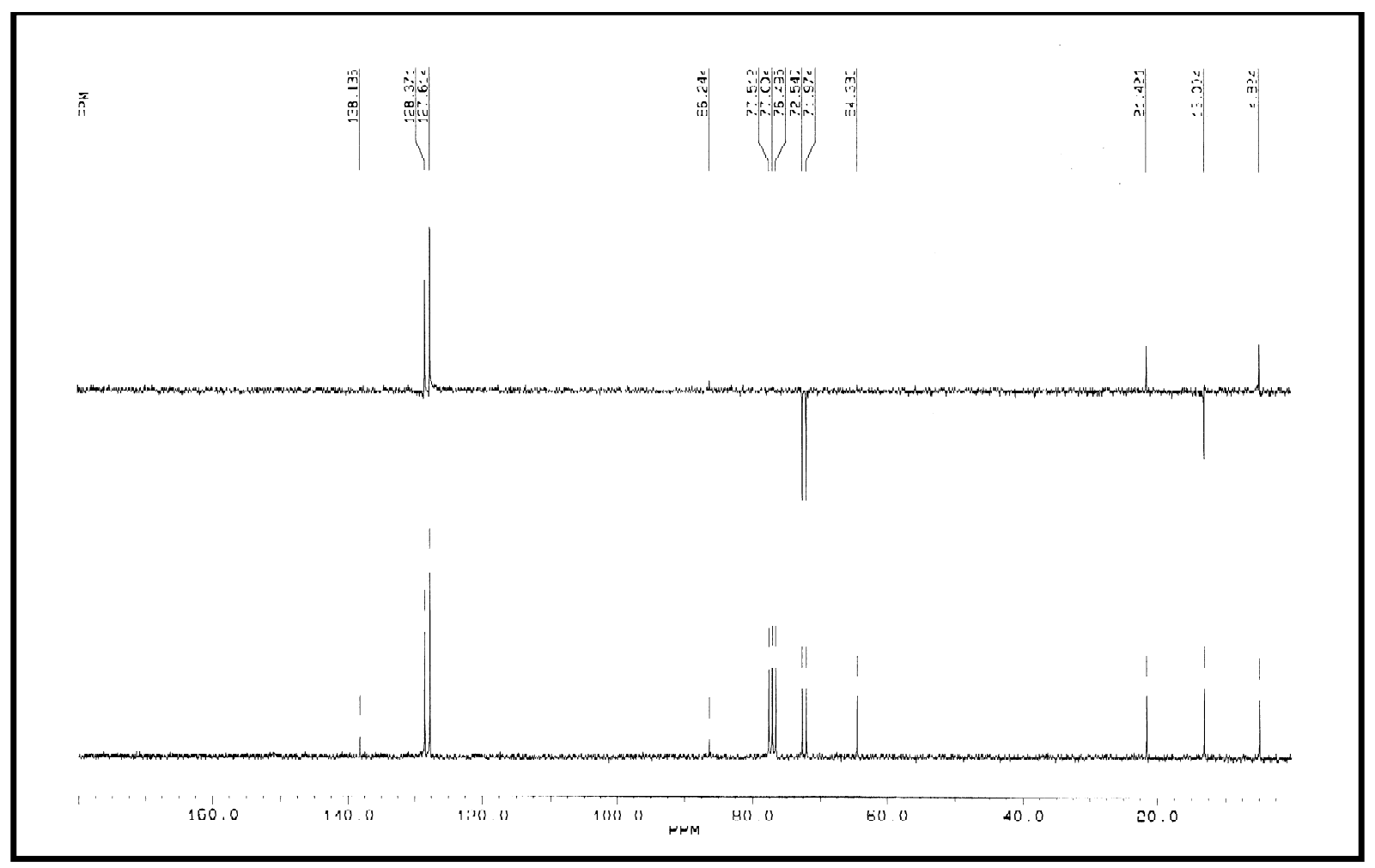

${ }^{13}$ C-NMR-Spektrum von trans-2-Benzyloxymethyl-1-ethinylcyclopropan (53) 


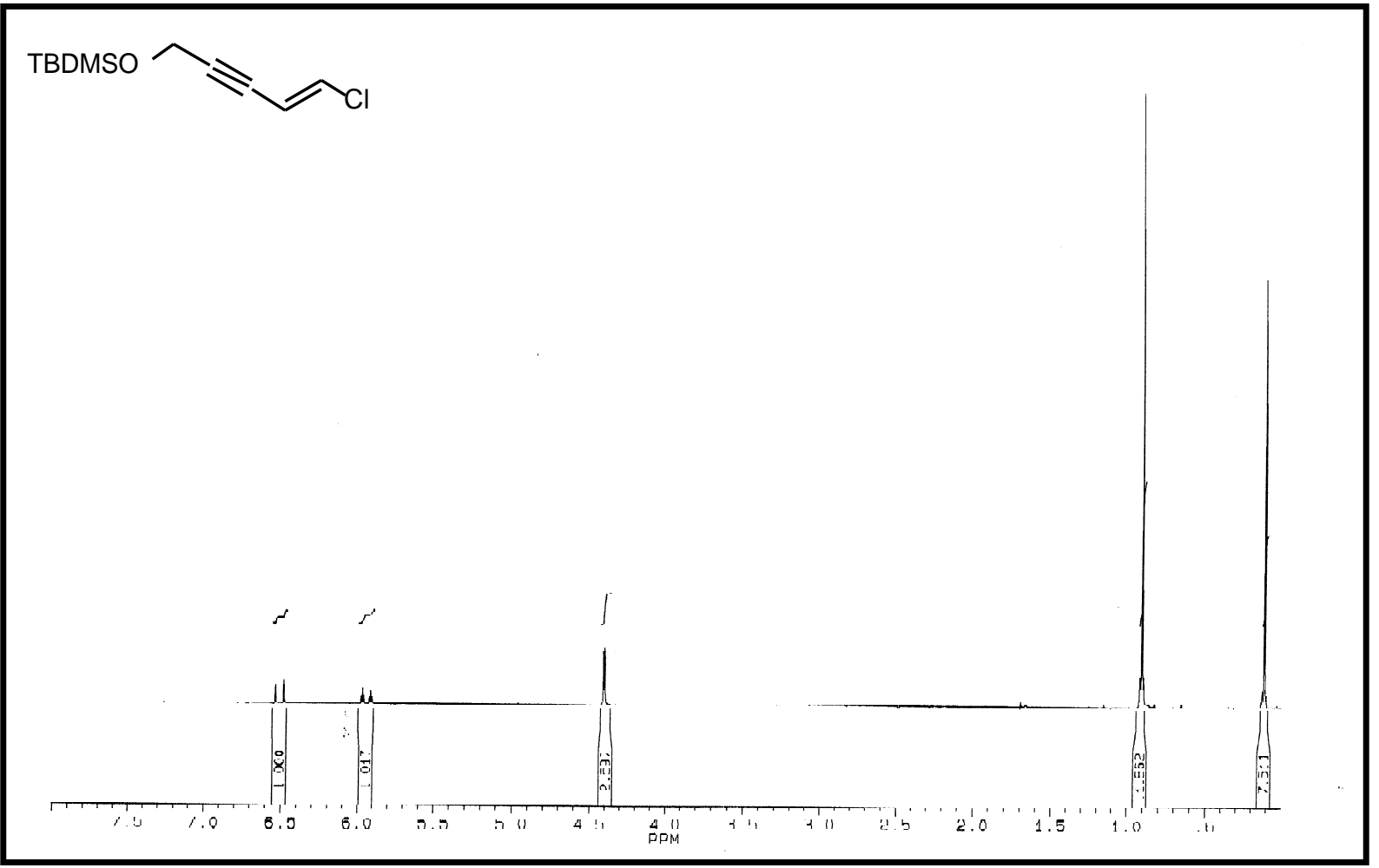

${ }^{1} H$-NMR-Spektrum von (E)-5-Chlor-1-(tert-butyl-dimethylsiloxy)-pent-2-in-4-en (93)

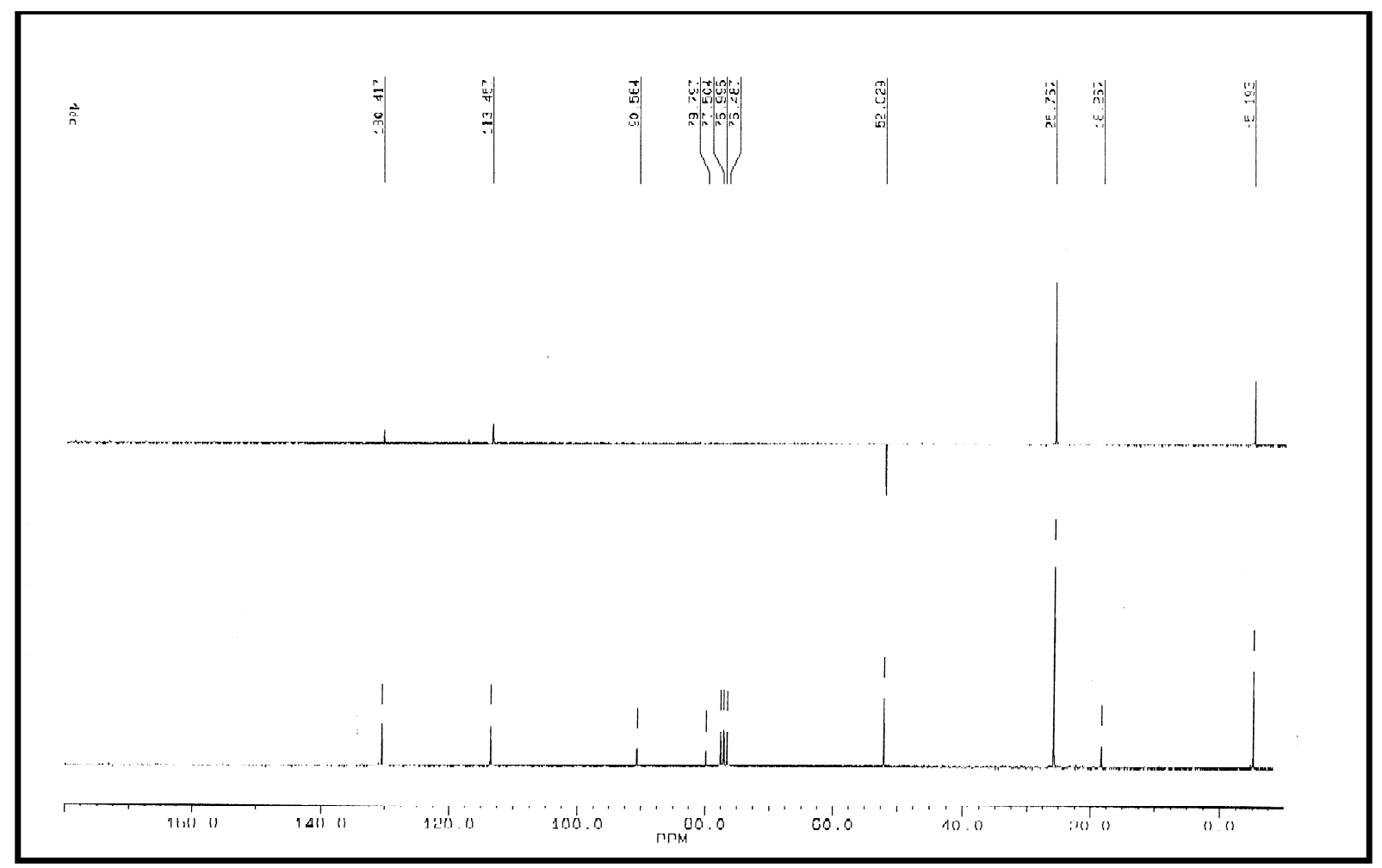

${ }^{13}$ C-NMR-Spektrum von (E)-5-Chlor-1-(tert-butyl-dimethylsiloxy)-pent-2-in-4-en (93) 


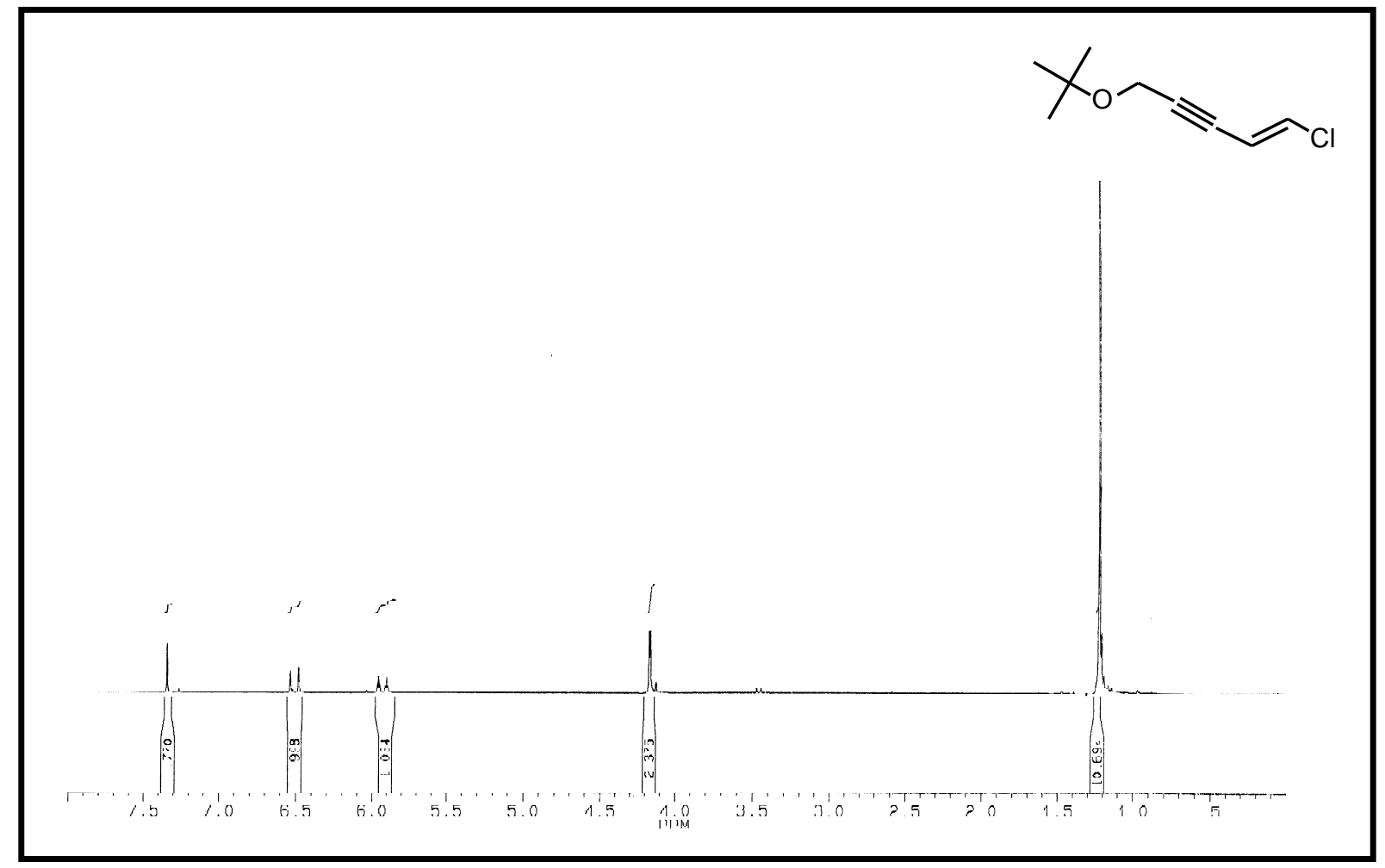

${ }^{1} H$-NMR-Spektrum von (E)-1-tert-Butyloxy-5-chlor-pent-2-in-4-en (92)

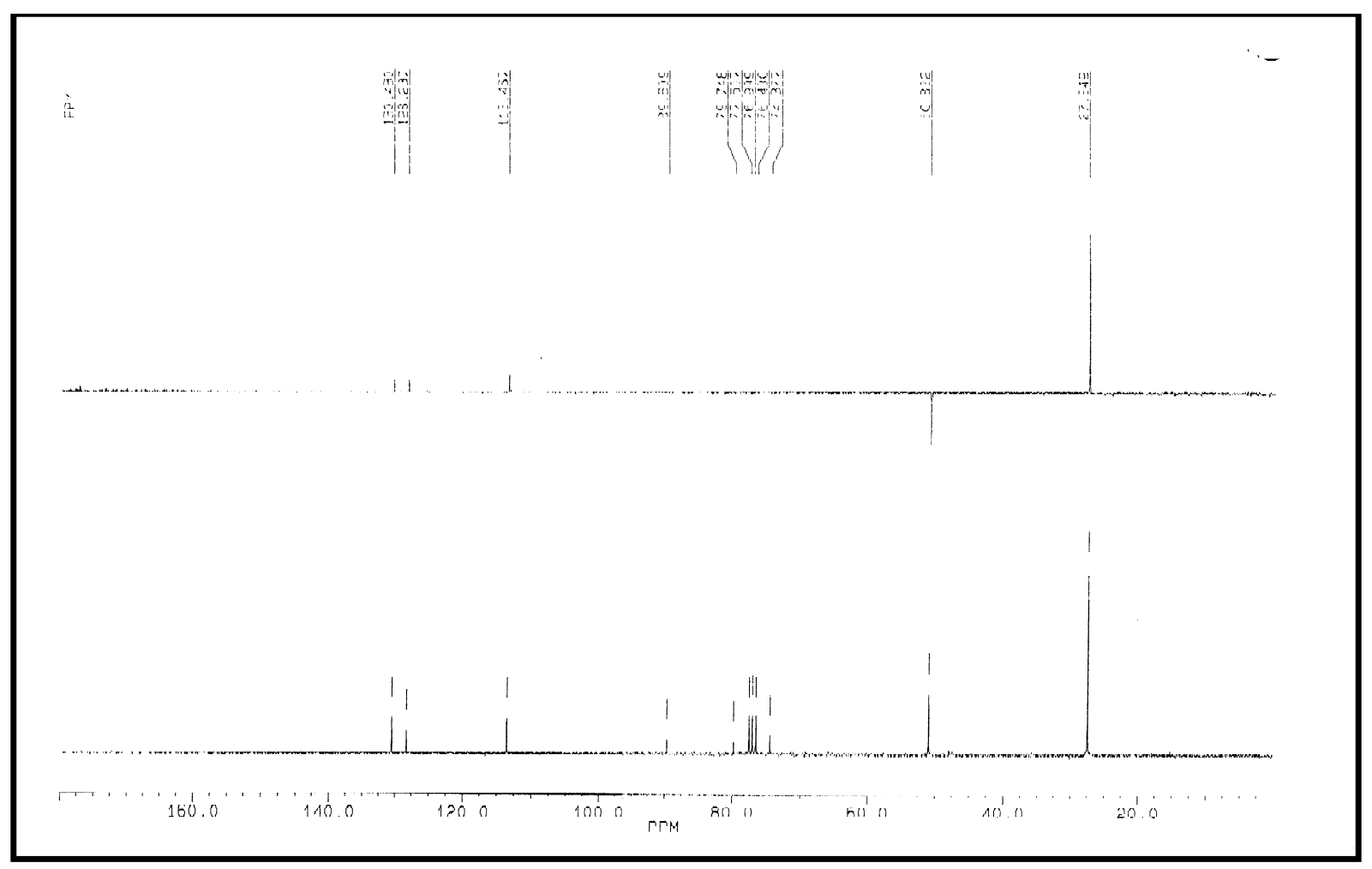

${ }^{13}$ C-NMR-Spektrum von (E)-1-tert-Butyloxy-5-chlor-pent-2-in-4-en (92) 


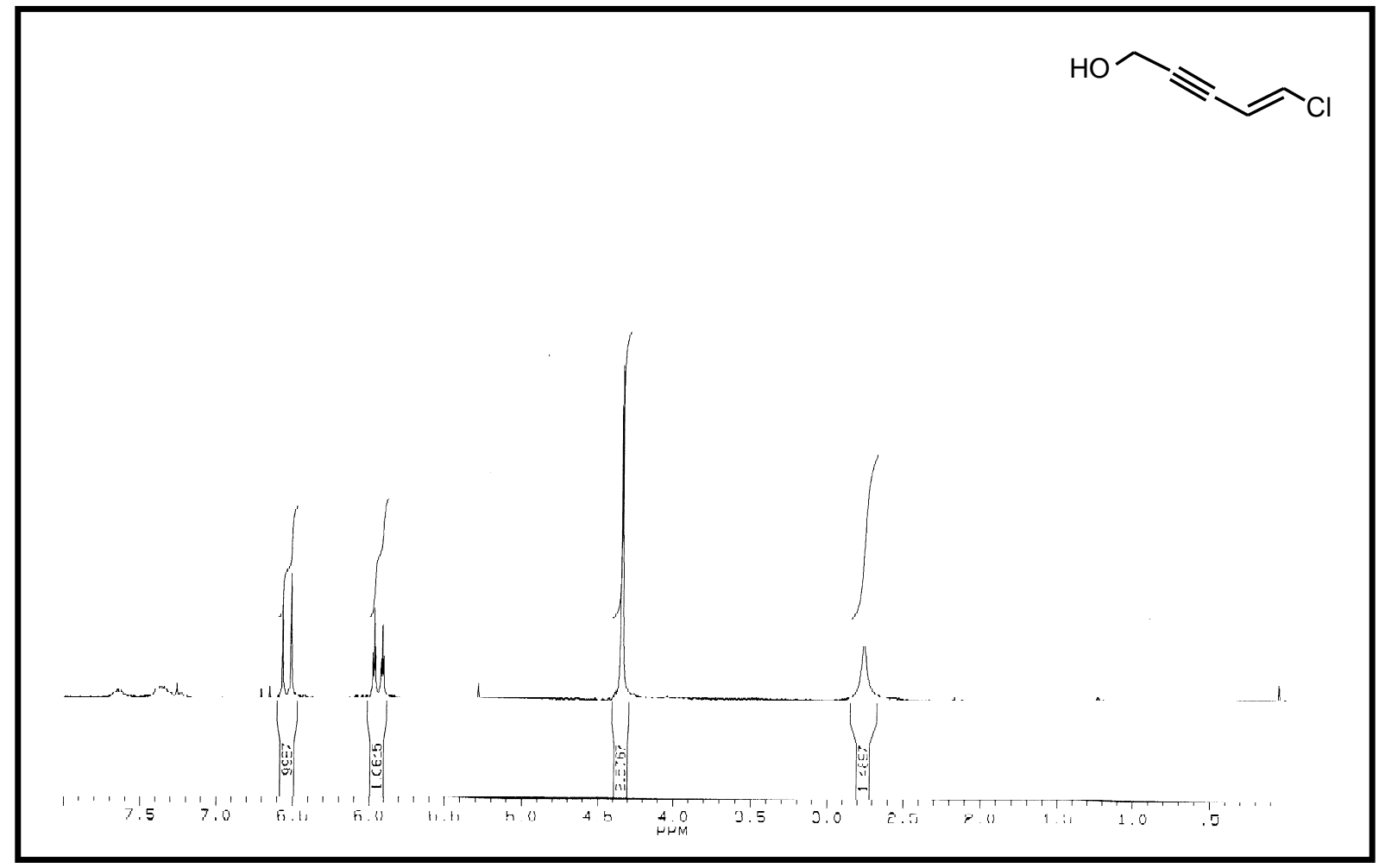

${ }^{1} H$-NMR-Spektrum von (E)-5-Chlor-pent-2-in-4-en-1-ol (94)

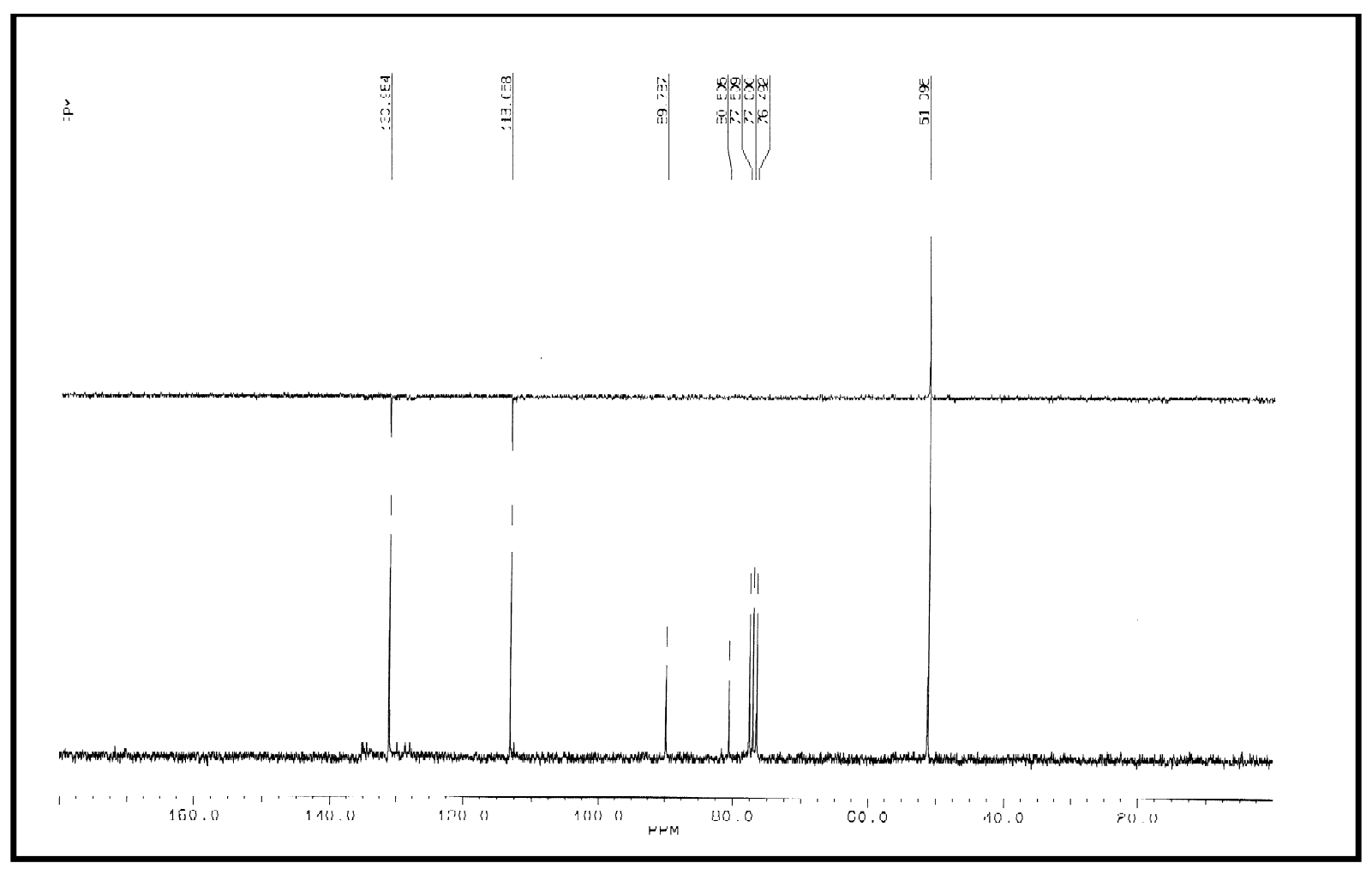

${ }^{13}$ C-NMR-Spektrum von (E)-5-Chlor-pent-2-in-4-en-1-ol (94) 


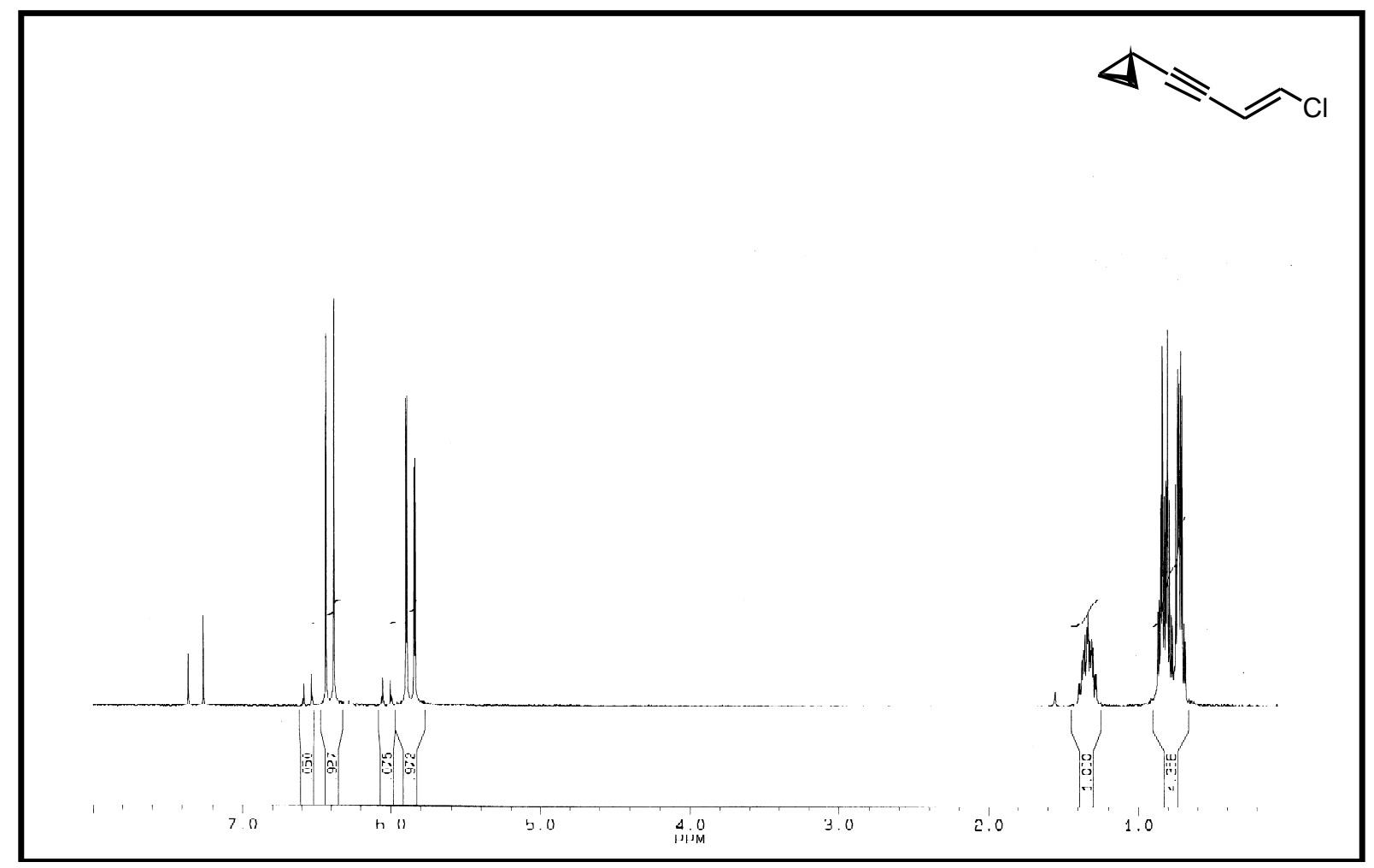

${ }^{1}$ H-NMR-Spektrum von (E)-4-Chlor-1-Cyclopropylbut-3-en-1-in (95)

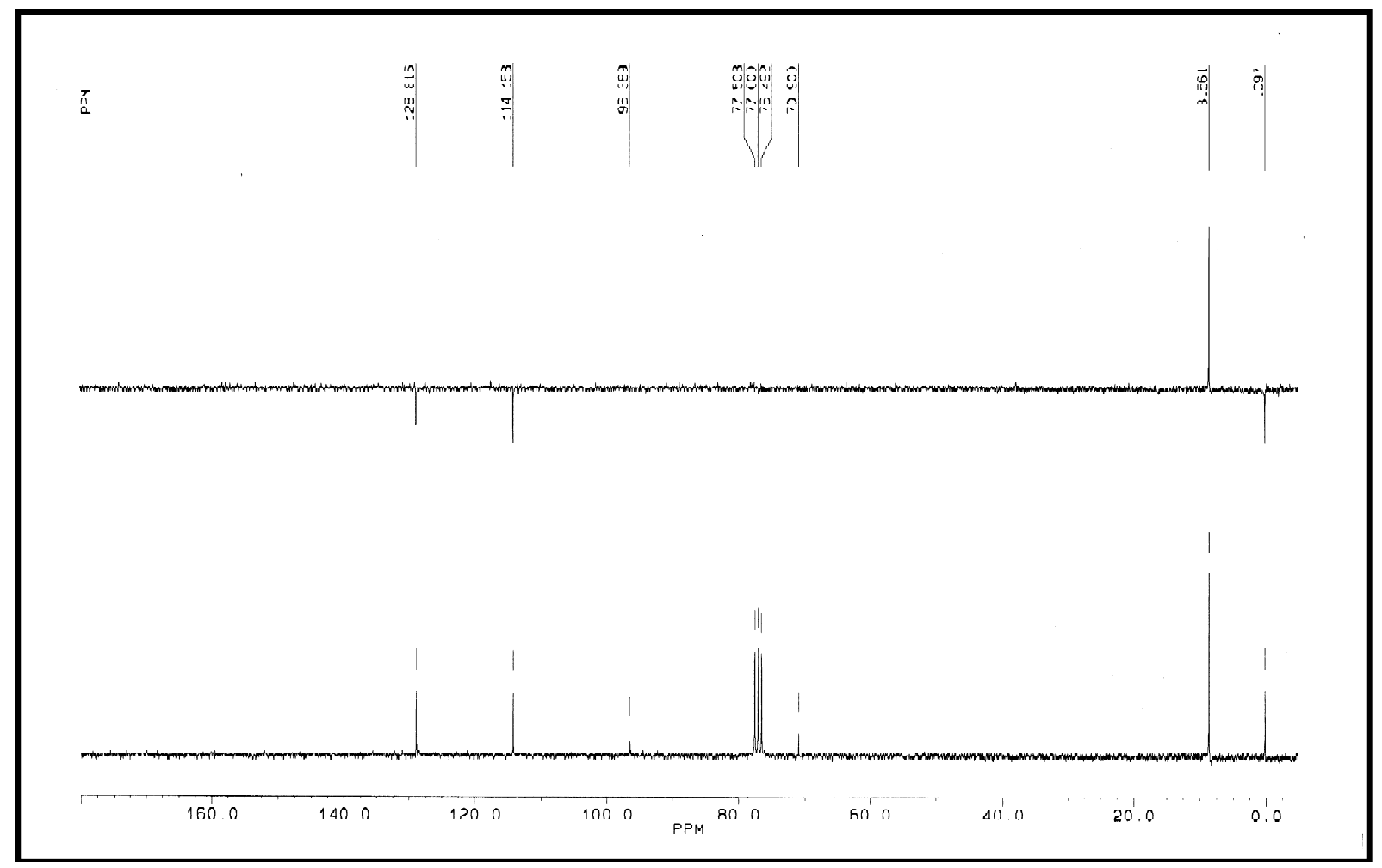

${ }^{13}$ C-NMR-Spektrum von (E)-4-Chlor-1-Cyclopropylbut-3-en-1-in (95) 


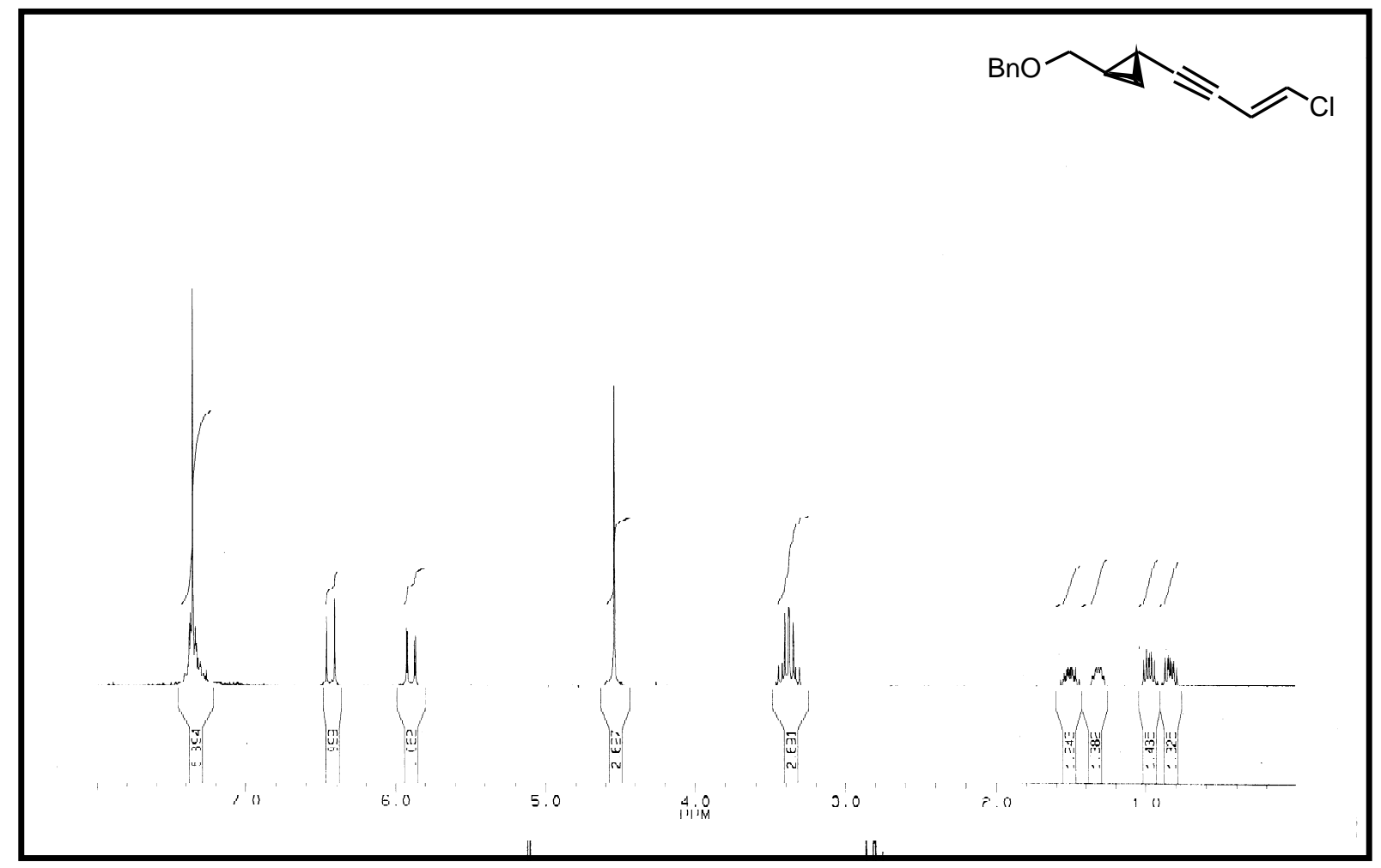

${ }^{1}$ H-NMR-Spektrum von (E)-4-Chlor-1-Benzyloxymethylcyclopropylbut-3-en-1-in (96)

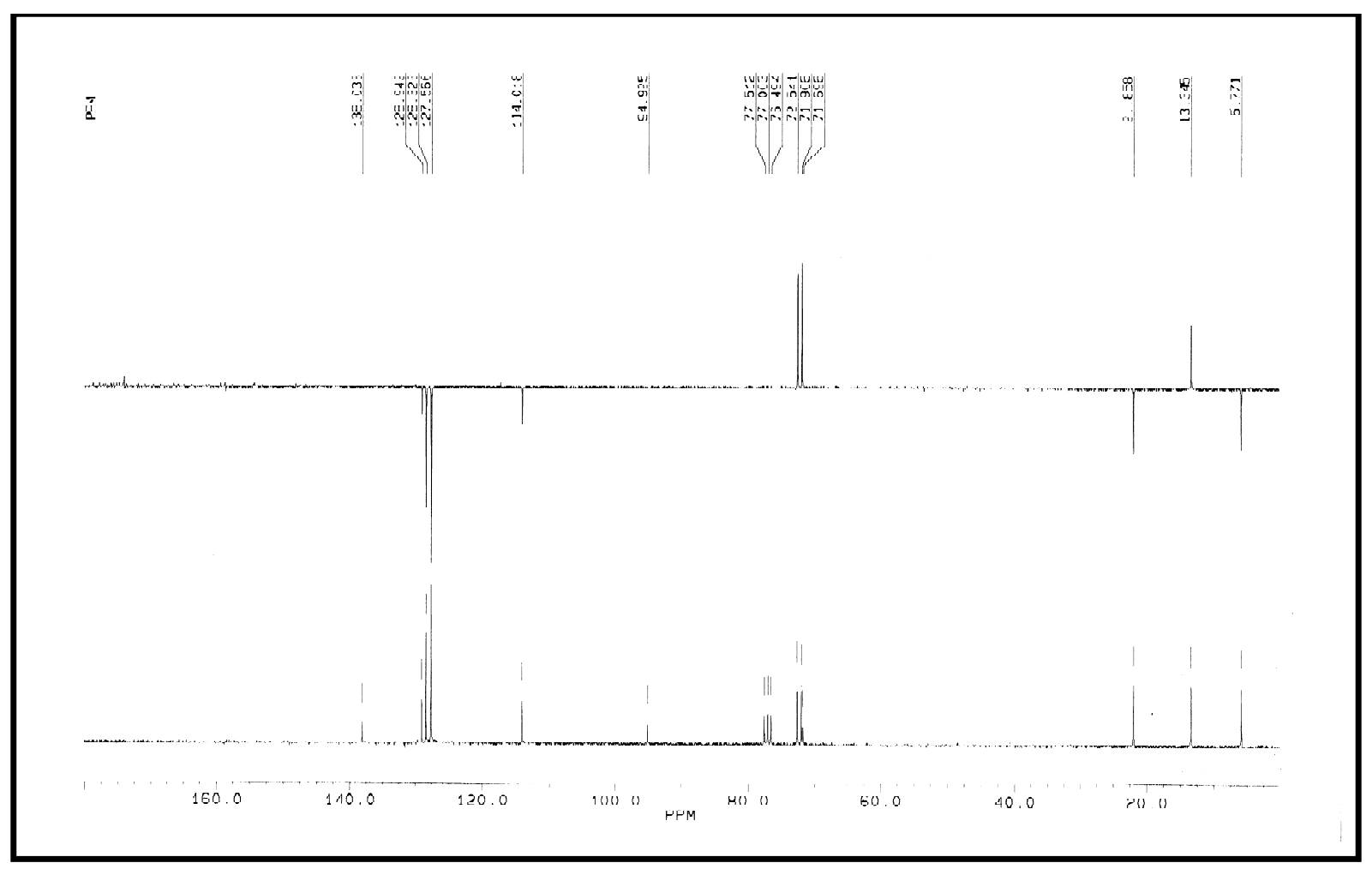

${ }^{13}$ C-NMR-Spektrum von (E)-4-Chlor-1-Benzyloxymethylcyclopropylbut-3-en-1-in (96) 


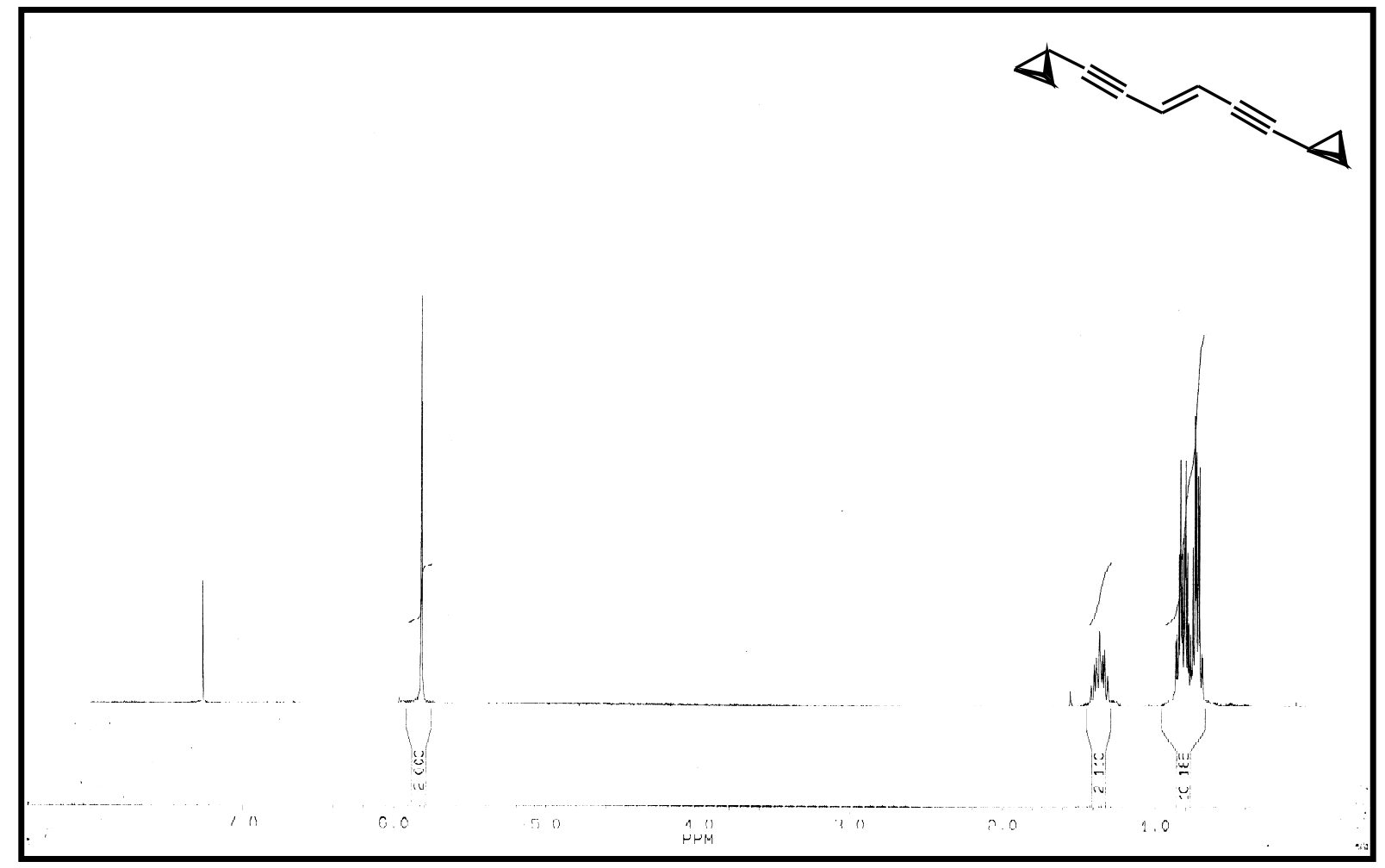

${ }^{1} H$-NMR-Spektrum von 1,6-Bis(cyclopropyl)hex-3-en-1,5-diin(98)

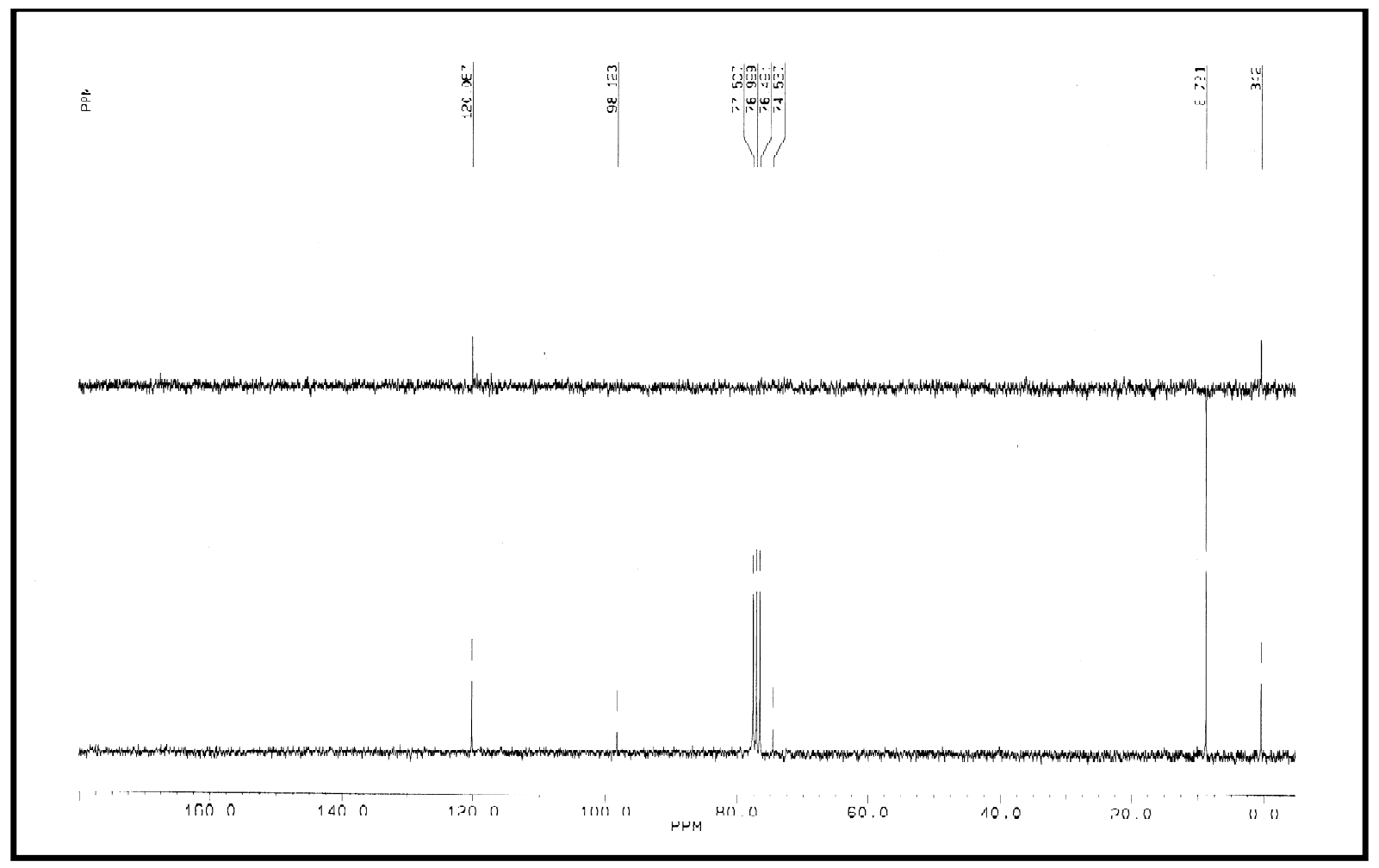

${ }^{13}$ C-NMR-Spektrum von 1,6-Bis(cyclopropyl)hex-3-en-1,5-diin (98) 


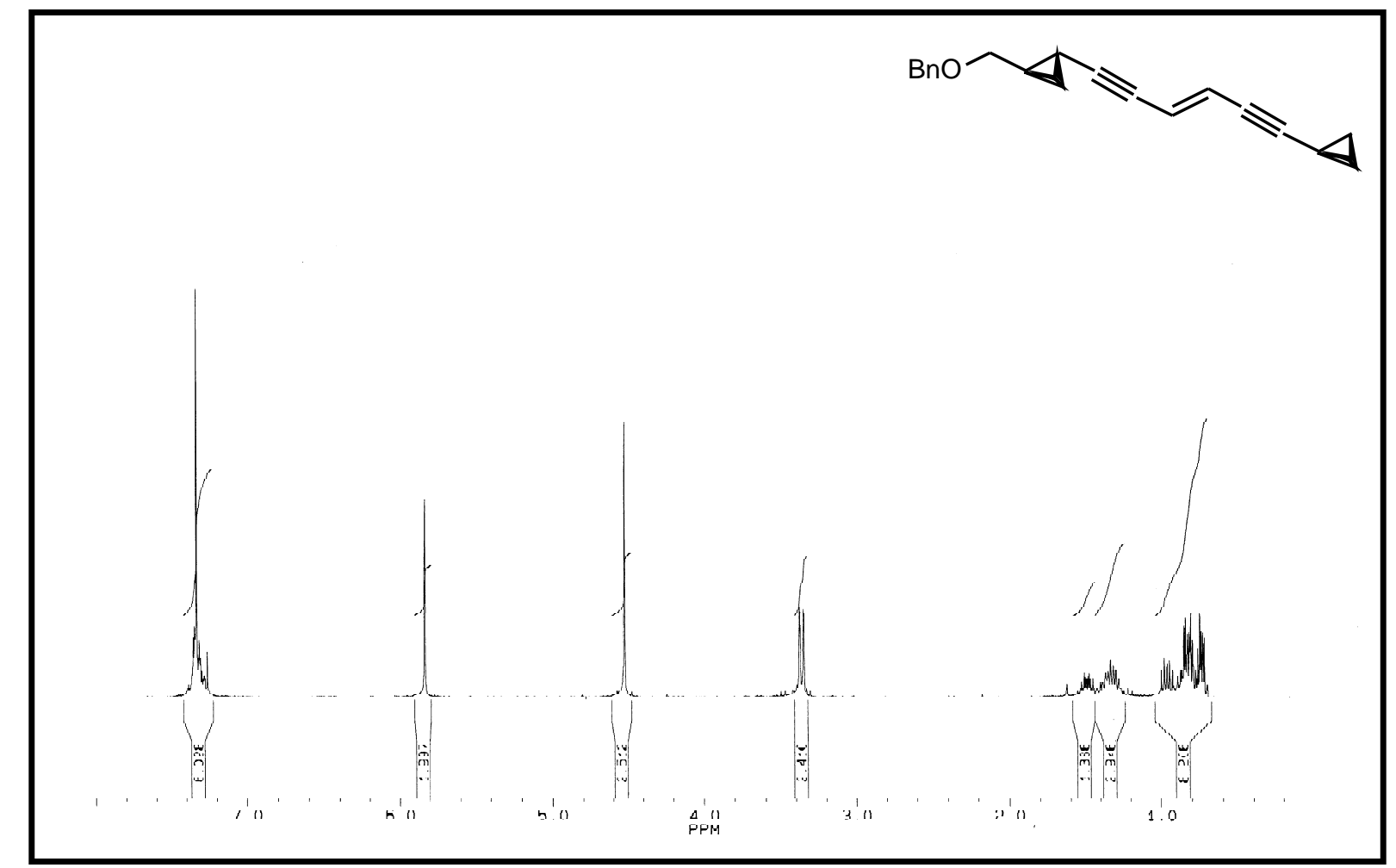

${ }^{1}$ H-NMR-Spektrum von 1-(Benzyloxymethylcyclopropyl)-6-cyclopropylhex-3-en-1,5-diin (99)

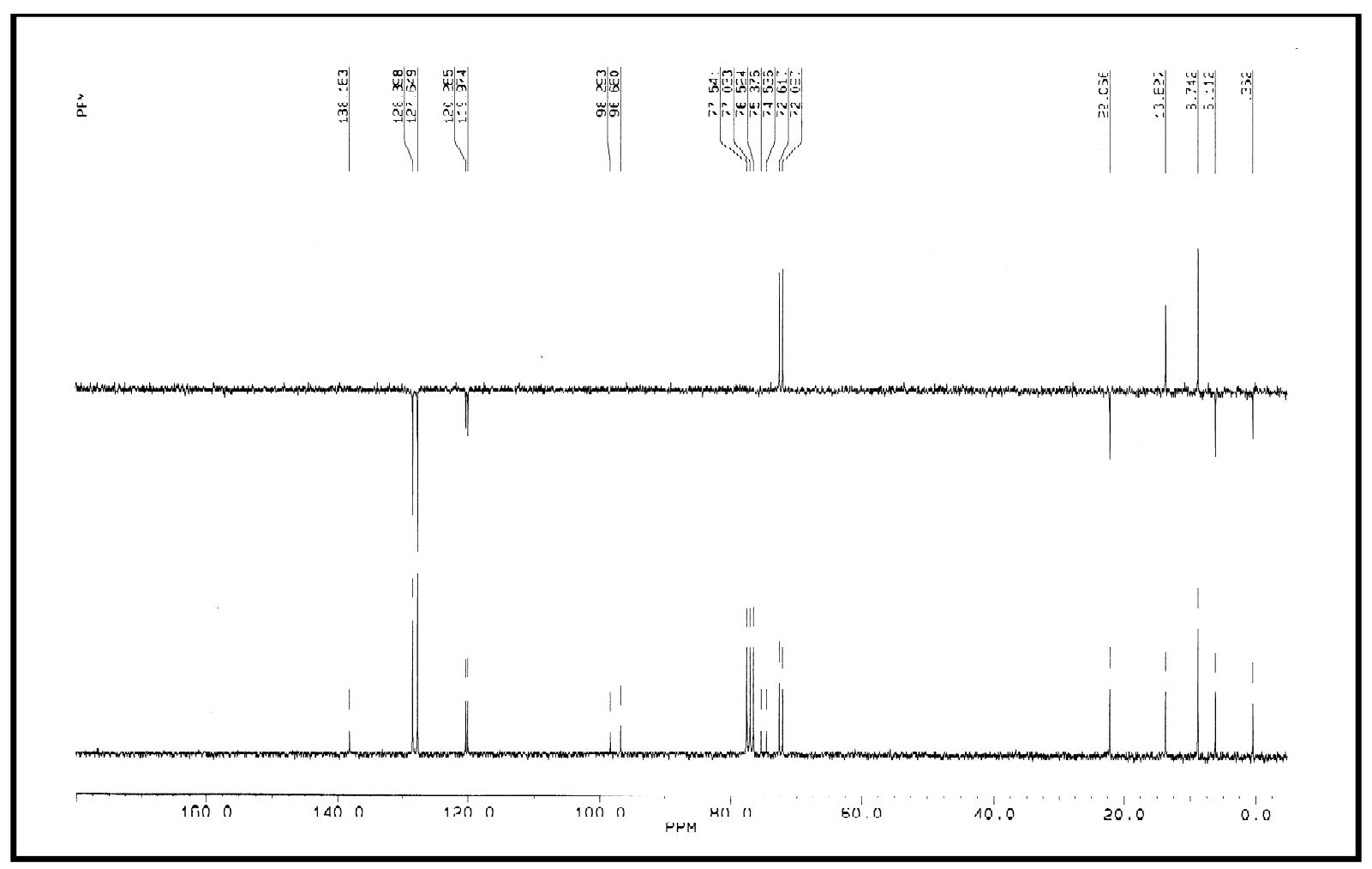

${ }^{13}$ C-NMR-Spektrum von 1-(Benzyloxymethylcyclopropyl)-6-cyclopropylhex-3-en-1,5-diin (99) 

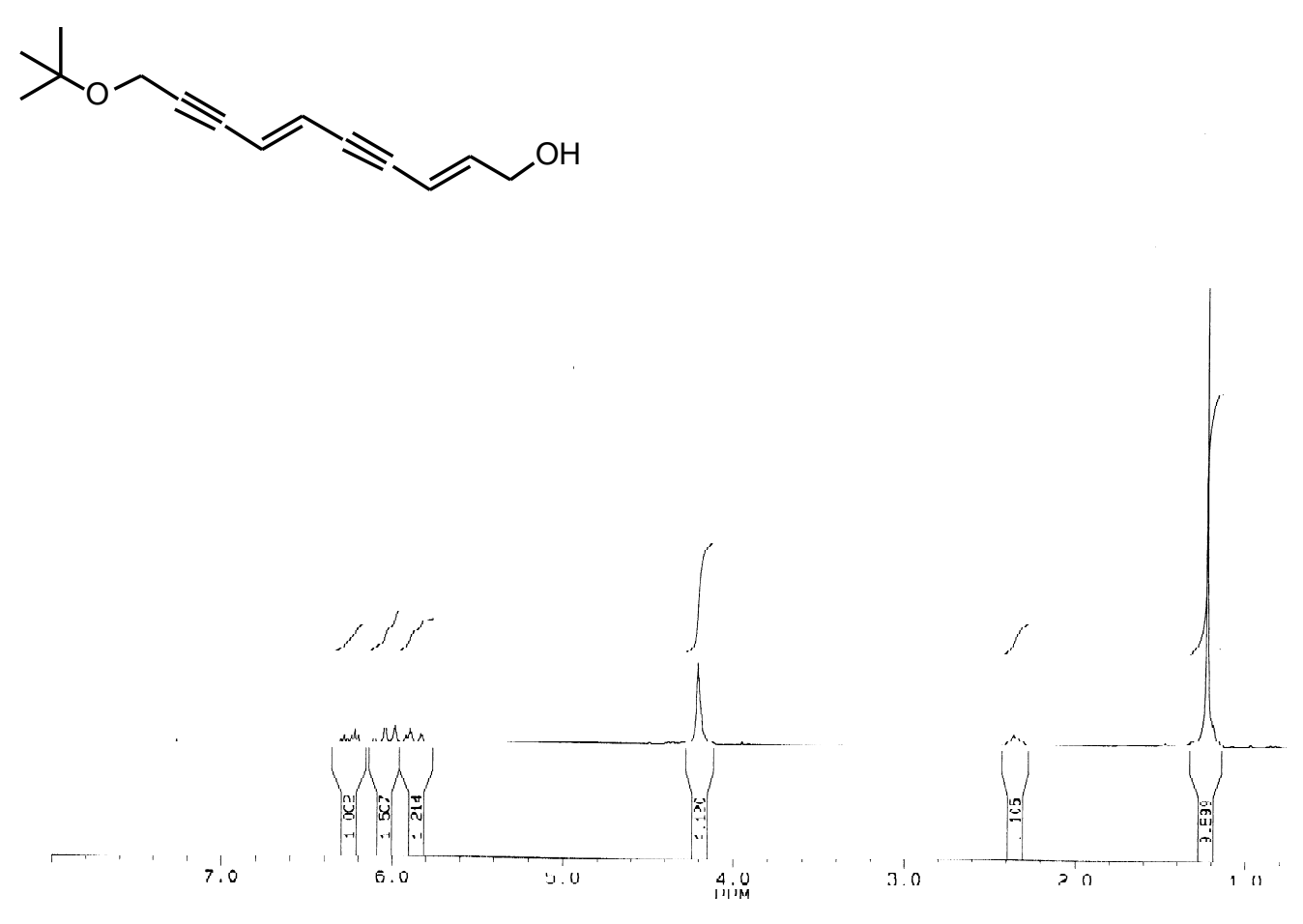

${ }^{1} H$-NMR-Spektrum von (E,E)-1-tert-Butoxy-dec-2,6-diin-4,8-dien-10-ol (100)

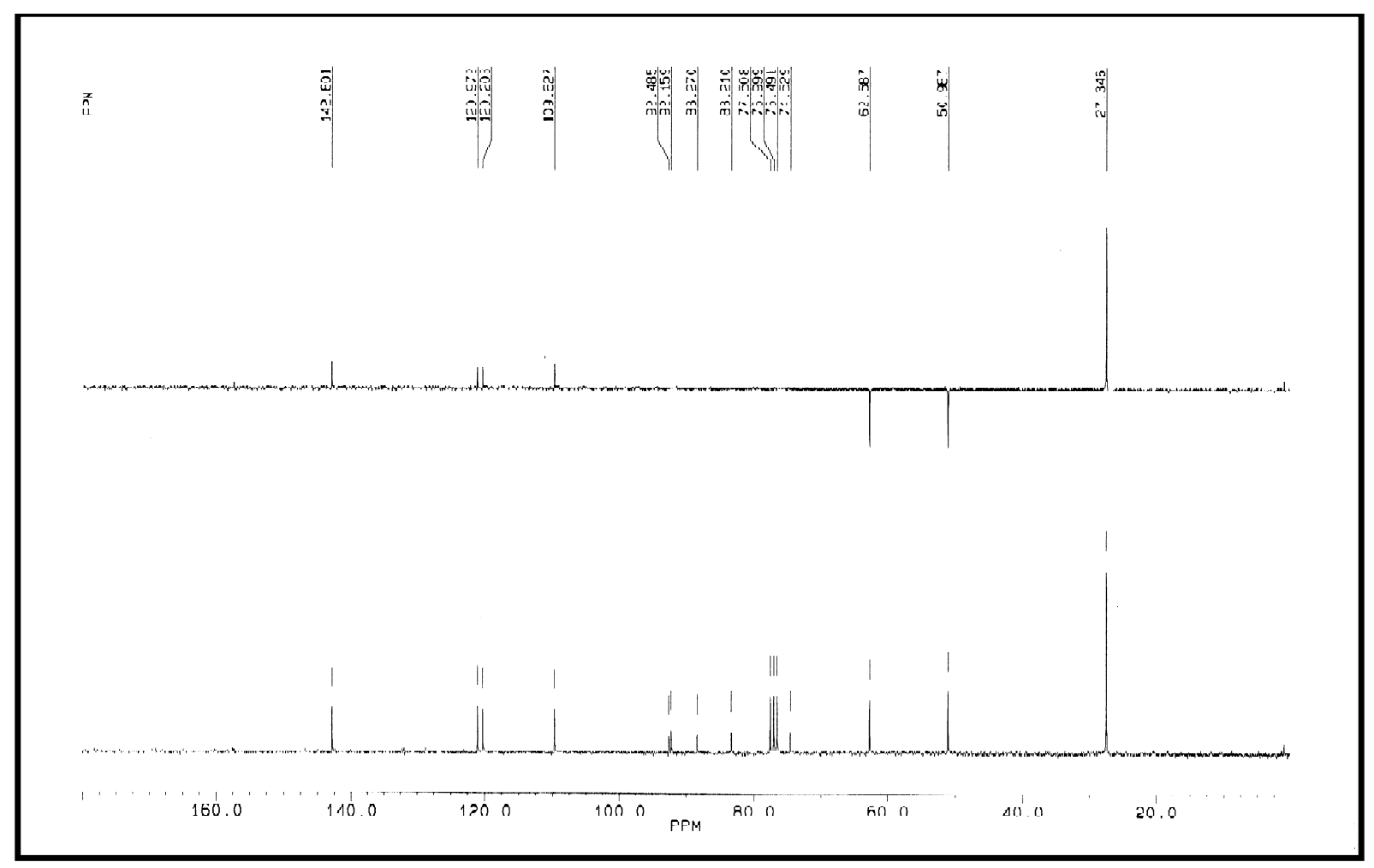

${ }^{13}$ C-NMR-Spektrum von (E,E)-1-tert-Butoxy-dec-2,6-diin-4,8-dien-10-ol (100) 


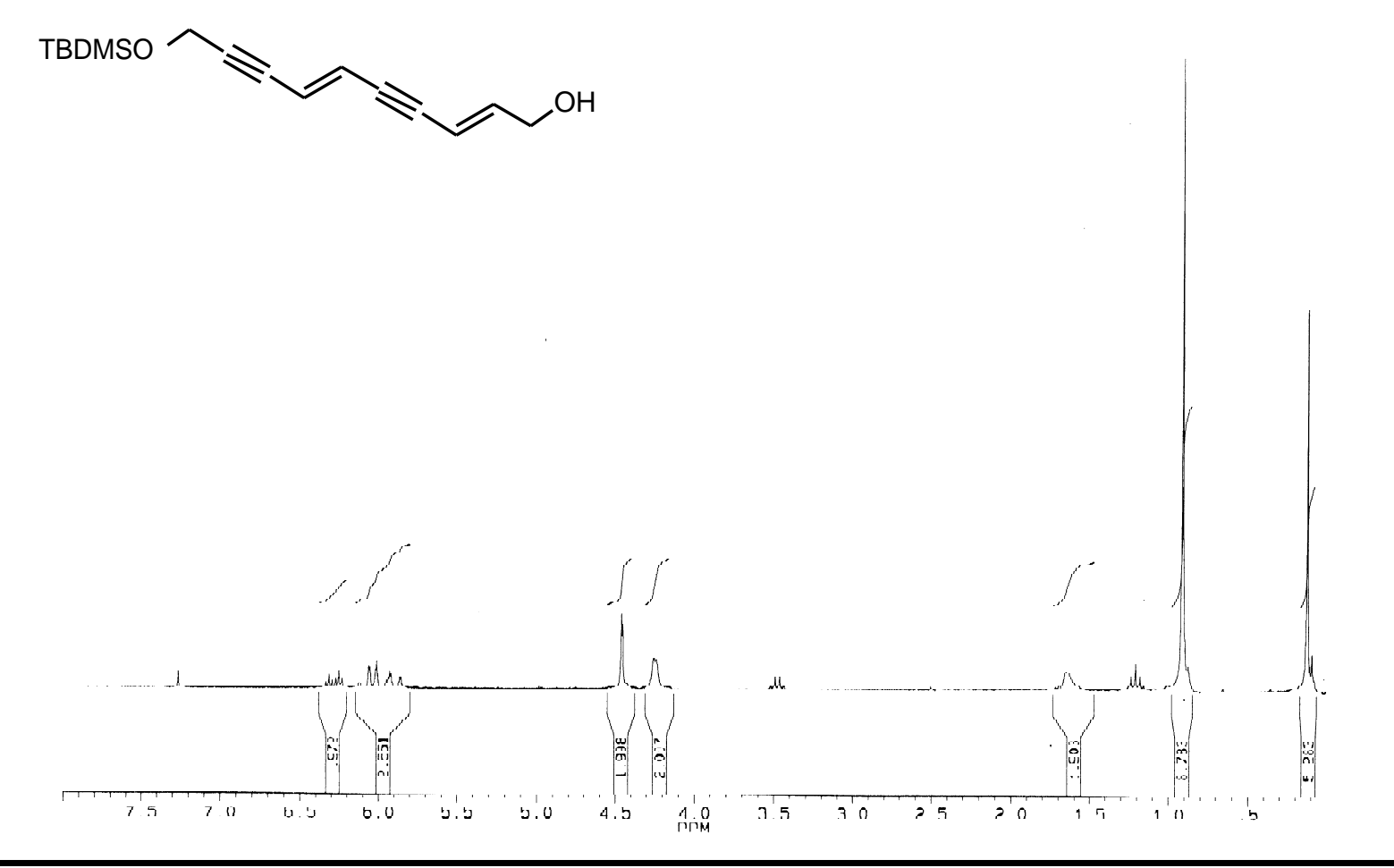

${ }^{1} H$-NMR-Spektrum von (E,E)-1-tert-Butyldimethylsiloxy-dec-2,6-diin-4,8-dien-10-ol (101)

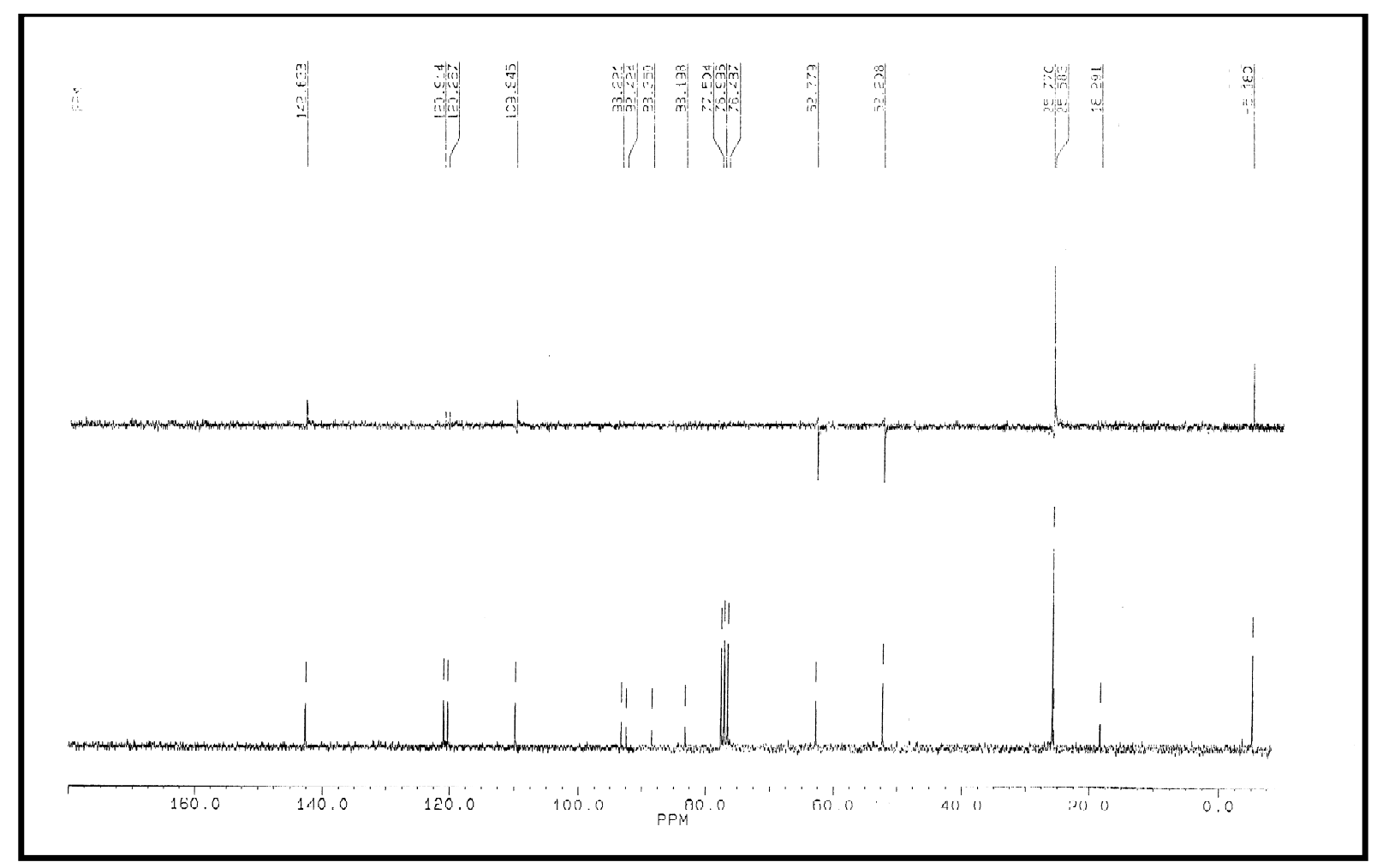

${ }^{13}$ C-NMR-Spektrum von (E,E)-1-tert-Butyldimethylsiloxy-dec-2,6-diin-4,8-dien-10-ol (101) 

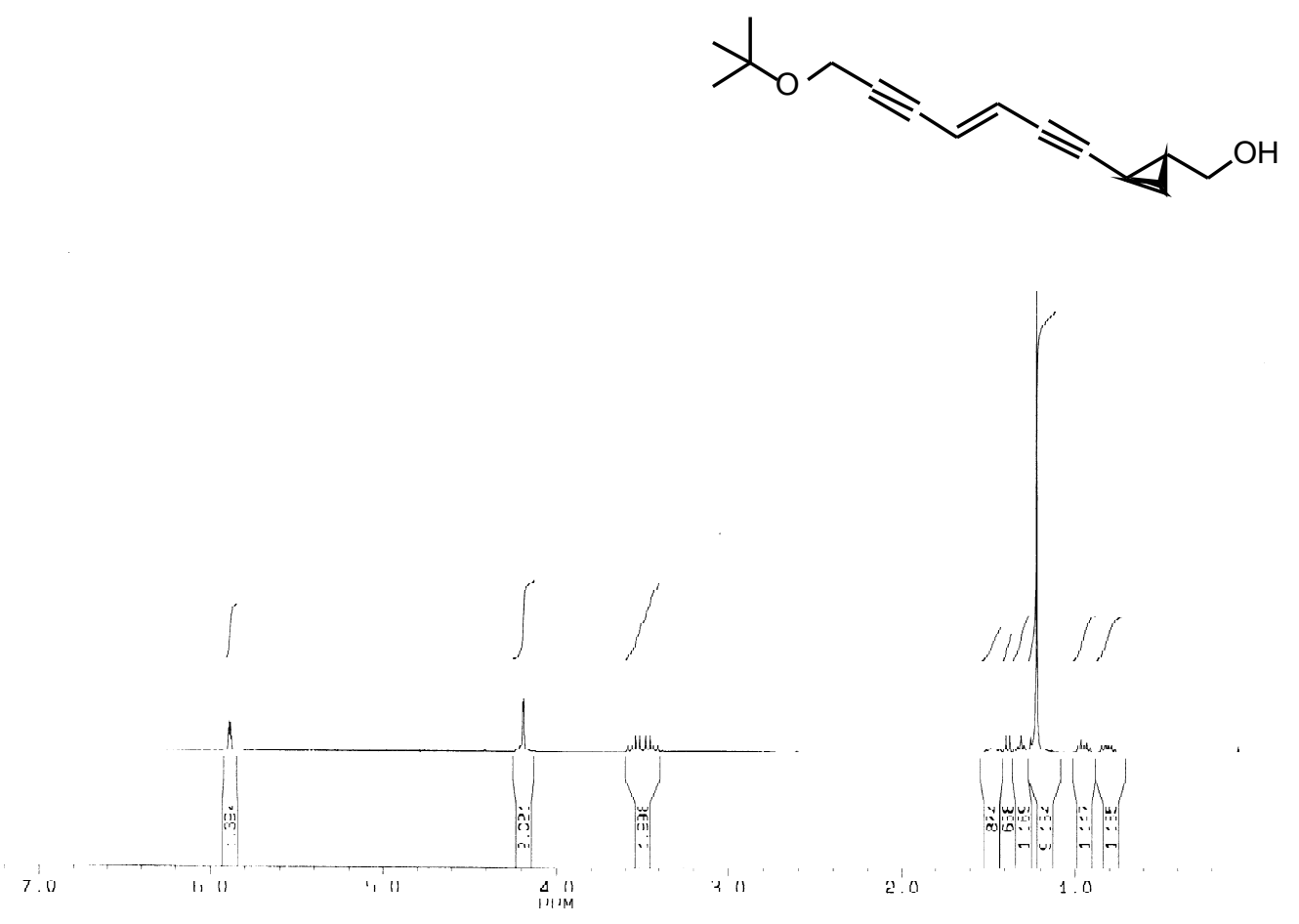

${ }^{1}$ H-NMR-Spektrum von (E)-1-tert-Butoxy-7-hydroxymethylcyclopropyl-hept-2,6-in-4-en (103)

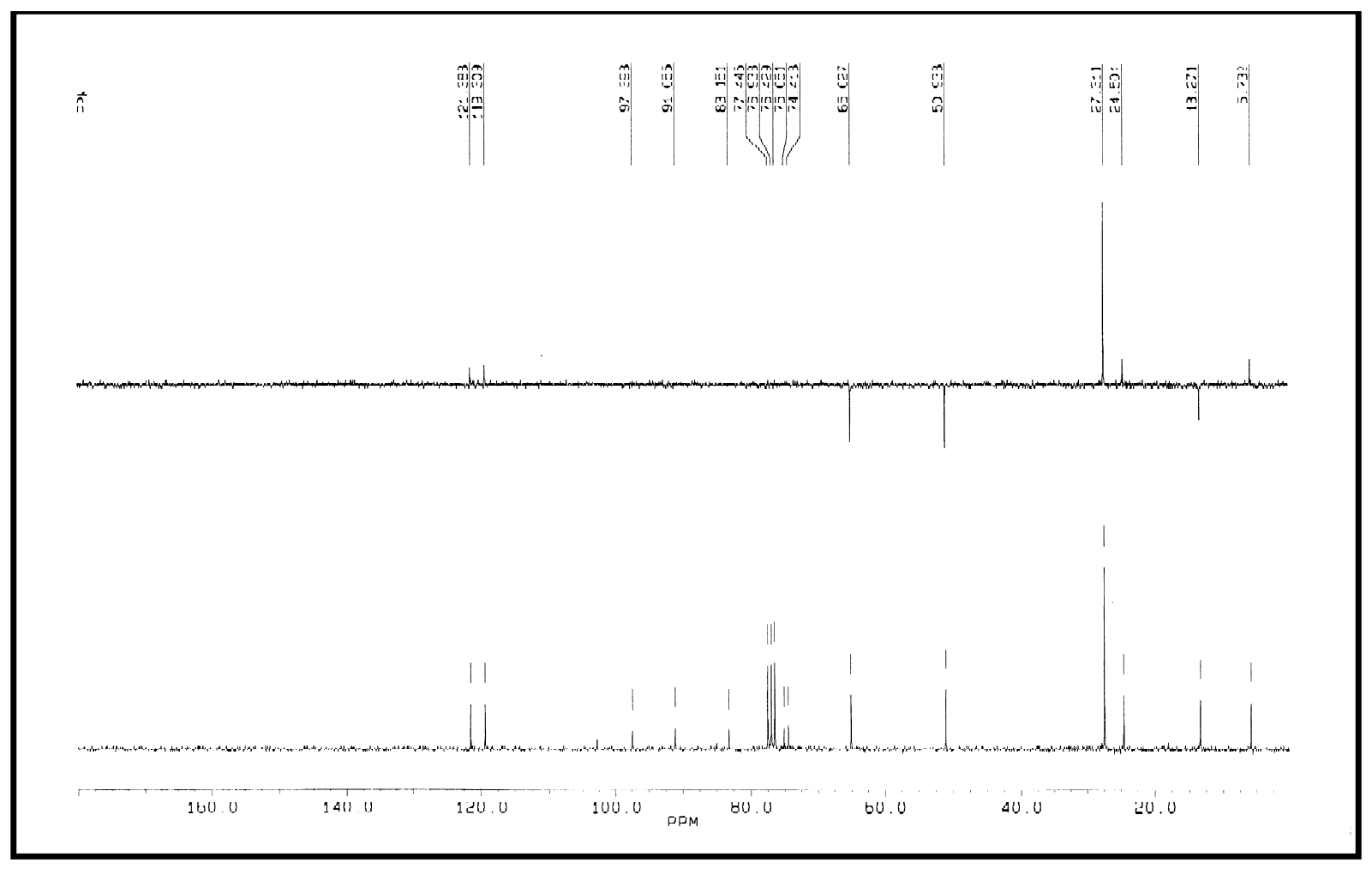

${ }^{13}$ C-NMR-Spektrum von (E)-1-tert-Butoxy-7-hydroxymethylcyclopropyl-hept-2,6-in-4-en (103) 


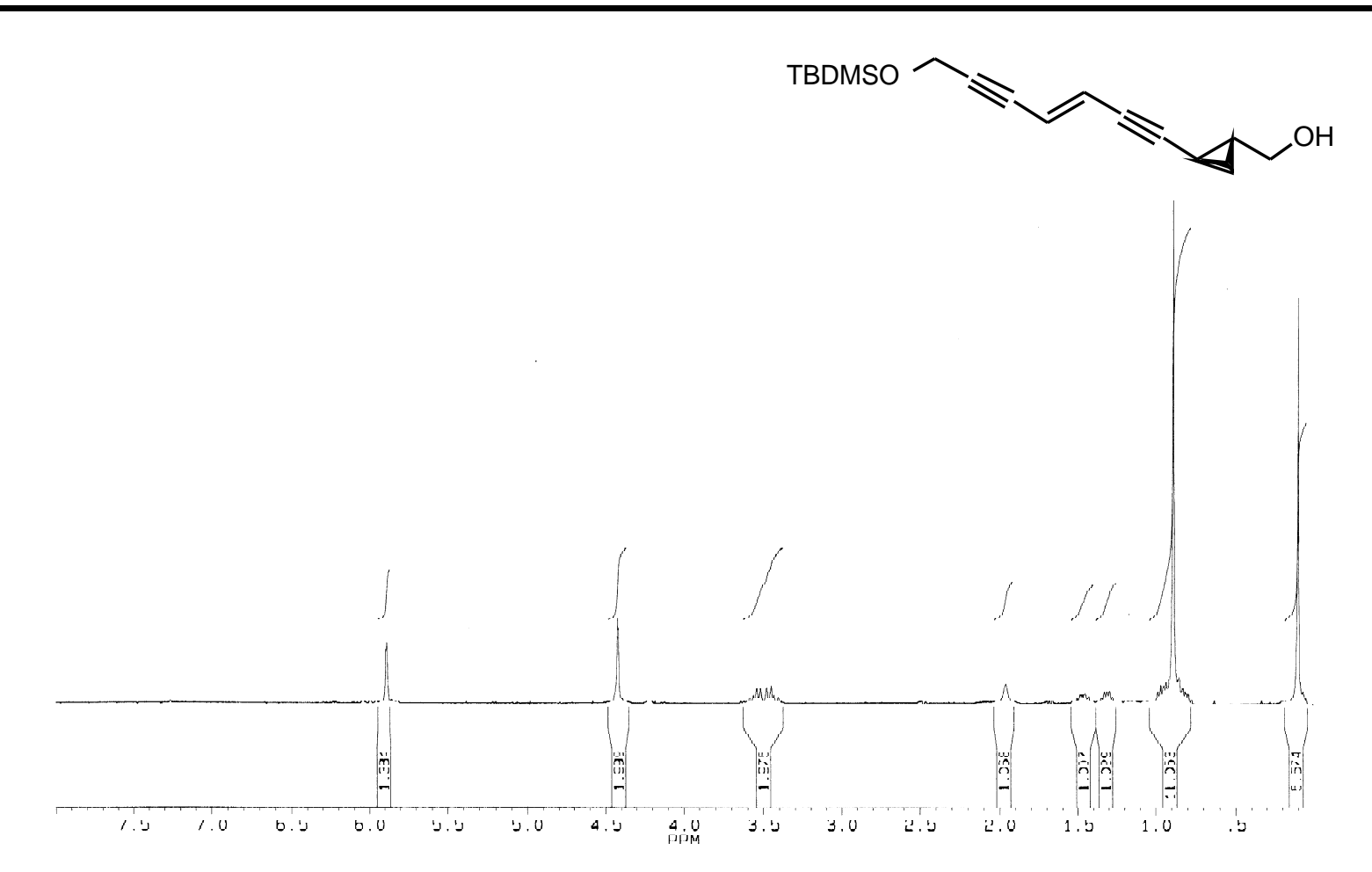

${ }^{1} H$-NMR-Spektrum von (E)-1-tert-Butyldimethylsiloxy-7-hydroxymethylcyclopropyl-hept-2,6in-4-en (104)

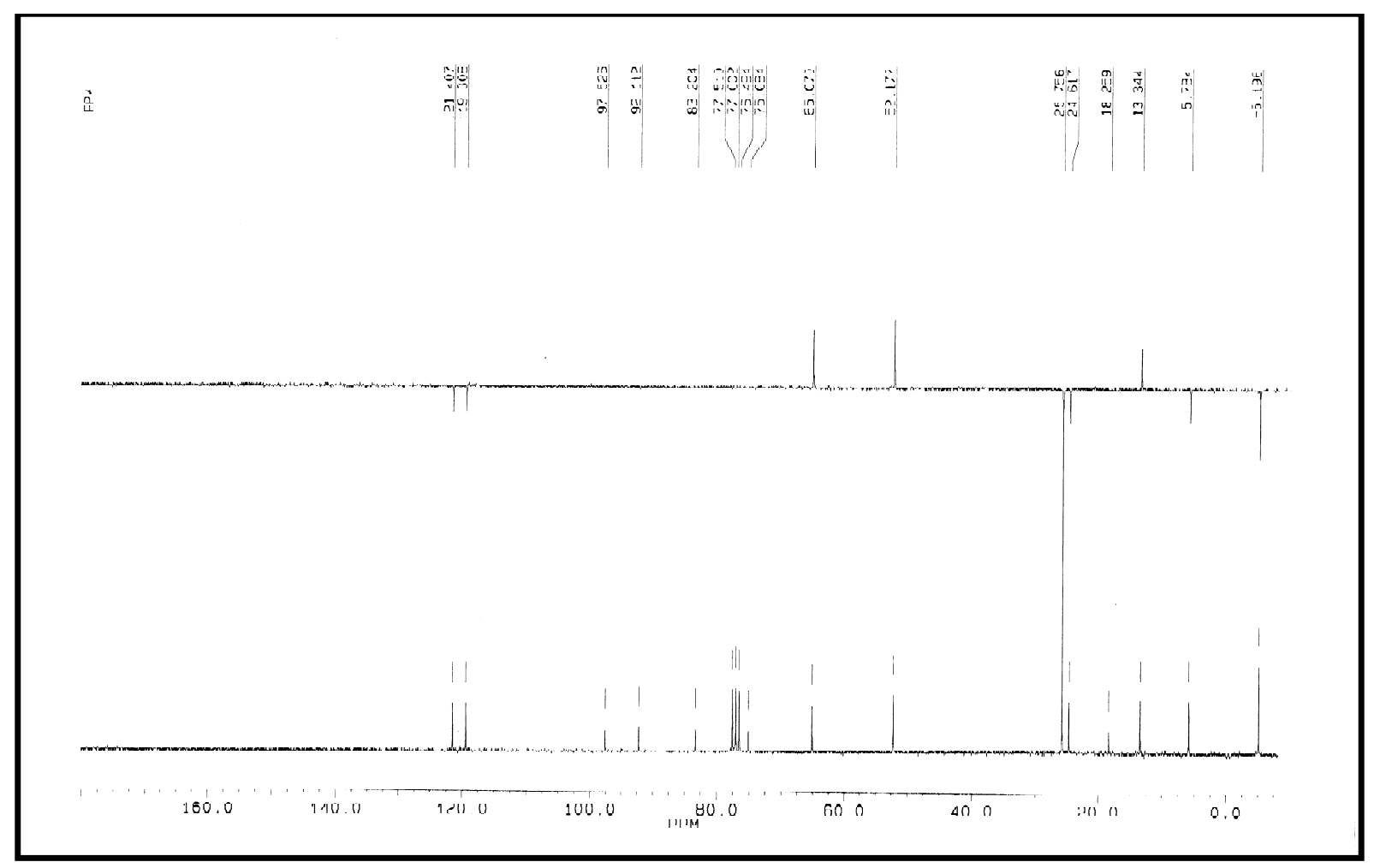

${ }^{13}$ C-NMR-Spektrum von (E)-1-tert-Butyldimethylsiloxy-7-hydroxymethylcyclopropyl-hept-2,6in-4-en (105) 

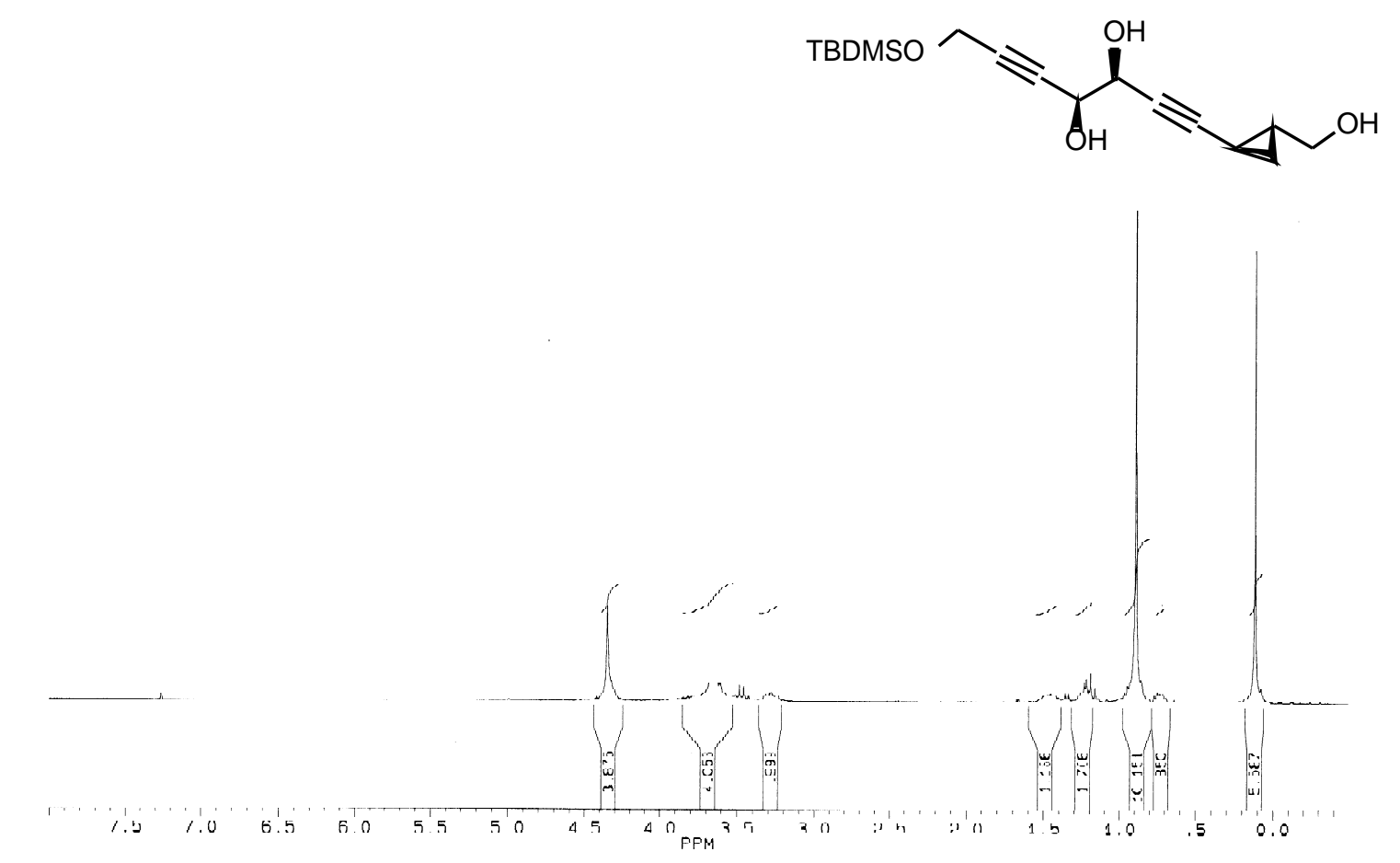

${ }^{1}$ H-NMR-Spektrum von 1-tert-Butyldimethylsiloxy-7-hydroxymethylcyclopropyl-2,6-in-4,5-ol (104)

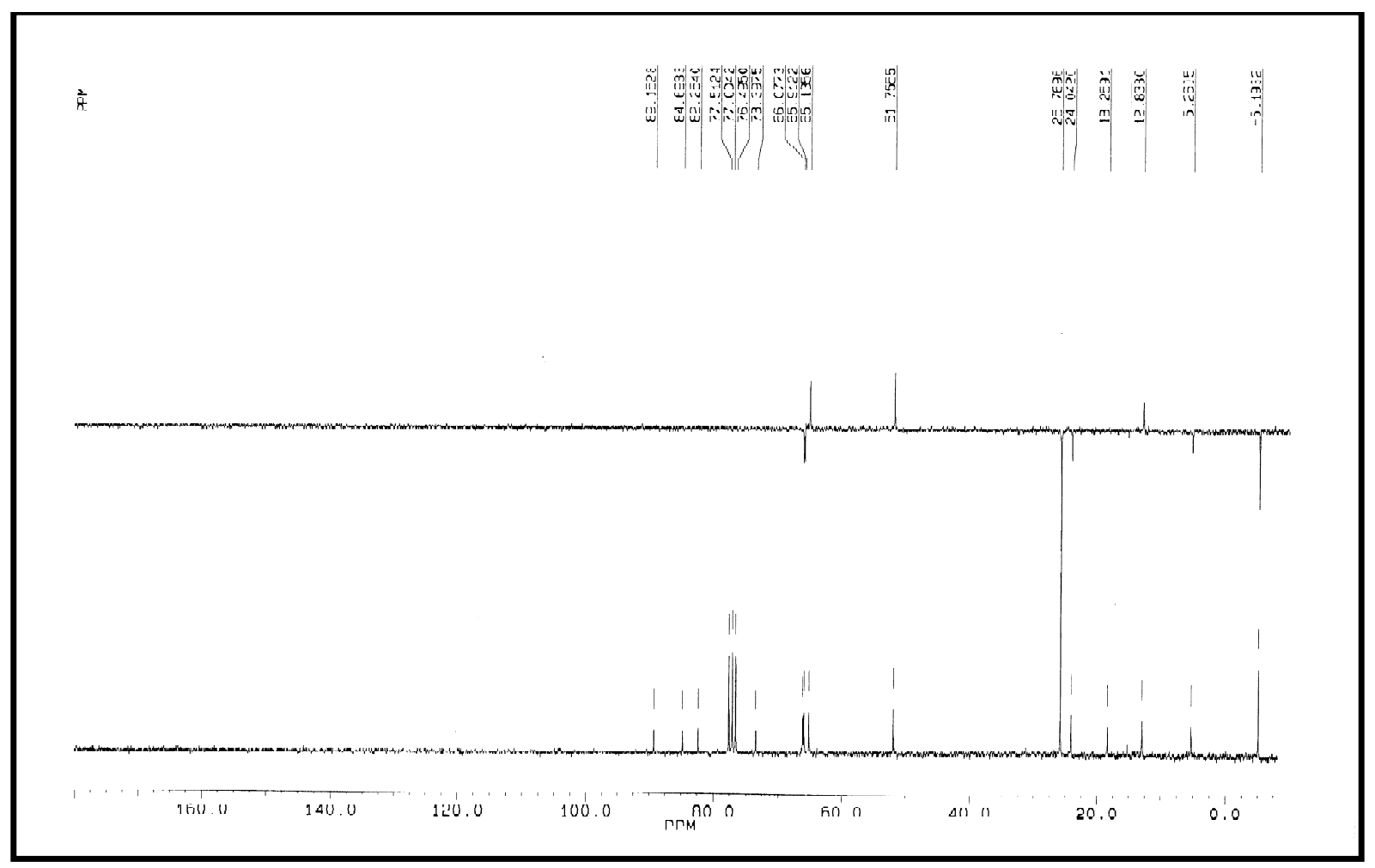

${ }^{13}$ C-NMR-Spektrum von 1-tert-Butyldimethylsiloxy-7-hydroxymethylcyclopropyl-2,6-in-4,5-ol (104) 


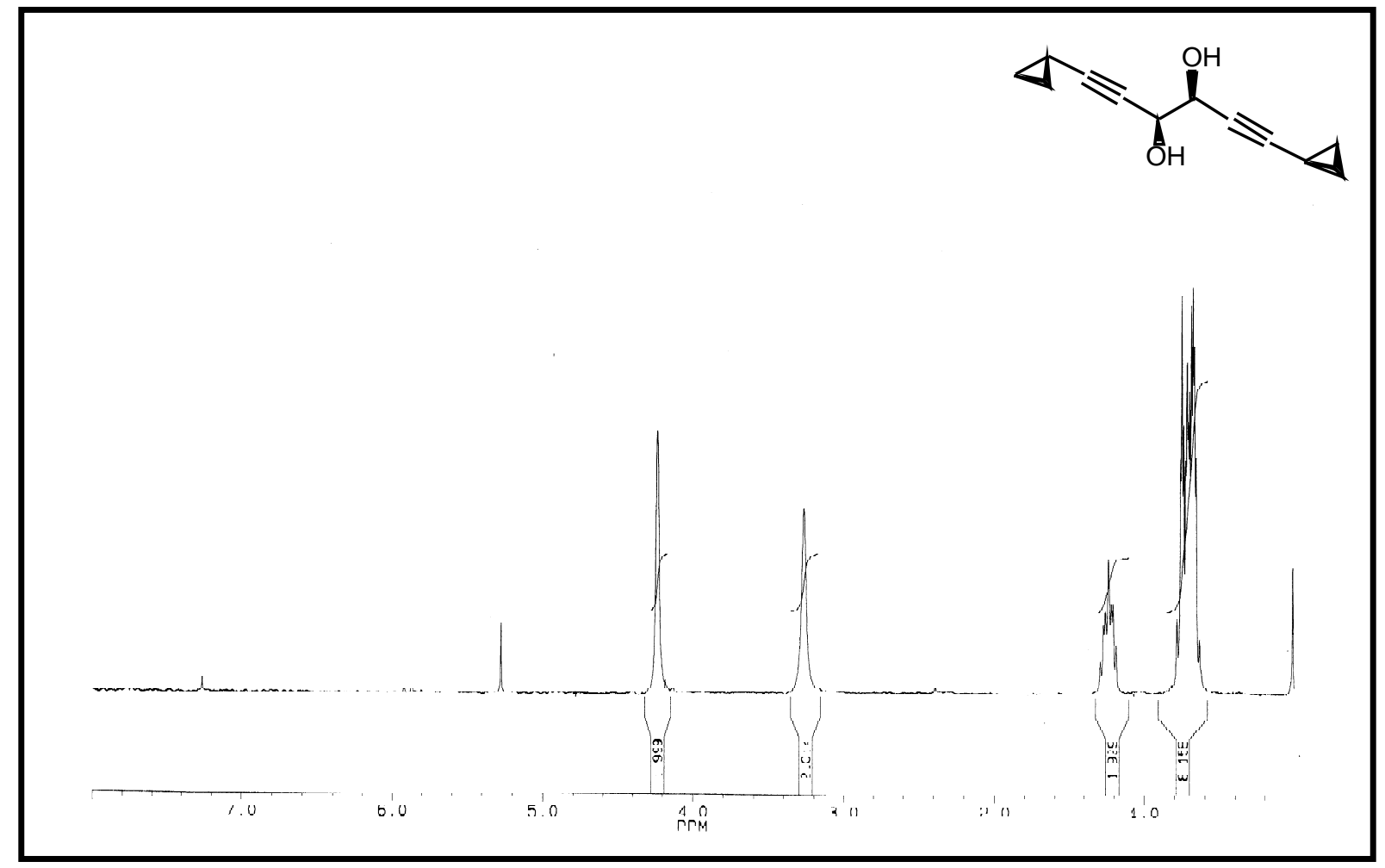

${ }^{1}$ H-NMR-Spektrum von (3R,4R)-1,6-Bis(Cyclopropyl)hex-1,5-diin-2,3-diol (119)

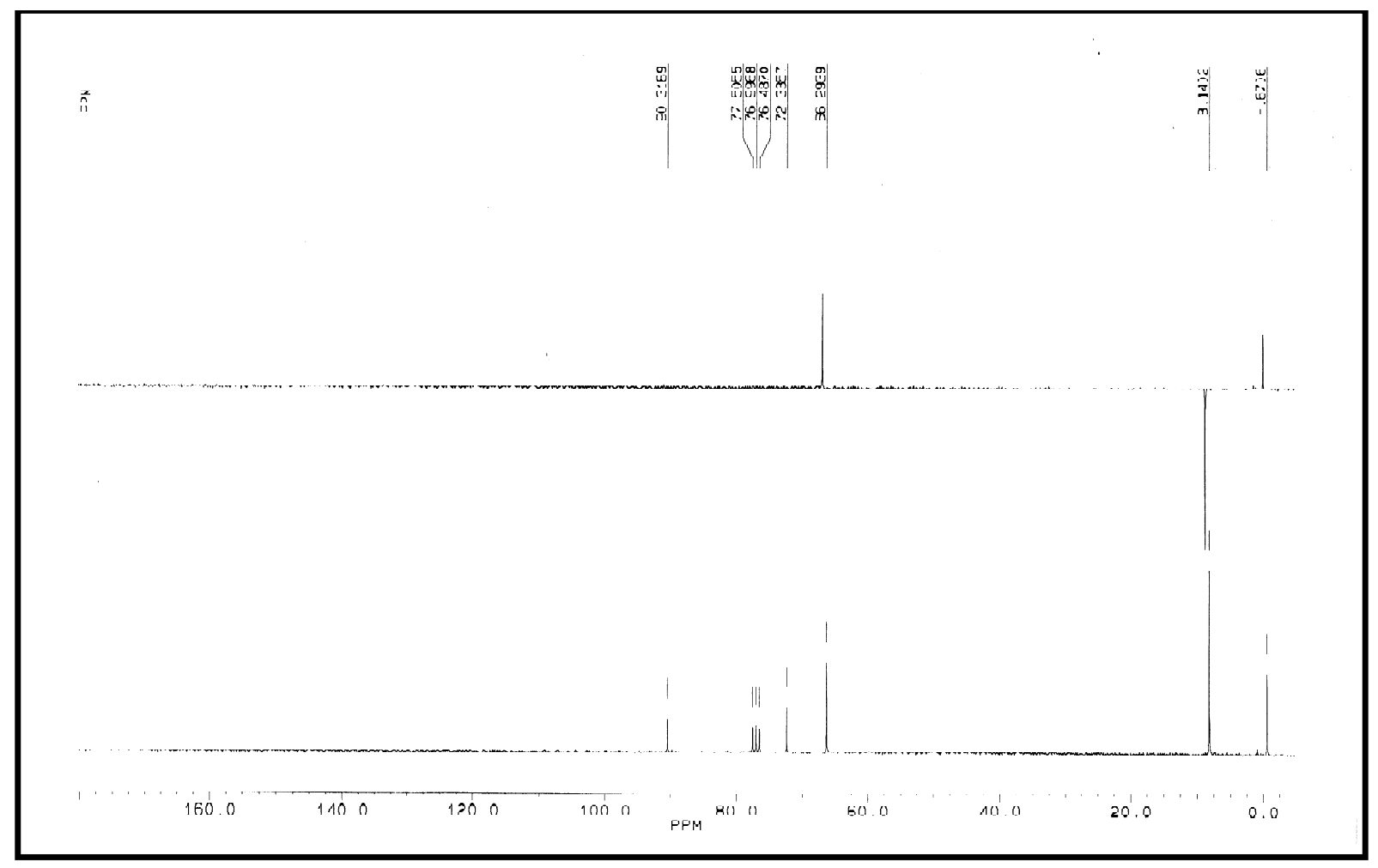

${ }^{13}$ C-NMR-Spektrum von (3R,4R)-1,6-Bis(Cyclopropyl)hex-1,5-diin-2,3-diol (119) 

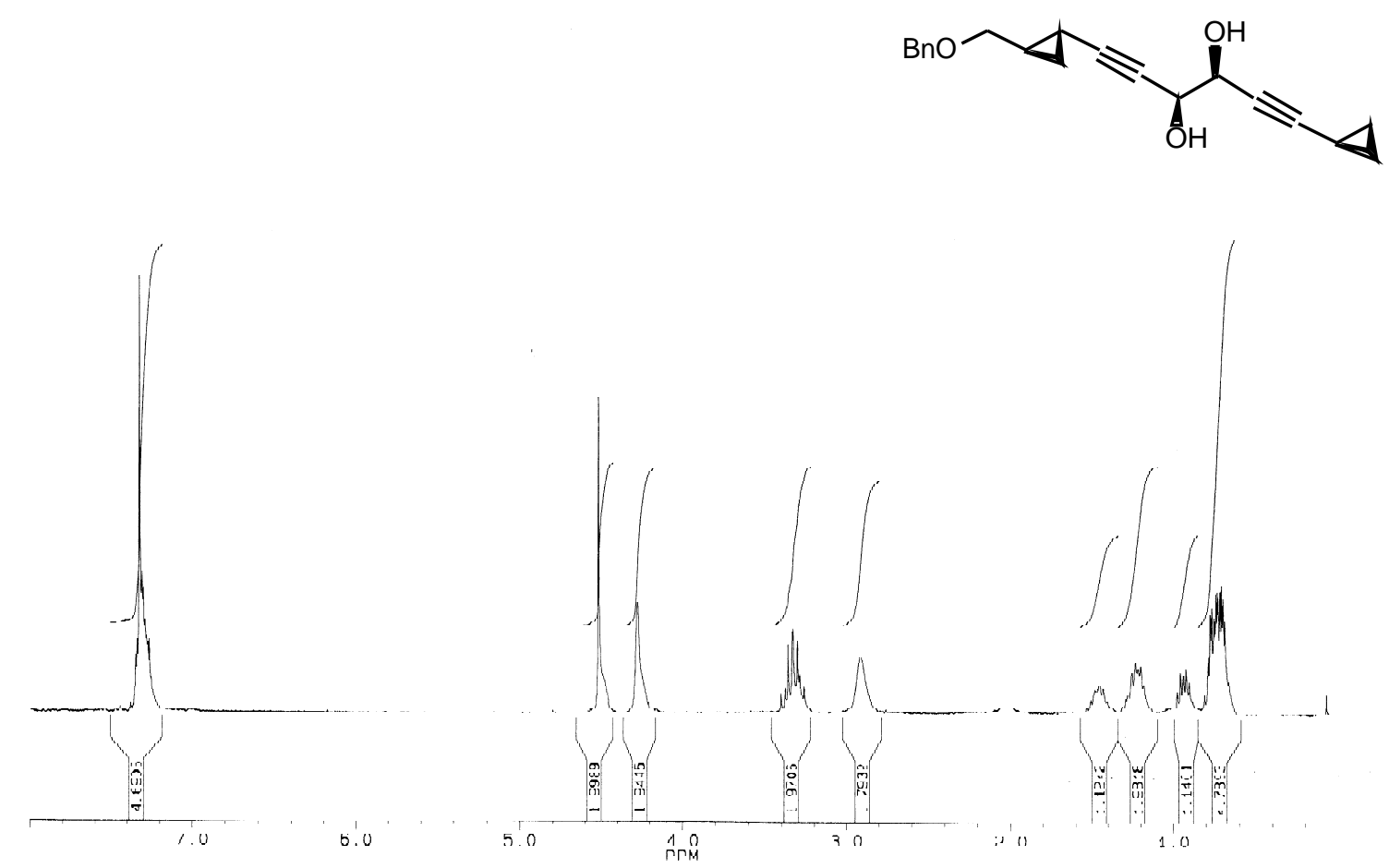

${ }^{1} H$-NMR-Spektrum von (3R,4R)-1-(Benzyloxymethylcyclopropyl)-6-cyclopropylhex-1,5-diin2,3-diol (120)

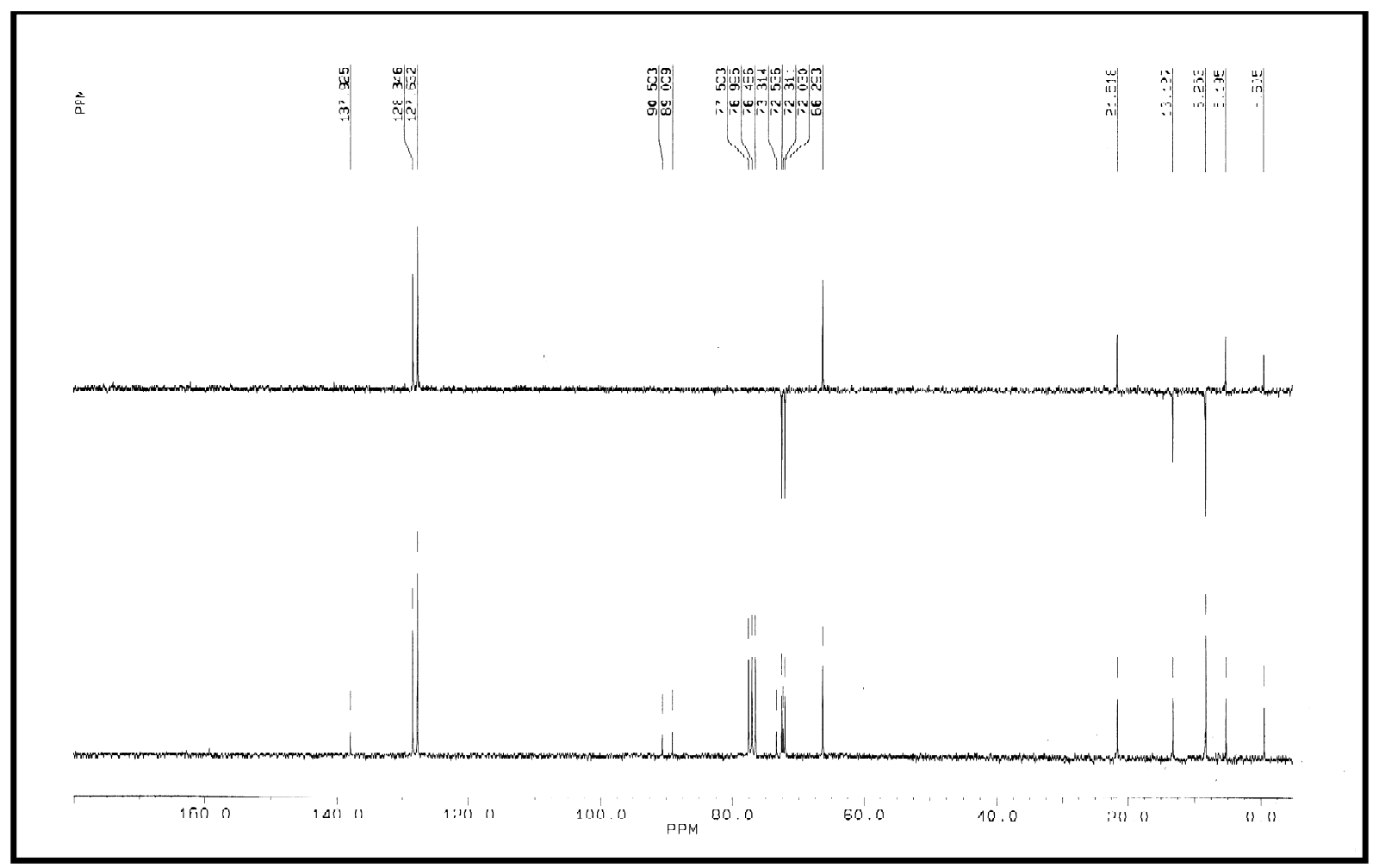

${ }^{13}$ C-NMR-Spektrum von (3R,4R)-1-(Benzyloxymethylcyclopropyl)-6-cyclopropylhex-1,5-diin2,3-diol (120) 


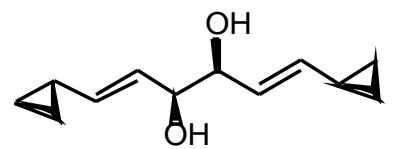

1.0

6.0
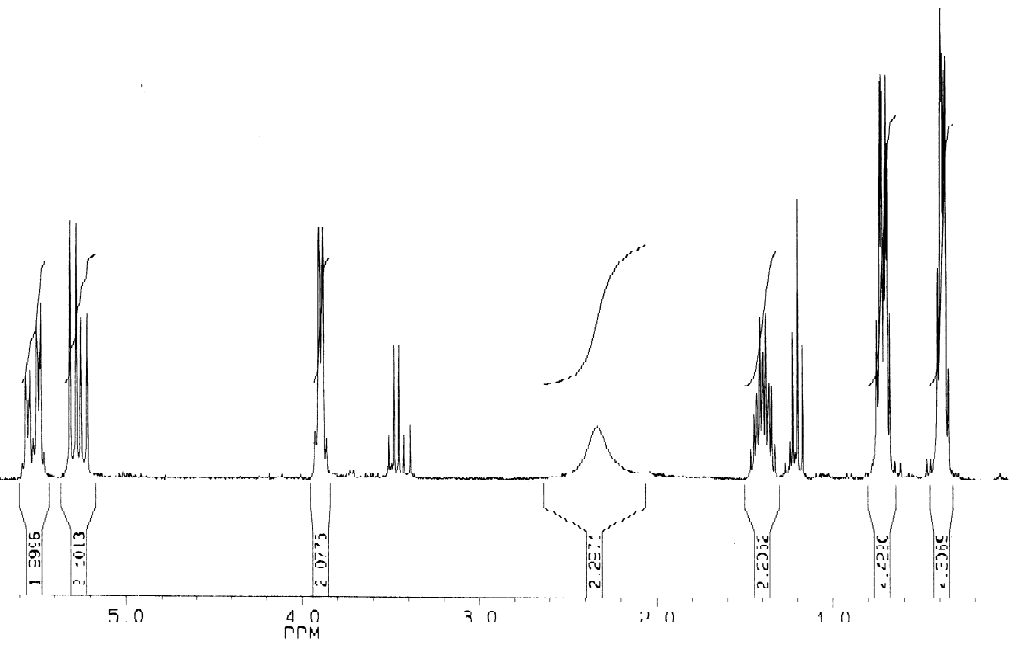

${ }^{1} H$-NMR-Spektrum von (3R,4R)-1,6-Bis(cyclopropyl)hexa-1,5-dien-3,4-diol (147)

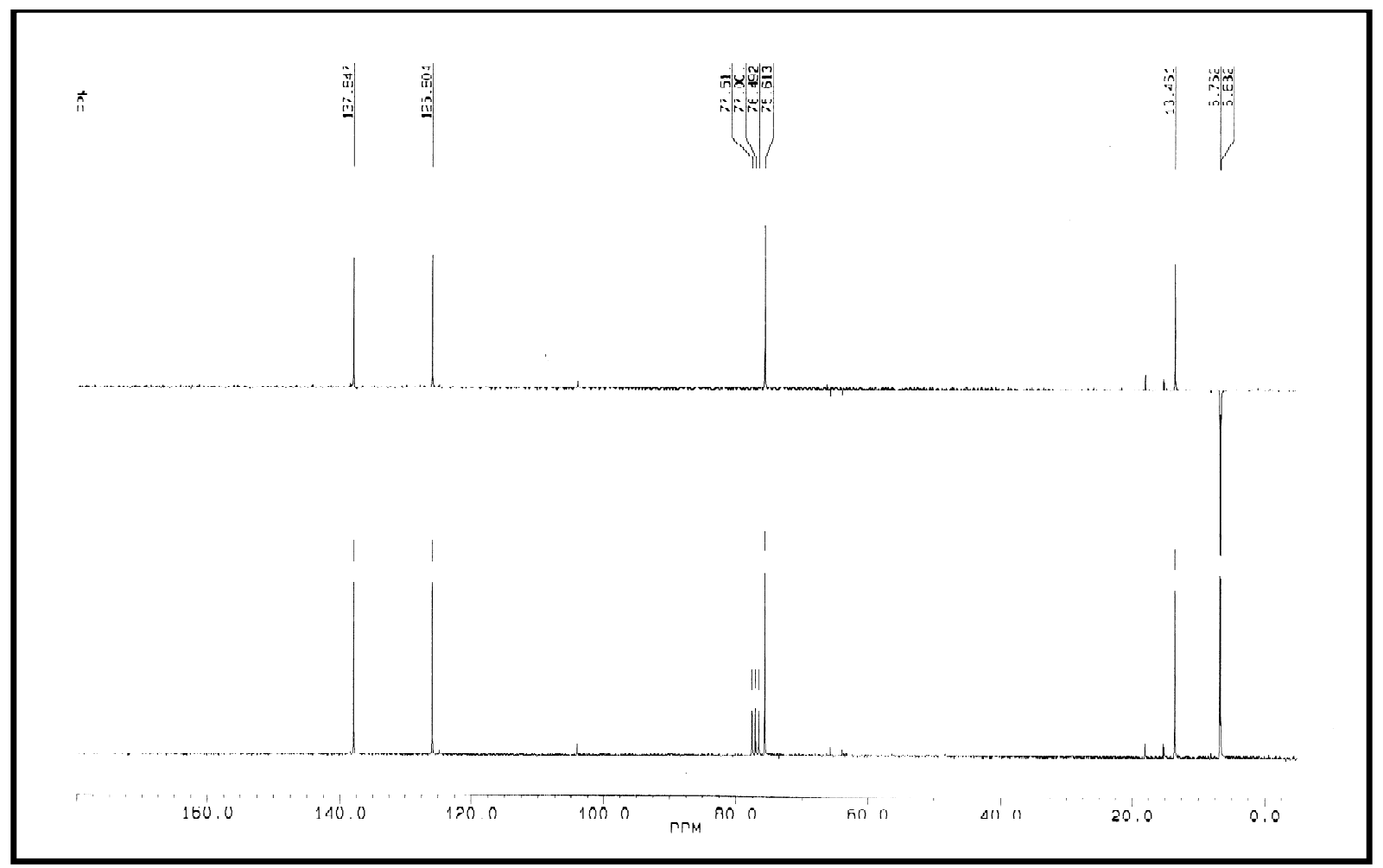

${ }^{13}$ C-NMR-Spektrum von (3R,4R)-1,6-Bis(cyclopropyl)hexa-1,5-dien-3,4-diol (147) 

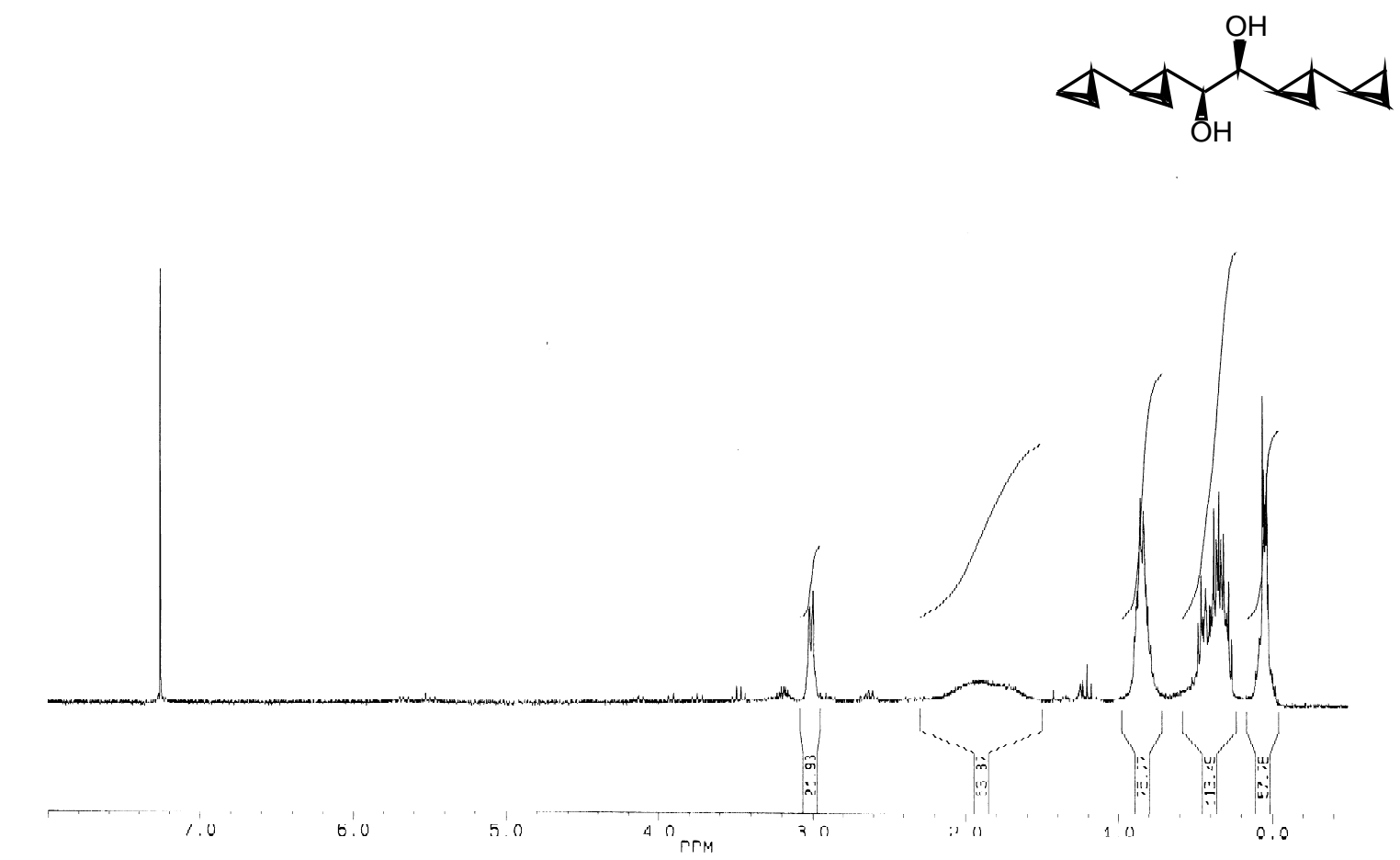

${ }^{1} H$-NMR-Spektrum von $(3 R, 4 R, 5 R, 6 R, 7 R, 8 R)-1,2-B i s($ bicyclopropyl)ethan-1,2-ol (148)

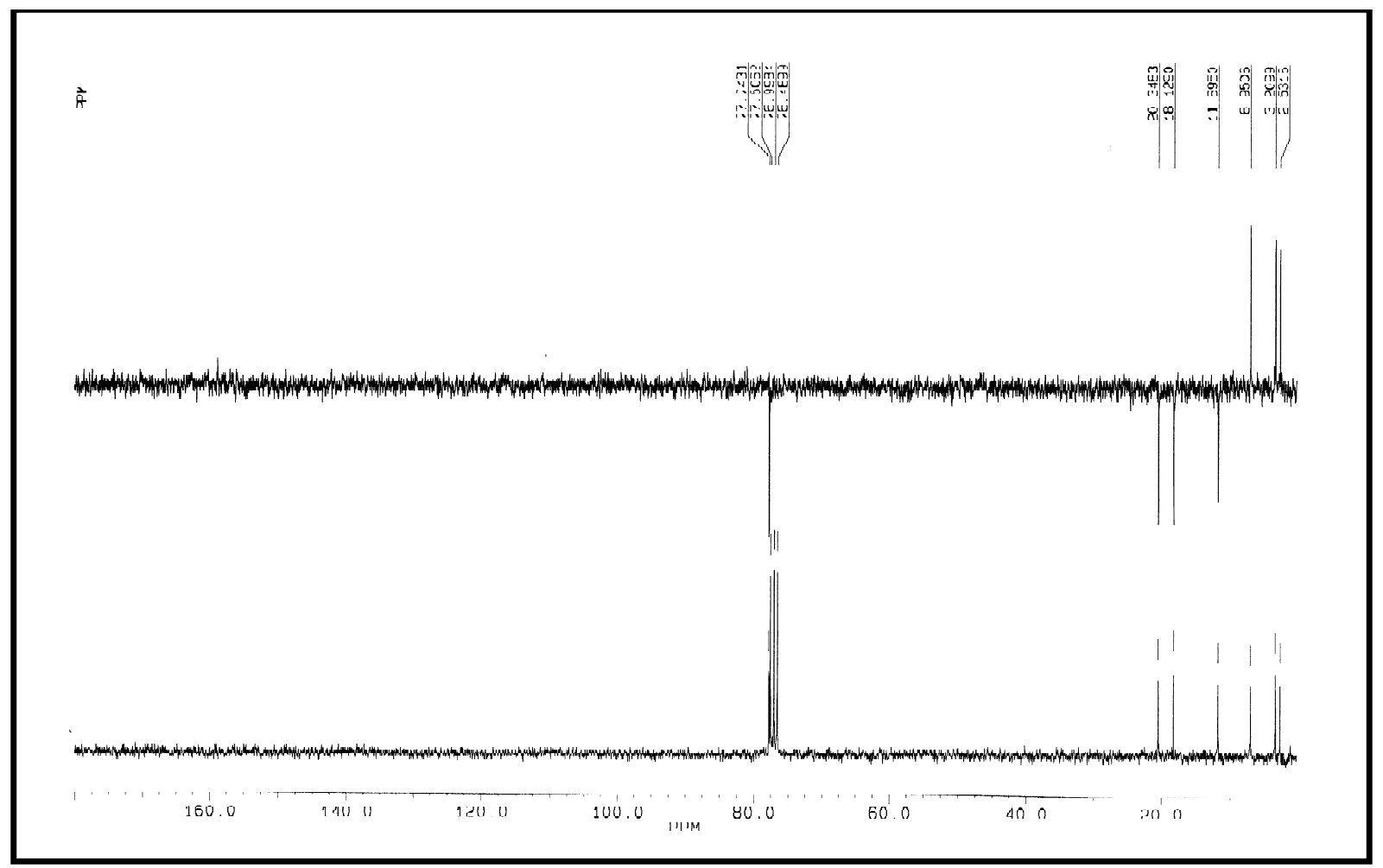

${ }^{13} C$-NMR-Spektrum von $(3 R, 4 R, 5 R, 6 R, 7 R, 8 R)-1,2-B i$ (bicyclopropyl)ethan-1,2-ol (148) 

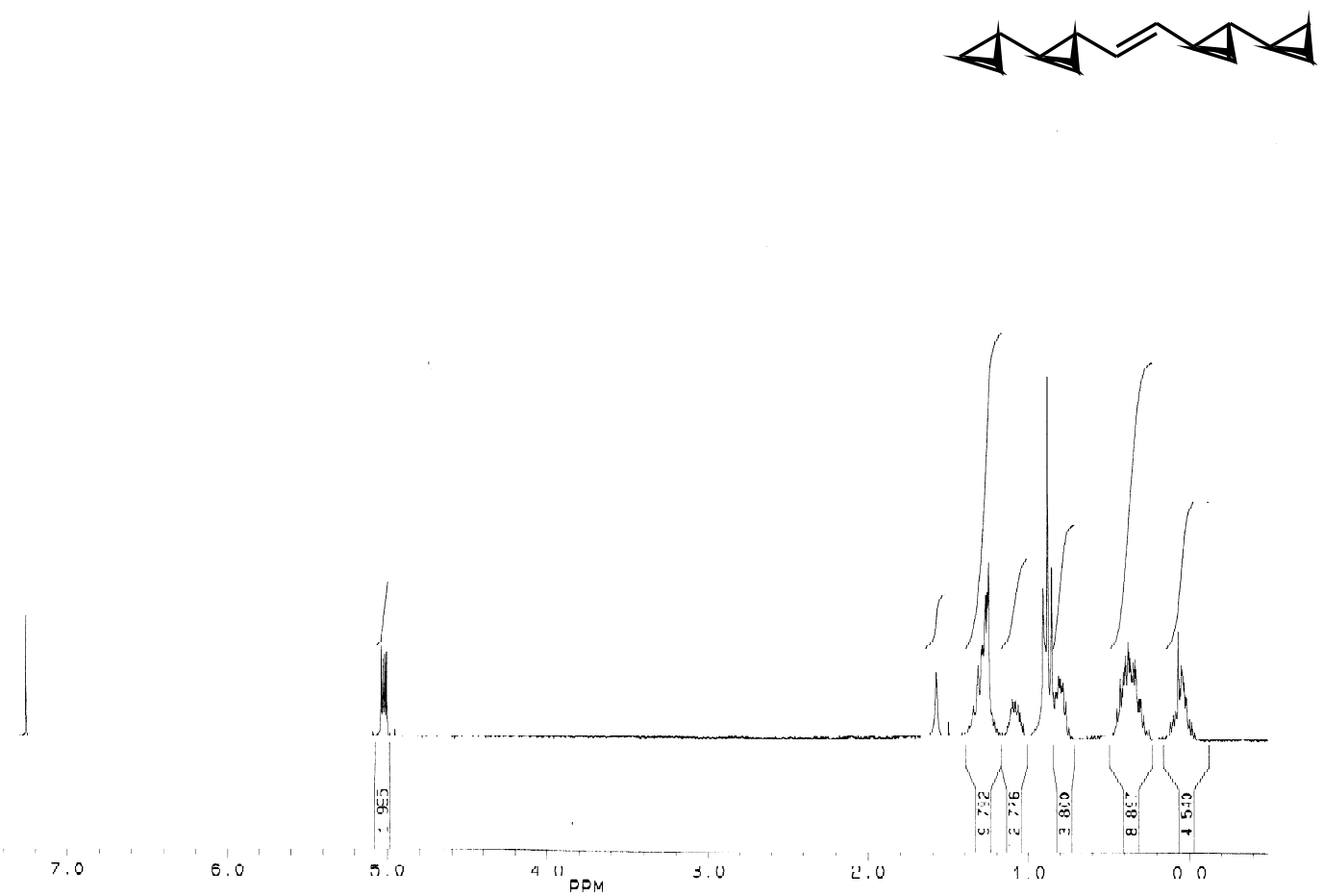

${ }^{1} H$-NMR-Spektrum von $(3 R, 4 R, 5 R, 6 R, 7 R, 8 R)-(E)-1,2-B i$ (bicyclopropyl)-ethen (149)

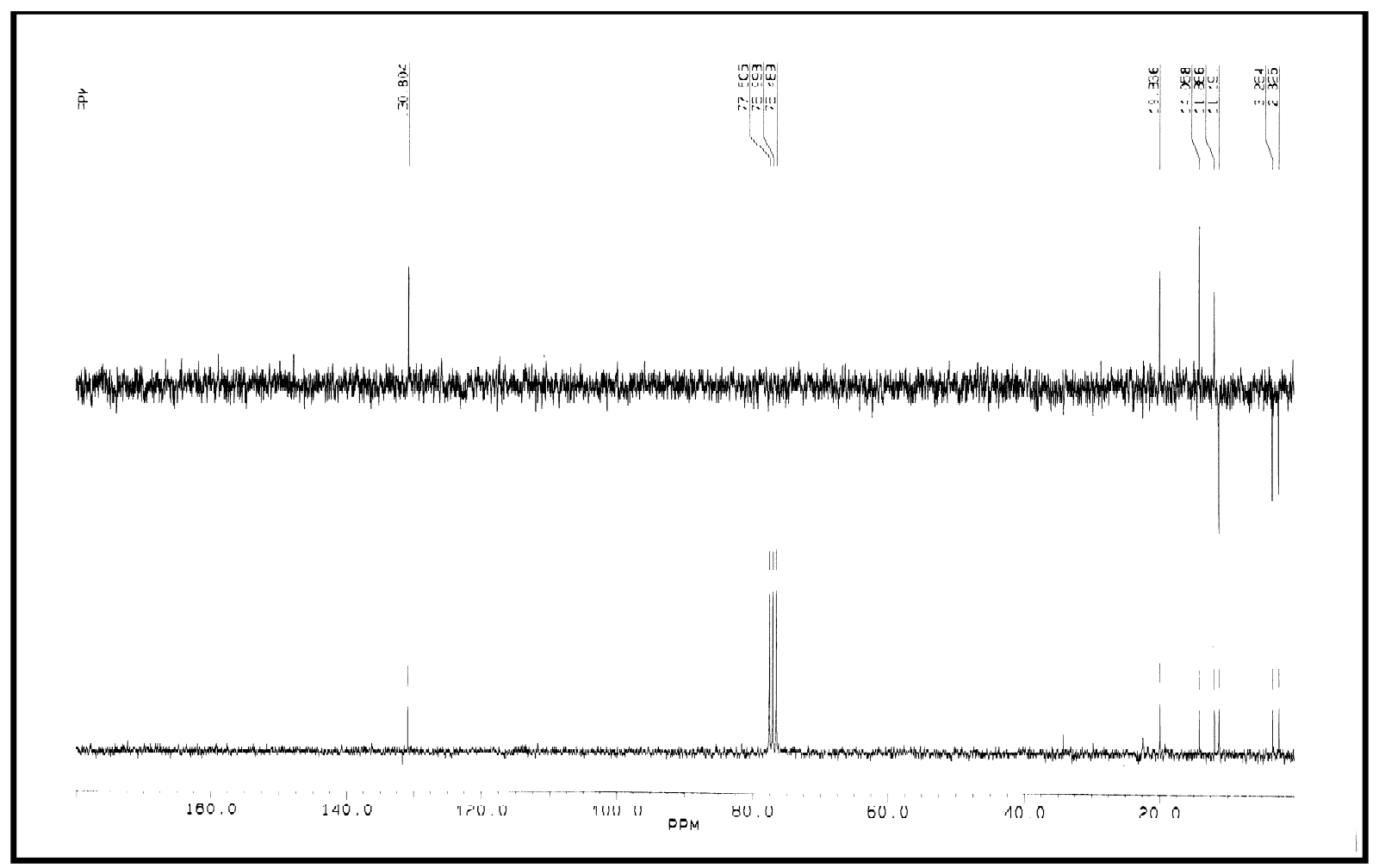

${ }^{13} C$-NMR-Spektrum von $(3 R, 4 R, 5 R, 6 R, 7 R, 8 R)-(E)-1,2-B i s($ bicyclopropyl)-ethen (159) 


\section{Danksagungen}

Für den wissenschaftlichen Unterricht danke ich den Herren Professoren und Dozenten R. Brückner, K.-H. Hoyermann, G. F. Kahl, U. Klingebiel, H. Laatsch, H. Lackner, K. Luther, A. de Meijere, A. Meller, E. Puschmann, H. W. Roesky, E. Schwarzmann, L. F. Tietze, J. Troe, H. Gg. Wagner.

Herrn Dr. Remberg und Frau Udvarnoki danke ich für die Aufnahme der Massenspektren. Herrn F. Hambloch danke ich für die Durchführung der Elementaranalysen.

Ich bedanke mich bei allen Mitarbeitern des Arbeitskreises de Meijere, besonders bei Thomas Perkovic, Markus Kordes, Carsten Ricker, Stephan Müller, Ingo Emme, Hanno Nüske, Markus Nötzel, Sandra Löhr, Markus Tamm, Claudia Thies, Jörg Hellwig, Stefan Beußhausen und Frau Langerfeldt für die Zusammenarbeit und das angenehme Arbeitsklima.

Besonderer Dank gilt auch meinen Eltern, die mich in jedweder Weise unterstützt haben. Am meisten Dank gilt meiner Frau, die mir in allen Lebenslagen zur Seite stand. Vielen Dank an Maximilian, der immer noch lachen konnte, auch wenn mal wieder alles schiefgelaufen war. 


\section{Lebenslauf}

Am 21. Februar 1969 wurde ich als einziges Kind des Möbelpolsterers und Dekorateurs Manfred Wrobel und seiner Ehefrau Ursula Wrobel, geb. Brosig in Verden an der Aller geboren. Seit Februar 1994 bin ich verheiratet und habe ein Kind.

Von 1975 bis 1979 besuchte ich die Nicolai-Grundschule. Danach wechselte ich zur Orientierungsstufe und besuchte danach von 1981 bis 1988 das Domgymnasium in Verden, an dem ich im Juni 1988 die allgemeine Hochschulreife erlangte.

Von Juli 1988 bis Juni 1990 absolvierte ich eine Ausbildung zum Reserveroffizieranwärter beim 4. Flugabwehrregiment 11.

Zum Wintersemester 1990/91 nahm ich das Studium der Chemie an der Georg-August-Universität Göttingen auf. Im Februar 1994 legte ich die Diplomvorprüfung ab. Im Anschluß daran fertigte ich unter der wissenschaftlichen Anleitung von Herrn Prof. A. de Meijere meine Diplomarbeit zu dem Thema "Vorstudien zur Synthese des Antibiotikums FR-900848" an.

Im Mai 1996 wurde mir der akademische Grad "Diplom-Chemiker" zuerkannt. Seitdem arbeite ich im selben Arbeitskreis an meiner Dissertation über das Thema "Cyclopropananaloga mehrfach ungesättigter Fettsäuren“. In diesem Rahmen war ich als wissenschaftlicher Mitarbeiter für den Sonderforschungsbereich 416 angestellt. 